\title{
The diversity of root nodule bacteria associated with Lebeckia species in South Africa
}

by

Francina Lebogang Phalane

Dissertation submitted in partial fulfillment of the requirements for the degree of

Magister Scientiae

in the

Department of Microbiology and Plant Pathology

Faculty of Natural and Agricultural Sciences

University of Pretoria

Pretoria

Promoter: Dr. E.T. Steenkamp

Co-promoter: Dr I.J. Law

May 2008 


\section{TABLE OF CONTENTS}

Page

ACKNOWLEDGEMENTS $\quad 4$

$\begin{array}{ll}\text { PREFACE } & 5\end{array}$

$\begin{array}{lr}\text { CHAPTER } 1 & 7\end{array}$

Literature Review

CHAPTER 2

77

Isolation of rhizobia from Lebeckia species indigenous to South Africa and their nodulation properties on Lebeckia and the promiscuous legumes cowpea and siratro

CHAPTER 3

DNA fingerprinting and 16S rRNA gene analysis of rhizobia associated with Lebeckia species in South Africa

CHAPTER 4

136

Concluding remarks

SUMMARY 
I declare that the thesis/dissertation, which I hereby submit for the degree Masters in Microbiology at the University of Pretoria, is my own work and has not previously been submitted by me for a degree at this or any other tertiary institution.

SIGNATURE....................

DATE........................... 


\section{ACKNOWLEDGEMENTS}

Firstly I would like to thank God for keeping me safe and healthy throughout this study. And I also thank the Almighty Father for making this study possible.

I would like to express my sincere gratitude to the following people for their contributions:

Dr. J. L. Staphorst, my mentor, for his advice and encouragement throughout this study

Dr. E. T. Steenkamp for her advice and guidance

Dr. I. J. Law for his assistance and guidance throughout this study

Dr. W. J. Botha for his assistance and advice with the Bionumerics analysis

Marike Trytsman for providing seeds and Margaret Kieser for assisting with the map

All the students in Dr. E. T. Steenkamp's lab and friends for their help and friendship

Colleagues at ARC-PPRI for giving me their support when the need arose

My family for always encouraging me, for the love, support and patience you have shown me

Prof. B. - E. van Wyk and colleagues in the Botany Dept, University of Johannesburg for their help in the location, collection and identification of Lebeckia plant material

The Departments of Nature Conservation in the Northern Cape, Western Cape and Eastern Cape for permission to collect plant and seed samples in their respective reserves. I would also like to thank Pieter Lund of Bleak House farm, Beaufort West for permission and help in collecting Lebeckia spinescens on his property.

Finally I would like to thank the Department of Science and Technology and Agricultural Research Council for financial assistance during my studies 
Nitrogen-fixing diazotrophic root-nodule bacteria are of immense economic importance because of their symbiosis with leguminous plants. Diazotrophic bacteria infect the host legume root inducing the formation of nodules in which the bacteria (also termed rhizobia) replicate and synthesize the enzyme nitrogenase. This enzyme catalyzes the reduction of atmospheric dinitrogen to ammonia for subsequent use by the plant as a major source of nitrogen. Nitrogen is an essential element required by plants for growth and synthesis of protein and is usually the most limiting element in agricultural production as well as being the most expensive component of fertilizer.

The aim of my study was to determine the diversity and taxonomy of a specific group of root nodule bacteria associated with different species of Lebeckia. The genus Lebeckia Thunb. (Family Leguminosae, subfamily Papilionoideae, tribe Crotalarieae) comprises some 33 plant species. These plants are mainly indigenous to the southern and Western Cape regions of South Africa. They are divided into shrubby trifoliate-leaf species in the sections Calobota, Stiza and Viborgioides and suffrutescent unifoliate needle-leaf species in the section Lebeckia. Plants of this genus are adapted to harsh environmental conditions such as are found in the Karoo and Namaqualand. Several Lebeckia species are beneficial, such as $L$. spinescens and L. multiflora, which are valuable as pasture legumes and well grazed by animals especially in winter. All the species have ecological value because of their nitrogen-fixing symbiosis with rhizobia. To my knowledge, no attempts have been made in the past to investigate these microsymbionts of Lebeckia.

Root nodules were collected from Lebeckia species at a wide variety of localities in the western and southern Cape regions of South Africa. Indigenous rhizobia isolated from these nodules were investigated for their nodulation abilities on their respective host plants as well as on non-host promiscuous legumes, cowpea and siratro. The isolates were then characterized using random amplified DNA fingerprinting followed by DNA sequencing of selected isolates. Results presented in this study showed that the indigenous South African genus Lebeckia is nodulated by diverse rhizobia from both $\alpha$ - and $\beta$-Proteobacteria.

The first chapter contains a literature review of symbiotic nitrogen fixation that includes a general description of the biology, inoculant technology and the taxonomy of legumes and 
their rhizobia. The genera within the tribe Crotalarieae (such as Crotalaria, Lotononis, and Aspalathus) were discussed with special reference to the genus Lebeckia. Technical methods used for the classification of rhizobia were also reviewed. Non-DNA-based methods such as host specificity, substrate utilization, antibiotic resistance, morphological characters and biochemical properties as well as DNA based fingerprinting methods (ARDRA, RFLP RAPD, and AFLP), DNA sequence information, analysis of whole genomes, DNA-DNA hybridization and polyphasic approaches were outlined.

The second chapter describes the isolation of 79 rhizobial isolates from the root nodules of 10 Lebeckia species. The isolates were purified and tested for nodulation and nitrogen fixation on cowpea and siratro as well as their host plants. All the isolates fixed nitrogen on their respective Lebeckia hosts, whereas $56 \%$ of the strains were effective on cowpea and $77 \%$ on siratro.

The third chapter describes initial comparison and screening of the isolates by SP-PCR fingerprinting analysis. DNA profiles showed that most of the isolates grouped according to host plant species rather than geographical location. Isolates selected from different clusters were subjected to partial 16S rDNA gene sequencing to confirm their taxonomic identity. This revealed that Lebeckia is nodulated by diverse genera of root nodule bacteria from both the $\alpha$-Proteobacteria (Bradyrhizobium, Mesorhizobium, and Sinorhizobium) as well as the $\beta$-Proteobacteria (Burkholderia). The final chapter (Chapter 4) provides concluding remarks on my results and possible future avenues of research on the Lebeckia rhizobia. 


\section{CHAPTER 1}

\section{LITERATURE REVIEW}

\section{TABLE OF CONTENTS}

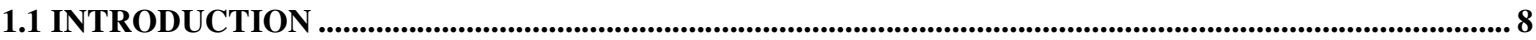

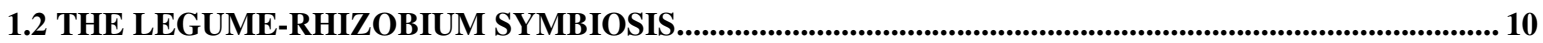

1.3 TAXONOMY AND EVOLUTION OF LEGUMES...................................................................................... 12

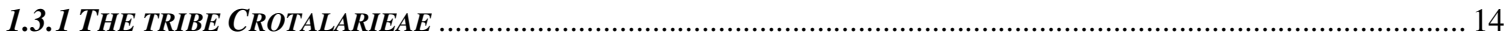

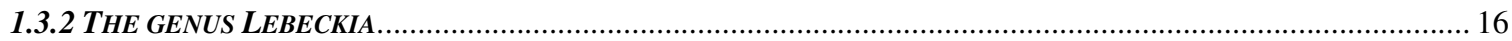

1.4 THE TAXONOMY OF RHIZOBIA ........................................................................................................... 17

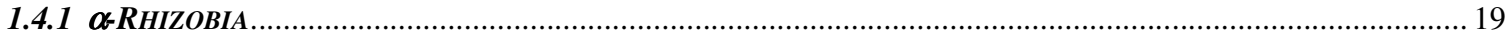

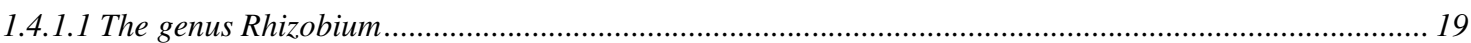

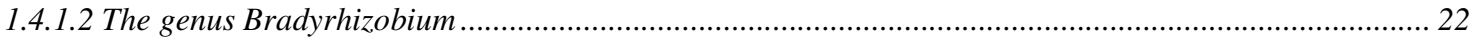

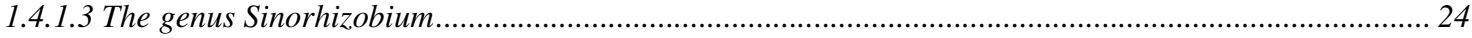

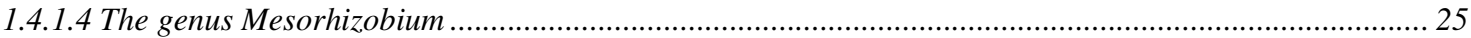

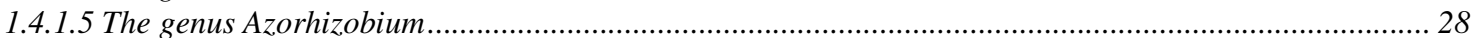

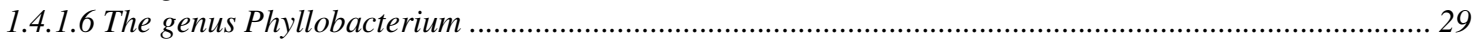

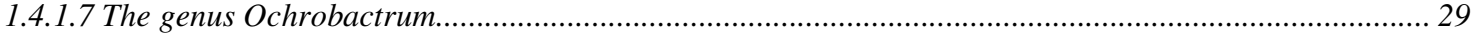

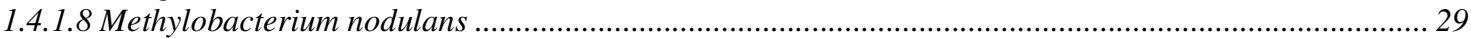

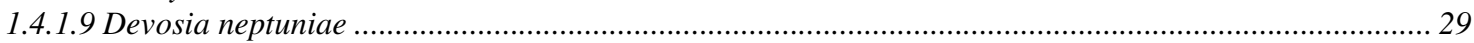

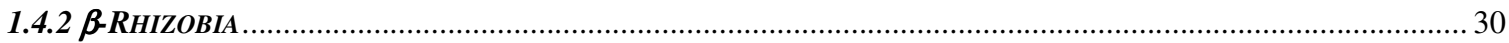

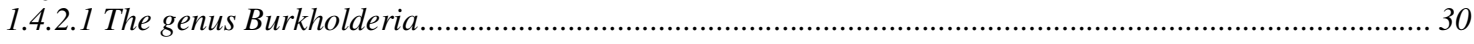

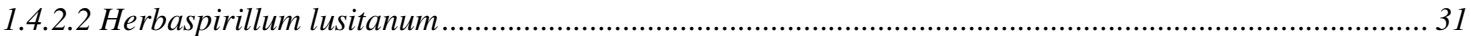

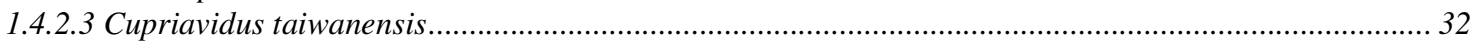

1.5 METHODS FOR CLASSIFYING RHIZOBIA ................................................................................................ 32

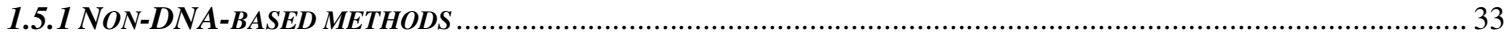

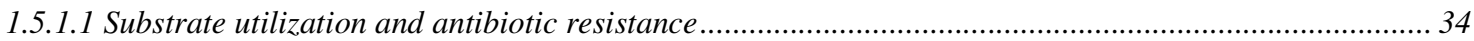

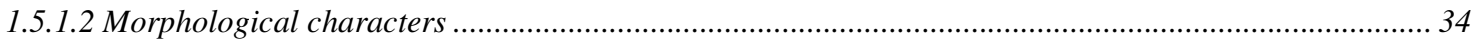

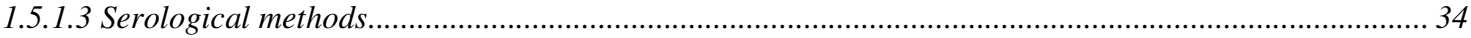

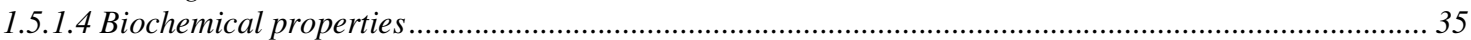

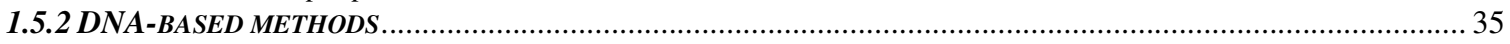

1.5.2.1 Analyses of whole genomes using fingerprinting methods.................................................................. 37

1.5.2.2 Analyses of whole genomes using non-fingerprinting methods ........................................................... 38

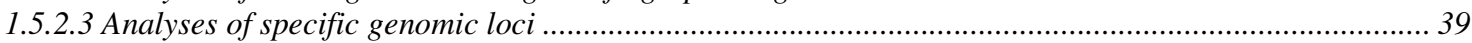

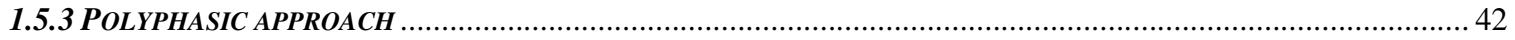

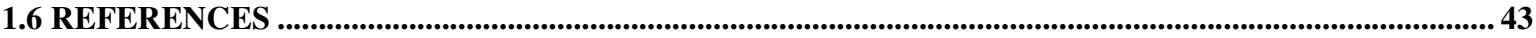

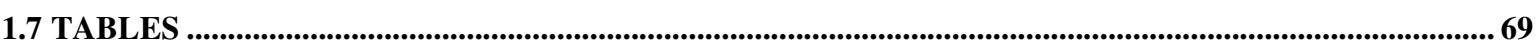

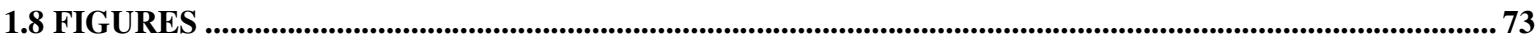




\subsection{INTRODUCTION}

Nitrogen in the form of protein represents a significant part of human and animal diets. In 1994, individual protein consumption of the 5.3 billion people on earth averaged about $70 \mathrm{~g}$ of protein/day or 23 million tonnes of N/annum (Vance \& Graham, 1995). Maintenance of this is estimated to require a doubling or tripling of crop production together with the use of large areas now considered marginal, as the earth's population doubles over the next 40 years (Vance \& Graham, 1995). In terms of importance for plant growth and development, nitrogen acquisition and assimilation is second only to photosynthesis (Granli \& Bøckman, 1994). Yet nitrogen is perhaps the single most important factor currently limiting plant yields (Graham \& Vance, 2003) and is largely alleviated by application of commercial fertilizer. In 1994, world fertilizer application was estimated at 77 million tonnes/year (Granli \& Bøckman, 1994).

Agricultural application of nitrogen fertilizer has great disadvantages as it results in the leaching of toxic nitrate into the soil and groundwater (Hardy \& Eaglesham, 1995). These and other nitrogen-based toxic chemicals threaten human health because they may cause illnesses such as cancer (Bohlool et al., 1992; Graham \& Vance, 2003). From an environmental perspective, contamination with inorganic and organic nitrogen compounds can lead to eutrophication of surface water, while reactive gaseous nitrogen oxides are associated with ozone layer depletion (Bohlool et al., 1992; Graham \& Vance, 2003). Industrial nitrogen fertilizer production is also very expensive, not only in terms of money, but also in terms of requirements for non-renewable energy resources (Hardy \& Eaglesham, 1995). To synthesize ammonia from nitrogen under high temperature and pressure conditions during the Haber-Bosch process involves extremely large inputs of fossil fuel energy. It is estimated that the cost of nitrogen fertilizer production is around \$20-60 billion per year (Hardy \& Eaglesham, 1995).

In contrast, biological nitrogen fixation (BNF) by so-called diazotrophic bacteria is sustainable, less polluting and significantly cheaper compared to industrial nitrogen fertilizer (Vance, 2001; Giller, 2001; Graham \& Vance, 2003). Diazotrophs are known from most lineages of the Eubacteria (i.e. proteobacteria, cyanobacteria, actinobacteria spirochaetes, clostridiales and chlorobiales) and the Archaeal methanogens (Doyle \& Luckow, 2003; Kneip et al., 2007). They may be free-living in soil and water or form associations and symbioses with certain animals, protists, fungi and a variety of plants (e.g. Kneip et al., 2007). It is in these associations, especially with plants, where diazotrophs contribute most to BNF and agriculture (Giller, 2001; 
Graham \& Vance, 2003). BNF accounts for about $65 \%$ of agricultural nitrogen (roughly 140 million metric tonnes/year) and is becoming increasingly important in crop productivity especially for sustainable systems, small scale operations and marginal land use (Granli \& Bøckman, 1994; Hardy \& Eaglesham, 1995).

Overall, the symbiosis between the so-called rhizobial Proteobacteria and leguminous plants is responsible for by far the greatest amount of BNF in agriculture and plays a central role in sustainable agricultural systems (Giller, 2001; Graham \& Vance, 2003). As a result, grain and forage legumes account for $27 \%$ of the world's primary crop production with grain legumes alone contributing 33\% of the dietary protein needs of humans (Graham \& Vance, 2003). In South Africa, Strijdom and Wassermann (1984) estimated that 100000 tonnes of nitrogen are fixed per year - a figure that has probably doubled or tripled in the present decade. They also estimated a potential for the addition of 12.5 million ha of pasture leading to a total of 16 million ha which could fix an estimated 1.4 million tonnes of nitrogen (Strijdom \& Wassermann 1984). This could enrich the soil by more than 400000 tonnes of nitrogen, an amount comparable to the total nitrogen fertilizer sold annually in the country (Strijdom, 1998).

Developing countries are faced with the dilemma of increasing food production due to escalating population numbers. The problem is compounded by the low nutrient status of many agricultural soils and the expense and often limited availability of nitrogen and other fertilizers. BNF is a comparatively cheap alternative that can contribute directly to a growing crop and can increase soil fertility. Technologies based on the use of nitrogen-fixing plants are ideal for use by farmers in developing countries who do not have the financial resources to buy fertilizers even when these are available. It is clear that BNF, with its ability to capture an inexhaustible resource must play a fundamental role in agriculture (Giller, 2001). For optimal exploitation of this process, knowledge of legumes and their microsymbiont partners is essential.

The aim of this literature study is to review literature pertaining to the legume-rhizobium symbiosis and the taxonomy of rhizobia, as well as to provide a detailed overview of methods used to classify rhizobia. The taxonomy of legumes, especially those in the tribe Crotalarieae and genus Lebeckia, is also emphasized. 


\subsection{THE LEGUME-RHIZOBIUM SYMBIOSIS}

An important step in the legume-rhizobium symbiosis is the development of nodules. Nodules are lateral outgrowths of the roots (e.g peanut) and stems of legumes as a result of an infection of the root-hair (e.g soybean) or cracks in the roots by rhizobia. Rhizobia enter their host's roots through the root hair. The infection is preceded by deformationor curling of root hairs and the formation of an infection thread caused by the rhizobial strains (Somasegaran \& Hoben 1994). Effective nodules can have various forms ranging from spherical to branched and globular, but all with a pink leghaemoglobin centre, while the ineffective nodules have white centres (Corby et al., 1983; Heichel \& Vance, 1983; Sprent 2001). Once the bacteria penetrate the root hair, bacteroids differentiate in the nodules and synthesize the rhizobial nitrogenase which fixes atmospheric nitrogen for the plant (Somasegaren \& Hoben, 1994; Dean et al., 1993).

Legumes are the only plant family, with the notable exception of Parasponia (family Ulmaceae) capable of being nodulated by rhizobia in the bacterial family Rhizobiaceae (Rhizobium, Sinorhizobium, Azorhizobium, Bradyrhizobium) and those in the Beta-Proteobacteria genera Burkholderia, Herbaspirillum and Cupriavidis, as well as the methylotrophic Methylobacterium (Sawada et al., 2003; Weir et al., 2004; Wolde-Meskel et al., 2005; Weir, 2006). The mechanism by which host and symbiont recognize one another involves an interchange of molecular signals during infection and nodule formation (Hungria \& Stacey, 1997). Bacterial nodulation gene products stimulate the expression of plant genes that result in the formation of nodules. The nitrogen-fixation (nif, fix) and nodulation genes are located on the sym loci which may be encoded on plasmids in the fast-growing rhizobia or chromosomal islands in the slow-growing rhizobia. The nodulation genes control the formation and structure of Nod factors (NFs) that are the primary signalling molecules responsible for host infection and nodule formation (van Rhijn \& Vanderleyden, 1995). The NF consists of a lipo-chitooligosaccharide molecule which is formed by the products of the structural nodulation genes (nodABC).

During the symbiosis, the main energy source for nitrogen-fixation is provided by the legume in the form of photosynthetically fixed carbon (Minchin et al., 1986). The latter is then translocated into the nodules in the form of sucrose, which is used as reductant for the conversion of atmospheric nitrogen to ammonia by the bacterial nitrogenase. Numerous estimates have been made on the carbon cost of symbiotic nitrogen-fixation, most of which are in the range between 5 and $18 \mathrm{~g}$ of carbon per $\mathrm{g}$ of nitrogen fixed. In addition to driving the 
nitrogenase reaction in bacteroids, up to $33 \%$ of total plant photosynthate may be required when nodule development and maintenance and transport costs are considered (Minchin et al., 1986). The bulk of the ammonia is however transferred to the legume partner to meet its nutritional nitrogen needs for synthesizing essential compounds such as protein, nucleic acids and chlorophyll (Minchin et al., 1986).

Within the legume family, there is a great deal of host specificity. For example, lucerne is only nodulated by Rhizobium meliloti whereas soybean is mainly nodulated by Bradyrhizobium japonicum. The overall structure of the NF is believed to influence the host range of a specific rhizobial strain (Carlson et al., 1993; Van Rhijn \& Vanderleyden, 1995; Lopez-Lara et al., 1996; Spaink et al., 1998; Perret et al., 2000; Moulin et al., 2004;). The final structure of the NF core is determined by a class of nodulation genes often referred to as host-specific nodulation ( $h s n$ ). The products of these genes catalyze the modification of the NF core by for example, transferring fatty-acid and other moieties to it. Rhizobial species differ broadly in terms of the activity of and complement of hsn genes (Carlson et al., 1993; Van Rhijn \& Vanderleyden, 1995; Lopez-Lara et al., 1996; Moulin et al., 2004;).

The practical consequence of highly specific legume-rhizobium associations for agriculture is that legume crops must be inoculated, especially when planted in soils that do not contain native populations of infective rhizobia. Considerable genetic variation exists within each symbiotic partner and careful selection of an inoculant strain is essential to ensure maximum nitrogenfixing effectiveness with the desired cultivar. Of course, this is more important for a crop such as soybean which is highly selective in its rhizobial requirements, than a less selective crop such as cowpea (Brockwell et al., 1995). Commercial inoculants for legume crops usually consist of rhizobia in a peat carrier and quality control must ensure that adequate numbers of viable rhizobia are applied at planting. Inoculants are generally attached to seed with a sticker and care must be taken to avoid contact with seed-applied pesticides that are often toxic to the rhizobia. An alternative application of inoculant in water suspension is more effective especially when seeds are easily damaged (Brockwell et al., 1995).

An essential consideration in the establishment of nitrogen-fixing systems is response to adversities of soil environment. Since plant productivity can be defined by a single limiting factor in the system, no amount of biologically or chemically fixed nitrogen will increase production if some other factor is limiting growth. Major considerations affecting efficiency of 
the legume symbiosis include soil acidity and related aluminum and manganese toxicity, calcium and phosphorous deficiency, salinity, flooding and the amount of available nitrogen mineralized from organic sources (Bohlool et al., 1992). In addition to reducing the amount of nitrogen derived from BNF, nitrate in soil is inhibitory to nodulation and nitrogen-fixation, thus high level applications of fertilizer nitrogen must be avoided. A frequent and major obstacle to the successful use of legume inoculants is competition from native soil rhizobia for nodulation. Under such circumstances, magnitude of the nitrogen-fixing response to inoculation is related to population indices of soil rhizobia as well as the nitrogen status of the soil (Thies et al., 1991).

\subsection{TAXONOMY AND EVOLUTION OF LEGUMES}

Together with the families Polygalaceae, Surianaceae and Quillajaceae, legumes (family Leguminosae or Fabaceae) form part of the monophyletic order Fabales in the Eurosid Clade I of the rosid angiosperms (Soltis et al., 2000; Angiosperm Phylogeny Group, 2003). Based on DNA-based analyses, members of the Fabaceae are more closely related to one another than to other angiosperms (Doyle et al., 2000; Kajita et al., 2001). According to Doyle et al. (2000), the monophyletic nature of this taxon is also supported by non-molecular traits such as petal imbrication where the abaxial pair are internal to the lateral petals in the bud; a single carpel in the flower; endothecium ribs less than six per cell; and rib spacing less than twice the width.

The Fabaceae comprise some 18000-19000 species and about 600-750 genera (Allen \& Allen, 1981, Van Wyk, 1991; Sprent, 1995; Chen et al., 2003; Graham \& Vance, 2003; Wojciechowski, 2003; Weir et al., 2004; Wolde-Meskel et al., 2005), although estimates of actual numbers vary considerably with different authors. Nevertheless, the family Fabaceae is the third largest family of flowering plants in the world (Corby et al., 1983; Sprent, 1995; Doyle \& Luckow, 2003) and includes herbs, trees and shrubs. They are widely distributed and adapted to a variety of environments, although herbaceous species and shrubs are mostly found in cool temperate climates while leguminous trees are abundant in subtropical/tropical regions (Van Wyk, 1991). Overall, the Fabaceae appears to be most diverse in seasonally dry tropical forests and temperate arid to semi-arid shrub lands, while they are relatively poorly represented in mesic temperate regions such as arctic and alpine areas, as well as under stories of cool temperate forests (Wojciechowski, 2003). This distribution is thought to be linked to the nitrogen-demanding metabolism associated with semi-arid and arid habitats, conditions in which the Fabaceae are able to produce leaves more efficiently than most other plant families (McKey, 1994). 
In the floral fossil record, legumes are not represented among the rich mid-Cretaceous about 90 million years ago (mya) and remained relatively under-represented until about 54-35 mya ago (Taylor, 1990; Herendeen et al., 1992). The results of DNA-based analyses suggest that legumes evolved around 60 mya early in the Tertiary period (Lavin et al., 2005; Cronk et al., 2006; Sprent, 2007). This contrasts with previous ideas that legumes had a Gondwanan origin during the late Cretaceous (65-145 mya) in Africa, from where they spread to the rest of the world (Corby et al., 1983). Contemporary hypotheses for explaining the global distribution of legumes propose a northern hemisphere origin and migration to the rest of the world across land bridges and via weather related phenomena (Doyle \& Luckow, 2003; Cronk et al., 2006; Sprent, 2007).

Nodulation is currently speculated to have arisen approximately 58 mya (Sprent, 2007; Sprent \& James, 2007). The process is thought to have been prompted by an abrupt $5-10^{\circ} \mathrm{C}$ rise in temperature over a range of latitudes and increased atmospheric levels of methane, carbon dioxide and humidity. As a result, carbon cycling through terrestrial ecosystems was greatly enhanced leading to the appearance of a vast plant biodiversity during this period. Sprent (2007) suggests that the probable limitation of nitrogen for plant growth during this period triggered the development of nodulation. Consistent with this idea, the major groups of nodulating legumes also emerged during this time (Sprent, 2007; Sprent \& James, 2007).

The family Fabaceae contains many the economically valuable crops. For example, numerous species are cultivated for food (e.g. beans, peas, peanuts, soybeans and lentils) and forage (e.g., clover or Trifolium spp. and Medicago spp. such as lucerne, while others are favoured as garden ornamental trees and shrubs (e.g. Lebeckia sericea, Acacia spp., Mimosa spp.). Legumes such as clover, soybean, lupin and alfalfa are also commonly used as green manure (Doyle, 1998; Graham \& Vance, 2003; Doyle \& Luckow, 2003). Some members of the family have medicinal properties such as soybeans that produce isoflavones that can reduce the risks of cancer and lower serum cholesterol (Graham \& Vance, 2003). Legumes such as soybean can also be milled in to flour to make bread (Garcia et al., 1998).

All legumes are characterized by five-petalled flowers with an ovary that ripens to form the distinctive legume pod (Doyle \& Luckow, 2003). The pod develops from a simple carpel and opens along a seam on two sides. At maturity, the two sides may split apart to release seeds 
attached to the seams. Compared to other plant families, legumes exhibit greater floral morphological variation that also extends to fruit type, ranging from tiny single-seeded forms to meter long woody pods. Based primarily on the morphology of flowering plants, legumes are divided into three groups or subfamilies Caesalpiniaceae, Mimosaceae and Papilionaceae (Sprent, 1995; Wojciechowski, 2003). The Papilionaceae have one large petal, two adjacent wing petals on the sides and two bottom petals that join together in a boat-like structure (keel). The flowers of the Caesalpiniaceae are zygomorphic and very variable with five equal petals. The Mimosaceae have small petals, frequently spicate with showy stamens (Heichel \& Vance, 1983; Somasegaran \& Hoben 1994).

Members of the Caesalpiniaceae are mainly tropical and include 162 genera and 1900 species classified in one of four tribes: Cassieae, Caesalpinieae, Cercideae and Detarieae. Of these, only the members of tribes Cassieae and Caesalpinieae (about 30\% of the total number of species in the subfamily) can be nodulated (Allen \& Allen, 1981; Corby et al., 1983; Herendeen et al., 2003). The Mimosaceae include 77 genera and around 3000 species, which mostly occur in subtropical and temperate regions and are classified in three tribes Mimosaceae, Acacieae and Ingeae. The majority of these woody legumes (96\%) are nodulated, and a few have apparently lost their nodulation abilities (Allen \& Allen, 1981; Corby et al., 1983). The Papilionaceae is the largest subfamily with 476 genera and about 14000 species representing 32 tribes (i.e. Swartzieae, Sophoreae, Dipterygeae, Dalbergieae, Abreae, Tephrisieae, Robinieae, Indigofereae, Desmodieae, Phaseoleae, Psoraleeae, Amrpheae, Sesbanieae, Aeschynomeneae, Adesmieae, Galegeae, Carmichaelieae, Hedysareae, Loteae, Coronilleae, Vicieae, Cicereae, Trifolieae, Brongniartieae, Mirbelieae, Bossiaeeae, Podalyrieae, Liparieae, Crotalarieae, Euchresteae, Thermopsideae and Genisteae) (Corby et al., 1983). The members of this subfamily generally represent tropical herbaceous species with around $98 \%$ involved in nodulation and the nitrogen-fixing symbiosis (Allen \& Allen, 1981; Corby et al., 1983).

\subsubsection{The tribe Crotalarieae}

The tribe Crotalarieae is characterized by herbaceous and shrubby legumes with 3-7 foliolate digitate leaves (Corby et al., 1983). The stipule in Crotalarieae is extremely reduced in size while in some genera (e.g. Lebeckia) the stipule is completely absent (Le Roux \& van Wyk, 2007). The tribes Crotalarieae, Podalyrieae and Genisteae are considered to be closely related and include numerous agriculturally important legumes (Corby et al., 1983; Van Wyk \& Schutte, 1995; Sprent, 2007). Based on DNA-based phylogenetic studies, Wojciechowskie (2003) 
demonstrated that the tribe Crotalarieae, together with the tribes Podalyrieae, Genisteae, Thermopsideae, Euchresteae, Sophoreae and Lipariaea form part of the genistoid sensu lato clade which all share a recent common ancestor (Corby et al., 1983; Crisp et al., 2000; Wojciechowski, 2003; Sprent, 2007).

The tribe Crotalarieae includes 16 genera (i.e. Lebeckia, Wiborgia, Rafnia, Aspalathus, Spartidium, Crotalaria, Bolusia, Lotononis, Buchenroedera, Pearsonia, Rothia, Robynsiophyton, Melolobium, Dichilus, Anarthrophyllum and Sellocharis) (Corby et al., 1983). Of these, Lebeckia, Lotononis and Crotalaria are taxonomically very similar as several characters found in some groups of Lotononis are present in Lebeckia and Crotalaria (Van Wyk, 1991). They are mostly centered in Africa and adjoining areas (Corby et al., 1983; Van Wyk, 1991), with Crotalaria species distributed extensively in Africa as well as Asia, Australia and across the Atlantic to South America. Lotononis is centered in southern Africa with some species also occurring in Australia, the Mediterranean region and India. Lebeckia species are mainly indigenous to the western and southern regions of South Africa (Germishuizen \& Meyer, 2003; Boatwright et al 2007; Le Roux \& van Wyk, 2007). In terms of the nodulation abilities of this tribe, most of the indigenous species examined produce indeterminate nodules with apical meristems that form branches (Corby et al., 1983; Sprent, 2001; Sprent, 2007). The Crotalarieae appear to be nodulated by a variety of root nodule bacteria. In addition to nodulation with members of the family Rhizobiaceae, certain species of the African genera Cyclopia and Aspalathus are nodulated by Burkholderia belonging to the $\beta$-Proteobacteria, while certain Crotalaria and Lotononis species develop effective nodules when infected with Methylobacterium belonging to the $\alpha$-Proteobacteria (Sy et al., 2001; Jaftha et al., 2002; Sprent, 2007).

Like many other legumes, those in the tribe Crotalarieae produce a range of secondary compounds that may be toxic. For example, pyrolizidine alkaloids cause chronic poisoning of livestock and quinolizidine causes heart-related diseases, vomiting in humans and teratogenic effects in cattle (Van Wyk et al., 2002). The presence or absence of secondary compounds also represents an important classification criterion for the tribe. Compounds like nutalline, lupanine and sparteine have been identified previously in Lebeckia (Van Wyk \& Verdoorn, 1989a), while the alkaloid, quinolizidine is common in Lebeckia cytisoides (Van Wyk et al., 2002). In the tribe Crotalarieae, Lebeckia differs from other quinolizidine/macrocyclic pyrrolizidine alkaloidbearing genera Buchenroedera, Crotalaria and Lotononis by the absence of $\alpha$-pyrodone 
alkaloids and esters of alkaloids. Van Wyk \& Verdoorn (1989a, b) used this chemotaxonomic evidence to conclude that Buchenroedera and Lotononis are more closely related to Crotalaria than to Lebeckia despite morphological evidence. Members of the Crotalarieae also accumulate large amounts of hydroxylated lupanine-type alkaloids (Lebeckianine) in their seeds (Van Wyk \& Verdoorn, 1989a, b; Van Wyk \& Schutte, 1995).

Several species in this tribe are agriculturally important. For example, plants of the genus Crotalaria are used in green manures and intercropping (Giller, 2001), Aspalathus linearis is the source of the well-known rooibos tea beverage (Van Heerden et al., 2003) and Lotononis bainesii is a valuable forage legume (Yates et al., 2007). The genus Lebeckia is of concern for this study and is presently not commercially exploited but has horticultural and forage potential (see below).

\subsubsection{The genus Lebeckia}

The genus Lebeckia Thunb. was last revised taxonomically by Harvey in 1862, following Bentham (1844). This taxonomic system is however currently being revised (Le Roux \& Van Wyk, 2007; Boatwright et al., 2007) and this study follows the B-E. van Wyk (personal communication) classification of Lebeckia species into the three sections Calobota, Virborgiodes and Stiza with trifoliate leaves and shrubby growth habits and the two sections Lebeckia and Spira with needle-shaped or unifoliolate leaves and suffrutescent or herbaceous growth habits. The shape and size of fruits appear to be the most useful character for distinguishing the species (Le Roux \& van Wyk, 2007). The genus consists of some 33-35 species (listed in Table 1.4) that are mainly indigenous to the western and southern regions of South Africa (Germishuizen \& Meyer, 2003; Boatwright et al., 2007; Le Roux \& van Wyk, 2007). Le Roux \& Schelpe (1997) also mentioned that 35 species of Lebeckia are found in southern Africa with 15 species occurring in Namaqualand. The plants within this genus are tolerant to acidic soils and harsh conditions and also show a high tolerance towards cold temperature and frost. No studies of the symbiotic rhizobia of Lebeckia have been documented prior to the investigation presented in this dissertation.

The largest section is Lebeckia with 12 species (Le Roux \& van Wyk., 2007). They have acicular needle-shaped leaves that are spirally arranged into branches. The reference species for this section is Lebeckia sepiaria (Le Roux \& van Wyk., 2007). They are mainly perennial herbs of height between 0.1-0.7 $\mathrm{m}$ and grow mostly in the Northern Cape and Western Cape provinces. 
The very rare L. wrightii (Germishuizen \& Meyer, 2003; Le Roux \& van Wyk, 2007) also occurs here and is the only species in the section Spira. Many annual species are found in Clanwilliam and Bushmanland in the east of Namaqualand (Le Roux \& Schelpe, 1997).

According to the revision by Boatwright et al. (2007), three species (L. cuspidosa, L. psiloloba and L. pungens) are classified in the shrubby section Stiza. Their heights are around 0.5-2.5 m and they are abundant in the Northern Cape, Eastern Cape and near Bloemfontein in the Free State Province (Germishuizen \& Meyer, 2003). Section Stiza is characterized by plants that are extremely thorny, with unifoliolate leaves (Boatwright et al., 2007). Species in section Calobota are perennial shrubs with heights of $0.15-0.6 \mathrm{~m}$. The section consists of about nine species occurring in the Northern Cape, Western Cape and Eastern Cape Provinces. Section Viborgioides contains perennial shrubs with heights of $0.2-2 \mathrm{~m}$. They develop well in the Western Cape, Free State, Eastern Cape and Northern Cape (Germishuizen \& Meyer, 2003).

Various Lebeckia spp. represent pasture and forage legumes which are well suited to moderate to heavy grazing conditions. Several species, especially L. spinescens, L. multiflora and L. sericea, are recognized as valuable forage plants in natural veldt. Lebeckia spinescens (the name means "becoming spinous") or sand ganna is found in dry clay or granite slopes from Namibia to Malmesbury in the western Cape and in drier Karoo areas to the Eastern Cape (Manning \& Goldblatt, 1996; 1997) and in Namaqualand, Bushmanland, Great Karoo and the southern Cape (Le Roux et al., 1994; Shearing \& van Heerden, 1994) and can survive with relatively little annual rainfall (100-420 mm pa.). It produces bean-pod fruits that have high nutritional value and are very well grazed. Another well grazed species is L. sericea or "blou fluitjies" (Fig. 1.1d) that produces edible flowers and pods and grows in rocky places in Namaqualand and drier areas of the south Western Cape Province (Le Roux \& Schelpe, 1997). Although L. multiflora or "fluitjiesbos" (Fig 1.1c) is not readily grazed by animals, it is valuable in times of drought when even the dry leaves, flowers and pods can be eaten. This species also can be utilized to stabilize old lands and unstable sand (Van Breda \& Barnard, 1991).

\subsection{THE TAXONOMY OF RHIZOBIA}

All nodulating nitrogen-fixing bacteria are part of the Proteobacteria found in the domain Eubacteria. The Proteobacteria represents the second largest group of bacteria and consist of a diverse range of organisms including purple phototrophic, nitrifying and enteric bacteria as well 
as symbiotic and free-living nitrogen-fixing bacteria (Jordan, 1984). All Proteobacteria are Gram-negative and motile by means of flagella. Based on analyses of the highly conserved small subunit 16S ribosomal RNA (16S rRNA) gene sequence, the Proteobacteria are divided into the five groups $\alpha-, \beta$-, $\gamma$-, $\delta$ - and $\varepsilon$-Proteobacteria (Jordan, 1984; Zakhia $\&$ de Lajudie, 2001). Nodulation and nitrogen fixation is restricted to the $\alpha$-Proteobacteria and $\beta$ Proteobacteria. The terms $\alpha$ - and $\beta$-rhizobia were proposed to distinguish rhizobia of the $\alpha$ Proteobacteria and $\beta$-Proteobacteria (Chen et al., 2003a).

At first, bacteria that formed nitrogen-fixing (diazotrophic) nodules on legumes were all assigned to the genus Rhizobium with different species being recognized on the basis of cross-inoculation nodulation specificity (Fred et al., 1932; Young, 1996). However, Fred et al. (1932) recognized that rhizobia could be differentiated into fast-growing and slow-growing groups on laboratory media. Later, Jordan (1984) used DNA composition and other information to divide rhizobia into the fast-growing genus Rhizobium, including species that nodulate legumes such as bean, clover and pea, and the slow-growing genus Bradyrhizobium, including rhizobia that nodulate legumes such as soybean and cowpea. In 1984, Bergey's Manual of Systematic Bacteriology classified the Rhizobiaceae into four genera: Rhizobium, Bradyrhizobium, Agrobacterium and Phyllobacterium (Jordan, 1984). Further phylogenetic studies based on sequence comparisons of the 16S rRNA gene led to classification of the rhizobia into four genera: Rhizobium, Bradyrhizobium, Sinorhizobium and Mesorhizobium, all of which lie in different branches of the $\alpha$-Proteobacteria (Fig. 1.2), and later separation of the genus Rhizobium into six genera (Young et al., 2001).

Like most other bacteria, the classification of nitrogen-fixing bacteria (diazotrophs) is under constant review as new species are discovered and existing species are split or condensed into one (Laguerre et al., 1994). The most recent taxonomy of rhizobia consists of 62 species found in 12 genera of nodule-forming diazotrophic bacteria, nine of which are $\alpha$-rhizobia (Azorhizobium, Bradyrhizobium, Devosia, Mesorhizobium, Methylobacterium, Ochrobactrum, Phyllobacterium, Rhizobium and Sinorhizobium) and three $\beta$-rhizobia (Burkholderia, Cupriavidus and Herbaspirillum) (Moulin et al., 2001; Sawada et al., 2003; Weir et al., 2004; Wolde-Meskel et al., 2005; Weir, 2006). The currently recognized species of rhizobia are listed in Table 1.2, as well as on the phylogenetic trees (Figs. 1.2, 1.3), and are also discussed below. 


\subsection{1 $\alpha$-Rhizobia}

\subsubsection{The genus Rhizobium}

The genus Rhizobium is phylogenetically heterogeneous and consists of more than 20 accepted species (Zakhia \& de Lajudie, 2001; Quan et al., 2005; Willems, 2006; Weir, 2006). The name Rhizobium means 'root living' and was first recognized by Frank in 1889. Rhizobium, Agrobacterium and Allorhizobium are closely related (Willems, 2006) and the latter two genera were incorporated into the genus Rhizobium (Young et al., 2001). Their strains are characterized by fast growth on yeast extract mannitol medium and the production of acid in mineral saltsmannitol medium. The DNA G+C content of the genus is in the range 59-64 mol\% (Jordan, 1984). The finding that the fast-growing $R$. meliloti shares major features with the nonnodulating genus Agrobacterium, led Young et al. (2001) to propose combination of the genera Agrobacterium and Rhizobium into a single genus Rhizobium. The former species of agrobacteria are now classified in to the genus Rhizobium, including Ag. tumefaciens, Ag. radiobacter, Ag. rhizogenes, Ag. rubi and Ag. vitis (Holmes \& Roberts, 1981; Kwon et al., 2005). Many Agrobacterium species are pathogens responsible for tumour or gall-like symptoms on their hosts (Young et al., 2001) and are therefore not considered here in detail.

\section{(a) Rhizobium leguminosarum}

Rhizobium leguminosarum, $R$. trifolii and $R$. phaseoli are now regarded as biovars of a single species $R$. leguminosarum (Jordan, 1984), of which the type strain for this species nodulates members of the legume genus Vicia. The three biovars of $R$. leguminosarum include biovar viciae nodulating the tribe Vicieae (Pisum, Vicia, Lathyrus and Lens); biovar trifolii nodulating Trifolium species (clover) and biovar phaseoli nodulating Phaseolus vulgaris (common bean).

\section{(b) Rhizobium etli and Rhizobium galegae}

Rhizobium etli was proposed after genetic and phenotypic characters of isolates nodulating both alfalfa and beans indicated a group distinct from R. leguminosarum bv. phaseoli (Segovia et al., 1993). R. etli contains two biovars, R. etli bv. phaseoli and R. etli bv. mimosae (Wang et al., 1999a). The strains nodulate and fix nitrogen in association with Phaseolus vulgaris and other legumes such as Mimosa affinis (Brenner et al., 2005). Rhizobium galegae is the symbiont of Galega orientalis and G. officinalis. It appears to be rather specific to these species (Brenner et al., 2005; Young, 1996). 


\section{(c) Rhizobium tropici}

Rhizobium tropici forms symbiotic association with Phaseolus vulgaris and Leucaena spp. The species is distinguished from other rhizobia by DNA-DNA reassociation, multilocus enzyme electrophoresis profiles and biochemical tests, and 16S rRNA sequence comparison (MartinezRomero et al., 1991; Brenner et al., 2005).

\section{(d) Rhizobium gallicum and $R$. giardinii}

The two species were identified by Amarger et al. (1997) using phenotypic differentiation and $16 \mathrm{~S}$ rRNA sequence analysis and are relatively fast-growing. For $R$. gallicum, two biovars, bv. gallicum and bv. phaseoli were identified based on nodulation specificity. Biovar gallicum nodulates and fixes nitrogen in association with Leucaena leucocephala and Phaseolus spp. whereas biovar phaseoli nodulates Phaseolus spp. and Macroptilium atropurpureum. R. giardinii has two biovars, bv. giardinii and bv. phaseoli. Biovar giardinii is symbiotic on Phaseolus spp. other than $P$. vulgaris, as well as $L$. leucocephala and $M$. atropurpureum. $R$. gallicum bv. phaseoli nodulates Phaseolus spp. but is partially efficient in fixing nitrogen in association with L. leucocephala (Brenner et al., 2005).

\section{(e) Rhizobium hainanense}

Unique isolates from the roots of trees, herbs and vines with diverse phenotypic and genotypic characteristics were distinguished as $R$. hainanense, using DNA hybridization studies, cluster analysis of phenotypic features and 16S rRNA sequences (Chen et al., 1997). This fast-growing bacterium was first identified from tropical legumes in Hainan, China. The species also stimulate nodulation on Acacia sinicus, Desmodium gyroides, D. sinuatum and other Desmodium species (Brenner et al., 2005).

\section{(f) Rhizobium huautlense}

The comparative sequence analysis of $16 \mathrm{~S}$ rRNA indicates that $R$. huautlense is closely related to R. galegae (Wang et al., 1998). The two species are differentiated according to multilocus enzyme electrophoresis and DNA-DNA reassociation. $R$. huautlense is a symbiont of Sesbania herbacea, S. rostrata and Leucaena leucocephala, in Mexico (Wang et al., 1998), although the most common symbionts of Sesbania species are Azorhizobium caulinodans as discussed below (Wang et al., 1998). 
(g) Rhizobium mongolense

Rhizobium mongolense shares $99.2 \%$ similarity in its 16S rRNA sequence with $R$. gallicum (Van Berkum et al., 1998). R. mongolense was identified as the symbiont of Medicago ruthenica from the United States and also nodulates Medicago sativa (alfalfa) and fixes nitrogen in Phaseolus vulgaris (Van Berkum et al., 1998). Its presence in the root nodules of these plants contradicts the accepted notion that Sinorhizobium species are the predominant symbionts of the Medigaco species (Le Roux, 2003; Brenner et al., 2005)

\section{(h) Rhizobium sullae}

The rhizobia isolated from Sulla root nodules were previously referred to as $R$. hedysari and were shown to exhibit a high degree of host specificity. 16S rRNA gene phylogenies revealed that the Sulla isolates are related to $R$. gallicum, R. mongolense and R. leguminosarum. Based on the results of this study, the species $R$. sullae was proposed (Squartini et al., 2002).

\section{(i) Rhizobium undicola}

Rhizobium undicola represents the former Allorhizobium undicola (De Lajudie et al., 1998b; Young et al., 2001). It nodulates Neptunia natans an aquatic plant, indigenous to waterlogged areas in Senegal and India (De Lajudie et al., 1998b). Phylogenetically the closest neighbour of R. undicola is the plant pathogen Ag. vitis (Zakhia \& de Lajudie, 2001).

\section{(j) Rhizobium loessense}

Rhizobium loessense was identified from the plant genera Astragalus and Lespedeza in the Loess Plateau, China (Wei et al., 2003). Diverse rhizobia have previously been described from Astragalus that includes Mesorhizobium, Rhizobium and Sinorhizobium. The species $R$. loessense was distinguished from relatives using phenotypic characteristics (Wei et al., 2003).

\section{(k) Rhizobium indigoferae}

Rhizobium indigoferae was isolated from Indigofera host plants in the Loess Plateau China. As with other bacteria, a polyphasic approach (SDS-PAGE, DNA-DNA reassociation, 16S rRNA sequencing) was used to characterize the species. Phylogenetically it appears to be closely related to $R$. mongolense and $R$. yuanglingense (Wei et al., 2002). 


\section{(l) Rhizobium yuanglingense}

The species was isolated and described by Tan et al. (2001). The isolates were collected in arid and semi arid regions in China from the wild legumes Amphicarpaea trisperma, Coronilla varia and Gueldenstaedtia. R. yuanglingense produces ineffective nodules on Phaseolus vulgaris though it does not nodulate Galega orientalis and Leucaena leucocephala (Tan et al., 2001).

\section{(m) Rhizobium lusitanum}

Rhizobium lusitanum was isolated in Portugal from nodules of Phaseolus vulgaris, a promiscuous legume that forms symbiotic associations with most of the species within the Rhizobiaceae family. $R$. lusitanum is closely related to $R$. rhizogenes and $R$. tropici on the $16 \mathrm{~S}$ rRNA phylogenetic trees. It induces effective nodules on Phaseolus vulgaris, Macroptilium atropurpureum, Leucaena leucocephala and ineffective nodules on Medicago sativa (Valverde et al., 2006).

\subsubsection{The genus Bradyrhizobium}

Initially, the slow-growing B. japonicum was the only recognized species of the genus although all soybean symbionts and other slow-growing rhizobia were also placed in the genus (Jordan, 1984; Young \& Haukka, 1996). The cells of members of this genus are non-spore forming and motile by one polar or subpolar flagellum. The colonies are circular, opaque, rarely translucent, white and convex becoming visible five or more days after inoculation. They produce an alkaline reaction in mineral salts medium containing mannitol or other carbohydrates (Jordan, 1984).

Among the rhizobia in the family Rhizobiaceae, Bradyrhizobium is phylogenetically distinct from the more closely related genera Rhizobium, Mesorhizobium and Sinorhizobium (Van Berkum \& Eardley, 1998). The G+C content of the Bradyrhizobium genus is 57-63 mol \%. The genus currently contains seven species; $B$. japonicum, $B$. elkanii, $B$. liaoningense, $B$. yuanmingense, B. betae, B. canariense and B. denitrificans (Jordan, 1984; Kuykendall et al., 1992; Xu et al., 1995; Yao et al., 2002; Rivas et al., 2004; Vinuesa et al., 2005b; Van Berkum et al., 2006). Within the above species, only B. betae is not associated with nodulation and nitrogen-fixation but rather with tumour-like deformations of the roots of Beta vulgaris (Rivas et al., 2004). Undescribed strains of Bradyrhizobium are known as Bradyrhizobium spp., followed 
by the name of the legume host in parentheses e.g. Bradyrhizobium strain (Arachis) (Van Rossum et al., 1995; Young, 1996).

\section{(a) Bradyrhizobium japonicum and B. elkanii}

Both species will effectively nodulate soybean (Glycine max). Soybean B. japonicum is sensitive to acid (Vinuesa et al., 2005b). Among B. japonicum strains two DNA homology groups were initially recognized on the basis of fatty acid analysis, antibiotic resistance and DNA-DNA hybridization (Jordan, 1984; Graham et al., 1991). Hollis et al. (1981) showed that strains belonging to the DNA homology group II of B. japonicum, showed 30\% homology with the type strain of $B$. japonicum. They suggested that these strains represent a distinct species and subsequently B. elkanii was proposed by Kuykendall et al. (1992) for this subgroup of soybean bradyrhizobia, based on DNA homology, RFLP analysis, 16S rRNA sequence data and antibiotic resistance profiles (Vinuesa et al., 2005b).

\section{(b) Bradyrhizobium liaoningense}

Bradyrhizobium liaoningense, proposed by Xu et al. (1995), was identified from soybean and the related Glycine soja using DNA-DNA hybridization, $\mathrm{G}+\mathrm{C}$ analysis and partial 16S rRNA sequence analysis. Colonies of this species are $0.2-1.0 \mathrm{~mm}$ in diameter after 7-14 days growth on yeast mannitol agar, compared with 5-7 days for other Bradyrhizobium species (Xu et al., 1995).

\section{(c) Bradyrhizobium denitrificans}

Bradyrhizobium denitrificans represents the former species Blastobacter denitrificans (Hirsch \& Muller, 1985; Van Berkum et al., 2006). It represents fresh water bacteria that nodulate the water plant Aeschynomene indica (Van Berkum \& Eardley, 2002). The amino acid sequences of certain photosynthetic genes of $B$. denitrificans are identical to those of bradyrhizobial isolates with which they also share the ability to propagate by budding.

\section{(d) Bradyrhizobium canariense}

Bradyrhizobium canariense got its name from the fact that the species is found in the Canary islands nodulating endemic shrub legumes. Strains of $B$. canariense are highly acid-tolerant (Vinuesa et al., 2005b) and nodulate diverse species in the tribes Genisteae and Loteae. B. canariense also fixes nitrogen on the promiscuous legume Macroptilium atropurpureum and does not nodulate Glycine max (Vinuesa et al., 2005b). B. canariense can be distinguished from other Bradyrhizobium species by a combination of genotypic, physiological and ecological 
characteristics, most appropriately by DNA-DNA hybridization. B. canariense strains form a monophyletic cluster that appears to be most closely related to B. japonicum (Vinuesa et al., 2005b).

\section{(e) Bradyrhizobium yuanmingense}

Bradyrhizobium yuanmingense was isolated from the root nodules of Lespedeza species growing in China (Yao et al., 2002). The word "yuanmingense" refers to the royal garden Yuanmingyuan in Beiljing, China, where the bacteria was isolated. Again, a polyphasic approach (DNA-DNA hybridization, 16S rRNA sequencing and nodulation tests with selected host legumes) was used to characterize B. yuanmingense (Yao et al., 2002).

\subsubsection{The genus Sinorhizobium}

The genus Sinorhizobium was originally proposed by Chen et al. (1988) for the group of fastgrowing rhizobia that nodulate lucerne, soybean and various Medicago spp. (Scholla \& Elkan, 1984; Chen et al., 1988). De Lajudie et al. (1994) emended the genus Sinorhizobium, and reclassified $R$. meliloti as $S$. meliloti and also transferred $R$. fredii to the Sinorhizobium genus. Due to various technicalities regarding the International Code of Nomenclature of Bacteria, Sinorhizobium is considered to be synonymous with the genus Ensifer (Martens et al., 2007). Sinorhizobium includes 11 species, with $S$. meliloti as the type strain.

\section{(a) Sinorhizobium fredii and $S$. xingiangensis}

These two species can effectively nodulate soybean (Glycine max, G. soja), cowpea (Vigna unguiculata) and pigeon pea (Cajanus cajan). Two groups within $S$. fredii are recognized based on DNA-DNA hybridization and serology, i.e. chemovars fredii and siensis (Scholla \& Elkan, 1984). Within the genus Sinorhizobium, 16S rRNA phylogeny is sufficient to distinguish between S. fredii and S. meliloti (see below) (Jarvis et al., 1992). However, S. fredii and $S$. xingiangensis share $100 \% 16 \mathrm{~S}$ rRNA similarity. The fast-growing S. xinjiangensis was associated with soybean nodules in Xinjiang, China (Chen et al., 1988).

\section{(b) Sinorhizobium meliloti and S. medicae}

Sinorhizobium meliloti is a symbiotic partner of three legume genera: Medicago, Melilotus and Trigonella (Eardly et al., 1990; Rome et al., 1996; Young, 1996). This species could be distinguished from other members of this genus by electrophoretic protein profiles, DNA-DNA 
hybridization and 16S rRNA sequences (De Lajudie et al., 1994). S. medicae also nodulates Medicago species, and is distinguishable from $S$. meliloti using DNA-DNA hybridization studies among others (Rome et al., 1996).

\section{(c) Sinorhizobium saheli and S. terangae}

In 1994, the two species $S$. saheli and S. terangae were accepted as new species (De Lajudie et al., 1994). The species were obtained from different Acacia and Sesbania hosts in the Sahel area of Senegal. S. saheli strains are capable of nodulating Sesbania species found in the Sahel area and are also found in association with Acacia, Leucaena and Neptunia species (Boivin \& Giraud, 1999). On the basis of $16 \mathrm{~S}$ rRNA sequences phylogeny, the strains cluster with $S$. meliloti and $S$. fredii (Fig. 1.2).

\section{(d) Sinorhizobium arboris, S. kostiense and S. morelense}

These three species have the ability to induce the development of root nodules on Acacia senegal and Prosopis chilenses originating from Kenya and Sudan (Nick et al., 1999a). Phylogenetically S. arboris and S. kostiense group separately from S. morelense (Fig. 1.2) (Nick et al., 1999b). The latter are associated with the legume Leucaena leucocephala. They may be identified using RFLP analysis of $16 \mathrm{~S}$ rRNA together with Southern hybridization of nifH and nodDAB (Wang et al., 1999c; Wang et al., 2002).

\section{(e) Sinorhizobium kummerowiae}

Sinorhizobium kummerowiae was isolated from the drought-tolerant perennial Kummerowia stipulacea in China (Wei et al., 2002), which is used as green manure and for preventing soil erosion (Allen \& Allen, 1981). 16S rRNA gene analysis indicated that the isolates from $K$. stipulacea are related to $S$. fredii, S. xinjiangense, S. saheli and S. terangae.

\section{(f) Sinorhizobium americanus}

Sinorhizobium americanus was isolated from the nodules of Acacia acatlensis in Mexico (Toledo et al., 2003). The species differ from other Sinorhizobium species in terms of DNADNA hybridization, $16 \mathrm{~S}$ rRNA sequences and nif $H$ gene sequences.

\subsubsection{The genus Mesorhizobium}

This genus was proposed by Jarvis et al. (1997), and its members share the property of acid production with species of Rhizobium and Sinorhizobium (Van Berkum \& Eardley, 1998). There 
are 12 recognized species within the genus with $M$. plurifarium being the type strain (Young, 1996) (Table 1.2). The genus is intermediate in growth between the fast-growing Agrobacterium-Rhizobium-Sinorhizobium group and the slow-growing genera Azorhizobium and Bradyrhizobium (Jarvis et al., 1997). The name Mesorhizobium implies intermediate growth rate and phylogenetic position (Fig. 1.2). Most of the species within this genus were former members of the Rhizobium genus, including Mesorhizobium loti (formerly $R$. loti), Mesorhizobium huakuii (formerly R. huakuii), Mesorhizobium ciceri (formerly R. ciceri), Mesorhizobium mediterraneum (formerly R. mediterraneum) and Mesorhizobium tianshanense (formerly R. tianshanense) (Young, 1996; Jarvis et al., 1997). They were however separated from the fast-growing rhizobia by properties such as the location of symbiotic genes, 16S rRNA phylogeny (Fig.1.2) and DNA homology.

\section{(a) Mesorhizobium loti and M. huakuii}

Mesorhizobium loti form effective nodules on species of Lotus, Lupinus and Anthyllis (Young, 1996). The Mesorhizobium isolates from the nodules of the legume genus Astragalus revealed a distinct DNA homology group that carry their symbiotic genes on plasmids and not on chromosomes, as is the case with other Mesorhizobium (Chen et al., 1991). The isolates were identified as M. huakuii.

\section{(b) Mesorhizobium ciceri and M. mediterraneum}

The chickpea symbionts are classified as Mesorhizobium species regardless of generation time and they constitute a separate branch closely related to but clearly different from M. loti. Two phylogenetic lineages (groups A and B) were identified, with the more homogenous group B representing M. ciceri (Nour et al., 1994). Group A, on the other hand displayed great heterogeneity (Nour et al., 1994). Based on genomic studies conducted in 1995, it was shown not to be related to any other species, including M. ciceri (Nour et al., 1995). Group A was named M. mediterraneum and differentiated from other mesorhizobia by $16 \mathrm{~S}$ rRNA sequence, DNA-DNA hybridization and fatty acid analysis (Nour et al., 1995).

\section{(c) Mesorhizobium tianshanense}

Chen et al., 1995, used numerical taxonomy to analyze a number of strains isolated from various leguminous plants in the arid saline environment in the Tianshan Mountains of Xinjiang China. A large number of the fast-growing isolates corresponded to Rhizobium species whereas moderate slow-growers were described as M. tianshanense. M. tianshanense nodulate Glycine 
max, Glycyrrhiza spp. and Caragona polourensins and does not induce nodules on the promiscuous legumes Macroptilium atropurpureum, Vigna unguiculata, Phaseolus vulgaris, or Pisum sativum (Chen et al., 1995).

\section{(d) Mesorhizobium plurifarium}

Mesorhizobium plurifarium was isolated from the root nodules of tropical Acacia species from Senegal, and Lotus species (De Lajudie et al., 1994). De Lajudie et al. (1998a) performed a taxonomic investigation using techniques such as REP-PCR, 16S rRNA sequencing and DNADNA hybridization, which led to the identification of the species $M$. plurifarium (De Lajudie et al., 1998a). M. plurifarium showed higher similarities of their 16S rRNA sequences to M. loti, M. haukii and M. mediterraneum. M. plurifarium is not host specific, having been isolated from Acacia spp., Prosopis spp., Leucaena spp., Lotus spp., Cicer spp., Medicago spp., Sesbania spp., Trifolium spp., Phaseolus spp., Glycine spp. and related genera (De Lajudie et al., 1998; Wang et al., 2004).

\section{(e) Mesorhizobium amorphae and M. chacoense}

Mesorhizobium amorphae is the rhizobial symbiont of the legume shrub Amorpha fruticosa (Wang et al., 1999b). The legume is native to the south eastern United States and has various agricultural applications including soil erosion control, green manure and biological control (Wang et al., 1999b). The bacterium M. chacoense was described after determining the diversity of rhizobia associated with Prosopis chilensis occurring in diverse geographical regions in central Argentina (Velazquez et al., 2001).

\section{(f) Mesorhizobium albiziae}

Mesorhizobium albiziae nodulates Albizia kalkora, and also forms effective nodules on A. julibrissin, Glycine max, Leucaena leucocephala, Phaseolus vulgaris and several other legumes. The species was discovered in subtropical regions of China (Wang et al., 2007). It varies from previously identified mesorhizobia with unique genomic characteristics (Wang et al., 2007).

\section{(g) Mesorhizobium septentrionale and M. temperatum}

Mesorhizobium septentrionale and M. temperatum were isolated from Astragalus adsurgens growing in northern regions of China (Gao et al., 2004). The name M. temperatum refers to temperate regions in China where isolated. These two species differ significantly in terms of DNA-DNA homology and nodA sequences (Gao et al., 2004). 


\section{(h) Mesorhizobium thiogangeticum}

This species was isolated from the root nodules of Clitoria ternatea, which is a thin herbaceous legume from the Gangetic plains of India. M. thiogangeticum is the first member of Mesorhizobium found to oxidize sulphur (Ghosh \& Roy, 2006). Its 16S rRNA and recA sequences were similar to those of other Mesorhizobium, but were most similar to M. loti, M. plurifarium, M. amorphae and M. chacoense (Ghosh \& Roy, 2006).

\subsubsection{The genus Azorhizobium}

Azorhizobium produce nitrogen-fixing nodules on the stem and roots of Sesbania rostrata (Dreyfus et al., 1988) and its symbiotic association are important in tropical agriculture (Rinaudo et al., 1991). Azorhizobium also assimilates dinitrogen in the free-living state (Dreyfus et al., 1988). Following numeric analysis of phenotypic characters, DNA-DNA hybridization and protein comparison (Dreyfus et al., 1988), this genus was introduced to include rhizobia that have intermediate to fast growth rates. Phylogenetically, Azorhizobium is most closely related to Xanthobacter and Bradyrhizobium.

\section{(a) Azorhizobium caulinodans}

Azorhizobium caulinodans is the type species of the genus and nodulates and fix nitrogen only on Sesbania rostrata (Dreyfus et al., 1988; Rinaudo et al., 1991). The species is very unique in that it grows fast in the free-living state at the expense of dinitrogen as its sole source of nitrogen, which is an essential feature distinguishing it from other rhizobial genera. On $S$. virgata, Az. caulinodans does not complete nodule formation, as pseudonodules are produced (Dreyfus et al., 1988; De Souza Moreira et al., 2006).

\section{(b) Azorhizobium doebereinerae}

This species was formerly named Azorhizobium johannae and is a microsymbiont of the woody plant Sesbania virgata native to Brazil (De Souza Moreira et al., 2006). Az. doebereinerae, nodulates Macroptilium atropurpureum, Phaseolus vulgaris, but with reduced nitrogen fixation. It does, however, only produce pseudonodules on S. rostrata, (De Souza Moreira et al., 2006). Overall, this species is genotypically and phenotypically very similar to Az. caulinodans. 


\subsubsection{The genus Phyllobacterium}

The genus Phyllobacterium includes five nodulating species. Mantelin et al. (2006) descriped the four new species P. bourgognense, P. infrigiyense, P. leguminum and P.brassicacearum, which were originally isolated from root nodules of Brassica napus (in France), Argyrolobium uniflora, Astragalus algerianus and Lathyrus numidicus (in southern Tunisia), respectively. $P$. trifolii forms symbiotic associations with plants of the genus Trifolium pratense and Lupinus albus (Valverde et al., 2005). Phylogenetically, members of this genus are most closely related to Mesorhizobium species (Young et al., 2001).

\subsubsection{The genus Ochrobactrum}

The genus Ochrobactrum was first described by Holmes et al. (1988) from family Brucellaceae, of the $\alpha$-Proteobacteria (Fig 1.2). The genus Ochrobactrum currently comprises 11 species, with Ochrobactrum lupini and $O$. cytisi as the first species found to possess rhizobial properties within the genus. O. lupini was originally isolated from Lupinus albus plant nodules in Argentina, and can also nodulate other Lupinus plants from several geographical origins (Trujillo et al., 2005). O. cytisi was isolated from the root nodules of Cytisus scoparius in Spain and is named after this host plant (Zurdo-pineiro et al., 2007).

\subsubsection{Methylobacterium nodulans}

The genus Methylobacterium consists of a variety of pink pigmented facultative methylotrophic (PPFM) bacteria that utilize carbon as their energy source (Green, 1992). The only exception is M. nodulans, described as a facultative methylotropic unpigmented isolate from Crotalaria spp. that utilizes methanol and formate as sole carbon source (Sy et al., 2001; Jourand et al., 2004). In 2002, Jaftha et al. characterized the red-pigmented rhizobia from Lotononis bainesii in South Africa as Methylobacterium isolates with 16S rRNA sequences that were $98 \%$ similar to that of M. nodulans. Their common feature is that their original host plants Crotalaria and Lotononis belong to the same tribe. PPFM Methylobacterium isolates have been also isolated from water and leaf surfaces (Holland, 1997).

\subsubsection{Devosia neptuniae}

The genus Devosia was initiated by Nakagawa et al (1996) and belongs to the family Hyphomicrobiaceae. Six species are classified in the genus Devosia, D. insulae, D. limi, D. neptuniae, D. riboflavina, D. soli and D. subaequoris (Euzéby, 2008). Devosia spp. are mostly 
soil and water bacteria (Nakagawa et al 1996). D. neptuniae is the first Devosia species found to fix nitrogen in symbiosis with plants (Rivas et al., 2003). It nodulates and fix nitrogen on Neptunia natans, an aquatic legume in India (Rivas et al., 2003), as opposed to the N. natans from Senegal in Western Africa that form symbiotic associations with Rhizobium undicola (De Lajudie et al., 1998b; Young et al., 2001).

\subsection{2 $\beta$-Rhizobia}

It recently became evident that the ability to induce nodules on the roots (or stems) of plants is not restricted to the $\alpha$-Proteobacteria (Chen et al., 2003; Chen et al., 2006; Barrett \& Parker, 2006). It is now commonly accepted that some genera in the $\beta$-Proteobacteria are also able to nodulate and establish effective nitrogen-fixing symbioses with legumes. The terms $\alpha$ - and $\beta$ rhizobia were proposed to distinguish rhizobia of the $\alpha$-Proteobacteria and $\beta$-Proteobacteria (Chen et al., 2003). Various legumes and environments remain to be explored and it is, therefore, highly likely that further characterization of rhizobia will reveal an even greater diversity (Chen et al., 2003).

\subsubsection{The genus Burkholderia}

Burkholderia species are common soil and rhizosphere inhabitants, whilst others are plant and human pathogens (Baldani et al., 2000; Eastrada-de los Santos et al., 2001; Goris et al., 2004; Payne et al., 2005). The genus was created in 1992 through the transfer of seven Pseudomonas species to this genus. The genus currently includes about 30-34 species (Coenye et al., 2001; Coenye \& Vandamme, 2004; Payne et al., 2005; Perin et al., 2006). A number of Burkholderia spp. can effectively nodulate legumes and have predominantly been found to nodulate mimosoid legumes (Moulin et al., 2001; Sy et al., 2001; Perin et al., 2006). Currently, five Burkholderia spp. are known to nodulate legumes. These are B. tuberum, B. phymatum, B. caribensis, B. mimosarum and B. nodosa, while a number are only known to fix nitrogen (e.g. B. unamae, B. vietnamiensis, B. tropica and B. kururiensis) (Moulin et al., 2001; Vandamme et al., 2002; Chen et al., 2003a; Reis et al., 2004; Caballero-Mellado et al., 2004; Chen et al., 2006; Elliott et al., 2007; Chen et al., 2007). They are however not necessarily related to one another as they are scattered throughout the 16S rRNA phylogeny of Burkholderia (Fig. 1.3) (Coenye \& Vandamme, 2004. The various symbiotic species of this genus are described below. 


\section{(a) Burkholderia tuberum and Burkholderia phymatum}

The first Burkholderia species identified as $\beta$-rhizobia were B. tuberum and B. phymatum (Moulin et al., 2001; Coenye \& Vandamme, 2004). B. phymatum was isolated from Machaerium lunatum in French Guiana and has been shown to nodulate and fix nitrogen on almost 30 Mimosa spp. (Sprent, 2007). B. tuberum was isolated from Aspalathus carnosa in South Africa, but recent studies indicate it also nodulates several Cyclopia spp. (Elliott et al., 2007; Sprent, 2007). Both these species also nodulate the promiscuous legume Macroptilium atropurpureum (siratro) (Moulin et al. 2001). The identity of B. tuberum and B. phymatum were confirmed with 16S rRNA sequencing and polyphasic taxonomy (Vandamme et al., 2002).

\section{(b) Burkholderia caribensis}

This species effectively nodulates Mimosa diplotricha and M. pudica (Chen et al., 2003a) and was identified using a polyphasic approach. The name caribensis refers to the fact that it originates from the Caribbean Islands (i.e. Martinique in French West Indies) (Achouak et al., 1999). Bacterial strains of $B$. caribensis are also responsible for the formation of microaggregates in an island vertisol that consists of very high clay content (Achouak et al., 1999, Vandamme et al., 2002).

\section{(c) Burkholderia mimosarum and B. nodosa}

Burkholderia mimosarum and B. nodosa were both isolated from Mimosa species. $B$. mimosarum was isolated from root nodules of M. pigra and M. scabrella from Taiwan and South America (Chen et al., 2006). B. nodosa was extracted from the nodules of woody legumes $M$. bimucronata and M. scabrella in Brazil (Chen et al., 2007). Based on 16S rRNA sequence results, these species are always found close to one another and also to the nitrogen-fixing $B$. tropica and B. unamae. Whole-cell protein analyses distinguished them readily from other Burkholderia species (Chen et al., 2006).

\subsubsection{Herbaspirillum Iusitanum}

Herbaspirillum lusitanum was isolated in Portugal from the root nodules of Phaseolus vulgaris (Valverde et al., 2003). It is the only diazotrophic species within the genus Herbaspirillum with the ability to form beneficial symbiotic associations with Oryza officicalis (Fig. 1.2). The species may be distinguished from other Herbaspirillum species using phenotypic tests and antibiotic resistance to antibiotics (Valverde et al., 2003). 


\subsubsection{Cupriavidus taiwanensis}

Many of the bacterial strains associated with Mimosa nodules were originally placed in the genus Ralstonia (Chen et al., 2003b). They induce the formation of indeterminate nodules on most Mimosa species within 15 days of inoculation. Yabuuchi et al., (1995) proposed that the genus Ralstonia incorporate the previous species, Alcaligenes eutrophus, Pseudomonas solanacearum and Pseudomonas picketii. The emendation of Cupriavidus was based on the work presented by Makkar and Casida (1987) with several modifications (Vandamme \& Coenye, 2004). All the members of the former genus Ralstonia were reclassified as Cupriavidus (Vandamme \& Coenye, 2004). The only rhizobial species of this genus is $C$. taiwanensis (Chen et al., 2003b). It is an effective symbiont of $M$. pigra and $M$. pudica in Taiwan and the association induces improved plant growth when compared to other rhizobia (Barrett \& Parker, 2006).

\subsection{METHODS FOR CLASSIFYING RHIZOBIA}

The ability to nodulate a legume is still the basic criterion for deciding whether or not a bacterial isolate belongs to the different rhizobial groups. Early attempts at classification were based on specificity amongst rhizobia for their respective host plants and led to the concept of crossinoculation groups in which each group of legume host species could only be nodulated by a corresponding group of compatible rhizobial strains, while incompatible strains from another group would be incapable of nodulation. This system provided the basis for initial classification of species in the genus Rhizobium, although inconsistencies such as differences in host specificity of strains within species and overlapping nodulation ranges between groups later showed that this system was not practical. The fact that evolution of rhizobia predates and differs from that of their respective symbiotic genes explains why this system had little chance of success (Sprent, 2007). Nevertheless the cross-inoculation concept still has practical value as it provides the foundation for selecting suitable effective inoculant strains for particular legume crops.

Cross-inoculation was often used together with other differentiation methods for the identification of rhizobial strains. These included cultural characteristics such as type and rate of growth on Congo red yeast mannitol medium, as well as serological and bacteriophage crossreactivity (Somasagaren \& Hoben, 1994). Other characterization techniques include substrate 
utilization, enzyme linked immunosorbent assay (ELISA), antibiotic resistance, plasmid profile analysis, multilocus enzyme electrophoresis and fatty acid analysis (Vandamme et al., 1996; Niemann et al., 1997; Young \& Haukka 1996). Bacterial differentiation based on phenotypic traits generally requires the use of live cultures. In contrast, analysis based on DNA has enabled dramatic advances in unravelling relationships amongst bacteria and other living organisms. Nevertheless, phenotypic traits are useful when used in combination with genotypic DNA analysis in polyphasic classification approaches described below in section 1.5.1 (Rossum et al., 1995). Table 1.1., lists the phenotypic and genotypic methods employed for microbial identification and classification.

A variety of DNA-based methods have emerged for the identification of rhizobia. These include genomic restriction fragment length polymorphisms (RFLP), random amplified polymorphic DNAs (RAPDs) using random probes, rRNA analysis, DNA-DNA hybridization, Southern blot analysis of nitrogen-fixation (nif) and nodulation (nod) genes and polyacrylamide gel analysis of total proteins (Richardson et al., 1995; Mathis \& McMillin, 1996; Thies et al., 2001). The main reason for this shift in focus is associated with the fact that DNA-based approaches are relatively easy to apply, robust and resolve even closely related species (Thies et al., 2001). Also, the identity of the rhizobia in specific soils can be determined without having to culture the rhizobia from nodulated legumes (Thies et al., 2001). In the following sections, I briefly discuss some of the main DNA-based and non-DNA-based methods used in the polyphasic taxonomy of rhizobia.

\subsubsection{Non-DNA-based methods}

Phenotypic methods or non-DNA based techniques usually require the use of live cultures and do not directly involve genetic material for the classification of rhizobia. These techniques mainly include morphological and biochemical characters. Morphology encompasses both cellular and colonial characteristics of bacteria, while biochemical features include traits such as growth temperature, optimum $\mathrm{pH}$, substrate utilization, salt concentration and activities of various enzymes (Bergerson, 1980). Rhizobial strains also have been characterized phenotypically using host specificity, antibiotic resistance, plasmid profile analysis, multilocus enzyme electrophoresis (MLEE) and fatty acid analysis (Vandamme et al., 1996; Niemann et al., 1997; Young \& Haukka, 1998). Computerized numerical analysis of phenotypic data allows for the comparison of large numbers of phenotypic traits for large numbers of strains. 


\subsubsection{Substrate utilization and antibiotic resistance}

Carbohydrate utilization properties are of taxonomic significance (Somasegaren \& Hoben, 1994). Resistance to low levels of antibiotics is also used for rhizobial strain characterization and identification. To perform these tests, rhizobial cultures are inoculated in media containing various carbohydrates and antibiotics. Generally, species of Rhizobium have the ability to utilize a wider range of carbohydrates than Bradyrhizobium strains. The introduction of automated systems such BIOLOG and API provides a much more convenient approach for conducting numerous sole carbon source tests in a single microtitre plate. Typically, the utilization of carbon is detected by colour change in a tetrazolium indicator dye and automated spectrophotometers enable plates to be scanned, and data analyzed, which can then be compared to library databases of strains (Rinaudo et al., 1991; McInroy et al., 1999; Young, 1996; Sadowsky \& Graham, 2004). The traditional application of these tests are however laborious, time-consuming and not always useful for identifying isolates to species level.

\subsubsection{Morphological characters}

Morphological characters are generally regarded as less reliable or more difficult to apply experimentally than serological differences (see below). Morphology does however often provide secondary "back-up" for strain and species diagnoses. Differences in colony type may also provide a direct means of strain recognition. For example, the unique red colony of rhizobial strains that nodulate Lotononis can easily be distinguished from other rhizobial cultures (Jaftha et al., 2002). Overall, morphological traits are of little use in identifying strains within a species, but are of some value for recognizing strains from different species or genera (Bergerson, 1980).

\subsubsection{Serological methods}

Antisera are useful for identifying many strains of microorganisms. Exceptions arise when permanent and acquired sharing of antigenic similarities are encountered, for example, loss of antigenic specificity by strains of Sinorhizobium meliloti after prolonged culturing (Bergerson, 1980). Serological methods have been applied extensively in the genus Rhizobium. The antigens and antibodies may be detected by agglutination and immunodiffusion within the nitrogen-fixers. The agglutination test is sensitive, but more complex to use and does not give clear distinctions between reactions of antigenic identity compared to immunodiffussion. In gel immunodiffusion two wells are cut in an agar gel in a Petri dish and a bacterial suspension is placed in one well 
and the antiserum in the other well (Somasegaren \& Hoben 1994). The agglutination reaction is based on the aggregation of bacterial cells or particle antigens caused by antibody molecules binding to their surfaces and cross-linking them whereas immunodiffusion bands or lines in agar are formed by precipitation of soluble antigens and antibodies, when they meet after migration towards one another (Bergerson, 1980). Immunodiffusion is used for the detection of soluble antigens and for determining the identities of strains occupying nodules (Somasegaren \& Hoben 1994).

\subsubsection{Biochemical properties}

The main biochemical properties adaptable for identification purposes are those involving fermentation or specific enzyme functions, whereby colonies or agar zones surrounding colonies show distinctive colour reactions after treatment with certain reagents. Activity of specific enzymes in colonies is typically detected by the appearance of clear halos around these colonies, against an opaque, coloured, or fluorescing background of the substrate over the agar surface. For example, to detect nucleases the agar surface is flooded with dilute hydrochloric acid to precipitate undegraded nucleic acid incorporated in the agar. Similar procedures are available for cellulose, urease and other degradative enzyme activities. These methods have been used for diazotrophic nitrogen-fixing bacteria, but are usually not useful for diagnosing rhizobial isolates to the species-level.

The determination of moles guanosine $(\mathrm{G})$ and cytosine $(\mathrm{C})$ in DNA is a standard criterion in bacterial species descriptions. Amongst prokaryotes, mole \% $\mathrm{G}+\mathrm{C}$ content ranges between 24$76 \%$, while within a well-defined species the range is less than $3 \%$ and less than $10 \%$ in a genus (Vandamme et al., 1996). Those organisms with different base composition will have few DNA sequences in common and are likely to be distantly related. Rhizobium species usually have mole \% G+C values in the order of 59-64 \%, Azorhizobium 66-68\%, Bradyrhizobium are intermediate with 61-65 1\% (Graham et al., 1991), whereas Sinorhizobium (De Lajudie et al., 1994) and Mesorhizobium (Jarvis et al., 1997) have mole \% G+C values of 60.8-65.7\% and 59$64 \%$ respectively.

\subsubsection{DNA-based methods}

Techniques employed before the advent of DNA-based methods were limited in a variety of ways. These included restrictions on the number of strains studied, the labour intensity of many 
techniques e.g. protein profiles and the lack of convenient methods to characterize the nature of indigenous rhizobial populations (Thies et al., 2001). Most selection protocols used in the past did not incorporate strain markers and hence there was a high probability of duplicating genotypes. The application of DNA-based methods are associated with a number of additional advantages, in that they often are less time consuming, provide stable information and reflect phylogenetic relationships. The application of these approaches, therefore, has led to significant advances in rhizobial identification and phylogenetic studies.

Each method enables a certain level of phylogenetic classification depending on the primers used and/or genomic regions targeted (Fig. 1.4). For example, analysis of the intergenic 16S-23S rRNA site enables differentiation at genus and species levels while other methods, as mentioned, are more efficient at species-strain levels. (Rademaker \& de Bruijn, 1997). The choice of a molecular typing method therefore depends upon the needs, level of resolution and resources of the laboratory.

In this review, I distinguish between the information generated for whole genomes and that for specific loci. Although both of these approaches are associated with its own set of caveats, the application of both hold significant advantages for studying the diversity of rhizobial populations, and the classification of species and strains. For example, whole genome analyses methods allow the researcher to take into account phenomena such as horizontal gene transfer (HGT) and provide a more complete picture of the diversity within and among species. In the case of rhizobia it is crucially important to also consider HGT as it plays an important role in the evolution of their symbiotic/nodulation abilities and host range (Moulin et al., 2004). However, a major disadvantage of these methods is that they often produce results that are not clear-cut, e.g. AFLPs and DNA-DNA hybridization often do not reveal exact species boundaries (Willems et al., 2001). Although this is also true to some extent for specific loci, the analyses of specific loci are in many cases easier and less labour intensive. The analyses of specific loci also do not take into account the horizontal gene transfer phenomenon. As a result, most studies employ the genetic information for several specific loci (for genes encoding both house keeping functions and symbiotic properties), as well as a range of other data types required for a polyphasic approach (see below) (Parker et al.; 2002; Weir et al.; 2004; Stepkowski et al.; 2005, Vinuesa et al.; 2005b, Barrett \& Parker, 2006) 


\subsubsection{Analyses of whole genomes using fingerprinting methods}

\section{(a) RFLP and Southern hybridization}

DNA fingerprinting technology relied on RFLP analysis of total genomes for years before PCR was developed. This technique involves digestion of total genomic DNA extracted from a specific organism with restriction endonucleases, which produces a complex pattern of bands that are typically revealed by Southern analysis (Vandamme et al., 1996). This technique has been widely applied for studying bacteria as it provides information at the species, subspecies or strain level. The main drawback of this fingerprinting approach is that it is extremely timeconsuming and laborious. As a result it is not used widely anymore and has in most cases been substituted with PCR-based methods.

\section{(b) RAPD, ERIC and REP}

In 1990, two papers appeared describing the RAPD technique (Williams et al., 1990; Welsh \& McClelland, 1990) as a way to rapidly generate genomic fingerprints for a specific organism. This method involves PCR amplification of genomic DNA using short arbitrary primers with 910 bases (Doyle et al., 1993; Micheli \& Bova, 1996). According to Mathis \& McMillin, (1996), variations in DNA structure of Bradyrizobium strains are observed when using RAPD-PCR with GC-rich arbitrary primers (e.g. CRL7) (Mathis \& McMillin, 1996; Botha et al., 2004; Law et al., 2007). This approach is routinely used for the authentication of inoculant strains, identification of predominant nodulating strains from particular field sites, assessment of genetic diversity and relatedness amongst field populations of rhizobia (Richardson et al., 1995; Schneider, \& de Bruijn, 1996; Botha et al., 2004; Law et al., 2007).

REP, ERIC and BOX PCR work on the same principle as RAPDs, but employ primers with sequences that are complementary to naturally occurring genomic DNA sequences (Rademaker \& de Bruijn, 1997; Thies et al., 2001). The resolving power of these methods vary greatly within and among certain groups of organisms and may also be dependent on the primers used (Thies et al., 2001; Vinuesa et al., 2005a; De Lajudie et al., 1998b) allowing for the identification of strains of a single species (Vinuesa et al., 2005a). Overall, however, these methods are extremely powerful for among-strain discrimination (Schneider, \& de Bruijn, 1996; Rademaker \& de Bruijn, 1997; Thies et al., 2001; Vinuesa et al., 2005a; Martens et al., 2008) and their major advantage lies in the fact that they take into account plasmids, nonessential genes and DNA. 


\section{(c) Amplified fragment length polymorphism (AFLP)}

Amplified fragment length polymorphism (AFLP) is a PCR-based fingerprinting method that was developed by Vos et al. (1995). It involves amplification of restriction fragments from total genomic DNA digests, after which fragments are separated using denaturing polyacrylamide gel electrophoresis. Compared to other marker technologies such as RAPD and RFLP, AFLP

provides greatly enhanced performance reproducibility, resolution and time efficiency. This technique can be used for the identification of highly related bacterial strains. It is useful as a rapid screening technique for large collections of bacterial isolates. This method also yields results that are comparable to DNA-DNA hybridization (see below) (Willems et al., 2001).

\subsubsection{Analyses of whole genomes using non-fingerprinting methods}

\section{(a) DNA-DNA hybridization}

DNA-DNA hybridization forms the basis for the determination of genera and species of bacteria. It reflects the sequence similarity between two entire genomes (Wayne et al., 1987). The technique is based on the characteristic ability of DNA to hybridize, a process that depends on similarity of the two sets of nucleotide sequences. This allows for quantification of the degree of relatedness and is expressed as percent similarity or homology. Genetically closely related organisms will have more nucleotide sequences in common and therefore a higher degree of nucleotide binding will occur (Vandamme et al., 1996). Based on DNA-DNA hybridization profiles a group of strains with homology of $70 \%$ or more under optimal hybridization conditions belong to the same genetic species (Wayne et al., 1987).

The method has proved reliable and has been used by various researchers for rhizobial characterization (Mergaert et al., 2002; Wei et al., 2003; Turado et al., 2005; Van Berkum et al., 2006; Mantelin et al., 2006; Chen et al., 2006). The major drawback of this technique is that it is technically challenging, labour intensive, time-consuming, non uniform and the method varies with different laboratories. It also requires a large amount of superior quality DNA for the entire genome, which is not always easy to obtain (Willems et al., 2001). Another drawback is that the results obtained are not always clear-cut. Hybridization values of $50 \%$ or less are less informative and therefore DNA-DNA hybridizations are not suitable for the estimation of genetic distances between distantly related species (Owen \& Pitcher, 1983; Martens et al., 2008). 
(b) Whole genome sequencing

It is becoming increasingly possible to determine the nucleotide sequence information for the entire genome of a specific bacterium. The process usually involves random fragmentation of total genomic DNA followed by cloning of the resulting fragments and sequencing using vectorspecific primers and standard Sanger sequencing (Sanger et al., 1977) procedures. The recent introduction of commercial sequencing-by-synthesis instruments (e.g. Roche GS GLX and Ilumina Solexa) (Braslavsky et al., 2003; Margulies et al., 2005), largely negates the need for generating clone libraries for sequencing, although these technologies are associated with various important drawbacks (Bentley, 2006). Nevertheless, whole genome sequences are available for a number of important rhizobia (see the website http://www.genomesonline.org). Such genomic sequence data may not yet be directly applicable for classification purposes, but their analyses provide valuable information regarding the evolution of rhizobia.

\subsubsection{Analyses of specific genomic loci}

\section{(a) Amplified rDNA restriction analysis (ARDRA)}

Amplified rRNA restriction analysis (ARDRA) targets the genes encoding 16S or 23S rRNA or parts of both genes with or without the intergenic spacer (IGS) region (Willems et al., 2000). The specific region is amplified using universal primers located in the conserved regions and digested with one or more restriction enzymes. ARDRA is a rapid technique, less demanding than direct sequencing and can distinguish between closely related species (Vandamme et al., 1996). This method has been applied for the differentiation of many bacterial genera, including several species of rhizobia (e.g. Rhizobium galegae) (Terefework et al., 1998).

A detailed study by Gürtler \& Stanisich (1996), showed that the IGS region was composed of highly conserved blocks of sequences while others were more variable. As a result, ARDRA analyses targeting the IGS allow for a higher level of discrimination, particularly between closely related species and have been used for identification purposes of a number of rhizobia species (Gürtler \& Stanisich 1996; Willems et al., 2001). An IGS-based ARDRA analysis has also been demonstrated to be useful at the intraspecific level. For example, Laguerre et al., (1996) applied this method to differentiate the biovars of Rhizobium leguminosarum. 


\section{(b) RPO1 fingerprinting analyses}

The RPO1 primer targets nif genes and can be used to identify specific strains of rhizobia (Richardson et al., 1995; Thies et al., 2001). This primer corresponds to a conserved reiterated element of the Rhizobium leguminosarum biovar trifolii nifHDK promoter. A directed sequencespecific RPO1 primer of 20 nucleotides in length was designed to differentiate a diverse collection of $R$. meliloti, $R$. leguminosarum biovars and a wide range of rhizobial and bradyrhizobial strains (Richardson et al., 1995). The RPO1 primer is capable of generating amplification profiles at annealing temperatures as high as $65^{\circ} \mathrm{C}$, compared to other primers. The temperature stability of RPO1 resulted in a much wider applicability for differentiation of rhizobia (Richardson et al., 1995; Botha et al., 2002; Botha et al., 2004) compared to methods using small 10-20-mer oligonucleotides (e.g. RAPDs) that would not anneal at these high temperatures (Richardson et al., 1995).

\section{(c) DNA sequence information for ribosomal RNA genes and regions}

Most previous studies used comparison of sequences of $16 \mathrm{~S}$ rRNA genes to differentiate between species of rhizobia (Young et al., 1991; Jarvis et al., 1992; Eardly et al., 1992; Yanagi \& Yamasato, 1993; Willems \& Collins, 1993; Laguerre et al., 1994). This is largely due to the fact that rRNA molecules are functionally stable, since they play an important role in protein synthesis. They consist of both conserved regions, for comparing distantly related organisms and variable regions used for comparing or grouping more closely related organisms (Woese, 1987). These features make RNA gene (5S, 16S, and 23S) and intergenic sequences very good choices to compare organisms and to infer phylogenies. Partial and complete 16S rRNA sequence analysis is a rapid tool for characterization of strains, though the technique lacks the ability to distinguish very closely related individuals (Sullivan et al., 1996; Willems et al., 2001; Martens et al., 2008), but has high resolving power for measuring the degree of relatedness between organisms above species level. Those organisms with genomic similarity of $70 \%$, when assessed by DNA-DNA homology, will share more than 97\% 16S rRNA gene sequence similarity (Stackebrandt \& Goebel, 1994).

Phylogenetic analysis using the sequence of the conserved 16S rRNA gene is a common and widely applied tool when classifying bacteria. Both the $16 \mathrm{~S}$ rRNA gene and to a lesser extent the 23S rRNA gene, have been used to identify new species with limited variability between strains of bacterial species (Barry et al., 1991). In most cases, however, this gene provides very little resolution at the interspecies level (Stackebrant \& Goebel, 1994; Willems et al., 2001; 
Vinuesa et al., 2005a). Also, several studies have demonstrated the presence of chimeric $16 \mathrm{~S}$ rRNA sequences in certain rhizobia and evidence for transfer of complete $16 \mathrm{~S}$ sequences between unrelated rhizobia (Eardly et al., 1996; Sullivan et al., 1996; Van Berkum et al., 2003). Problems associated with this gene are also encountered when it is used as the only phylogenetic marker as its phylogenies are extremely sensitive to unequal evolutionary rates (among taxa and among sites in the gene) and phylogenetic tree building artefacts (Nichols, 2001). Therefore, many researchers support the notion of basing taxonomic decisions on phylogenetic analyses of sequences for multiple loci (Nichols, 2001; Stepkowski et al., 2003; van Berkum et al., 2003; Vinuesa et al., 2005a).

\section{(d) DNA sequence information for other housekeeping gene sequences}

A range of protein-coding housekeeping genes are used for studying the taxonomy of rhizobia. In addition to the ribosomal genes, other 'housekeeping' genes with basic cell functions such as genes encoding recombinase A $(r e c A)$, glutamine synthetase isoform II $(g \ln I I), 70$ kilodalton heat shock protein $(\operatorname{dnaK})$, ATPase subunit D (atpD), etc. have been used to characterize rhizobia (Stepkowski et al., 2005; Vinuesa et al., 2005a), as they have profound usefulness in inferring phylogenetic relationships (Thies et al., 2001; Parker et al., 2002; Vinuesa et al., 2005a; Martens et al., 2008). In most cases these are applied for Multilocus sequence analysis (MLSA), an alternative method to 16S rRNA sequence analysis and DNA-DNA hybridization. The information from the comparison of multiple genes also gives a consistent overview of interorganism relationships at a wide range of taxonomic levels (Gervers et al., 2005). MLSA has been shown to be superior to $16 \mathrm{~S}$ rRNA gene sequencing analysis for Sinorhizobium species discrimination (Martens et al., 2007).

\section{(e) DNA sequence information for symbiotic (sym) loci}

Rhizobia encode their symbiotic properties at the sym loci, which forms part of the accessory genome (Parker et al., 2002). These include the nodulation genes (nod, nol and noe) and nitrogen-fixation (nif, fix) genes which confer the ability to nodulate and fix nitrogen in symbiosis with legume hosts, respectively. Sequencing of symbiotic genes has been used for the identification and association of rhizobia and it has mostly been found to reflect geographic or host relationships among isolates (Haukka et al., 1998; Parker et al., 2002). The comparison of these sequences to those of housekeeping or core genes therefore highlights the involvement of HGT in the evolution of the sym loci (Moulin et al., 2001; Moulin et al., 2004; Sawada et al., 2003, Martens et al., 2008). Phylogenetic trees inferred from these sym genes are usually 
strongly incongruent with the rhizobial housekeeping loci, typically revealing relationships that are host- and geographic-based rather than based on taxonomic status (Dobert et al., 1994; Parker et al., 2002; Stepkowski et al., 2005; Vinuesa et al., 2005a). As a result the sequences encoding nif and nod genes have been used to conclusively show that various regional rhizobial populations have been effectively isolated during evolutionary time, and has resulted in the appearance of locally adapted ecotypes or biovarieties (e.g. Parker et al., 2002; Sawada et al., 2003; Stepkowski et al., 2005; Vinuesa et al., 2005a; Steenkamp et al., 2008).

\subsubsection{Polyphasic approach}

Most, if not all, current rhizobial classification procedures involve a polyphasic approach whereby different data and information on groups of isolates are integrated to generate a consensus type of taxonomy (Vandamme et al., 1996; Reis et al., 2004; Vinuesa et al., 2005b). Polyphasic taxonomy results in reliable resolution of relationship among microorganisms (Vandamme et al., 1996; Martens et al., 2008). Vinuesa et al., (2005b) determine the adequacy of the combined phylogenetic and population genetic methods for the description of bacterial species, more especially Bradyrhizobium species. This approach gives scientifically sound information. Woese (1987) also stressed that a small number of characters is unreliable for defining taxa. The move to a polyphasic approach (using several characters) was made to ensure that only valid new species or genera are created. For the description of a new taxa all genotypic (DNA-DNA hybridization, etc.), phenotypic (substrate utilization, etc.) and phylogenetic (rRNA gene sequence analysis, etc.) information should therefore be combined (Stackebrandt \& Goebel, 1994; Martens et al., 2008). As a result rhizobial classifications should be based on phylogenetic and phenotypic data relating to the symbiotic, cultural, and morphological properties of the bacteria. Descriptions should also be based on data from a large number of strains from different geographical regions and hosts (Graham et al., 1991). In my MSc. study, a polyphasic approach was used to study the diversity of the rhizobia associated with the root nodules of Lebeckia species by using symbiotic characters (i.e. nodulation tests and nodule morphology), RPO1 and CRL7 fingerprint analyses and 16S rRNA-based phylogenetic analysis. 


\subsection{REFERENCES}

Achouak, W., Christen, R., Barakat, M., Martel, M. H., \& Heulin, T. 1999. Burkholderia caribensis sp. nov., an exopolysaccharide-producing bacterium isolated from vertisol microaggregates in Martinique. Int. J. Syst. Bacteriol. 49: 797-794.

Allen, O. N. \& Allen, E. K. 1981. The Leguminosae: A source book of characteristics, uses and nodulation. MacMillan, London.

Amarger, N., Macheret, V., \& Laguerre, G. 1997. Rhizobium gallicum sp. nov. and Rhizobium giardinii sp. nov. from Phaseolus vulgaris nodules. Int. J. Syst. Bacteriol. 47: 996-1006.

Angiosperm Phylogeny Group, 2003. An update of the Angiosperm Phylogeny Group classification for the orders and families of the flowering plants: APGII. Bot. J. Linn. Soc. 141: 399-436.

Baldani, V. L. D., Baldani, J. I., \& Döbereiner, J. 2000. Inoculation of rice plants with the endophytic diazotrophs Herbaspirillum seropedicae and Burkholderia spp. Biol. Fertil. Soils. 30: 485-491.

Barrett, C. F., \& Parker, M. A. 2006. Coexistence of Burkholderia, Cupriavidus and Rhizobium sp. nodule bacteria on two Mimosa spp. in Costa Rica. Appl. Environ. Microbiol. 72: 1198-1206.

Barry, T., Colleran, G., Glennon, M., Dunican, L. K., \& Gannon, F. 1991. The 16S/23S ribosomal spacer region as a target for DNA probes to identify eubacteria. PCR Methods Appl. 1: $51-56$.

Bentham, G. 1844. Enumeration of Leguminosae indigenous to southern Asia and Central and Southern Africa. Hook. Lond. J. Bot. 3: 338-365.

Bentley, D. R. 2006. Whole-genome re-sequencing. Curr. Opin. Genet. Dev. 16: 545-552. 
Bergersen, F. J. 1980. Methods for evaluating biological nitrogen-fixation. John Wiley and Sons, New York. Pp 337-367.

Bloem, J. F., Botha, W. J., Law, I. J., \& Steyn, P. L. 2002. Colony variation in Sinorhizobium meliloti inoculant strain U 45. Microbiol. Res. 157: 283-292.

Boatwright, J. S, van Wyk, B. - E., \& Tilney, P. M. 2007. Systematic studies in the genus Lebeckia and related genera: A revision of Lebeckia section Stiza (Crotalarieae, Fabaceae). S. Afr. J. Bot. 73: 280

Bohlool, B. B., Ladha, J. K., Garrity, D. P., \& George, T. 1992. Biological nitrogen-fixation for sustainable agriculture: a perspective. Plant Soil. 141: 1-11.

Boivin, C., \& Giraud, E. 1999. Molecular symbiotic characterization of rhizobia: toward a polyphasic approach using Nod factors and nod genes. In: Highlights of nitrogen-fixation research. Martinez-Romero, E., Hernández, G. (Eds). New York: Kluwer Academic/Plenum Publishers. Pp 295-299.

Botha, W. J., Bloem, J. F. \& Law, I. J. 2002. Bradyrhizobium sp. (Lupinus) in the winter rainfall region of South Africa. Biol. Fertil. Soils. 36: 335-343.

Botha, W. J., Jaftha, J. B., Bloem, J. F, Habig, J. H. \& Law, I. J. 2004. Effect of soil bradyrhizobia on the success of soybean inoculant strain CB 1809. Microbiol. Res. 159 : 219231.

Braslavsky, I., Hebert, B., Kartalov, E., \& Quake, S. 2003. Sequence information can be obtained from single DNA molecules. Proc. Natl. Acad. Sci. USA. 100: 3960-3964.

Brenner, D. J., Krieg, N. R., \& Staley, J. T. 2005. The Alpha-, Beta-, Delta-, and Epsilonproteobacteria. In: Garrity, G. M. (Ed). Bergey's Manual of systematic bacteriology, Second edition. Springer-Verlag, New York, Heildelberg. http://www.cme.msu.edu/Bergeys/ 
Brockwell, J., Bottomley, P. J., \& Thies, J. E. 1995. Manipulation of rhizobia microflora for improving legume productivity and soil fertility: A critical assessment. Plant Soil. 174: 143180.

Caballero-Mellado, J., Martinez-Aguilar, L., Paredes-Valdez, G., \& Estrada-de los Santos, P. 2004. Burkholderia unamae sp. nov., an N2-fixing rhizospheric and endophytic species. Int. J. Syst. Evol. Microbiol. 54: 1165-1172.

Carlson, R.W., Sanjuan, J., Bhat, U. R., Glushka, J., Spaink, H. P., Wijfjes, A. H., van Brussel, A. A., Stokkermans, T. J., Peters, N. K., \& Stacey, G. 1993. The structures and biological activities of the lipooligosaccharide nodulation signals produced by type I and type II strains of Bradyrhizobium japonicum. J. Biol. Chem. 268: 8372-18381.

Chen, W. X., Yan, G. H., \& Li, J. L. 1988. Numerical taxonomic study of fast-growing soybean rhizobia and a proposal that Rhizobium fredii be assigned to Sinorhizobium gen. nov. Int. J. Syst. Bacteriol. 38: 392-397.

Chen, W. X., Li, Y. L., Wang, E. T., Yaun, H. L., \& Li, J. L. 1991. Rhizobium huakii sp. nov. isolated from the root nodules of Astragalus sinicus. Inst. J. Syst. Bacteriol. 41: 275-280.

Chen, W. X., Wang, E. T., Li, Y. L., \& Chen, X. 1995. Characteristics of Rhizobium tianshanense sp. nov., a moderately and slowly growing root nodule bacterium isolated from arid saline environment in Xinjiang, People's Republic of China. Inst. J. Syst. Bacteriol. 45: 153159.

Chen, W. X., Wang, E. T., Li, Y., Tan, Z. Y., \& Gao, J. L. 1997. Rhizobium hainanense sp. nov., isolated from tropical legumes. Int. J. Syst. Bacteriol. 47: 870-873.

Chen, W. M., Laevens, S., Lee, T. M., Coenye, T., De vos, P., Mergeay, M. \& Vandamme, P. 2001. Ralstonia taiwanensis sp. nov., isolated from root nodules of Mimosa species and sputum of a cystic fibrosis patient. Int. J. Syst. Microbiol. 51: 1729-1735. 
Chen, W. M., Moulin, L., Bontemps, C., Vandamme, G. B., \& Masson, C. B. 2003a. Legume symbiotic nitrogen-fixation by $\beta$-Proteobacteria is widespread in nature. J. Bacteriol. 185 : 7266-7272.

Chen, W. M., James, E. K., Prescott, A. R., Kieraus, M. \& Sprent, J. I. 2003b. Nodulation of Mimosa spp. by $\beta$-proteobacterium Ralstonia taiwanensis. Mol. Plant Microbe Inter. 16: 10511061.

Chen, W. M., James, E. K., Coenye, T., Chou, J. H., Barrios, E., de Faria, S. M., Elliott, G. N., Sheu, S.Y., Sprent, J. I., \& Vandamme, P. 2006. Burkholderia mimosarum sp. nov., isolated from root nodules of Mimosa spp. from Taiwan and South America. Int. J. Syst. Evol. Microbiol. 56: 1847 - 1851.

Chen, W. M., de Faria, S. M., James, E.K., Elliott, G. N., Lin, K. Y., Chou, J. H., Sheu, S. Y., Cnockaert, M., Sprent, J. I., \& Vandamme, P. 2007. Burkholderia nodosa sp. nov., isolated from root nodules of the woody Brazilian legumes Mimosa bimucronata and Mimosa scabrella. Int. J. Syst. Evol. Microbiol. 57: 1055-1059.

Coenye, T., Laevens, S., Willems, A., Ohlen, M., Hannant, W., Govan, J. R. W. Gillis, M., Falsen, E., \& Vandamme, P. 2001. Burkholderia fungorum sp. nov. and Burkholderia caledonica sp. nov., two new species isolated from the environment, animals and human clinical samples. Int. J. Syst. Evol. Microbiol. 51: 1099-1107.

Coenye, T. \& Vandamme, P. 2004. Diversity and significance of Burkholderia species occupying diverse ecological niches. Mini Rev. Environ. Microbiol. 5: 719-729.

Corby, H.D.L., Polhill, R. M., \& Sprent, J. I. 1983. Taxonomy. In: Broughton, W. J. (Ed). Nitrogen-fixation, Legumes, vol. 3. Clarendon Press, Oxford. Pp 1-35.

Crisp M. D., Gilmore, S., \& Van Wyk, B. E. 2000. Molecular phylogeny of the genistoid tribes of Papilionoid legumes. In: Herendeen, P. S. \& Bruneau, A (Eds.). Advances in legume systematics, part 9. Royal Botanic Gardens, Kew. Pp 249-276. 
Cronk, Q., Ojeda, I., \& Pennington, R. T. 2006. Legume comparative genomics: progress in phylogenetics and phylogenomics. Curr. Opin. Plant Biol. 2: 99-103.

Dean, D. R., Bolin, J. T., \& Zheng, L. 1993. Nitrogenase Metalloclusters: Structures, Organization and synthesis. J. Bacteriol. 175: 6737-6744.

De Lajudie, P., Willems, A., Pot B., Dewettinck, D., Maestrojuan, G., Neyru, M., Collins, M. D., Dreyfus, B., Kersters, K., \& Gillis, M. 1994. Polyphasic taxonomy of rhizobia: Emendation of the genus Sinorhizobium and description of Sinorhizobium meliloti comb. nov., Sinorhizobium saheli sp., nov., and Sinorhizobium teranga sp. nov. Int. J. Syst. Bacteriol. 44: 715-733.

De Lajudie, P., Willems, A., Nick, G., Moreira, F., Molouba, F., Hoste, B., Torck, U., Neyra, M., Collins, M. D., Lindstrom, K., Dreyfus, B., \& Gillis, M. 1998a. Characterization of tropical tree rhizobia and description of Mesorhizobium plurifarium sp. nov. Int. J. Syst. Bacteriol. 48: 369- 382.

De Lajudie, P., Laurent-Fulele, E., Willems, A., Torck, U., Coopman, R., Collins, M. D., Dreyfus, B., Kersters, K., \& Gillis, M. 1998b. Allorhizobium undicola gen. nov., nitrogenfixing bacteria that efficiently nodulate Neptunia natans in Senegal. Int. J. Syst. Bacteriol. 48: 1277-1283.

De Souza Moreira, F. M., Cruz, L., de Faria, S. M., Marsh, T., Martinez-Romero, E., de Oliveira Pedrosa, F., Pitard, R. M., \& Young, J. P. W. 2006. Azorhizobium doebereinerae sp. nov. Microsymbionts of Sesbania virgata (Caz.) Pers. Syst. Appl. Microbiol. 29: 197-206.

Dobert, R. C., Breil, B. T., \& Triplet, E. W. 1994. DNA sequence of the common nodulation genes of Bradyrhizobium elkanii and their phylogenetic relationships to those of other nodulating bacteria. Mol. Plant Microbiol. Interact. 7: 564-572.

Doyle, J. J., Harrison, S. P., Mytton, L. R., Dye, M., Cresswell, A., Skot, L., \& Breeching, J. R. 1993. Phylogenetic grouping and identification of Rhizobium isolates on the basis of random amplified polymorphic DNA profiles. Can. J. Microbiol. 39: 665-673. 
Doyle, J. J. 1998. Phylogenetic perspectives on nodulation: evolving views of plants and symbiotic bacteria. Trends Plant Sci. 3: 473-479.

Doyle, J. J., Chappill, J. A., Bailey, C. D., \& Kjita, T. 2000. Towards a comprehensive phylogeny of legumes: evidence from $r b c L$ sequences and non-molecular data. In: Herendeen, P. S. \& Bruneau, A. (Eds). Advances in legume systematics, part 9. Royal Botanic Gardens, Kew. Pp 1-20.

Doyle, J. J., \& Luckow, M. A. 2003. The rest of the Iceberg: Legume diversity and evolution in a phylogenetic context. Plant Physiol. 131: 900-910.

Dreyfus, B., Garcia, J. L., \& Gillis, M. 1988. Characterization of Azorhizobium caulinodans gen. nov. sp. nov., a stem-nodulating nitrogen-fixing bacterium isolated from Sesbania rastrata. Int. J. Syst. Bacteriol. 38: 89-98.

Eardly, B. D., Materon, L. A., Smith, N. H., Johnson, D. A., Rumbaugh, M. B., \& Selander, R. K. 1990. Genetic structure and natural populations of the nitrogen-fixing bacterium Rhizobium meliloti. Appl. Environ. Microbiol. 56: 187-194.

Eardly, B. D., Young, J. P. W., \& Selander, R. K. 1992. Phylogenetic position of Rhizobium sp. Strain Or 191, a symbiont of both Medicago savita and Phaseolus vulgaris, based on partial sequences of the 16S rRNA and nifH genes. Appl. Environ. Microbiol. 58 : 1809-1815.

Eastrada-de los Santos, P., Bustillos-Critales, R., \& Caballero-Mellado, J. 2001. Burkholderia, a genus rich in plant associated nitrogen fixers with wide environmental and geographic distribution. Appl. Environ. Microbiol 67: 2790-2798.

Elliott, G. N., Chen, W. M., Bontemps, C., Chou, J. H., Young, J. P. W. Sprent, J. I., \& James, E. K. 2007. Nodulation of Cyclopia spp.(Leguminosae, Papilionoideae) by Burkholderia tuberum. Ann. Bot. 100: 1403-1411.

Euzéby, J.P. 1997. List of bacterial names with standing in nomenclature: a folder available on the internet. Int. J. Syst. Bacteriol., 47: 590-592. (List of Prokaryotic names with Standing in Nomenclature. Last full update: March 05, 2008. URL: http://www.bacterio.net) 
Fred, E. B.; Baldwin, I. L. \& McCoy, E. 1932. Root nodule bacteria and leguminous plants. University of Wisconsin studies in Science.

Gao, J. L., Turner, S. L., Kan, F. L., Wang, E. T. Tan, Z. Y., Qiu, Y. H., Gu, J., Terefework, Z., Young, J. P. W. Lindstrom, K., \& Chen, W. X. 2004. Mesorhizobium septrionale sp. nov. and Mesorhizobium temperatum sp. nov. isolated from Atragalus adsurgens growing in the northern regions of China. Int. J. Syst. Evol. Microbiol. 54: 2003-2012.

Garcia, M. C., Marina, M. L., Laborda, F., \& Torre, M. 1998. Chemical characterization of commercial soybean products. Food Chem. 62: 325-331.

Garcia-Fraile, P., Rivas, R., Willems, A., Peix, A., Martens, M., Martinez-Molina, M., Mateos, P. F., \& Velazquez, E. 2007. Rhizobium cellulosilyticum sp. nov., isolated from sawdust of Populus alba. Int. J. Syst. Evol. Microbiol. 57: 844-848.

Germishuizen, G., \& Meyer, N. L. 2003. Plants of Southern Africa, an annotated checklist. Strelitzia 14. National Botanical Institute, Pretoria. Pp 523-524.

Gervers, D., Cohan, F. M., Lawrence, J. G., Spratt, B. G. Coenye, t., Feil, E. T., Stackebrandt, E., Van der Peer, Y., Vandamme, P., Thompson, F. L., \& Swings, J. 2005. Re-evaluating prokaryotic species. Nat. Rev. Microbiol. 3:733-739.

Ghosh, W., \& Roy, P. 2006. Mesorhizobium thiogangeticum sp. nov., a novel sulfur-oxidizing chemolithoautotroph from rhizosphere soil of an Indian tropical leguminous plant. Int. J. Syst. Evol. Microbiol. 56: 91-97.

Giller, K. E. 2001. Nitrogen-fixation in Tropical cropping systems. CAB international. Wallingford.

Goris, J., De Vos, P., Caballero-Mellado, J., Park, J., Falsen, E., Quensen, J. F., Tiedje, J. M., \& Vandamme, P. 2004. Classification of the biphenyl- and polychlorinated biphenyl-degrading strain $\mathrm{LB}_{400^{\mathrm{T}}}$ and relatives as Burkholderia xenovorans sp. nov. Int. J. Syst. Evol. Microbiol. 54: 1677-1681. 
Gürtler, V., \& Stanisich, V. A. 1996. New approaches to typing and identification of bacteria using the 16S-13S rDNA spacer region. Microbiol. 142: 3-16.

Graham, P. H., Sadowsky, M. J., Keiser, H. H., Barnet Y. M., Bradley, R. S., Cooper, J. E., De Ley D. J. Jarvis B. D. W., Roslycky, E. B.,Strijdom, B. W., \& Young, J. P.W. 1991. Proposed minimal standards for the description of new genera and species of root- and stem-nodulating bacteria. Int. J. Syst. Bacteriol. 41: 582-587.

Graham, P. H., \&Vance, C. P. 2003. Legumes: Importance and constraints to greater use. Plant Physiol. 131: 872-877.

Graham, P. H. 1998. Biological dinitrogen fixation: Symbiotic. In: Sylvia, D. M., Fuhrmann, J. J., Hartel, P. G. \& Zuberer, D. A. (Eds). Principles and applications of soil microbiology. Second Edition. Prentice Hall, New Jersey. Pp 405-432.

Granli, T., \& Bøckman, O. C. 1994. Nitrous oxide from agriculture. Norw. J. Agric. Sci. Suppl. 12: 7-128.

Green, P. 1992. The genus Methylobacterium. In: Balows, A. , Truper, H. G., Dworkin, M., Harder, W., \& Schleifer, K. H. (Eds). The prokaryotes, Second edition. Springer-Verlag, New York. Pp 2342-2349.

Haukka, K., Lindstrom, K., \& Young, J. P. W. 1998. Three phylogenetic groups of nodA and nifH genes in Sinorhizobium and Mesorhizobium isolates from leguminous trees growing in Africa and Latin America. Appl. Environ. Microbiol. 64: 419-426.

Hardy, R. W. F., \& Eaglesham, A. R. J. 1995. Ecology and agriculture applications of nitrogen-fixing systems: overview. In: Tikhonov, I. A., Provorov, N. A., Romanov, V. I. \& Newton, W. E. (Eds). Nitrogen-fixation: Fundamentals and applications. Kluwer Academic Publishers, Dordrecht. Pp 19-622.

Harvey, W. H. 1862. Leguminosae. In: Harvey, W. H. \& Sonder, O. W. (Eds). Flora Capensis, volume 2. Hodges, Smith, \& Co., Dublin. Pp 83-86. 
Heichel, G. H., \& Vance, C. P. 1983. Physiology and morphology of perennial legumes. In: Broughton, W. J. (Ed). Nitrogen-fixation, Legumes, vol. 3. Clarendon Press, Oxford, Pp 99133.

Herendeen, P. S., Bruneau, A., \& Lewis, G. P. 2003. Phylogenetic relationships in caesalpinioid legumes: a preliminary analysis based on morphological and molecular data. In: Klitgaard, B.B., Bruneau, A. (Eds). Advances in legume systematics, part 10. Royal Botanic Gardens, Kew, Pp 37-62.

Herendeen, P. S., Crepet, W. L., \& Dilcher, D. L. 1992. The fossil history of the Leguminosae: phylogenetic and biogeographic implications. In: Herendeen, P. S., Dilcher, D. L. (Eds). Advances in Legume systematics, part 4. The fossil record. Royal Botanic Gardens, Kew. Pp 303-316

Hirsch, P. \& Müller M. 1985. Blastobacter aggregatus sp. nov., Blastobacter capsulatus sp. nov., and Blastobacter denitrificans sp. nov., new budding bacteria from freshwater habitats. Syst. Appl. Microbiol. 6: 281-286.

Holland, M. A. 1997. Methylobacterium and plants. Recent Res. Dev. Plant Physiol. 1: 207212.

Hollis, A. B., Kloos, W. E., \& Elkan, G. H. 1981. DNA-DNA hybridization studies of Rhizobium japonicum and related Rhizobiaceae. J. Gen. Microbiol. 123: 215-222.

Holmes, B. \& Roberts, P. 1981. The classification, identification and nomenclature of Agrobacteria. J. Appl. Bacteriol. 50: 443-467.

Holmes, B., Popoff, M., Kiredjian, M., \& Kersters, K. 1988. Ochrobactrum anthropi gen. nov., sp. nov. from human clinical specimen and previously known as group Vd. Int. J. Syst. Bacteriol. 38: 406-416.

Hungria, M., \& Stacey, G. 1997. Molecular signals exchanged between host plants and rhizobia: Basic aspects and potential application in agriculture. Soil Biol. Biochem. 29:819-830. 
Jaftha, J. B., Strijdom, B. W., \& Steyn, P. L. 2002. Characterization of pigmented Methylotrophic bacteria which nodulate Lotononis bainesii. Syst. Appl. Microbiol. 25: 440-449.

Jarvis, B. D. W., Downer, H. L., \& Young, J. P. W. 1992. Phylogeny of fast-growing soybeannodulating rhizobia supports synonymy of Sinorhizobium and Rhizobium assignment to Rhizobium fredii. Int. J. Syst. Bacteriol. 42: 93-96.

Jarvis, B. D. W., van Berkum, P., Chen, W. X., Nour, S. M., Fernandez, M. P., Cleyet-Marel, J. C., \& Gillis, M. 1997. Transfer of Rhizobium loti, Rhizobium haukii, Rhizobium ciceri, Rhizobium medittereneum and Rhizobium tianshanense to Mesorhizobium gen. Nov. Int. J. Syst. Bacteriol. 47: 895-898.

Jordan, D. C. 1984 . Family III Rhizobiaceae Conn 1938. In: Krieg, N.R. \& Holt, J. G. (Eds) Bergey's manual of systematic bacteriology. William and Wilkins, Baltimore. Pp 234-243.

Jourand, P., Giraud, E., Bena, G., Sy, A., Willems, A., Gillis, M., Dreyfus, B., \& de Lajudie P. 2004. Methylobacterium nodulans sp. nov., for a group of aerobic, facultative methylotrophic, legume root-nodule-forming and nitrogen-fixing bacteria. Int. J. Syst Evol. Microbiol. 54: 22692273.

Kajita, T., Ohashi, H., Tateishi, Y., Bailey, C., \& Doyle, J. J. 2001. rbcL and legume phylogeny, with particular reference to Phaseoleae, Millettieae, and allies. Syst. Bot. 26(3): 515536.

Kneip, A., Simar, L., \& Wilson, P. W. 2007. Asymptotics and consistent bootstraps for DEA estimators in non-parametric frontier Models. J. Econ. Lit. 1-40

Kuykendall, L. D., Saxena, B., Devine, T. E., \& Udell, S. E. 1992. Genetic diversity in Bradyrhizobium japonicum Jordan 1982 and a proposal for Bradyrhizobium elkanii sp. nov. Can. J. Microbiol. 38: 501-505.

Kwon, S. W., Park, J. Y., Kim, J. S., Kang, J. W., Cho, Y. H. Lim, C. K., Parker, M. A., \& Lee, G. B. 2005. Phylogenetic analysis of the genera Bradyrhizobium, Mesorhizobium, Rhizobium 
and Sinorhizobium on the basis of 16S rRNA gene and internally transcribed spacer region sequences. Int. J. Syst. Evol. Microbiol. 55: 263-270.

Laguerre, G., Allard, M. R., Revoy, F., \& Amarger, N. 1994. Rapid identification of rhizobia by restriction fragment length polymorphism analysis of PCR-amplified 16S rRNA genes. Appl. Environ. Microbiol. 60: 56-63.

Laguerre, G., Mavingui, P., Allard, M. R., Charnay, M. P., Louvrier, P., Mazurier, S. I., Rigottier-Gois, L., \& Amarger, N. 1996. Typing of rhizobia by PCR DNA Fingerprinting and PCR-Restriction Fragment Length Polymorphism analysis of chromosomal and symbiotic gene regions: Application to Rhizobium leguminosarum and its biovars. Appl. Environ. Microbiol. 62: 2029-2036.

Lavin, M., Herendeen, P. S., \& Wojciechowski, M. F. Evolutionary rates analysis of Leguminosae implicates a rapid diversification of lineages during the tertiary. Syst. Biol. 54: 574-594.

Law, I. J., Botha, W. J., Majaule, U. C., \& Phalane, F. L. 2007. Symbiotic and genomic diversity of "Cowpea" bradyrhizobia from soils in Botswana and South Africa. Biol. Fertil. Soils. 43 : 653-663.

Le Roux, P. M., Kotze, G. P., \& Glen, H. F. 1994. Bossieveld. Grazing plants of the Karoo and Karoo-like areas. Bulletin 428. Department of Agriculture, Pretoria.

Le Roux, J. J. 2003. The diversity of root nodule bacteria associated with indigenous Lotononis spp. as determined by sodium dodecyl-sulphate polyacrylamide gel electrophosis and 16S rDNA sequencing. MSc. thesis. University of Pretoria, Pretoria, Republic of South Africa.

Le Roux, M. M., \& van Wyk, B. E. 2007. A revision of Lebeckia sect. Lebeckia: The L. semiarid group. S. Afr. J. Bot. 73: 118-130

Le Roux, M. M., van Wyk, B. E., \& van deer Bank, M. 2007. A taxonomic study of the type section of the genus Lebeckia Thumb. (Abaca, Crotalaria). S. Afr. J. Bot. 73: 297 
Le Roux, A., \& Schlep, T. 1997. Namaqualand: South Africa wildflower guide 1. Botanical Society of South Africa, Kirstenbosch.

Lopez-Lara, I. M., Blok-Tip, L., Quinto, C., Garcia, M. L., Stacey, G., Bloemberg, G. V., Lamers, G. E. M., Lugtenberg, B. J. J., Thomas-Oates, J. E., \& Spaink, H. P. 1996. NodZ of Bradyrhizobium extends the nodulation host range of Rhizobium by adding a fucosyl residue to nodulation signals. Mol. Microbiol. 21: 397-408.

Makkar, N. S., \& Casida, L. E. 1987. Cupriavidus necator gen. nov., sp. nov.: a nonobligate bacterial predator of bacteria in soil. Int. J. Syst. Bacteriol. 37: 323-326.

Manning, J., \& Goldblatt, P. 1996. West Coast: South African wildflower guide 7. Botanical Society of South Africa, Kirstenbosch.

Manning, J., \& Goldblatt, P. 1997. Nieuwoudtville Bokkeveld Plateau and Hantam West Coast: South African wildflower guide 9. Botanical Society of South Africa, Kirstenbosch.

Mantelin, S., Fisher-Le Saux, M., Zakhia, F., Bena, G., Bonneau, S., Jeder, H., de Lajudie, P., \& Cleyet-Marel, J. C. 2006. Emended description of the genus Phyllobacterium and description of four novel species associated with plant roots: Phyllobacterium bourgognense sp. nov., Phyllobacterium ifriqiyense sp. nov., Phyllobacterium leguminum sp. nov. and Phyllobacterium brassicacearum sp. nov. Int. J. Syst. Evol. Microbiol. 56: 827-839.

Margulies, M., Egholm, M., Altman, W. E., Attiya, S., Bader, J. S., Bemben, L. A., Berka, J., Braverman, M. S., Chen, Y. J., Chen, Z., Dewell, S. B., Du, L., Fierro, J. M., Gomes, X. V., Godwin, B. C., He, W., Helgesen, S., Ho, C. H., Irzyk, G.P., Jando, S.C., Alenquer, M. L. I., Jarvie, T.P., Jirage, K.B. Kim, J. B., Knight, J. R., Lanza, J. R. Leamon, J. H., Lefkowitz, S.M., Lei, M., Li, J., Lohman, K.L., Lu, H., Makhijani, V.B., McDade, K.E., Mckenna, M.P., Myers, E. W., Nickerson, E., Nobile, J. R., Plant, R., Puc, B.P., Ronan, M.T., Roth, G. T., Sarkis, G. J., Simons, J. F., Simpson, J. W., Srinivasan, M., Tartaro, K. R., Tomasz, A., Vogt, K.A., Volkmer, G. A., Wang, S. H., Wang, Y., Weiner, M. P., Yu, P., Begley, R. F., \& Rothberg, J. M. 2005. Genome sequencing in microfabricated high-density picolitre reactors. Nature. 437: 376-260. 
Martens, M., Delaere, M., Coopman, R., de Vos, P., Gillis, M., \& Willems, A. 2007. Multilocus sequence analysis of Ensifer and related taxa. Int. J. Syst. Evol. Microbiol. 57: 489503.

Martens, M., Dawyndt, P., Coopman, R., Gillis, M., de Vos, P., \& Willems, A. 2008. Advantages of multilocus sequence analysis for taxonomic studies: a case study using 10 housekeeping genes in the genus Ensifer (including former Sinorhizobium). Int. J. Syst. Evol. Microbiol. 58: 200-214.

Martinez-Romero, E., Segovia, L., Mercante, F. M., Franco, A. A., Graham, P., \& Pardo, M. A. 1991. Rhizobium tropici, a novel species nodulating Phaseolus vulgaris L. beans and Leucaena sp. trees. Int. Syst. Bacteriol. 41: 417-426.

Mathis, J. N., \& McMillin, D. E. 1996. Detection of genetic variation in Bradyrhizobium japonicum USDA 110 variants using DNA fingerprints generated with GC rich arbitrary PCR primers. Plant Soil. 186: 81-85.

McInroy, S. G., Campbell, C. D., Haukka, K. E., Odee, D. W., Sprent, J. I., Wang, W. J., Young, P. J. W., \& Sutherland, J. M. 1999. Characterization of rhizobia from African acacias and other tropical woody legumes using Biolog and 16S rRNA sequencing. Microbiol. Lett. 170: 111-117.

McKey, D. 1994. Legumes and nitrogen: the evolutionary ecology of a nitrogen-demanding lifestyle. In: Sprent, J. I. \& McKey, D. (Eds). Advances in legume systematics, part 5, The nitrogen factor. Royal Botanic Gardens, Kew. Pp 211-228.

Mergaert, J., Cnockaert, M. C., \& Swings, J. 2002. Phyllobacterium myrsinacearum (subjective synonym Phyllobacterium rubiacearum) emend. Int. J. Syst. Evol. Microbiol. 52: $1821-1823$.

Micheli, M. R., Bova, R., \& Ambrosio, E. D. 1996. Random amplified DNA assay. In: Micheli, M. R. \& Bova, R. (Eds). Fingerprinting methods based on arbitrarily primed PCR. Springer-Verlag, Berlin. Pp 47-65. 
Minchin, F. R., Minquez, M. I., Sheehy, J. E., Witty, J. F., \& Skøt, L. 1986. Relationships between nitrate and oxygen supply in symbiotic nitrogen-fixation by white clover. J. Exp. Bot. 37: 1103-1113.

Moulin, L., Munive, A., Dreyfus, B., \& Boivin-Masson, C. 2001. Nodulation of legumes by members of the $\beta$-subclass of Proteobacteria. Nature. 411: 948-950.

Moulin, L., Bena, G., Boivin-Masson, C., \& Stępkowski, T. 2004. Phylogenetic analysis of symbiotic nodulation genes supports vertical and lateral gene co-transfer within the Bradyrhizobium genus. Mol. Phylogen. Evol. 30: 720-732.

Nakagawa, Y., Sakane, T., \& Yokota, A. 1996. Transfer of "Pseudomonas riboflavina" (Foster 1944) a gram-negative, motile rod with long-chain 3-hydroxy fatty acids, to Devosia riboflavina gen. nov., sp. nov., nom. Rev. Int. J. Syst. Bacteriol. 46: 16-22.

Nichols, R. 2001. Gene trees and species trees are not the same. Trends Ecol. Evol. 16: 358364.

Nick, G., Jussila, M., Hoste, B., Niemi, M., Kaijalainene, S., de Lajudie, P., Gillis, M., de Bruin, F., \& Lindstrom, K. 1999a. Rhizobia isolated from the root nodules of tropical leguminous trees characterized using DNA-DNA dot blot hybridization and rep-PCR. Syst. Appl. Microbiol. 22: $287-299$.

Nick, G., de Lajudie, P., Eardly, B.D., Suomalainen, S., Paulin, L., Zahran, X., Gillis, M., de Bruin, F., \& Lindstrom, K. 1999b. Sinorhizobium arboris sp. nov. and Sinorhizobium kostiense sp. nov., isolated from leguminous trees in Sudan and Kenya. Int. J. Syst. Bacteriol. 49: 13591368.

Niemann, S., Puhler, A., Tichy, H. V., Simon, R., \& Selbitschka, W. 1997. Evaluation of the resolving power of three different DNA fingerprinting methods to discriminate among isolates of a natural Rhizobium meliloti population. Appl. Microbiol. 82: 447-484. 
Nour, S. M., Fernandez, M. P., Normand, P., \& Cleyet-Marel, J. C. 1994. Rhizobium ciceri sp. nov. consisting of strains nodulating chickpeas (Cicer arietinum L.). Int. J. Syst. Bacteriol. 44: $511-522$.

Nour, S. M., Cleyet-Marel, J., Normand, P., \& Fernandez, M. P. 1995. Genomic heterogeneity of strains nodulating Chickpeas (Cicer arietium L.) and description of Rhizobium mediterraneum sp. nov. Int. J. Syst. Bacteriol. 45: 640-648.

Owen, R. J., \& Pitcher, D. 1983. Current methods for determining DNA-base composition and levels of DNA hybridization. J. Appl Bacteriol. 55: R16-R16.

Parker, M. A., Lafay, B., Burdon, J. J., \& van Berkum, P. 2002. Conflicting phylogeographic patterns in rRNA and nifD indicate regionally restricted gene transfer in Bradyrhizobium. Microbiol. Res. 148: 2557-2565.

Payne, G. W., Vandamme, P., Morgan, S. H., Lipuma, J. J. Coenye, T., Weightman, A. J., Jones, T. H., \& Mahenthiralingam, E. 2005. Development of a recA gene-based identification approach for the entire Burkholderia genus. Appl. Environ. Microbiol. 71: 3917-3927.

Perin, L., Martinez-Aguilar, L., Castro-Gonzalez, R.,Estrada-de los Santos, P., CabellosAvelar,T., Guedes, H. V., Reis, V. M., \& Caballero-Mellado, J. 2006. Diazotrophic Burkholderia species associated with field-grown maize and sugar cane. Appl. Environ. Microbiol. 72: 3103-3110.

Perret, X., Staehelin, C., \& Broughton, W. J. 2000. Molecular basis of symbiotic promiscuity. Microbiol. Mol. Biol. Rev. 64: 180-201.

Quan, Z. X., Bae, H. S., Baek, J. H., Chen, W. F. Im, W. T., \& Lee, S. T. 2005. Rhizobium daejeonense sp. nov. isolated from a cyanide treatment bioreactor. Int. J. Syst. Evol. Microbiol. 55: 2543-2549.

Rademaker, J. L. W., \& de Bruijn, F. J. 1997. Characterization and classification of microbes by REP-PCR genomic fingerprinting and computer-assisted pattern analysis. http://www.msu.edu/user/debruijn/dna1-4.htm 
Reis, V. M., Estradade los Santos P., Tenorio-Salgado, S., Vogel, J., Stoffel, M., Guyon, S., Mavingui, P., Baldani, V. L. D. Schmid, M., Baldani, J. I., Balandreau, J., Hartmann, A., \& Caballero- Mellado, J. 2004. Burkholderia tropica sp. nov., a novel nitrogen-fixing, plant associated bacterium. Int. J. Syst. Evol. Microbiol. 54: 2155-2162.

Richardson, A. E., Viccars, L. A., Watson, J. M., \& Gibson, A. H. 1995. Differentiation of Rhizobium strains using the polymerase chain reaction with random and directed primers. Soil Biol. Biochem. 27: 515-524.

Rinaudo, G., Orenga, S., Fernandez, M. P., Meugnier, H., \& Bardin, R. 1991. DNA homologies among members of the genus Azorhizobium and other stem- and root-nodulating bacteria isolated from the tropical legume Sesbania rostrata. Int. J. Syst. Bacteriol. 41: 114-120.

Rivas, R., Willems, A., Subba-Rao, N. S., Mateos, P. F., Dazzo, F. B., Kroppenstedt, R. M., Martinez-Molina, E., Gillis, M., \& Velázquez, E. 2003. Description of Devosia neptunae sp. nov. that nodulates and fixes nitrogen in symbiosis with Neptunia natans, an aquatic legume from India. Syst. Appl. Micobiol. 26: 47-53.

Rivas, R., Willems, A., Palomo, J. L. Garcia-Benavides, P., Mateos, P. F., Martinez-Molina, E., Gillis, M., \& Velázquez, E. 2004. Bradyrhizobium betae sp. nov., isolated from roots of Beta vulgaris affected by tumour-like deformations. Int. J. Syst. Evol. Microbiol. 54: 1271-1275.

Rome, S., Fernandez, M. P., Brunel, B., Normand, P., \& Cleyet-Marel, J. C. 1996. Sinorhizobium medicae sp. nov., isolated from annual Medicago spp. Int. J. Syst. Bacteriol. 46: 972-980.

Sadowsky, M. J., \& Graham, P. H. 2004. Root nodule bacteria and legumes. Springer-Verlag, New York. Pp 1-36.

Sanger, F., Nicklen, S., \& Coulson, A. R. 1977. DNA sequencing with chain-terminating inhibitors. Proc. Natl Acad. Sci. USA. 74: 5463-5467. 
Sawada, H., Leki, H., Oyaizu, H., \& Matsumoto, S. 1993. Proposal for the rejection of Agrobacterium tumefaciense and Agrobacterium rhizogenes. Int. J. Syst. Bacteriol. 43: 694702.

Sawada, H., Kuykendall, L. D., \& Young, J. M. 2003. Changing concepts in the sytematics of bacterial nitrogen-fixing legume symbionts. J. Gen. Appl. Microbiol. 49: 155-179.

Schneider, M., \& de Bruijn, F. J. 1996. Rep-PCR mediated genomic fingerprinting of rhizobia and computer-assisted phylogenetic pattern analysis. World J. Microbiol. Biotech. 12: 163-174.

Scholla, M. H., \& Elkan, G. H. 1984. Rhizobium fredii sp. nov. a fast growing species that effectively nodulates soybeans. Int. J. Syst. Bacteriol. 34: 484-486.

Segovia, L., Young, J. P. W., \& Martinez-Romero, E. 1993. Reclassification of American Rhizobium leguminosarum biovar phaseoli type I strains as Rhizobium etli sp. nov. Int. J. Syst. Bacteriol. 43: 374-377.

Shearing, D., \& van Heering, K. 1994. Karoo South African wild flower: guide 6. Botanical Society of South Africa and National Botanical Institute, Kirstenbosch. Pp13-80.

Soltis, D. E., Soltis, P. S., Chase, M. W., Mort, M. E, Albach, D. C, Zanis, M., Savolainen, V., Hahn, W. H. Hoot, S. B. Fay, M. F., Axtell, M., Swensen, S. M., Prince, L. M., Kress, W. J., Nixon, K. C., \& Farris, J. S. 2000. Angiosperm phylogeny inferred from $18 \mathrm{~S}$ rDNA, rbcL and atpB sequences. Bot. J. Linn Soc. 133:381-461.

Somasegaran, P., \& Hoben, H. J. 1994. Hand book for Rhizobia. Springer-Verlag, Heidelberg.

Spaink, H. P., Kondorosi, A., \& Hooykaas, P. J. J. 1998. The Rhizobiaceae. Kluwer Academic Publishers, Dordrecht.

Sprent, J. I. 1995. Legume trees and shrubs in the tropics: Nitrogen-fixation in perspective. Soil Biol. Biochem. 27: 401-407. 
Sprent, J. I. 2001. Nodulation in legumes. Royal Botanic Gardens, London, Kew.

Sprent, J. I. 2007. Evolving ideas of legume evolution and diversity: a taxonomic perspective on the occurrence of nodulation. New Phytol. 174: 11-25.

Sprent, J. I., \& James, E. K. 2007. Legume evolution: Where do nodules and mycorrhizas fit in. Update on legume evolution. Plant Physiol. 144: 575-581.

Squartini, A., Struffi, P., Doring, H., Selenska-Pobell, S., Tola, E., Giacomini, A., Vendramin, E., Velazquez, E., Mateos, P.F. Martinez-Molina, E., Dazzo, F. B., Casella, S., \& Nuti, M. P. 2002. Rhizobium sullae sp. nov. (formerly 'Rhizobium hedysari'), the root-nodule microsymbiont of Hedysarum coronarium L. Int. J. Syst. Evol. Microbiol. 52: 1267-1276.

Stackebrandt, E., \& Goebel, B. M. 1994. Taxonomic note: a place for DNA-DNA reassociation and $16 \mathrm{~S}$ rRNA sequence analysis in the present species definition in bacteriology. Int. J. Syst. Bacteriol. 44: 847-849.

Steenkamp, E,T, Stępkowski, T., Przymusiak, A., Botha, W. J., \& Law, I. J. 2008. Cowpea and peanut in southern Africa are nodulated by diverse Bradyrhizobium strains harboring nodulation genes that belong to the large pantropical clade common in Africa. Mol. Phylogen. Evol. (Accepted for publication).

Stepkowski, T., Moulin, L., Krzyzanska, A., McInnes, A., Law I. J., \& Howieson, J. 2005. European origin of Bradyrhizobium populations infecting Lupins and Serradella in soils of Western Australia and South Africa. Appl. Environ. Microbiol. 71: 7041-7052.

Strijdom, B. W. 1998. South African studies on biological nitrogen-systems and the exploitation of the nodule bacterium-legume symbiosis. S. Afr. J. Sci. 94: 11-23.

Strijdom, B. W., \& Wassermann, V. D. 1984. Potential nitrogen contribution of legumes to agriculture. Plant Protection Research Institute. Review. Pp 175-177. 
Sullivan, J. T., Eadly, B., van Berkum, P., \& Ronson, C. W. 1996. Four unnamed species of nonsymbiotic rhizobia isolated from the rhizosphere of Lotus corniculatus. Appl. Environ. Microbiol. 62: 2818-2825.

Sy, A., Giraud, E., Jourand, P., Garcia, N., Willems, A., de Lajudie, P., Prin, Y., Neyra, M., Gillis, M., Boivin-Masson, C., \& Dreyfus, B. 2001. Methylotrophic Methylobacterium bacteria nodulate and fix nitrogen in symbiosis with legumes. J. Bacteriol. 183: 214-220.

Tan, Z. Y., Kan, F. L., Peng, G. X., Wang, E. T., Reinhold-Hurek, B., \& Chen, W. X. 2001. Rhizobim yanglingense sp. nov., isolated from arid and semi-arid regions in China. Int. J. Syst. Evol. Microbiol. 51: 909-914.

Taylor, D. W. 1990. Paleobiogeographic relationships of angiosperms from the Cretaceous and early Tertiary of the North American area. Bot. Rev. 56: 279-415.

Terefework, Z., Nick, G., Suomalainen, S., Paulin, L., \& Lindstrom, K. 1998. Phylogeny of Rhizobium galegae with respect to other rhizobia and agrobacteria. Int. J. Syst. Bacteriol. 48: 349-356.

Thies, J. E., Bohlool, B. B., \& Singleton, P. W. 1991. Subgroups of the Cowpea miscellany: Symbiotic Specificity within Bradyrhizobium spp. for Vigna unguiculata, Phaseolus lunatus, Arachis hypogaea and Macroptilium atropurpureum. Appl. Environ. Microbiol. 57: 1540-1545.

Thies, J. E., Holmes, E. M., \& Vachot, A. 2001. Application of molecular techniques to studies in Rhizobium ecology: A review. Aust. J. Exp. Agric. 41: 299-319.

Toledo, I., Lloret, L., \& Martinez-Romero, E. 2003. Sinorhizobium americanus sp. nov., a new Sinorhizobium species nodulating native Acacia spp. in Mexico. Syst. Appl. Microbiol. 26: 5464.

Trujillo, M. E., Willems, A., Abril, A., Planchuelo, A. M. Rivas, R., Ludena, D., Mateos, P. F., Martinez-Molina, E., \& Velazquez, E. 2005. Nodulation of Lupinus albus by strains of Ochrobactetrum lupine sp. nov. Appl. Environ. Microbiol. 71: 1318-1327. 
Valverde, A., Velazquez, E. Gutierrez,C. Cervantes, E., Ventosa, A., \& Iqual, J. M. 2003. Herbaspirillum lusitanum sp. nov., a novel nitrogen-fixing bacterium associated with root nodules of Phaseolus vulgaris. Int. J. Syst. Evol. Microbiol. 53 : 1979-1983.

Valverde, A., Velazquez, E. Fernandez-Santos, F., Vizcaino,N., Rivas, R., Mateos, P.F. Martinez-Molina, E., Igual, J.M., \& Willems, A. 2005. Phyllobacterium trifolii sp. nov., nodulating Trifolium and Lupinus in Spanish soils. Int. J. Syst. Evol. Microbiol. 55: 1985-1989.

Valverde, A., Igual, J. M., Peix, A., Cervantes, E., \& Velazquez, E. 2006. Rhizobium lusitanium sp. nov. a bacterium that nodulates Phaseolus vulgaris. Int. J. Syst. Evol. Microbiol. 56: 26312637.

Van Berkum, P., Beyene, D., Bao, G., Campbell, T. A., \& Eardly, B. D. 1998. Rhizobium mongolense sp. nov. is one of three rhizobial genotypes identified which nodulate and form nitrogen-fixing symbiosis with Medicago ruthenica [(L) Ledebour]. Int. J. Syst. Bacteriol. 48: $13-22$.

Van Berkum, P., \& Eardly, B. D. 1998. Molecular evolutionary systematics of the Rhizobiaceae. In: The Rhizobiaceae: Molecular Biology of model plant-associated bacteria. Pp 1-24. Spainck, H. P. (Ed). Dordrecht/ Boston/ London: Kluwer Academic Publishers.

Van Berkum, P., \& Eardly, B. D. 2002. The aquatic budding Bacterium Blastobacter denitrificans is a nitrogen-fixing symbiont of Aeschynomene indica. Appl. Environ. Microbiol. 68: 1132-1136.

Van Berkum, P., Terefework, Z., Paulin, L., Suomalainen, S., Lindstrőm, K. \& Eardly, B. E. 2003. Discordant phylogenies within the rrn loci of rhizobia. J. Bacteriol. 185: 2988-2998.

Van Berkum, P., Leibold, J. M., \& Eardly, B. D. 2006. Proposal for combining Bradyrhizobium spp.(Aeschynomene indica) with Blastorbacter denitrificans and to transfer Blastorbacter denitrificans (Hirsch and Muller, 1985) to the genus Bradyrhizobium as Bradyrhizobium denitrificans (comb. nov.). Syst. Appl. Microbiol. 29: 207-215. 
Van Breda, P. A. B., \& Barnard, S. A. 1991. 100 veld plants of the winter rainfall region. A guide to the use of veld plants for grazing. Bulletin No. 422. Department of Agricultural Development, Government press, Pretoria.

Vandamme, P., Pot, B., Gillis, M., Devos, P., Kersters, K., \& Swings, J. 1996. Polyphasic taxonomy, a consensus approach to bacterial systematics. Microbiol. Rev. 60: 407-438.

Vandamme, P., Goris, J., Chen, W. M., de Vos, P., \& Willems, A. 2002. Burkholderia tuberum sp. nov. and Burkholderia phymatum sp. nov., nodulate the roots of tropical legumes. Syst. Appl. Microbiol. 25: 507-512.

Vandamme, P., \& Coenye, T. 2004. Taxonomy of the genus Cupriavidis: a tale of lost and found. Int. J. Syst. Evol. Microbiol. 54: 2285-2289.

Van Heerden, F. R., Van Wyk, B. E. Viljoen, A. M., \& Steenkamp, P. A. 2003. Phenolic variation in wild populations of Aspalathus linearis (rooibos tea). Biochem. Syst. Ecol. 31: 885895.

Van Rhijn, R., \& Vanderleyden, J. 1995. The Rhizobium-legume symbiosis. Microbiol. Rev. 59: 124-142.

Van Rossum, D., Schuurmans, F. P., Gillis, M., Muyotcha, A., Van Verseveld, H. K., Stouthamer, A. H., \& Boogerd, F. 1995. Genetic and phenotypic analysis of Bradyrhizobium strains nodulating peanut (Arachis hypogaea L.) roots. Appl. Environ. Microbiol. 61:15991609.

Van Wyk, B. - E. 1991. A synopsis of the genus Lotononis (Fabaceae: Crotalarieae). Rustica Press Ltd, Wynberg.

Van Wyk, B. - E., \& Verdoorn, G. H. 1989a. Chemotaxonomic significance of alkaloids in the genus Lebeckia. Biochem. Syst. Ecol. 17: 225-229.

Van Wyk, B. - E., \& Verdoorn, G. H. 1989b. A chemotaxonomic survey of major alkaloids in Lotononis and Buchenroedera. Biochem. Syst. Ecol. 17: 385-389. 
Van Wyk, B. - E., \& Schutte, A. L. 1995. Phylogenetic relationships in the tribes Podalyrieae, Liparieae and Crotalarieae. In: Crisp, M. \& Doyle, J. J. (Eds). Advances in legume systematics, part 7, Phylogeny. Royal Botanic Gardens, Kew. Pp 283-308.

Van Wyk, B. - E., van Heerden, F., \& van Oudtshoorn, B. 2002. Poisonous plants of South Africa. Briza publications, Pretoria.

Vance, C. P. 2001. Symbiotic nitrogen-fixation and phosphorus acquisition. Plant nutrition in a world of declining renewable resources. Plant Physiol. 127: 390-397.

Vance, C. P., \& Graham, P. H. 1995. Nitrogen-fixation in agriculture: Application and perspectives. In: Tikhonov, I. A., Provorov, N. A., Romanov, V. I., \& Newton, W. E. (Eds). Nitrogen-fixation: fundamentals and applications. Kluwer, Dordrecht. Pp 77-86.

Velazquez, E., Igual, J. M., Willems, A., Fernandez, M. P., Munoz, E., Mateos, P. F. Abril, A., Toro, N., Normand, P., Cervantes, E., Gillis, M., \& Martinez-Molina, E. 2001. Description of Mesorhizobium chacoense sp. nov., a novel species that nodulates Propopis alba in the Chaco Arido region (Argentina). Int. J. Syst. Evol. Microbiol. 51: 1011-1021.

Vinuesa, P., Rademaker, J. L. W., de Bruijn, F. J., \& Werner, D. 1998. Genotypic characterization of Bradyrhizobium strains nodulating endemic woody legumes of the Canary Islands by PCR-restriction fragment length polymorphism analysis of genes encoding 16S rRNA (16S rDNA) and 16S-23S rDNA intergenic spacers, repetitive extragenic palindromic PCR genomic fingerprinting and partial 16S rDNA sequencing. Appl. Environ. Microbiol. 64: 20962104.

Vinuesa, P., Silva, C., Werner, D., \& Martinez-Romero, E. 2005a. Population genetics and phylogenetic inference in bacterial molecular systematics: the roles of migration and recombination in Bradyrhizobium species cohesion and delineation. Mol. Phyl. Evol. 34: 29-54.

Vinuesa, P., Leon-Barrios, M., Silva, C., Willems, A., Jarabo-Lorenzo, A., Perez-Galdona, R., Wener, D., \& Martinez-Romero, E. 2005b. Bradyrhizobium canariense sp. nov., an acid-tolerant endosymbiont that nodulates endemic genistoid legumes (Papilionoideae: Genisteae) from the 
Canary Islands, along with Bradyrhizobium japonicum bv. Genistearum, Bradyrhizobium genospecies $\alpha$ and Bradyrhizobium genospecies beta. Int. J. Syst. Evol. Microbiol. 55: 569-575.

Vila, J., Marcos, M. A., \& Jimenez de Anta, M. T. 1996. A comparative study of different PCR-based DNA fingerprinting techniques for typing of Acinetobacter calcoaceticus-A. baumannii complex. J. Med. Microbiol. 44: 482-489.

Vos, P., Hogers, R., Bleeker, M., Reijans, M., van de Lee, T., Hornes, M., Frijters, A., Pot, J., Peleman, J., Muiper, M. \& Zabeau, M. 1995. AFLP: a new concept for DNA fingerprinting. Nucl. Acids Res. 21: 4407-4414.

Wang, E. T., Van Berkum, P., Beyene, D., Sui, X. H., Dorado, O. Chen, W. X., \& MartinezRomero, E. 1998. Rhizobium huautlense sp. Nov., a symbiont of Sesbania herbacea that has a close phylogenetic relationship with Rhizobium galegae. Int. J. Syst. Bacteriol. 48: 687-699.

Wang, E. T., Rogel, M. A., Garcia-de los Santos, A., Martinez-Romero, J., Cevallos, M. A., \& Martinez-Romero, E. 1999a. Rhizobium etli bv. Mimosae, a novel biovar isolated from Mimosa affinis. Int. J. Syst. Bacteriol. 49: 1479-1491.

Wang, E. T., van Berkum, P., Sui, X. H., Beyene, D., Chen, W. X., \& Martinez-Romero, E. 1999b. Diversity of rhizobia associated with Amorpha fruticosa isolated from Chinese soils and description of Mesorhizobium amorphae sp. nov. Int. J. Syst. Bacteriol. 49: 51-65.

Wang, E. T., Martinez-Romero, J., \& Martinez-Romero, E. 1999c. Genetic diversity of rhizobia from Leucaena leucocephala nodules in Mexican soils. Mol. Ecol. 8: 711-724.

Wang, E. T., Tan, Z. Y., Willems, A. Y., Fernandez-Lopez, M., Reinhold-Hurek, B., \& Martinez-Romero, E. 2002. Sinorhizobium morelense sp. nov., a Leucaena leucocephalaassociated bacterium that is highly resistant to multiple antibiotics. Int. J. Syst. Evol. Microbiol. 52: 1687-1693. 
Wang, E. T., Kan, F. L., Tan, Z. Y., Toledo, I., Chen, W. X., \& Martinez-Romero, E.. 2004. Diverse Mesorhizobium plurifarium populations native to Mexican soils. Microbiol. 180: 444454

Wang, F. Q., Wang, E. T., Liu, J., Chen, Q., Sui, H. X., Chen, W. F., \& Chen, W. X. 2007. Mesorhizobium albiziae sp. nov. a novel bacterium that nodulates Albizia kalkora in a subtropical region of China. Int. J. Syst. Evol. Microbiol. 57: 1192-1199.

Wayne, L. G., Brenner, D. J., Colwell, R. R., Grimont, P. A. D., Kandler, O., Krichevsky, M. L. et al. 1987. Report of the ad hoc committee on reconciliation of approaches to bacterial systematics. Int. J. Syst. Bact. 37: 463-464.

Wei, G. H., Wang, E. T., Tan, Z. Y., Zhu, M. E., \& Chen, W. X. 2002. Rhizobium indigoferae sp. nov. and Sinorhizobium kummerowiae sp. nov., respectively isolated from Indigofera spp. and Kummerowia stipulaceae. Int. J. Syst. Evol. Microbiol. 52: 2231-2239.

Wei, G. H., Tan, Z. Y., Zhu, M. E., Wang, E. T., Han, S. Z., \& Chen, W. X. 2003. Characterization of rhizobia isolated from legume species within the genera Astragalus and Lespedeza grown in the Loess Plateau of China and description of Rhizobium loessense sp. nov. Int. J. Syst. Evol. Microbiol. 53: 1575-1583.

Weir, B. S., Turner S. J., Silvester, W. K., Park, D. C., \& Young, J. M. 2004. Unexpectedly diverse Mesorhizobium strains and Rhizobium leguminosarum nodulate native legume genera of New Zealand, while introduced legume weeds are nodulated by Brdyrhizobium species. Appl. Environ. Microbiol. 70: 5980-5987.

Weir, B. S. 2006. Systematics, Specificity, and Ecology of New Zealand Rhizobia. The current taxonomy of rhizobia. http://www.rhizobia.co.nz/taxonomy/rhizobia.html

Welsh, J. \& McClelland, M. 1990. Fingerprinting genomes using PCR with arbitrary primers. Nucl. Acids Res. 18: 7213-7218.

Willems, A., \& Collins, D. 1993. Phylogenetic analysis of rhizobia and agrobacteria based on 16S rRNA gene sequence. Int. J. Syst. Bacteriol. 43: 305-313. 
Willems, A. 2006. The taxonomy of rhizobia: an overview. Plant Soil. 287:3-14.

Willems, A., Doignon-Bourcier, F., Coopman, R., Hoste, B., de Lajudie, P., \& Gillis, M. 2000. AFLP fingerprinting analysis of Bradyrhizobium strains isolated from Faidherbia albida and Aeschynomene species. Syst. Appl. Microbiol. 23: 137-147.

Willems, A., Coopman, R., \& Gillis, M. 2001. Comparison of sequence analysis of 16S-23S rDNA spacer regions, AFLP analysis and DNA-DNA hybridizations in Bradyrhizobium. Int. J. Syst. Evol. Microbiol. 51: 623-632.

Williams, J. G. K., Kubelik, A.R., Livak, K. J., Rafalski, J. A. \& Tingey, S. V. 1990. DNA polymorphisms amplified by arbitrary primers are useful as genetic markers. Nucl. Acids Res. 18: 6531-6535.

Woese, C. R. 1987. Bacterial evolution. Microbiol. Rev. 51: 221-271.

Wojciechowski, M. F. 2003. Reconstructing the phylogeny of legumes (Leguminosae): An early $21^{\text {st }}$ century perspective. In: Klitgaart B. B. \& Bruneau, A. (Eds). Advances in legume systematics, part 10, Higher level systematics. Royal Botanic Gardens, Kew. Pp 5-35.

Wolde-Meskel, E., Terefework, Z., Frostegard, A., \& Lindstrom, K. 2005. Genetic diversity and phylogeny of rhizobia isolated from agroforestry legume species in Southern Ethiopia. Int. J. Syst. Evol. Microbiol. 55 : 1439-1452.

Xu, L. M., Ge, C., Cui, Z., Li, J., \& Fan, H. 1995. Bradyrhizobium liaoningensis sp. nov. isolated from the root nodules soybean. Int. J. Syst. Bacteriol. 45: 706-711.

Yabuuchi, E., Kosako, Y., Yano, I., Hotta, H., \& Nishiuchi, Y. 1995. Transfer of two Burkholderia and an Alcaligenes species to Ralstonia gen. nov.: proposal of Ralstonia picketii (Ralston, Palleroni and Doudoroff 1973) comb. nov., Ralstonia solanacearum (Smith 1896) comb. nov. and Ralstonia eutropha (Davis 1969) comb. nov. Microbiol. Immunol. 39: 897-904. 
Yanagi, M., \& Yamasato K. 1993. Phylogenetic analysis of the family Rhizobiaceae and related bacteria by sequencing of 16S rRNA gene using PCR and DNA sequencer. Microbiol. Lett. 107: 115-120.

Yao, Z. Y., Kan, F. L., Wang, E. T., Wei, G. H., \& Chen, W. X. 2002. Characterization of rhizobia that nodulate legume species of the genus Lespedeza and description of Bradyrhizobium yuanmingense sp. nov. Int. J. Syst. Evol. Microbiol. 52: 2219-2230.

Yates, R. J., Howieson, J. G., Reeve, W. G., Nandasena, K. G., Law, I. J., Brau, L., Ardley, J. K., Nistelberger, H. M., Real, D., \& O'Hara, G. W. 2007. Lotononis angolensis forms nitrogen fixing lupinoid nodules with phylogenetically unique, fast-growing, pink-pigmented bacteria, which do not nodulate L. bainesii or L listii. Soil Biol. Biochem. 39: 1680-1688.

Young, J. P. W. 1996. Phylogeny and taxonomy of rhizobia. Plant Soil. 186: 45-52.

Young, J. P. W., Downer, H. L., \& Eardly, B. D. 1991. Phylogeny of the phototrophic Rhizobium strain BTAi1 by polymerase chain reaction sequencing of a $16 \mathrm{~S}$ rRNA gene segment. Int. J. Syst. Bacteriol. 173: 2271-2277.

Young, J. P .W. \& Haukka, K. E. 1996. Diversity and phylogeny of rhizobia. New Phytol. 133: $87-94$

Young, J. M., Kuykendall, L. D., Martinez-Romero, E., Kerr, A., \& Sawada, H. 2001. A revision of Rhizobium Frank 1889, with an emended description of the genus, and the inclusion of all species of Agrobacterium Conn 1942 and Allorhizobium undicola de Lajudie et al., 1998 as new combinations: Rhizobium radiobacter, $R$. rhizogenes, $R$. rubi, $R$. undicola and $R$. vitis. Int. J. Syst. Evol. Microbiol. 51: 89-103.

Zakhia, F., \& de Lajudie, P. 2001. Taxonomy of rhizobia. Agronomie Rev. 21: 569-576.

Zurdo-pineiro, J. L. Rivas, R., Trujillo, M. E. Viscaino, N., Carrasco, J. A., Chamber, M., Palomares, A., Mateos, P. F., Martinez-Molina, E. \& Velazquez, E. 2007. Ochrobactetrum cytisi sp. nov., isolated from nodules of Cytiasus scoparius in Spain. Int. J. Syst. Evol. Microbiol. 57: 794-798. 


\subsection{TABLES}

Table 1.1. Phenotypic and genotypic methods or characteristics, used for the identification and taxonomic characterization of rhizobia.

\section{Methods or Characteristics Reference}

\section{PHENOTYPIC}

Range of substrates usable as sources of carbon (sugars, sugar alcohols, complex carbohydrates, etc.)

Range of substrates usable as sources of nitrogen (amino acids, urea, nitrate, etc.)

Resistance to specific antibiotics

Electrophoretic mobility of different cell enzymes

Tolerance to different stresses (salt, temperature, $\mathrm{pH}$ )

\section{GENOTYPIC}

Pattern of banding of DNA restriction fragments (genomic-RFLP)

Pattern of banding using REP-PCR primers such as BOX or ERIC

Degree of DNA hybridization

$16 \mathrm{~S}$ rRNA sequence analysis

Sequencing symbiotic and house keeping genes

Genome sequencing
Vandamme et al., 1996; McInroy et al., 1999

Bergersen, 1980

Somasegaren and Hoben , 1994

Bergersen, 1980

Bergersen, 1980

Vandamme et al., 1996

Niemann et al., 1997; Vila et al., 1996 ; Versalovic et al., 1994

Vandamme et al., 1996 ; Wayne et al., 1987

Young et al., 1991; Jarvis et al., 1992; Eardly et al., 1992

Barry et al., 1999 ; Vinuesa et al., 2005; Thies et al., 2001

Vandamme et al.,1996 
Table 1.2. The current taxonomy ${ }^{\mathrm{a}}$, host and references of nitrogen-fixing root nodule bacteria within the $\alpha$ - and $\beta$-subdivision of Proteobacteria.

\begin{tabular}{|c|c|c|c|c|c|}
\hline Division & Family & Genus & Species $^{\mathrm{b}}$ & Legume Host & Reference \\
\hline$\alpha$-Proteobacteria & Rhizobiaceae & Rhizobium & R. leguminosarum bv viciae & $\begin{array}{l}\text { Pisium spp., Vicia spp., } \\
\text { Lathyrus spp., Lens spp. }\end{array}$ & Jordan, 1984 \\
\hline$"$ & $"$ & $"$ & R. leguminosarum bv trifolii & Trifolium spp. & Jordan, 1984 \\
\hline " & $"$ & " & R. leguminosarum bv phaseoli & Phaseolus vulgaris & Jordan, 1984 \\
\hline " & " & " & R. galegae bv officinalis & G. officinalis & $\begin{array}{l}\text { Lindstrom, 1989; } \\
\text { Radeva et al. } 2001\end{array}$ \\
\hline " & $"$ & " & R. galegae bv orientalis & G. orientalis & $\begin{array}{l}\text { Lindstrom, 1989; } \\
\text { Radeva et al., } 2001\end{array}$ \\
\hline$"$ & $"$ & $"$ & R. tropici & $\begin{array}{l}\text { Leucaena spp., Phaseolus } \\
\text { vulgaris }\end{array}$ & $\begin{array}{l}\text { Martinez-Romero et } \\
\text { al., } 1991\end{array}$ \\
\hline " & $"$ & " & R. etli bv phaseoli & Phaseolus vulgaris & Segovia et al., 1993 \\
\hline " & $"$ & " & R. etli bv mimosae & $\begin{array}{l}\text { P. vulgaris,Mimosa affinis } \\
\text { Leucaena } \text { spp. }\end{array}$ & Wang et al., 1999a \\
\hline " & $"$ & " & R. gallicum bv gallicum & $\begin{array}{l}\text { Macroptilium } \\
\text { artroprpureum, } \\
\text { P. vulgaris, Leucaena } \\
\text { leucocephala }\end{array}$ & Amarger et al., 1997 \\
\hline$"$ & $"$ & " & R. gallicum bv phaseoli & $\begin{array}{l}\text { Phaseolus spp., } M . \\
\text { artopurpureum }\end{array}$ & Amarger et al., 1997 \\
\hline " & $"$ & " & R. giardinii bv giardinii & $\begin{array}{l}\text { L. leuococephala, } M \text {. } \\
\text { artopurpureum, }\end{array}$ & Amarger et al., 1997 \\
\hline " & $"$ & " & R. giardinii bv phaseoli & Phaseolus spp. & Amarger et al., 1997 \\
\hline " & " & " & R. hainanense & Desmodium sinuatum & Chen et al., 1997 \\
\hline$"$ & $"$ & " & R. huautlense & $\begin{array}{l}\text { Sesbania herbacea, } L . \\
\text { leucocephala }\end{array}$ & Wang et al, 1998 \\
\hline " & $"$ & " & R. mongolense & $\begin{array}{l}\text { Medicago ruthenica, } \\
\text { Phaseolus vulgaris }\end{array}$ & $\begin{array}{l}\text { Van Berkum et al., } \\
1998\end{array}$ \\
\hline " & $"$ & " & R. yanglingense & $\begin{array}{l}\text { Gueldenstaedtia, Coronilla } \\
\text { varia, Amphicarpaea } \\
\text { triserma }\end{array}$ & Tan et al., 2001 \\
\hline " & $"$ & " & R. indigoferae & $\begin{array}{l}\text { Indigofera spp., } \\
\text { Kummerowia stipulacea }\end{array}$ & Weir et al., 2002 \\
\hline " & " & " & R. cellulosilyticum & Populus alba & $\begin{array}{l}\text { Garcia-Fraile et al., } \\
2007\end{array}$ \\
\hline$"$ & $"$ & " & R. daejeonense & Medicago & Quan et al., 2005 \\
\hline " & $"$ & " & R. loessense & $\begin{array}{l}\text { Astragalus spp., Lespedeza } \\
\text { spp. }\end{array}$ & Wei et al.,2003 \\
\hline " & $"$ & $"$ & R. lusitanum & Phaseolus vulgaris & Valverde et al., 2006 \\
\hline " & " & " & R. sullae & Hedysarum coronarium & Squartine et al.,2002; \\
\hline " & $"$ & $"$ & R. tianshanense & & Chen et al., 1995 \\
\hline " & $"$ & " & R. undicola & Neptunia natans & $\begin{array}{l}\text { Young et al., 2001;De } \\
\text { Lajudie } \text { et al., 1998a }\end{array}$ \\
\hline " & " & Sinorhizobium & S. fredii & $\begin{array}{l}\text { Glycine max, Cajunus } \\
\text { cajam } \\
\text { G. soja, Vigna unguiculata }\end{array}$ & $\begin{array}{l}\text { Scholla and Elka, } \\
1984 \text {; Chen } \text { et al., } \\
1988\end{array}$ \\
\hline " & $"$ & " & S. meliloti & $\begin{array}{l}\text { Medicago spp., Melilotus } \\
\text { spp., Trigonella spp. }\end{array}$ & Jordan, 1984 \\
\hline " & $"$ & " & S. xinjiangensis & $\begin{array}{l}\text { Cajanus cajun, Gycine } \\
\text { max, } \\
\text { G. soja, Vigna unguiculata }\end{array}$ & Chen et al., 1988 \\
\hline " & $"$ & " & S. kostiense & $\begin{array}{l}\text { Acacia spp., Prosopis } \\
\text { chilensis }\end{array}$ & Nick et al., 1999 \\
\hline " & " & " & S. saheli bv acaciae & Acacia spp. & $\begin{array}{l}\text { de Lajudie et al., } \\
\text { 1994; Boivin \& } \\
\text { Giraud, } 1999\end{array}$ \\
\hline$"$ & $"$ & $"$ & S. saheli bv sesbaniae & Sesbabia spp. & $\begin{array}{l}\text { Boivin \& Giraud, } \\
\text { 1999; De Lajudie et } \\
\text { al., 1994 }\end{array}$ \\
\hline$"$ & " & " & S. terangae bv acaciae & Acacia spp. & $\begin{array}{l}\text { De Lajudie et al., } \\
\text { 1994; Lortert } \text { et al., } \\
1996\end{array}$ \\
\hline " & $"$ & " & S. terangae bv sesbaniae & Sesbania spp. & $\begin{array}{l}\text { De Lajudie et al., } \\
1994\end{array}$ \\
\hline " & $"$ & " & S. arboris & $\begin{array}{l}\text { Acacia spp., Prosopis } \\
\text { chilensis }\end{array}$ & Nick et al., 1999 \\
\hline
\end{tabular}


Table 1.2. Continued.

\begin{tabular}{|c|c|c|c|c|c|}
\hline Division & Family & Genus & Species & Legume Host & Reference \\
\hline$"$ & $"$ & $"$ & S. medicae & $\begin{array}{l}\text { Medicago polymorpha, } \\
\text { Medicago spp. }\end{array}$ & Rome et al., 1996 \\
\hline$"$ & $"$ & $"$ & S. morelense & Leucaena leucocephala & Wang et al.,2002 \\
\hline$"$ & $"$ & " & S. kumerowiae & $\begin{array}{l}\text { Kumerowia stipulacea, } \\
\text { Indigofera spp. }\end{array}$ & Wei et al., 2002 \\
\hline$"$ & $"$ & $"$ & S. americanum & $\begin{array}{l}\text { Acacia spp., Phaseolus } \\
\text { vulgaris, Leucaena } \\
\text { leucocephala }\end{array}$ & Toledo et al., 2003 \\
\hline$"$ & $"$ & $"$ & S. arboris & Acacia spp., Prosopis spp. & $\begin{array}{l}\text { Nick et al., } 1999 \\
\text { Young, } 2003\end{array}$ \\
\hline$"$ & $"$ & Mesorhizobium & M. albiziae & Albizia kalkora & Wang et al., 2007 \\
\hline$"$ & $"$ & $"$ & M. loti & $\begin{array}{l}\text { Lotus spp., Lupinus spp., } \\
\text { Anthyllis vulneraria }\end{array}$ & Jarvis et al., 1982 \\
\hline$"$ & $"$ & $"$ & M. huakuii & Astragalus sinicus & Chen et al., 1991 \\
\hline$"$ & $"$ & $"$ & M. ciceri & Cicer arietinum & Nour et al., 1994 \\
\hline$"$ & $"$ & $"$ & M. mediterraneum & Cicer arietinum & Nour et al., 1995 \\
\hline$"$ & $"$ & $"$ & M. tianshaneanse & $\begin{array}{l}\text { Glycine max, Glycyrrhiza, } \\
\text { Caragona polourensins }\end{array}$ & Chen et al., 1995 \\
\hline$"$ & $"$ & $"$ & M. thiogangeticum & Clitoria ternatea & Ghosh \& Roy, 2006 \\
\hline$"$ & $"$ & $"$ & M. amorphae & Amorphae fruticosa & Wang et al., 1999b \\
\hline$"$ & $"$ & $"$ & M. plurifarium & $\begin{array}{l}\text { Acacia spp., Neptunia spp., } \\
\text { Leucaena leucocephala }\end{array}$ & $\begin{array}{l}\text { De Lajudie } \text { et al., } \\
1998 \mathrm{~b}\end{array}$ \\
\hline$"$ & $"$ & $"$ & M. septentrionale & Astragalus adsurgens & Gao et al., 2004 \\
\hline$"$ & $"$ & $"$ & M. temperatum & Astragalus adsurgens & Gao et al., 2004 \\
\hline$"$ & $"$ & Azorhizobium & Az. caulinodans & Sesbania rostrata & Dreyfus et al., 1988 \\
\hline$"$ & $"$ & $"$ & Az. doebereinerae & Sesbania virgata & Moreira et al., 2006 \\
\hline$"$ & Bradyrhizobiaceae & Bradyrhizobium & B. japonicum & $\begin{array}{l}\text { Glycine spp., Macroptilium } \\
\text { artropurpureum }\end{array}$ & Jordan, 1982 \\
\hline$"$ & $"$ & $"$ & B. elkanii & Glycine $\max$ & $\begin{array}{l}\text { Kuykendall et al., } \\
1992\end{array}$ \\
\hline$"$ & $"$ & $"$ & B. betae & Beta vulgaris & Rivas et al.,2004 \\
\hline$"$ & $"$ & $"$ & B. canariense & Lotus spp., Lupinus luteus & Vinuesa et al., 2005 \\
\hline$"$ & $"$ & $"$ & B. lianingense & Glycine $\max , G$. soja & Xu et al., 1995 \\
\hline$"$ & $"$ & $"$ & B. yuanmingense & Lespedeza cuneata & Yao et al., 2002 \\
\hline$"$ & $"$ & $"$ & Bradyrhizobium denitrificans & Aeschynomene indica & $\begin{array}{l}\text { van Berkum and } \\
\text { Eardley, } 2002\end{array}$ \\
\hline$"$ & $\begin{array}{l}\text { Methylobacte- } \\
\quad \text { riaceae }\end{array}$ & Methylobacterium & Methylobacterium nodulans & Crotalaria spp. & Sy et al., 2001 \\
\hline$"$ & $\begin{array}{l}\text { Hyphomicro- } \\
\text { biaceae }\end{array}$ & Devosia & Devosia neptuniae & Neptunia natans & Rivas et al., 2003 \\
\hline$"$ & Brucellaceae & Ochrobactetrum & Ochrobactetrum lupini & $\begin{array}{l}\text { Lupinus albus, Lupinus } \\
\text { spp. }\end{array}$ & Trujillo et al., 2005 \\
\hline$"$ & $"$ & $"$ & Ochrobactetrum cytisi & Cytisus scoparius & $\begin{array}{l}\text { Zurdo-Zurdo-pineiro } \\
\text { et al., } 2007\end{array}$ \\
\hline$"$ & Phyllobacteriaceae & Phyllobacterium & Phyllobacterium trifolii & $\begin{array}{l}\text { Trifolium pretence, } \\
\text { Lupinus albus }\end{array}$ & Valverde et al., 2003 \\
\hline$\beta$-Proteobacteria & Burkholderiaceae & Burkholderia & Burkholderia tuberum & Aspalathus carnosa & $\begin{array}{l}\text { Moulin et al., 2001; } \\
\text { Vandamme et al., } \\
2002\end{array}$ \\
\hline$"$ & $"$ & $"$ & Burkholderia phymatum & Machaerium lunatum & $\begin{array}{l}\text { Vandamme et al., } \\
2002\end{array}$ \\
\hline$"$ & $"$ & $"$ & B. caribensis & $\begin{array}{l}\text { Mimosa diplotricha, } \\
\text { Mimosa pudica }\end{array}$ & $\begin{array}{l}\text { Vandamme } \text { et al., } \\
2002\end{array}$ \\
\hline$"$ & $"$ & $"$ & B.mimosarum & Mimosa pigra, M. scabrello & aChen et al., 2006 \\
\hline$"$ & $"$ & $"$ & B. nodosa & Mimosa bimucronata & Chen et al., 2006 \\
\hline$"$ & " & Cupriavidis & Cupriavidis taiwanensis & $\begin{array}{l}\text { Mimosa pudica, Mimosa } \\
\text { pigra }\end{array}$ & $\begin{array}{l}\text { Chen } \text { et al., } 2001 ; \\
\text { Vandamme \& Coeye, } \\
2004\end{array}$ \\
\hline$"$ & Oxalobac-teraceae & Herbaspirillum & Herbaspirillum lusitanum & Phaseolus vulgaris & Valverde et al., 2003 \\
\hline
\end{tabular}

a Taxonomy as listed in Euzéby's (1997) "List of Prokaryotic names with standing in nomenclature" as updated March, 2008.

b Biovars are indicated as "bv" following the species name. 
Table 1.3. The section, growth habit, and provincial distribution in South Africa of species in the Lebeckia genus (Modified from Germishuizen \& Meyer, 2003).

\begin{tabular}{|c|c|c|c|}
\hline Lebeckia Species & Lebeckia Section & Habit & Province \\
\hline L. acanthoclada & Calobota & Shrubby & Northern Cape \\
\hline L. bowieana & Viborgioides & Shrubby & Northern Cape \\
\hline L. brevicarpa & Lebeckia & Suffrutescent & Western Cape \\
\hline L. carnosa & Lebeckia & Suffrutescent & Northern Cape, Western Cape \\
\hline L. cinerea & Calobota & Shrubby & Northern Cape, Western Cape \\
\hline L. contaminata & Lebeckia & Suffrutescent & Western Cape \\
\hline L. cytisoides & Calobota & Shrubby & Northern Cape, Western Cape \\
\hline L. dinteri & Calobota & Shrubby & Northern Cape \\
\hline L. fasciculata & Viborgioides & Shrubby & Western Cape \\
\hline L. grandiflora & Lebeckia & Suffrutescent & Northern Cape, Western Cape \\
\hline L. halenbergensis & Calobota & Shrubby & Northern Cape \\
\hline L. inflata & Calobota & Shrubby & Western Cape \\
\hline L. leipoldtiana & Viborgioides & Shrubby & Northern Cape, Western Cape \\
\hline L. leptophylla & Viborgioides & Shrubby & Western Cape \\
\hline L. linearifolia & Calobota & Shrubby & Northern Cape, Western Cape \\
\hline L. longipes & Lebeckia & Suffrutescent & Western Cape \\
\hline L. lotonoides & Lebeckia & Suffrutescent & Northern Cape, Western Cape \\
\hline L. macowanii & Lebeckia & Suffrutescent & Western Cape \\
\hline L. macrantha & Stiza & Shrubby & Northern Cape, Eastern Cape \\
\hline L.melilotoides & Calobota & Shrubby & Northern Cape, Western Cape \\
\hline L. meyerianna & Lebeckia & Suffrutescent & Western Cape \\
\hline L. mucronata & Viborgioides & Shrubby & Western Cape, Eastern Cape \\
\hline L. multiflora & Calobota & Shrubby & Northern Cape, Eastern Cape \\
\hline L. obovata & Calobota & Shrubby & Northern Cape \\
\hline L. pauciflora & Lebeckia & Suffrutescent & Western Cape \\
\hline L. plukenetiana & Lebeckia & Suffrutescent & Western Cape \\
\hline L. psiloloba & Stiza & Shrubby & Western Cape, Eastern Cape \\
\hline L. pungens & Stiza & Shrubby & Western Cape \\
\hline L. sepiaria & Lebeckia & Suffrutescent & Western Cape, Eastern Cape \\
\hline L. sericea & Calobota & Shrubby & Northern Cape, Western Cape \\
\hline L. sessilifolia & Viborgioides & Shrubby & Western Cape \\
\hline L. simsiana & Lebeckia & Suffrutescent & Western Cape \\
\hline L.spinescens & Calobota & Shrubby & Northern Cape, Western Cape \\
\hline L. wrightii & Lebeckia & Suffrutescent & Western Cape \\
\hline
\end{tabular}




\subsection{FIGURES}

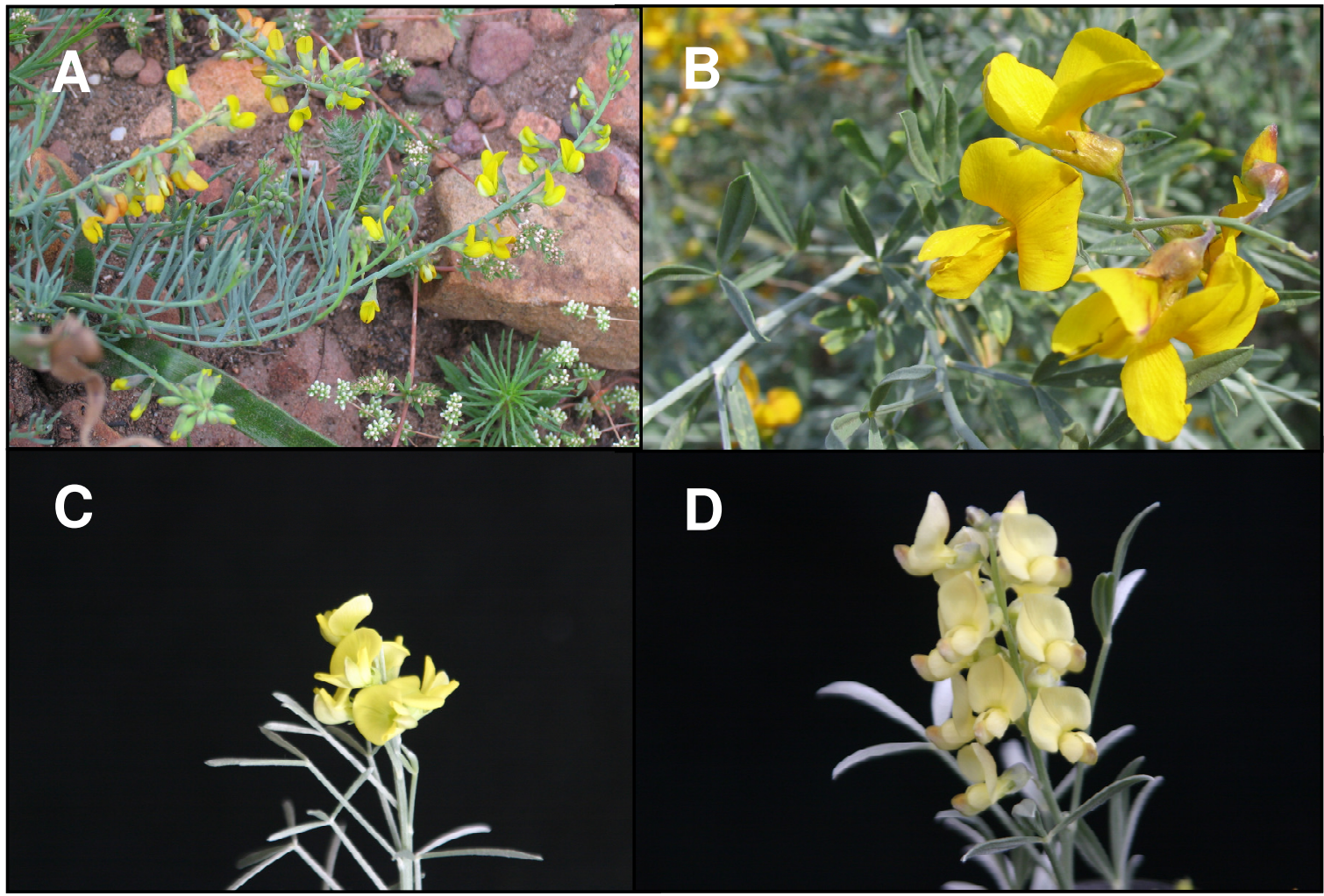

Figure 1.1. Examples of the spikes of yellow flowers characteristic of Lebeckia species found in the Western Cape. A: L. plukenetiana, a suffrutescent shrub with needle leaves (Lebeckiasuffrutescent). B: L. cytisoides, a shrub with trifoliate leaves (Calabota-shrubby); C: L. multiflora, shrub with pea-shaped flowers (Calabota-shrubby) and trifoliate leaves. D: $L$. sericea, shrub with trifoliate leaves (Calabota-shrubby), Photos courtesy of S. Boatwright (A, B) and K. Kasdorf (C, D). 


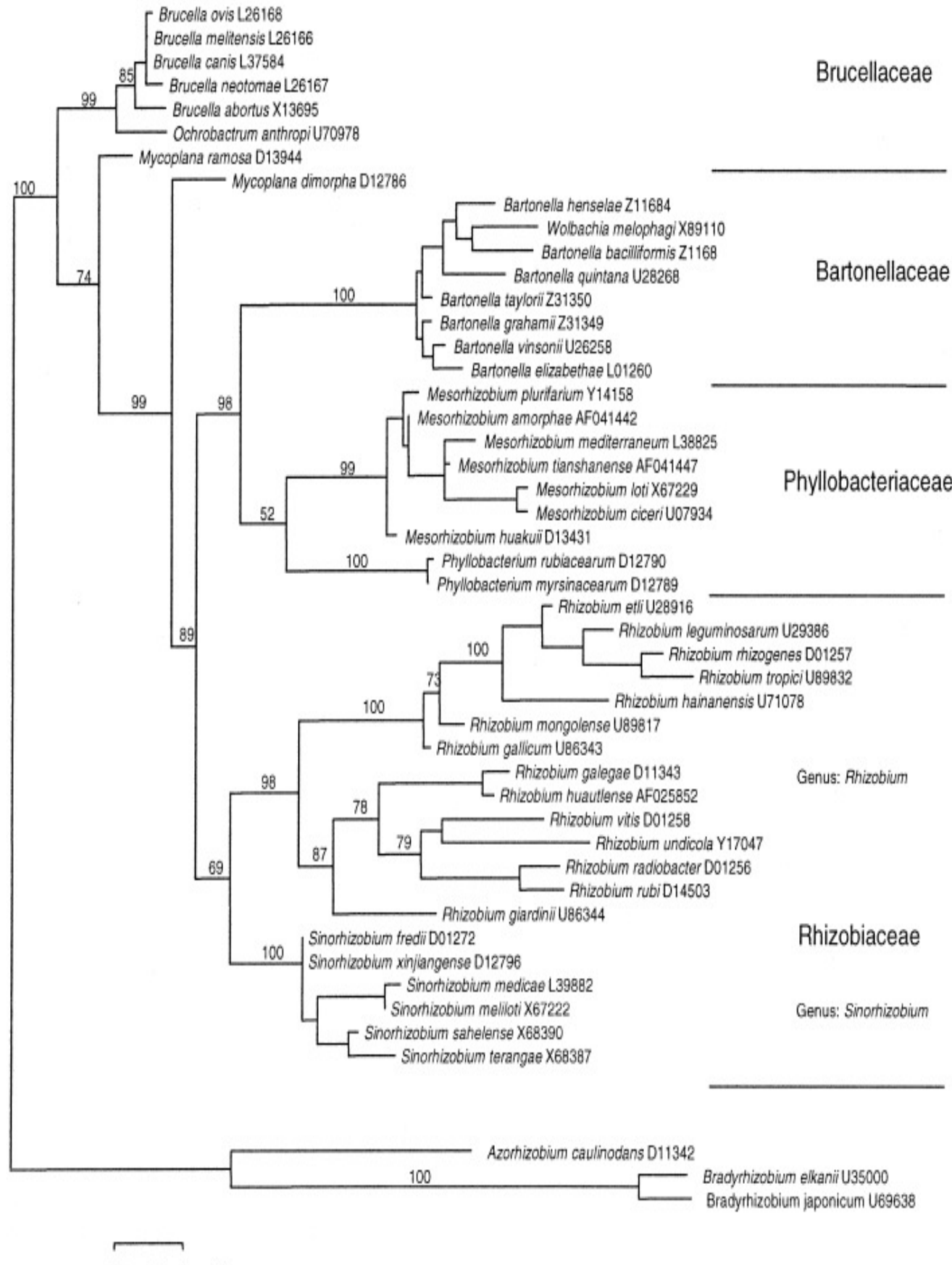

0.01 substitutions / site

Figure 1.2. Neighbour-joining tree of Rhizobiaceae and relatives based on 16S rRNA sequences (Young et al., 2001). 


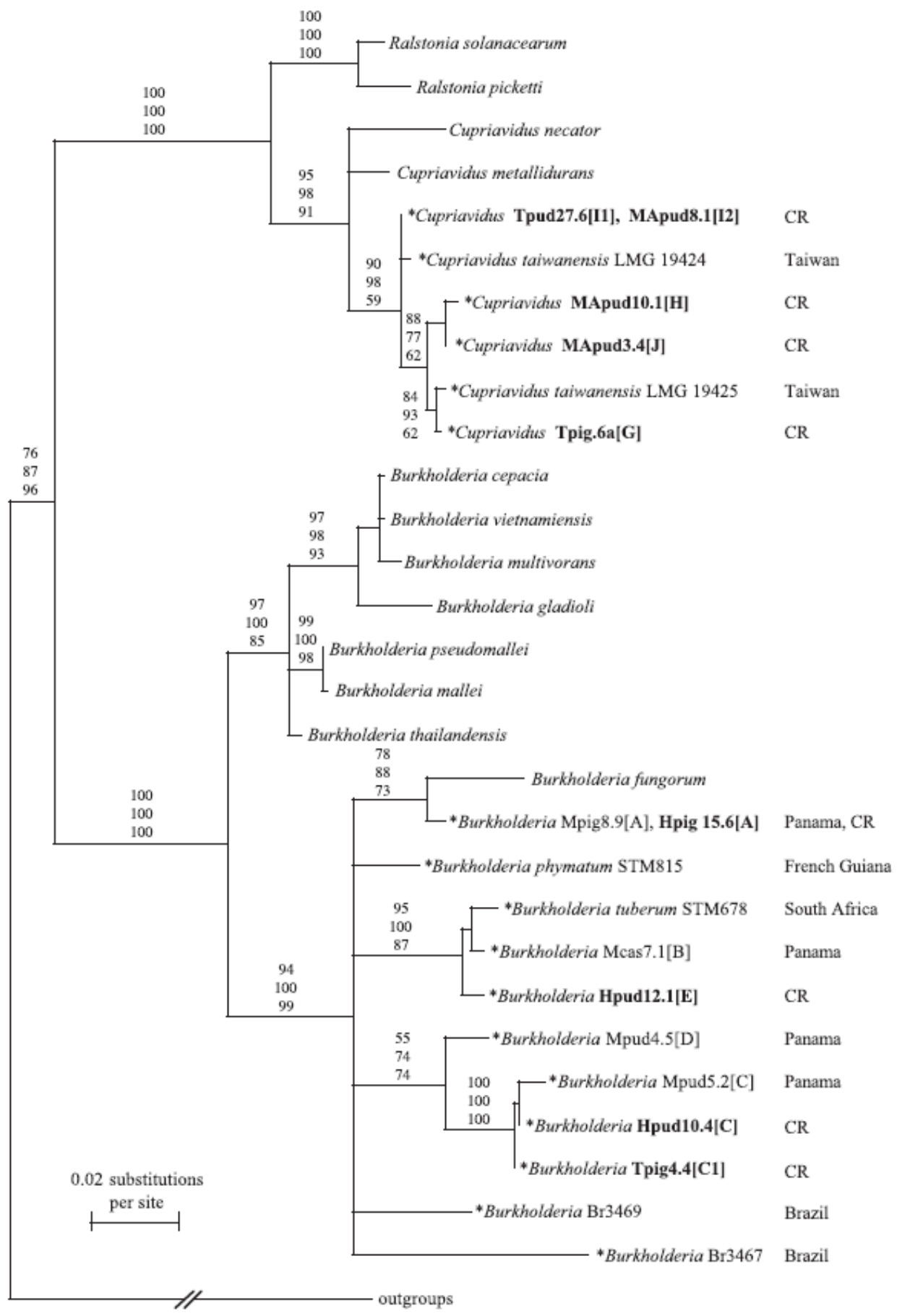

Figure 1.3. Maximum likelihood analysis of 16S rRNA genes of Burkholderia, Cupriavidis and other $\beta$-Proteobacteria (Barrett \& Parker, 2006). 


\begin{tabular}{|c|c|c|c|c|}
\hline & Family Genus & Species & Subspecies & Strain \\
\hline \multicolumn{5}{|l|}{ DNA-DNA Hybridization } \\
\hline \multicolumn{5}{|l|}{ ARDRA } \\
\hline \multicolumn{5}{|l|}{ House-keeping gene sequences } \\
\hline \multicolumn{5}{|l|}{ 16S rRNA sequences } \\
\hline \multicolumn{5}{|l|}{ ITS-PCR } \\
\hline \multicolumn{5}{|l|}{ RFLP } \\
\hline \multicolumn{5}{|l|}{ Whole-cell protein profile } \\
\hline \multicolumn{5}{|l|}{ AFLP } \\
\hline \multicolumn{5}{|l|}{ RAPD } \\
\hline REP-PCR & & & & \\
\hline
\end{tabular}

Figure 1.4. The relative taxonomic resolving power of various fingerprinting and sequencing techniques (modified from Rademaker \& de Bruijn, 1997). 
CHAPTER 2

ISOLATION OF RHIZOBIA FROM LEBECKIA SPECIES INDIGENOUS TO SOUTH AFRICA AND THEIR NODULATION PROPERTIES ON LEBECKIA AND THE PROMISCUOUS LEGUMES COWPEA AND SIRATRO

\section{TABLE OF CONTENTS}

2.1 ABSTRACT

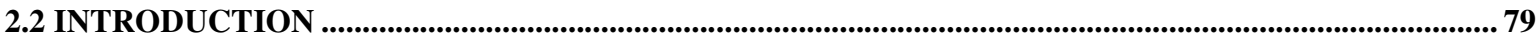

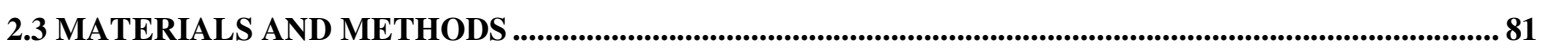

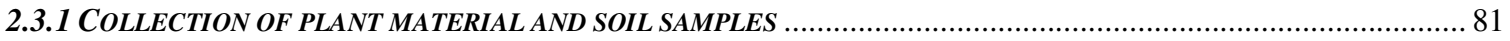

2.3.2 SEED GERMINATION, RHIZOBIAL TRAPPING AND PLANT GROWTH CONDITIONS ...................................... 81

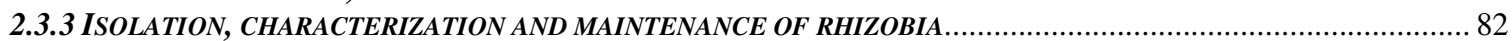

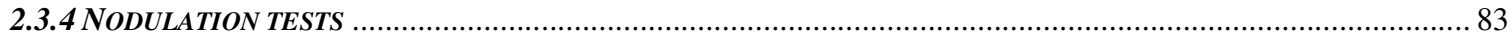

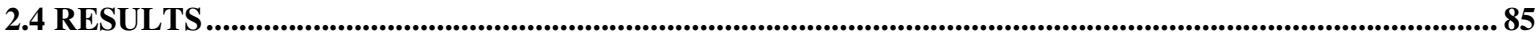

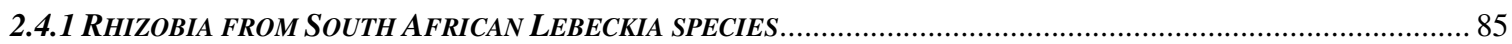

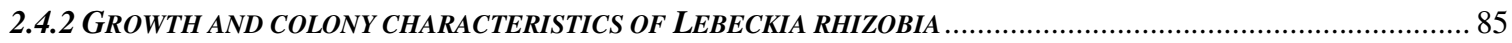

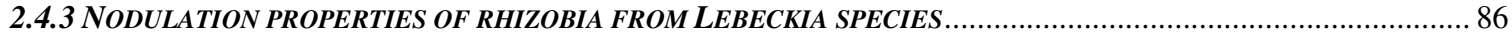

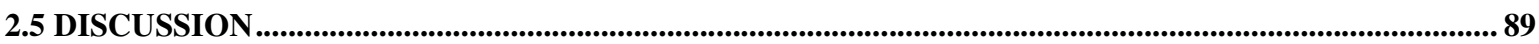

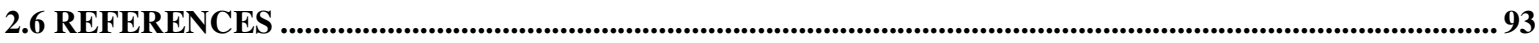

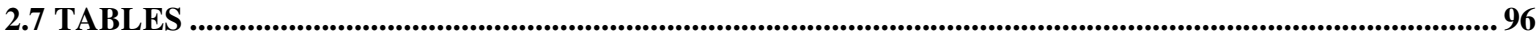

2.8 FIGURES 


\subsection{ABSTRACT}

The genus Lebeckia (family Leguminosae, tribe Crotalarieae) contains close to 35 shrubby and herbaceous legume species native to the Western Cape region of South Africa. Members of this genus typically have spikes of yellow pea-flowers and are divided into five sections based on growth habit and leaf shape. The shrubby trifoliate-leaf species are included in the sections Viborgioides, Calobota and Stiza and herbaceous needle-leaf species in the sections Lebeckia and Spira. Many Lebeckia species are recognized as valuable forage plants and, like most other legumes, Lebeckia species also form symbiotic relationships with rhizobial bacteria that fix atmospheric nitrogen in root structures called nodules. Any effort to exploit these plants for agricultural purposes would therefore require knowledge of the specificity of Lebeckia for their rhizobial symbionts. As a first step towards this goal, the nodulation specificity of the rhizobia associated with ten Lebeckia species representing three sections (Calobota, Stiza and Lebeckia) and the two growth habits were studied. A total of 79 Gram-negative rhizobia were isolated from these plants, after which their nodulation abilities were confirmed on their homologous hosts and the promiscuous legumes cowpea and siratro. These tests suggested some degree of specificity, as $56 \%$ of the strains were effective on cowpea and $77 \%$ on siratro, while all effectively nodulated their homologous Lebeckia hosts. This nodulation specificity was even more evident from the results of cross-inoculation studies where rhizobia isolated from shrubby Lebeckia species were not able to effectively nodulate suffrutescent Lebeckia species and vice versa. Root nodules were formed when rhizobia from Lebeckia species with a specific growth habit were inoculated onto other species with the same habit, but nodulation was most effective when rhizobia from a specific section were inoculated onto other members of that section. This is the first report on nitrogen fixation and nodulation by rhizobia of the genus Lebeckia and the results suggest that the apparent specificity between the two symbiotic partners will complicate commercial inoculation of these legumes.

Keywords: Crotalarieae, Lebeckia, cowpea, siratro, nodulation, nitrogen fixation 


\subsection{INTRODUCTION}

Lebeckia species are evergreen shrubby or herbaceous (suffrutescent) legumes of the family Fabaceae and tribe Crotalarieae, which are mainly indigenous to the southern and Western Cape regions of South Africa (Germishuizen \& Meyer 2003). The genus consists of about 33-36 species (Germishuizen \& Meyer, 2003; Boatwright \& van Wyk, 2007; Boatwright et al., 2007; Le Roux \& van Wyk, 2007; Le Roux et al., 2007) and is presently undergoing taxonomic revision by researchers in the Botany Department, University of Johannesburg (van Wyk, personal communication; Le Roux \& van Wyk, 2007; Boatwright et al., 2007). Plants of this genus generally flower in spring and early summer and are characterized by spikes of yellow pea-flowers (Fig. 2.1). Based on morphological differences Lebeckia species are divided into the five sections Calobota, Stiza, Lebeckia, Spira and Viborgioides (Bentham, 1844; Harvey, 1862; Le Roux \& van Wyk, 2007; Boatwright et al., 2007; van Wyk, personal communication). Of these, shrubby trifoliate-leaf species are included in the sections Calobota and Stiza and Viborgioides (Bentham, 1844; Harvey, 1862) and suffrutescent needle-leaf species in the sections Lebeckia and Spira (Le Roux \& van Wyk, 2007; van Wyk, personal communication).

Various Lebeckia species are recognized as valuable forage crops (Le Roux et al., 1994; Shearing \& van Heerden, 1994). In the veld, species such as L. spinescens and L. multiflora provide nutritious grazing, although other species such as L. cytisoides are toxic (Van Breda \& Barnard, 1991; Le Roux et al., 1994; Shearing \& van Heerden, 1994). Due to the beautiful flowers of some species (e.g. L. sericea), they are also popular garden plants and suitable for commercial sale in garden nurseries. From an ecological point of view, all Lebeckia species are likely to also be of great value because of their nitrogen-fixing symbiosis with bacteria known as rhizobia (Corby et al., 1983; Le Roux et al., 1994) and consequent contribution to soil fertility and soil health maintenance (Le Roux et al., 1994; Shearing \& van Heerden, 1994). Any effort to exploit Lebeckia for agricultural and other purposes would however require knowledge of the level of specificity between the plant and rhizobial partners. Development of rhizobial inoculants will also involve selection of bacterial strains that are highly effective in their association with their compatible host.

As a first step to potentially developing inoculant strains for Lebeckia, the aim of this study was to determine the level of specificity between Lebeckia species and their symbiotic diazotrophic rhizobial partners. For this purpose, ten indigenous wild species of Lebeckia were selected from 
the three sections Calobota, Stiza and Lebeckia for which seed or soil from collection sites for trapping of rhizobia were available. Five shrubby species (L. cytisoides, L. multiflora, L. sericea, L. spinescens and L. pungens) and five suffrutescent species (L. ambigua, L. sepiaria, L. pauciflora, L. meyerianna and L. simsiana) were used. Once rhizobia were obtained from the root nodules of these plants and their nodulation abilities were confirmed in nitrogen-free environments, their specificity towards other Lebeckia species and the promiscuously nodulating legumes siratro (Macroptilium atropurpureum, tribe Phaseoleae) and cowpea (Vigna unguilata, tribe Phaseoleae) were investigated. 


\subsection{MATERIALS AND METHODS}

\subsubsection{Collection of plant material and soil samples}

Plant specimens, including flowers, leaves, seeds and root nodules were collected during the spring and summer seasons of 2004 to 2006 in diverse localities ranging from rocky Karoo plains and hills, sandy veld inland of Lambert's Bay and rugged mountain terrain of the Cedarberg and Namaqualand in South Africa. A map of the nine collection sites is shown in Fig. 2.2 and some locality details are provided in Table 2.1. The majority of the plants ( $L$. meyerianna, L. pauciflora, L. cytisoides, L. sepiaria, and L. multiflora) were collected in the Western Cape at Clanwilliam, Cedarberg, Citrusdal and Lambert's Bay, while Lebeckia sericea was collected at Bitterfontein in the North Cape. The L. ambigua and L. simsiana specimens came from Modder River and Stellenbosch in the south of the Western Cape and L. spinescens and L. pungens in the east of the Western Cape at Beaufort West and Meiringspoort, respectively. Plant species were identified by Professor B.-E. van Wyk and co-workers in the Department of Botany, University of Johannesburg. At each site, soil was collected next to the roots of the plants at depths of 5-30 cm and later used in conjunction with the appropriate host species to trap rhizobia (see below). Nodules were carefully excised from roots and stored in vials containing silica gel desiccant. In some instances, small seedlings were potted in soil and transported to the greenhouse for growth and later examination for nodules. Soil $\mathrm{pH}$ was measured using 1:5 soil: $\mathrm{H}_{2} \mathrm{O}$ mixtures, after shaking for $30 \mathrm{~min}$ and leaving to stand for $3 \mathrm{~h}$ at room temperature. Siratro and cowpea seed were obtained from the Agricultural Research Council-Range and Forage Institute (ARC-RFI) Seed Genebank, Pretoria, South Africa.

\subsubsection{Seed germination, rhizobial trapping and plant growth conditions}

Lebeckia seeds used for trapping experiments and nodulation tests were either collected in the field or kindly donated by Professor B.-E. van Wyk. These seeds were pretreated with concentrated sulphuric acid $\left(\mathrm{H}_{2} \mathrm{SO}_{4}\right)$ for 20-30 min, washed 5 times with sterile deionised water (SDW) and imbibed in SDW for 3-4 h. Siratro and cowpea seeds were also prepared for germination. The siratro seed was treated with $\mathrm{H}_{2} \mathrm{SO}_{4}$ for 15 min and washed with SDW, after which the seed was surface sterilized in $3.5 \%(\mathrm{~m} / \mathrm{v})$ sodium hypochlorite for 5-10 min, followed by washing and imbibition as described for Lebeckia seeds. Cowpea seed was surface sterilized with $3.5 \%(\mathrm{~m} / \mathrm{v})$ sodium hypochloride for $10 \mathrm{~min}$ and washed and imbibed as before. After imbibition, seeds of Lebeckia were germinated in the dark under aseptic conditions for 3-5 d at 
$15^{\circ} \mathrm{C}$ on the surface of water agar (WA; $1.5 \%, \mathrm{~m} / \mathrm{v}$, Associated Chemical Enterprises, RSA). Cowpea and siratro seeds were germinated at $28^{\circ} \mathrm{C}$ on WA for 1 and $5 \mathrm{~d}$, respectively.

For the trapping experiments and for testing nodulation and nitrogen-fixing effectiveness of the isolated bacteria, two seedlings of a specific Lebeckia species, or cowpea or siratro, were planted in Leonard jars containing quartz sand and nitrogen-free Hoagland solution (Somasegaren \& Hoben, 1994). For the trapping experiments, the jars were then inoculated with a suspension of soil from a locality corresponding to that of the seed. The suspension was prepared by shaking $10 \mathrm{~g}$ soil in $100 \mathrm{ml}$ water for $30 \mathrm{~min}$. After sedimentation, the liquid supernatant was pipetted onto the seedlings. Alternatively, sand in the Leonard jar was scooped out and replaced with a plug of soil from the locality where the Lebeckia species was collected. For testing the nodulation and nitrogen-fixing effectiveness of the isolated bacteria (see below), seedlings were inoculated with inoculum prepared as described below and covered with a Petri dish. Once seedlings had reached appreciable height, the glass Petri dish cover of the Leonard jar was removed and sterile sand coated with paraffin wax sand added to cover the sandy part of the jar. Its purpose was to seal the sand in the Leonard jars after plants have emerged to reduce loss of moisture and prevent microbial contamination. The glasshouse was set at a $14 \mathrm{~h}$ day temperature of $28^{\circ} \mathrm{C}$ and a $10 \mathrm{~h}$ night temperature of $15^{\circ} \mathrm{C}$. Plants were grown for $4-12$ weeks before nodule harvest.

\subsubsection{Isolation, characterization and maintenance of rhizobia}

Nodulated roots were washed free of sand or soil and nodules carefully excised. For isolation of rhizobia, whole nodules were surface sterilized for $2-3 \mathrm{~min}$ in $3.5 \%(\mathrm{~m} / \mathrm{v})$ sodium hypochlorite. After washing in 5 changes of SDW, isolations were performed by squashing individual nodules in a drop of SDW with sterile forceps and streaking the extracts onto Yeast Mannitol Congo red (YM-CR) agar plates (Somasagaren \& Hoben, 1994). Cultures were incubated at $28^{\circ} \mathrm{C}$ until there was sufficient bacterial growth to form colonies. The cultures were purified on YM-CR by single colony isolation and stored at $-70^{\circ} \mathrm{C}$ using sterile $20 \%(\mathrm{v} / \mathrm{v})$ glycerol as a cryoprotectant. All isolates (Table 2.2) are stored in a $-70^{\circ} \mathrm{C}$ freezer at the South African Rhizobium Collection (SARC), Plant Protection Research Institute, Pretoria, South Africa. Two isolates previously isolated from Lebeckia species were obtained from the SARC and included in this study. The strain XHR1 (L. simsiana) was collected by C. Deschodt in 1979 and strain NK22 (L. sericea) was collected by J. J. le Roux in 2003. 
All isolates were characterized using standard Gram stains (Preston \& Morrell, 1962). This briefly entailed preparing a smear of a moderately dense suspension of bacteria in SDW on a clean slide and air drying it. After fixation with heat, $0.5 \%(\mathrm{~m} / \mathrm{v})$ crystal violet was applied to the smear for $1 \mathrm{~min}$, after which it was rinsed with tap water. A few drops of iodine $(0.5 \%, \mathrm{v} / \mathrm{v})$ were then applied to the smear for $1 \mathrm{~min}$, followed again by rinsing with tap water. To remove any of the non-absorbed dye, 95\% ethanol was applied to the smear for $30 \mathrm{sec}$, after which several drops of $0.5 \%(\mathrm{~m} / \mathrm{v})$ Safranin O was applied to the smear for $10 \mathrm{sec}$. Following rinsing and drying, the slide was mounted in oil and observed using a Zeiss compound microscope at 1000X magnification. Cells which absorbed the crystal violet dye were purple and considered Gram positive, whereas those that were red were Gram negative. The alternative 3\% $\mathrm{KOH}$ test to confirm whether bacterial cells are Gram negative or positive was also used (Halebian et al., 1981; Goszczynska et al., 2000). A drop of $3 \% \mathrm{KOH}$ was placed on a slide and mixed with a loopful of bacterial culture. The loop was lifted several times to check for formation of a mucoid thread. Bacterial strains that produce a mucoid thread in $3 \% \mathrm{KOH}$ are Gram negative, whereas those that form a watery suspension are Gram positive (Bamarouf et al., 1996; Goszczynska et al., 2000).

The morphology of colonies of individual rhizobial isolates on YM-CR medium was observed using a microscope with 100X magnification (Wild Heerbrugg, Switzerland). Relative colony growth rates were evaluated by measuring the diameter of the colonies at three days and six days after streaking. The isolates were regarded as fast-growing if they produced discernable colonies within 3 days and slow-growing when colonies were observed after 5 days.

\subsubsection{Nodulation tests}

To authenticate the nodulation and nitrogen-fixing abilities of the isolates used in this study (Table 2.1), all isolates were inoculated onto their homologous or compatible hosts. For this purpose inoculum was prepared by suspending two loopfulls of a fresh single colony culture from an YM-CR agar plate in $4 \mathrm{ml}$ sterile distilled water (SDW) in a McCartney bottle. The suspensions were mixed by vortexing and $1 \mathrm{ml}$ inoculated onto each seedling planted in a Leonard jar, after which seedlings were grown as described above. The isolated rhizobia were also inoculated onto the promiscuous legumes cowpea and siratro to test for host range specificity of nodulation and nitrogen fixation effectiveness. All nodulation experiments 
contained at least four replicate Leonard jars per treatment, with two to four seedlings per jar. The plants from the four replicate Leonard jars were compared to verify the results.

To determine the symbiotic specificity between the various rhizobial isolates and their plant hosts, cross-inoculation experiments were performed using only those Lebeckia species for which sufficient seed was available. Three experimental sets of hosts and rhizobia were used. In the first set (Set 1), inoculum prepared with rhizobia isolated from shrubby hosts in either of the sections Calobota (L. cytisoides, L. multiflora, L. sericea and L. spinescens) and Stiza (L. pungens) were used to inoculate other species from either the same section or from the other sections. In the second experiment (Set 2), inoculum prepared with rhizobia isolated from the suffrutescent hosts in section Lebeckia was used to inoculate other species from this section $(L$. meyerianna, L. ambigua and L. simsiana). In the third experiment (Set 3), inoculum prepared with rhizobia isolated from both the suffrutescent and the shrubby Lebeckia sections were used to inoculate shrubby and suffrutescent Lebeckia species, respectively. These inoculations were also performed in the opposite direction, with inoculum prepared with rhizobia isolated from the shrubby and suffrutescent Lebeckia sections being used to inoculate suffrutescent and shrubby Lebeckia species, respectively.

All plants were grown under nitrogen-free conditions as described above, during which nitrogenfixation was monitored in inoculated plants by comparing the greenness of their leaf colour with the yellow leaves of uninoculated control plants. In addition, harvested nodules were sliced in half with a scalpel and examined. A pink coloration, caused by the production of leghaemoglobin was indicative of an effective nitrogen-fixing symbiosis while a white colour signified an ineffective association (Somasagaren \& Hoben, 1994; Sprent, 2007; Sprent \& James, 2007). In all cases rhizobia were re-isolated from the root nodules induced and plated onto YM-CR, after which the colonies that emerged were compared for microscopic appearance and rate of growth to that of the original cultures used to inoculate test plants. 


\subsection{RESULTS}

\subsubsection{Rhizobia from South African Lebeckia species}

In this study, 79 strains of rhizobia (Table 2.2) were isolated from the root nodules of ten Lebeckia species collected from different localities in South Africa (Table 2.1 and Fig. 2.2). The soil types at these collection sites ranged from sandy to sandy clay loam to stony clay loam that were mostly moderately acidic with pH 5.25-6.5 (Table 2.1). Two strains were extracted from $L$. cytisoides collected from Citrusdal, as well as one isolate from L. sepiaria also from the same locality (Table 2.2). L. multiflora was collected at Nortier Research Station, Lambert's Bay and four rhizobial strains were purified from root nodules. L. sericea was the most abundant of the Lebeckia species studied and was found in two localities, Bitterfontein and Kamiesberg. A total of eighteen isolates were extracted from L. sericea. L. spinescens was abundant at Bleak House farm, Beaufort West and sixteen rhizobial strains were obtained from this plant. Ten strains came from L. pungens collected at Meiringspoort. L. ambigua was collected from Modder River and nine rhizobial strains were obtained. L. pauciflora was collected in the Cedarberg and twelve strains were studied. L. meyerianna was collected at Clanwilliam with four isolates being purified from nodules. SARC records show that the two L. simsiana strains were collected from a Stellenbosch garden shrub.

\subsubsection{Growth and colony characteristics of Lebeckia rhizobia}

All the strains isolated from the Lebeckia root nodules were Gram negative rods, as they all appeared red upon staining with Safranin O. They also reacted positively to the $3 \% \mathrm{KOH}$ test, with mucoid threads being observed in all. The colonies also did not absorb Congo red on YM, were pale yellow to white in colour and were generally low convex in shape with entire edges. All the rhizobia reisolated from the cowpea, siratro and Lebeckia nodules had colony characteristics similar to those of the original isolates from host plants when cultured on YMCR.

In terms of growth rate on YM-CR agar, the bacteria isolated in this study varied substantially (Tables 2.2, 2.3). Of the Lebeckia spp. studied, three from section Calobota yielded intermediate growers and one fast grower, while the single species studied in section Stiza was nodulated by very slow growers. In addition, two of the five species from section Lebeckia yielded fast growers, two yielded slow growers, and L. pauciflora was nodulated by both fast and slowgrowers (Tables 2.2, 2.3). Nineteen percent of all the strains studied were slow growers, while 
$30 \%$ were intermediate growers reaching colony diameters of $1-2 \mathrm{~mm}$ within 5 to $6 \mathrm{~d}$. The remaining $51 \%$ were fast-growers. Fifteen slow-growing strains from $L$. pungens (10 strains; Betal106a to Betal106j), L. sepiaria (WC12.1a), L. pauciflora (WC21.1k and WC21.11) and $L$. simsiana (XHR1a and XHR1b) had colony diameters less than or equal to $1 \mathrm{~mm}$ at 6 to $8 \mathrm{~d}$. In addition to the two slow-growing $L$. pauciflora strains mentioned above, isolates from $L$. pauciflora included 10 fast-growing strains (WC21.1a-j). The twenty four intermediate-growing strains included those isolated from L. cytisoides (2 strains; WC19.1b and WC19.1c), L. multiflora (4 strains; WC 23.1a to WC23.1d) and L. sericea (18 strains; WC28.1a to WC28.1j, WC33a to WC33h, and NK22). Approximately half of the Lebeckia rhizobia were fast growers (Table 2.2) and were isolated from four Lebeckia species, i.e. L. spinescens (16 strains; BH1LS to BW3S), L. ambigua (9 strains; WC5.4a to WC5.4i), L. pauciflora (10 strains; WC21.1a to WC21.1j) and L. meyerianna (4 strains; WC26.1c to WC26.1f). The fast-growing isolates from L. spinescens were watery mucoid with both large and small colonies and were $3 \mathrm{~mm}$ in diameter at 2-3 days (Table 2.2). The remaining 23 fast-growing strains from L. ambigua, L. pauciflora and L. meyerianna had an almost identical growth rate, with colonies developed at four days.

\subsubsection{Nodulation properties of rhizobia from Lebeckia species}

Representative bacterial strains originating from the root nodules of each species of Lebeckia were used to inoculate their corresponding hosts as well as cowpea and siratro. All were found to be effective on their homologous host plant, producing nitrogen-fixing nodules within a minimum of 6 weeks (Figs. 2.3 and 2.4, Table 2.3). When the root nodule isolates were tested for nitrogen fixation and nodulation on cowpea and siratro, many of the isolates were effective (i.e. stimulated production of green leaves and pink centered nodules indicating nitrogen fixation), whereas others had stunted growth and yellow leaves indicating ineffective nodulation or failure to nodulate. Overall nodulation of cowpea and siratro by the rhizobia from Lebeckia was similar, with little variation in nodulation compatibilities by certain strains. Siratro was, however, more promiscuous than cowpea when inoculated with Lebeckia rhizobia, as a higher percentage of the strains were effective on siratro (77\%) than on cowpea (56\%). No obvious correlation was observed between growth rate of the isolates and their ability to nodulate cowpea and siratro except that \% effectiveness was sometimes lower amongst fast growers, such as the L. spinescens isolates (Table 2.3). 
Some isolates appeared not to be able to nodulate the promiscuous hosts siratro and cowpea. For example, intermediate-growing isolates from L. cytisoides nodulated neither cowpea nor siratro, while the single isolate from L. sepiaria did not nodulate siratro but produced effective nodules on cowpea (Table 2.3). All four intermediate-growing strains from L. multiflora formed an effective symbiosis with siratro, while on cowpea only two were effective with the other two producing ineffective nodules. Eighteen intermediate-growing strains from L. sericea, nodulated cowpea and siratro although only $50 \%$ fixed nitrogen on cowpea and $66 \%$ on siratro (Table 2.3). Similarly, all 16 fast-growing strains from $L$. spinescens nodulated cowpea and siratro, but only $12 \%$ were effective on cowpea, whereas $75 \%$ were effective on siratro. In contrast, the fastgrowing isolates from L. pauciflora and L. meyerianna were similar in effectiveness on cowpea and siratro plants, both obtaining $60-80 \%$ effective nodulation on these species. (Table 2.3). The two slow-growing strains from L. pauciflora produced effective nodules on both cowpea and siratro. The 10 slow-growing isolates from L. pungens were $80 \%$ effective on cowpea and $100 \%$ effective on siratro, while the two slow-growing L. simsiana isolates, as well as the two slow-growing L pauciflora isolates, were effective on both cowpea and siratro. All nine slowgrowing $L$. ambigua rhizobia tested fixed nitrogen on siratro whereas only six of the nine strains were effective on cowpea (Table 2.3).

Inoculation of the rhizobia on to their homologous Lebeckia hosts resulted in the formation of indeterminate nodules (Fig. 2.4; Corby et al., 1983). Some of these were elongate and flat finger-like or leg-like, while a few were branched, ovoid-cylindrical and laterally flat. The majority of the Lebeckia species, however, produced large fan-like nodules with separate lobeshaped branches. The fan-shaped nodules were densely aggregated with 4-6 bulbous fingers and a single narrow connection to the root (Fig. 2.4). Inoculation of the Lebeckia rhizobia onto the promiscuous phaseoloid legumes produced spherical and clearly determinate nodules on cowpea (Fig 2.4; Corby et al., 1983) and ovate to cylindrical indeterminate nodules on siratro (Fig. 2.4).

Representative isolates and plants from the two Lebeckia growth habits (shrubby trifoliate leaf and suffrutescent needle leaf) were used to perform cross-inoculation tests. In control experiments, the isolates were reinoculated on their respective homologous host plants to confirm their nodulation and nitrogen fixation abilities on their corresponding host (Table 2.3; Figs. 2.3, 2.4). Three sets of experiments were conducted, using only those plant species for which adequate seed was available. The first two sets of cross-inoculation tests (Sets 1 and 2; 
Table 2.4) were performed among the plants and their respective rhizobia within each of the shrubby (sections Stiza and Calobota) or suffrutescent (section Lebeckia) growth habits. These cross-inoculations all resulted in the formation of root nodules. Inoculations performed with isolates from shrubby section Calobota plants onto species from section Calobota induced the production of effective nodules. The same was also true for inoculations performed with isolates from species of the suffrutescent section Lebeckia and host plants representing this section (Table 2.4). However, inoculations performed with isolates from shrubby section Calobota plants onto species of the shrubby section Stiza, and vice versa, induced production of ineffective nodules (Table 2.4). In a third set, suffrutescent section Lebeckia was inoculated with rhizobia from the shrubby section Calobota, and a reverse set of legumes from shrubby sections Stiza and Calobota inoculated with isolates from species in suffrutescent section Lebeckia (Set 3; Table 2.4). For this experimental set, no nodules were observed on the roots of the test plants (Table 2.4). The only exception was inoculation of L. pungens (Section Stiza; shrubby habit) with WC26.1e originating from L. meyerianna (Section Lebeckia; suffrutescent habit), which generated ineffective nodules (Table 2.4). 


\subsection{DISCUSSION}

In this study, 79 isolates of rhizobial bacteria were obtained from the root nodules of ten species of Lebeckia. However, before these bacteria could be regarded as rhizobia, their nodulation properties had to be authenticated. Verification of the nodulation abilities of bacteria associated with root nodules of legumes represents an essential first step in characterizing the nitrogenfixing symbiosis. The main reason for this is that the soil environment harbours diverse microorganisms and non-rhizobial contaminants are easily obtained during the isolation process. To ensure that all the isolates used in this study were authentic symbiotic root nodule bacteria of Lebeckia, the isolates were inoculated on their corresponding host plants. Each was found to produce effective nodules that stimulated shoot growth of the host. Examination of the cultural characteristics of the rhizobia re-isolated from these nodules also confirmed that they corresponded to those of the parental isolates (Tables 2.2, 2.3). These observations fulfilled Koch's postulates and indicated that the isolates were authentic root nodule bacteria of the Lebeckia species studied.

In this study, all the rhizobia isolated from the Lebeckia species examined were Gram-negative, a feature they share with all legume root nodule bacteria examined to date. These bacteria did, however, differ considerably from one another in terms of culture characteristics, growth rate and colony appearance (Table 2.3). Based on growth rate, the isolates examined were separated into a number of groups. These groupings corresponded to the slow- and medium-growing groupings that are, respectively, characteristic of bradyrhizobia and mesorhizobia, as well as the fast-growers found in some genera of root nodule bacteria especially Rhizobium and Burkholderia (Barrett \& Parker, 2006). Where more than one isolate was obtained from a plant species, all had similar growth characteristics except for L. pauciflora from which both fast and slow growers were obtained (Tables 2.2, 2.3). These findings therefore confirm that the taxonomic diversity apparent in the genus Lebeckia (Le Roux \& van Wyk, 2007), is also found amongst the symbiotic rhizobia present in root-nodules of this genus.

The majority of the isolates obtained from the root nodules of the examined Lebeckia species also nodulated cowpea and siratro (Table 2.3). Both are well known to be nodulated by bradyrhizobia (Thies et al., 1991; Mpepereki et al., 1996). Accordingly, slow-growing Lebeckia nodule isolates such as isolates XHR1 (L. simsiana), Betal (L. pungens) and WC21.1 (L. pauciflora) also nodulated and were effective on cowpea, and are therefore likely to be related to 
other nitrogen-fixing cowpea bradyrhizobia. Nodulation of cowpea and siratro is, however, not limited to slow-growing bradyrhizobia. For example, Mpepereki et al. (1996) isolated fastgrowing root nodule bacteria from cowpea in Zimbabwe, and Dakora et al. (2000) published results on fast-growing Rhizobium species from Ghana that effectively nodulate cowpea. Similar findings were obtained in the current study, with the intermediate-growing rhizobial strains from species L. multiflora and L. sericea and the fast-growing Lebeckia rhizobia from L. spinescens, L. ambigua, L. pauciflora and L. meyerianna all able to nodulate cowpea and siratro, often effectively (Table 2.3).

The association of rhizobia with their host legumes is often highly specific. For example, the South African legume Lotononis bainesii forms effective associations only with red-pigmented methylobacteria that cannot nodulate other legumes effectively (Jaftha et al., 2002; Le Roux, 2003). However, the ability of indigenous strains to effectively nodulate various or multiple agricultural legumes has important agronomic implications, mainly because it eliminates the need for commercial inoculant application (Mpepereki et al., 1996). Cowpea, for example, is native to Africa and is effectively nodulated by the indigenous rhizobial populations found in African soils (Mpepereki et al., 1996; Law et al., 2007) as well as in soils of other lands, such as the island of Hawaii (Thies et al., 1991). The initial evaluation of the specificity of the rhizobia isolated from Lebeckia therefore involved inoculation studies of cowpea and siratro, two legumes that are well known for their nodulation compatibility with a wide range of fast and slow-growing rhizobia from other legume species (Thies et al., 1991; Mpepereki et al., 1996; Moulin et al., 2001). Unexpectedly, both intermediate-growing L. cytisoides isolates failed to nodulate either legume although effectively nodulating their host plant, while the L. sepiaria isolate failed to nodulate siratro although effectively nodulating cowpea (Table 2.3). The other strains were all capable of nodulating either legume, although some degree of specificity was observed as nodulation did not always resulting in nitrogen fixation (Table 2.3). Generally, a higher percentage of Lebeckia rhizobia nodulated siratro than cowpea, suggesting that it is more promiscuous than cowpea. The root nodule bacteria of the Lebeckia species are therefore characterized by a variety of nodulation specificities on cowpea and siratro (Table 2.3).

To study nodulation specificity within the Lebeckia symbiotic system, cross-inoculation experiments were performed. However, due to the limited availability of seed supplies for most of the collected species, these tests concentrated only on certain cross-combinations between isolates from the major shrubby and suffrutescent groupings of Lebeckia. Only combinations of 
rhizobia and legumes of the same sections within the shrubby and suffrutescent divisions (Experimental sets 1 and 2), resulted in effective nodulation in each instance (Table 2.4). This suggested that limited specificity might be encountered within each group. For example, cross inoculation between the plants and rhizobia from the sections Stiza and Calobota within the shrubby division resulted in ineffective nodulation. This suggested that the symbiotic nitrogenfixing specificities of the two sections were quite different. Also, combinations of rhizobia and legumes between the shrubby and suffrutescent divisions (Experimental set 3) did not result in nodulation, except for one instance of ineffective nodulation recorded between an isolate from the suffrutescent L. meyerianna inoculated on the shrubby L. multiflora (Table 2.4). This suggested that considerable specificity existed between the two Lebeckia divisions. Similar trends have also been observed in other symbiotic systems. For example, rhizobia from Lotononis angolensis fix nitrogen on their host plant but do not nodulate other species within the Listia section of this genus (L. bainesii, L. listii), whereas reverse inoculation by L. bainesii and L. listii results in ineffective nodulation of L. angolensis (Yates et al., 2007). Taken together, the results of the cross-nodulations tests performed in this study seem to support the idea that nodulation specificity within a specific Lebeckia plant section is much less than that between sections, and that growth habit is an even greater determinant of specificity (Table 2.4). Clarification of this will, however require more exhaustive cross-inoculation tests once sufficient seed is available for this purpose. Further tests may also enable the selection of highly effective broad spectrum strains within each Lebeckia group that may prove useful as specific inoculants for species of this legume and be of commercial benefit.

The findings regarding nodule shape and nodulation presented in this study are in agreement with the taxonomic status of Lebeckia at higher order and sub generic levels. Nodule shape has long been recognized as an important taxonomic criterion in legume systematics (Sprent, 2002). Like other legumes in the tribe Crotalarieae, Lebeckia has indeterminate so-called "crotalarioid" nodules (Fig. 2.4) that are in most cases globular consisting of many lobes resulting in a fanshape (Corby et al., 1983; Sprent \& James, 2007). The Crotalarieae share this property with other genistoid tribes (e.g. Genisteae, Sophoreae and Podalyrieae) with which it also shares a common ancestor (Lavin et al.; 2005). In contrast, inoculation of the Lebeckia rhizobia onto the phaseoloid legumes cowpea and siratro resulted in formation of typical "desmodioid" nodules (Sprent, 2002) that are a feature common among the millettioid crown clade (i.e. tribes Phaseoleae, Desmodieae and Psoraleae) (Lavin et al., 2005). This illustrated the dominance of the host plant in determining nodule shape (Sprent, 2007). At the sub generic level, the results of 
my cross-inoculation studies support the plant sections proposed for Lebeckia by van Wyk and co-workers (Le Roux \& van Wyk, 2007). Rhizobia were only able to effectively nodulate other members within the same section, while rhizobia from one growth habit (shrubby trifoliate leaf) did not nodulate suffrutescent needle leaf plants in the other habit (Table 2.4). This supports the idea that the growth habit of Lebeckia is a useful taxonomic criterion for division of this genus into two genera (Boatwright and Van Wyk, personal communication).

This is the first report on the symbiotic properties of rhizobia of Lebeckia species indigenous to South Africa. The results showed that Lebeckia is nodulated by diverse rhizobia including fast-, slow- and intermediate-growers. Some of these may potentially be used as effective commercial inoculants if they can out-compete natural soil populations found in South Africa. As shown in this study, the symbiotic nitrogen-fixation effectiveness of indigenous rhizobia on siratro and cowpea were similar and may resemble the symbiotic capabilities of effective cowpea rhizobia found elsewhere in southern Africa (Law et al., 2007). In some instances these indigenous bacteria are more effective on cowpea than the inoculant strain CB756 (Law et al., 2007). However, many of the Lebeckia rhizobia included in this study appear to be specific to their homologous hosts and many did not effectively nodulate cowpea. As the Lebeckia species examined in this study were representative of the largest and most common sections in this genus, the use of Lebeckia rhizobia in broad spectrum inoculants for Lebeckia and other legumes is likely to be negligible considering the evidence presented in this study. Their potential usefulness may be restricted to nursery plantings only. 


\subsection{REFERENCES}

Bamarouf, A., Eley, A., \& Winstanley, T. 1996. Evaluation of methods for distinguishing Gram-positive from Gram-negative anaerobic bacteria. Anaerobe. 2: 163-168.

Barrett, C. F., \& Parker, M. A. 2006. Coexistence of Burkholderia, Cupriavidus and Rhizobium sp. nodule bacteria on two Mimosa spp. in Costa Rica. Appl. Environ. Microbiol. 72: 1198-1206.

Bentham, G. 1844. Enumeration of Leguminous indigenous to southern Asia and central and southern Africa. Hook. Lond. J. Bot. 3: 338-365.

Boatwright, J. S., van Wyk, B. - E., \& Tilney, P. M. 2007. Systematic studies in the genus Lebeckia and related genera: A revision of Lebeckia section Stiza (Crotalarieae, Fabaceae). S. Afr. J. Bot. 73: 280

Boatwright, J. S., \&, van Wyk, B. - E. 2007. The identity of Lebeckia lotononoides (Crotalarieae, Fabaceae). S. Afr. J. Bot. 73: 664-666.

Corby, H.D.L., Polhill, R. M., \& Sprent, J. I. 1983. Taxonomy. In: Broughton, W. J. (Ed). Nitrogen fixation, Legumes, Vol. 3. Oxford University Pp 1-35.

Dakora, F. D. 2000. Commonality of root nodulation signals and nitrogen assimilation in tropical grain legumes belonging to the tribe Phaseoleae. Aust. J. Plant Physiol. 27: 885-892.

Germishuizen, G., \& Meyer, N. L. 2003. Plants of Southern Africa, an annotated checklist. Strelitzia 14. National Botanical Institute, Pretoria.

Goszczynska, T., Serfontein, J. J., \& Serfontein, S. 2000. Introduction to practical phytobacteriology. A manual for Phytobacteriology. ARC-Plant Protection Research Institute, Safrinet.

Halebian, S., Harris, B., Finegold, S. M., \& Rolfe, R. D. 1981. Rapid methods that aid in distinguishing Gram-positive from Gram-negative anaerobic bacteria. J. Clin. Microbiol. 13: 444-448. 
Harvey, W. H. 1862. Leguminosae. In: Harvey, W. H., Sonder, O. W. (Eds). Flora Capensis, Vol. 2. Hodges, Smith \& Co., Dublin. Pp 83-86

Jaftha, J. B., Strijdom, B. W., \& Steyn, P. L. 2002. Characterization of pigmented Methylotrophic bacteria which nodulate Lotononis bainesii. Syst. Appl. Microbiol. 25: 440-449.

Lavin, M., Herendeen, P. S., \& Wojciechowski, M. F. 2005. Evolutionary rates analysis of Leguminosae implicates rapid diversification of lineages during the tertiary. Syst. Biol. 54: 574594.

Law, I. J., Botha, W. J., Majaule, U. C., \& Phalane, F. L. 2007. Symbiotic and genomic diversity of "cowpea" bradyrhizobia from soils in Botswana and South Africa. Biol. Fertil. Soils. 43: 653-663.

Le Roux, A., \& Schelpe, T. 1997. Namaqualand. South African wildflower guide 1. Botanical Society of South Africa, Kirstenbosch.

Le Roux, J. J. 2003. The diversity of root nodule bacteria associated with indigenous Lotononis spp. as determined by sodium dodecyl-sulphate polyacrylamide gel electrophoresis and 16S rDNA sequencing. MSc. thesis. University of Pretoria, Pretoria, Republic of South Africa.

Le Roux, M. M., \& van Wyk, B. - E. 2007. A revision of Lebeckia sect. Lebeckia: The L. sepiaria group. S. Afr. J. Bot. 73: 118-130.

Le Roux, M. M., van Wyk, B. - E., \& van der Bank, M. 2007. A taxonomic study of the type section of the genus Lebeckia Thunb (Fabaceae, Crotalarieae). S. Afr. J. Bot. 73: 297.

Le Roux, P. M., Kotze, G. P., \& Glen, H. F. 1994. Bossieveld. Grazing plants of the Karoo and karoo-like areas. Bulletin 428. Department of Agriculture, Pretoria.

Moulin, L., Munive, A., Dreyfus, B., \& Boivin-Masson, C. 2001. Nodulation of legumes by members of the $\beta$-subclass of Proteobacteria. Nature. 411: 948-950. 
Mpepereki S., Wollum, I. I., \& Makonese, F. 1996. Diversity in symbiotic specificity of cowpea rhizobia indigenous to Zimbabwean soils. Plant Soil. 186: 167-171.

Preston, N. W., \& Morrell, A. 1962. Reproducible results with the Gram stain. J. Path. Bacteriol. 84: 241-243.

Shearing, D., \& van Heerden, K. 1994. Karoo. South African wild flower guide 6. Botanical Society of South Africa, Kirstenbosch.

Somasegaran, P., \& Hoben, H. J. 1994. Hand book for rhizobia. Springer-Verlag, Heidelberg.

Sprent, J. I. 2002. Nodulation as a taxonomic tool. In: Herendeen, P.S. \& Bruneau, A. (Eds). Advances in legume systematics, part 9, Royal Botanical Gardens, Kew. Pp 21-44.

Sprent, J. I. 2007. Evolving ideas of legume evolution and diversity: a taxonomic perspective on the occurrence of nodulation. New Phytol. 174: 11-25.

Sprent, J. I., \& James, E. K. 2007. Legume evolution: Where do nodules and mycorrhizas fit in. Update on legume evolution. Plant Physiol. 144: 575-581.

Thies, J. E., Bohlool, B. B., \& Singleton, P. W. 1991. Subgroups of the Cowpea miscellany: Symbiotic Specificity within Bradyrhizobium spp. for Vigna unguiculata, Phaseolus lunatus, Arachis hypogaea and Macroptilium atropurpureum. Appl. Environ. Microbiol. 57: 1540-1545.

Van Breda, P. A. B, \& Barnard, S. A. 1991. 100 veld plants of the winter rainfall region. A guide to the use of veld plants for grazing. Bulletin No. 422. Department of Agricultural Development, Government press, Pretoria.

Yates, R. J., Howieson, J. G., Reeve, W. G., Nandasena, K. G., Law, I. J., Brau, L., Ardley, J. K., Nistelberger, H. M., Real, D., \& O'Hara, G. W. 2007. Lotononis angolensis forms nitrogen fixing, lupinoid nodules with phylogenetically unique, fast-growing, pink-pigmented bacteria, which do not nodulate L. bainesii or L listii. Soil Biol. Biochem. 39: 1680-1688. 


\subsection{TABLES}

Table 2.1. Coordinates and soil properties for the collection sites of the ten Lebeckia species examined in this study.

\begin{tabular}{llcccc}
\hline Lebeckia species & \multicolumn{1}{c}{ Locality } & Latitude & Longitude & pH & Soil type \\
\hline L. cytisoides & Citrusdal & -32.2999 & 18.5990 & 5.25 & Stony clay loam \\
L. multiflora & Lambert's bay & -32.0356 & 18.1896 & 6.50 & Sandy \\
L. sericea & Bitterfontein & -30.5877 & 18.1466 & 6.50 & Sandy loam \\
L. spinescens & Beaufort West & -32.1416 & 22.5519 & 6.50 & Sandy \\
L. pungens & Meiringspoort & -33.1979 & 22.3246 & 6.00 & Sandy loam \\
L. ambigua & Modder river & -33.2921 & 18.1936 & 6.70 & Sandy \\
L. sepiaria & Citrusdal & -32.2999 & 18.5990 & 5.25 & Stony clay loam \\
L. pauciflora & Cedarberg & -32.2185 & 19.0247 & 6.00 & Sandy loam \\
L. meyerianna & Clanwilliam & -32.0187 & 18.4742 & 6.00 & Sandy \\
L. simsiana & Stellenbosch & -33.9333 & 18.8500 & 6.00 & Sandy \\
\hline
\end{tabular}


Table 2.2. Strains and geographic origin in South Africa of rhizobia isolated from ten Lebeckia species within three sections with either a shrubby or a suffrutescent habit.

\begin{tabular}{|c|c|c|c|c|c|}
\hline $\begin{array}{c}\text { Strain } \\
\text { number }\end{array}$ & Growth rate a $^{a}$ & Host species & $\begin{array}{c}\text { Lebeckia } \\
\text { Section }\end{array}$ & Habit & Geographic origin \\
\hline BW1LSab & $\mathrm{F}$ & L. spinescens & Calobota & Shrubby & Beaufort West \\
\hline BW2LSa & $"$ & $"$ & $"$ & " & $"$ \\
\hline BW2LSb & $"$ & $"$ & $"$ & $"$ & " \\
\hline BWS1iii & $"$ & $"$ & $"$ & $"$ & $"$ \\
\hline BW3LSc & $"$ & $"$ & $"$ & $"$ & $"$ \\
\hline BW3LSd & $"$ & $"$ & $"$ & $"$ & $"$ \\
\hline BHLSb & $"$ & $"$ & $"$ & $"$ & $"$ \\
\hline BH1LSa & $"$ & $"$ & $"$ & $"$ & $"$ \\
\hline BH1LSb & $"$ & $"$ & $"$ & " & $"$ \\
\hline BH1LSc & $"$ & $"$ & $"$ & $"$ & " \\
\hline BH2LSa & $"$ & $"$ & $"$ & $"$ & " \\
\hline BH2LSb & $"$ & $"$ & $"$ & $"$ & " \\
\hline BH2LSc & $"$ & $"$ & $"$ & $"$ & $"$ \\
\hline BH3LSa & $"$ & $"$ & $"$ & " & " \\
\hline BH3LSb & $"$ & $"$ & $"$ & $"$ & $"$ \\
\hline BH3LSc & $"$ & $"$ & $"$ & $"$ & $"$ \\
\hline WC19.1a & $\mathrm{M}$ & L. cytisoides & $"$ & " & Citrusdal \\
\hline WC19.1b & $"$ & " & $"$ & $"$ & - 1t \\
\hline WC23.1a & $\mathrm{M}$ & L. multiflora & $"$ & $"$ & Lamberts bay \\
\hline WC $23.1 \mathrm{~b}$ & $"$ & с. mont & $"$ & $"$ & " \\
\hline WC23.1c & $"$ & $"$ & $"$ & " & " \\
\hline WC23.1d & $"$ & $"$ & $"$ & $"$ & $"$ \\
\hline NK22 & $\mathrm{M}$ & L. sericea & $"$ & $"$ & Kamiesberg \\
\hline WC28.1a & $"$ & 2. sect & $"$ & $"$ & Bitterfontein \\
\hline WC $28.1 \mathrm{~b}$ & $"$ & $"$ & $"$ & $"$ & " \\
\hline WC $28.1 \mathrm{c}$ & $"$ & $"$ & $"$ & $"$ & " \\
\hline WC28.1e & $"$ & $"$ & $"$ & $"$ & " \\
\hline WC28.1f & $"$ & $"$ & $"$ & $"$ & $"$ \\
\hline WC28.1g & $"$ & $"$ & $"$ & " & " \\
\hline WC28.1h & $"$ & $"$ & $"$ & $"$ & " \\
\hline WC $28.1 \mathrm{i}$ & $"$ & $"$ & $"$ & $"$ & $"$ \\
\hline WC $28.1 \mathrm{j}$ & $"$ & $"$ & $"$ & " & " \\
\hline WC 33a & " & $"$ & $"$ & " & " \\
\hline WC $33 b$ & $"$ & $"$ & $"$ & $"$ & $"$ \\
\hline WC $33 \mathrm{c}$ & $"$ & $"$ & $"$ & $"$ & " \\
\hline WC33d & $"$ & $"$ & $"$ & $"$ & " \\
\hline WC $33 \mathrm{e}$ & $"$ & $"$ & $"$ & $"$ & $"$ \\
\hline WC $33 \mathrm{f}$ & $"$ & $"$ & $"$ & $"$ & " \\
\hline WC $33 \mathrm{~g}$ & $"$ & $"$ & $"$ & $"$ & $"$ \\
\hline WC $33 \mathrm{~h}$ & $"$ & $"$ & " & " & " \\
\hline Betal106a & VS & L. pungens & Stiza & $"$ & Meiringspoort \\
\hline Betal106b & $"$ & " P. Pons & " & $"$ & \\
\hline Betal106c & $"$ & $"$ & $"$ & " & $"$ \\
\hline Betal106d & $"$ & $"$ & $"$ & $"$ & " \\
\hline Betal106e & $"$ & $"$ & $"$ & $"$ & $"$ \\
\hline Betal106f & $"$ & $"$ & $"$ & $"$ & $"$ \\
\hline Betal106g & $"$ & $"$ & $"$ & $"$ & $"$ \\
\hline Betal106h & $"$ & $"$ & $"$ & $"$ & " \\
\hline Betal106i & $"$ & $"$ & $"$ & " & $"$ \\
\hline Betal106j & $"$ & $"$ & $"$ & $"$ & $"$ \\
\hline WC5.4a & $\mathrm{F}$ & L. ambigua & Lebeckia & Suffrutescent & Modder Rivier \\
\hline WC $5.4 \mathrm{~b}$ & & & & & \\
\hline WC5.4c & $"$ & $"$ & $"$ & $"$ & $"$ \\
\hline WC5.4d & $"$ & $"$ & $"$ & $"$ & $"$ \\
\hline WC5.4e & $"$ & $"$ & $"$ & $"$ & $"$ \\
\hline WC5.4f & $"$ & $"$ & $"$ & " & " \\
\hline WC5.4g & $"$ & $"$ & $"$ & $"$ & $"$ \\
\hline WC5.4h & $"$ & $"$ & $"$ & $"$ & $"$ \\
\hline WC5.4i & $"$ & $"$ & $"$ & " & $"$ \\
\hline WC12.1a & $\mathrm{S}$ & L. sepiaria & $"$ & $"$ & Citrusdal \\
\hline WC21.1a & $\mathrm{F}$ & L. pauciflora & $"$ & $"$ & Cedarberg \\
\hline WC21.1b & $"$ & 5. p & $"$ & $"$ & " \\
\hline WC21.1c & $"$ & $"$ & $"$ & $"$ & $"$ \\
\hline WC21.1d & $"$ & $"$ & $"$ & $"$ & " \\
\hline WC21.1e & $"$ & $"$ & $"$ & $"$ & $"$ \\
\hline WC21.1f & $"$ & $"$ & $"$ & $"$ & " \\
\hline WC21.1g & $"$ & $"$ & $"$ & $"$ & " \\
\hline WC21.1h & $"$ & $"$ & $"$ & " & " \\
\hline WC21.1i & $"$ & $"$ & $"$ & $"$ & $"$ \\
\hline WC $21.1 j$ & $"$ & $"$ & $"$ & " & $"$ \\
\hline WC $21.1 \mathrm{k}$ & $\mathrm{S}$ & $"$ & $"$ & $"$ & $"$ \\
\hline WC21.11 & $"$ & $"$ & $"$ & " & " \\
\hline WC $26.1 \mathrm{c}$ & $\mathrm{F}$ & L. meyerianna & $"$ & $"$ & Clanwilliam \\
\hline WC26.1d & $"$ & & $"$ & $"$ & \\
\hline WC26.1e & $"$ & $"$ & $"$ & $"$ & $"$ \\
\hline WC26.1e1 & $"$ & $"$ & $"$ & $"$ & " \\
\hline WC26.1f & $"$ & $"$ & $"$ & $"$ & $"$ \\
\hline XHR1a & $\mathrm{S}$ & L. simsiana & $"$ & $"$ & Stellenbosch \\
\hline XHR1b & & & $"$ & $"$ & \\
\hline
\end{tabular}

a F, fast; M, intermediate; S, slow; VS, very slow 
Table 2.3. Colony growth and nodulation characteristics of the rhizobia isolated from Lebeckia species used in this study.

\begin{tabular}{|c|c|c|c|c|c|c|}
\hline \multirow{2}{*}{$\begin{array}{l}\text { Lebeckia } \\
\text { species }\end{array}$} & \multirow{2}{*}{$\begin{array}{c}\text { Lebeckia } \\
\text { section }\end{array}$} & \multirow{2}{*}{$\begin{array}{c}\text { Number } \\
\text { of } \\
\text { isolates }\end{array}$} & \multirow[t]{2}{*}{ Growth rate $^{a}$} & \multicolumn{3}{|c|}{ Number of isolates that nodulated ${ }^{b}$} \\
\hline & & & & Lebeckia & Cowpea & Siratro \\
\hline L. cytisoides & Calobota & 2 & Intermediate; 5-6d, $1 \mathrm{~mm}$ & $2 \mathrm{E} ; 100 \%$ & $\mathrm{~N}$ & $\mathrm{~N}$ \\
\hline L. multiflora & $"$ & 4 & Intermediate; $5-6 \mathrm{~d}, 1 \mathrm{~mm}$ & $4 \mathrm{E} ; 100 \%$ & $2 \mathrm{E}, 2 \mathrm{I} ; 50 \%$ & $4 \mathrm{E} ; 100 \%$ \\
\hline L. sericea & $"$ & 18 & Intermediate; $5-6 \mathrm{~d}, 1-2 \mathrm{~mm}$ & $18 \mathrm{E} ; 100 \%$ & 9E, 9I; $50 \%$ & $12 \mathrm{E}, 6 \mathrm{I} ; 66 \%$ \\
\hline L. spinescens & $"$ & 16 & Fast, $3 \mathrm{~d} ; \geq 3 \mathrm{~mm}$ & $16 \mathrm{E} ; 100 \%$ & $2 \mathrm{E}, 14 \mathrm{I}, 12 \%$ & $12 \mathrm{E}, 4 \mathrm{I} ; 75 \%$ \\
\hline L. pungens & Stiza & 10 & Very slow; $8 \mathrm{~d}, \leq 1 \mathrm{~mm}$ & $10 \mathrm{E} ; 100 \%$ & $8 \mathrm{E}, 2 \mathrm{I} ; 80 \%$ & $10 \mathrm{E} ; 100 \%$ \\
\hline L. ambigua & Lebeckia & 9 & Fast; 3-4d, 2mm & $9 \mathrm{E} ; 100 \%$ & $6 \mathrm{E}, 3 \mathrm{I} ; 67 \%$ & $9 \mathrm{E} ; 100 \%$ \\
\hline L. sepiaria & $"$ & 1 & Slow; 6d, 1mm & $1 \mathrm{E} ; 100 \%$ & $1 \mathrm{E} ; 100 \%$ & $\mathrm{~N}$ \\
\hline L. pauciflora & $"$ & 10 & Fast; $3-4 \mathrm{~d}, 2 \mathrm{~mm}$ & $10 \mathrm{E} ; 100 \%$ & $8 \mathrm{E}, 2 \mathrm{I} ; 80 \%$ & $6 \mathrm{E}, 4 \mathrm{I} ; 60 \%$ \\
\hline " & $"$ & 2 & Slow; $6 \mathrm{~d}, 1 \mathrm{~mm}$ & $2 \mathrm{E} ; 100 \%$ & $2 \mathrm{E} ; 100 \%$ & $2 \mathrm{E} ; 100 \%$ \\
\hline L. meyerianna & $"$ & 5 & Fast; $3-4 \mathrm{~d}, 2 \mathrm{~mm}$ & $5 \mathrm{E} ; 100 \%$ & $4 \mathrm{E}, 1 \mathrm{I} ; \quad 80 \%$ & $4 \mathrm{E}, 1 \mathrm{I} ; 80 \%$ \\
\hline L. simsiana & $"$ & 2 & Very slow; $8 \mathrm{~d},<1 \mathrm{~mm}$ & $2 \mathrm{E} ; 100 \%$ & $2 \mathrm{E} ; 100 \%$ & $2 \mathrm{E} ; 100 \%$ \\
\hline
\end{tabular}

a Relative growth rates (very slow to fast) are indicated in terms of colony diameters $(\mathrm{mm})$ reached after a specific number of days (d) incubation

b Columns show nodulated plant numbers and percentage effective nodulation of the respective rhizobia on their homologous hosts as well as cowpea and siratro. E, effective nodules; I, ineffective nodules; N, no nodulation 
Table 2.4. Nodulation effectiveness of rhizobial strains isolated from shrubby and suffrutescent Lebeckia species after cross-inoculation on these species.

\begin{tabular}{|c|c|c|c|c|c|}
\hline $\begin{array}{c}\begin{array}{c}\text { Cross-inoculation } \\
\text { sets }^{\mathrm{a}}\end{array} \\
\end{array}$ & Strain & Original host & Inoculated species & $\begin{array}{c}\text { Sections cross } \\
\text { inoculated }\end{array}$ & Nodulation $^{b}$ \\
\hline \multirow[t]{14}{*}{ 1: Shrubby } & WC19.1b & L. cytisoides & L. multiflora & Calobota $\rightarrow$ Calobota & $\mathrm{E}$ \\
\hline & WC19.1b & L. cytisoides & L. sericea & $"$ & $\mathrm{E}$ \\
\hline & WC23.1b & L. multiflora & L. cytisoides & $"$ & $\mathrm{E}$ \\
\hline & WC33b & L. sericea & L. cytisoides & $"$ & $\mathrm{E}$ \\
\hline & Betal106a & L. pungens & L. spinescens & Stiza $\rightarrow$ Calobota & I \\
\hline & Betal106f & L. pungens & L. spinescens & " & I \\
\hline & Betal106a & L. pungens & L. sericea & $"$ & I \\
\hline & Betal106f & L. pungens & L. cytisoides & $"$ & I \\
\hline & Betal106f & L. pungens & L. multiflora & $"$ & I \\
\hline & WC28.1f & L. sericea & L. pungens & Calobota $\rightarrow$ Stiza & I \\
\hline & WC19.1c & L. cytisoides & L. pungens & " & I \\
\hline & BH1LSc & L. spinescens & L. pungens & $"$ & I \\
\hline & BW3LSd & L. spinescens & L. pungens & $"$ & I \\
\hline & WC23.1b & L. multiflora & L. pungens & $"$ & $\mathrm{I}$ \\
\hline \multirow[t]{8}{*}{ 2: Suffrutescent } & WC5.4d & L. ambigua & L. meyerianna & Stiza $\rightarrow$ Lebeckia & $\mathrm{E}$ \\
\hline & WC5.4e & L. ambigua & L. meyerianna & $"$ & $\mathrm{E}$ \\
\hline & WC26.1d & L. meyerianna & L. ambigua & Calobota $\rightarrow$ Lebeckia & $\mathrm{E}$ \\
\hline & WC26.1f & L. meyerianna & L. ambigua & $"$ & $\mathrm{E}$ \\
\hline & WC26.1f & L. meyerianna & L. simsiana & $"$ & $\mathrm{E}$ \\
\hline & WC26.1d & L. meyerianna & L. simsiana & $"$ & $\mathrm{E}$ \\
\hline & XHR1a & L. simsiana & L. meyerianna & $"$ & $\mathrm{E}$ \\
\hline & XHR1b & L. simsiana & L. meyerianna & $"$ & $\mathrm{E}$ \\
\hline \multirow[t]{14}{*}{ 3: Mixed } & Betal106d & L. pungens & L. meyerianna & Calobota $\rightarrow$ Lebeckia & $\mathrm{N}$ \\
\hline & Betal106a & L. pungens & L. meyerianna & $"$ & $\mathrm{~N}$ \\
\hline & WC19.1b & L. cytisoides & L. meyerianna & $"$ & $\mathrm{~N}$ \\
\hline & WC23.1b & L. multiflora & L. ambigua & $"$ & $\mathrm{~N}$ \\
\hline & WC33a & L. sericea & L. ambigua & $"$ & $\mathrm{~N}$ \\
\hline & WC26.1f & L. meyerianna & L. pungens & Lebeckia $\rightarrow$ Stiza & $\mathrm{N}$ \\
\hline & WC26.1e & L. meyerianna & L. pungens & $"$ & $\mathrm{I}$ \\
\hline & WC26.1e & L. meyerianna & L. cytisoides & Lebeckia $\rightarrow$ Calobota & $\mathrm{N}$ \\
\hline & WC26.1e & L. meyerianna & L. spinescens & " & $\mathrm{N}$ \\
\hline & WC26.1c & L. meyerianna & L. spinescens & $"$ & $\mathrm{~N}$ \\
\hline & WC26.1d & L. meyerianna & L. cytisoides & $"$ & $\mathrm{~N}$ \\
\hline & WC26.1c & L. meyerianna & L. cytisoides & $"$ & $\mathrm{~N}$ \\
\hline & WC5.4d & L. ambigua & L. sericea & $"$ & $\mathrm{~N}$ \\
\hline & WC5.4d & L. ambigua & L. multiflora & $"$ & $\mathrm{~N}$ \\
\hline
\end{tabular}

a Set 1, Rhizobia isolated from shrubby Lebeckia species were used to inoculate other shrubby species. Set 2, Rhizobia from suffrutescent species were used to crossinoculate other suffrutescent species. Set 3, Rhizobia from shrubby Lebeckia species were used to inoculate suffrutescent species and vice versa

b Nodulation by the respective rhizobia are indicated as follows. E, effective nodules; $\mathrm{I}$, ineffective nodules; $\mathrm{N}$, no nodulation 


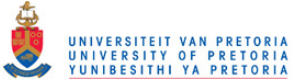

\subsection{FIGURES}

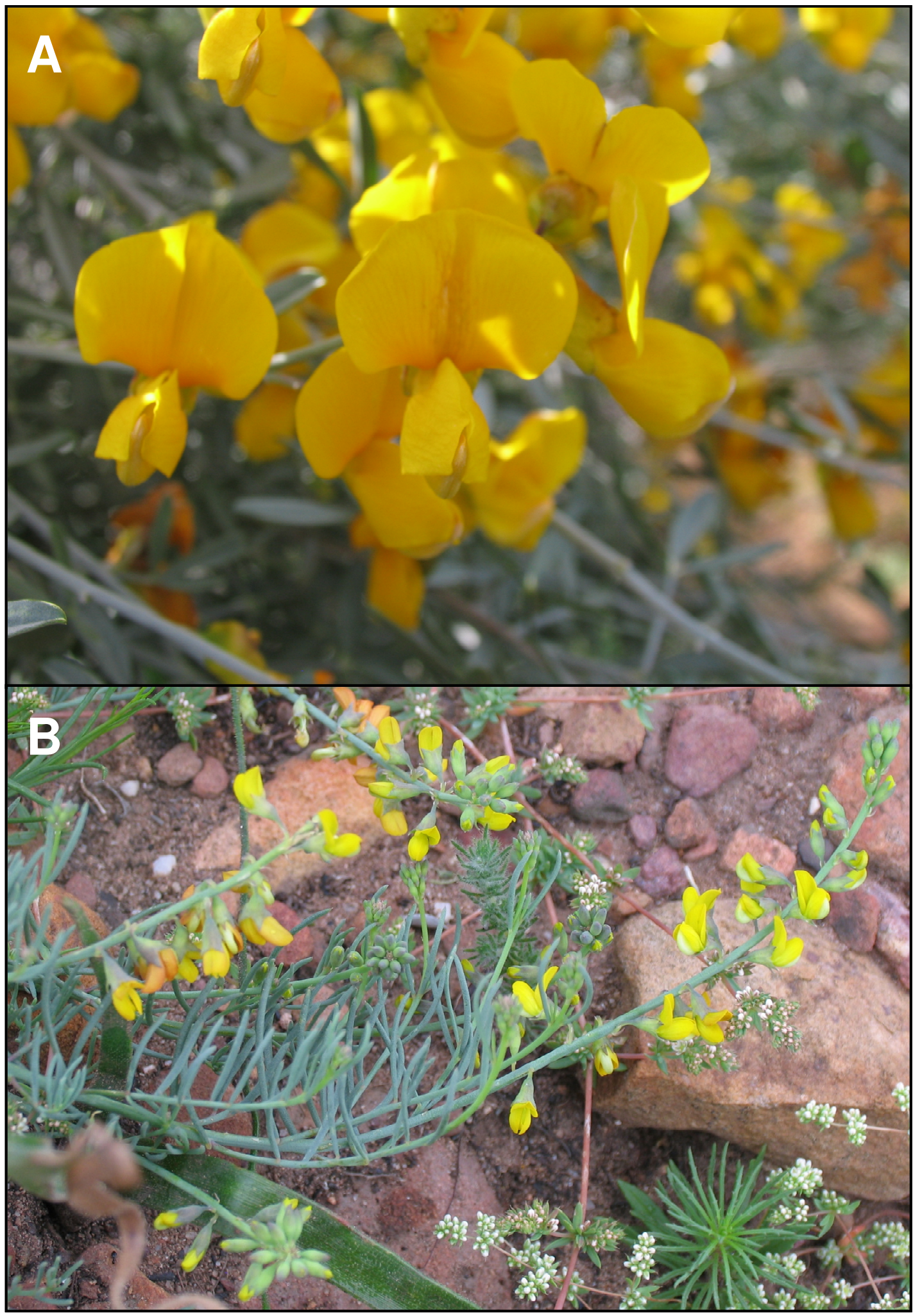


Figure 2.1. Flower spikes of the shrubby Lebeckia cytisoides (A) and the suffrutescent herbaceous L. plukenetiana (B) flowering during the spring season in the Western Cape. Photos courtesy of J. S. Boatwright 


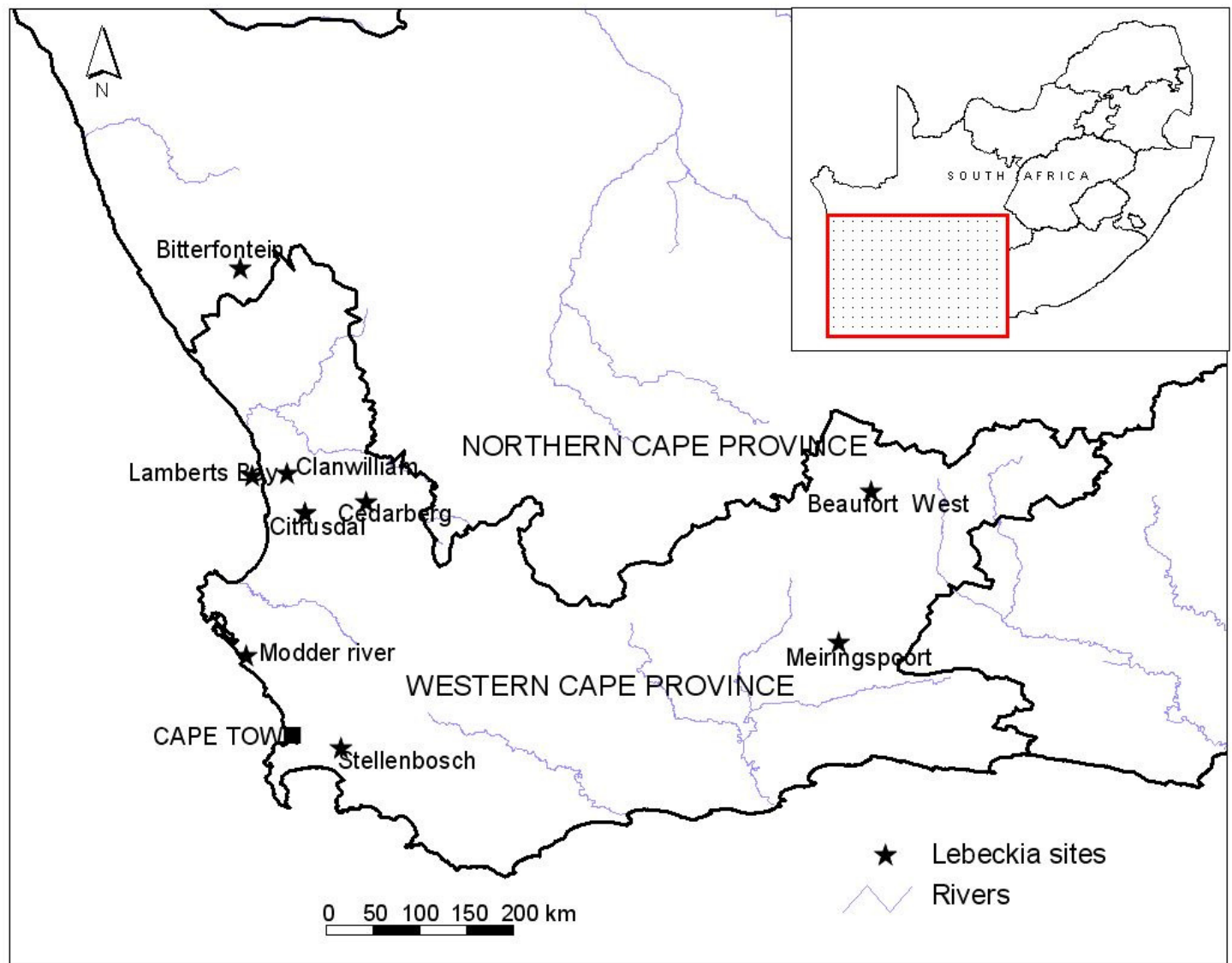

Figure 2.2. A map of South Africa showing the Lebeckia collection sites indicated with stars and rivers with stippled lines, in the Western and Northern Cape provinces. 


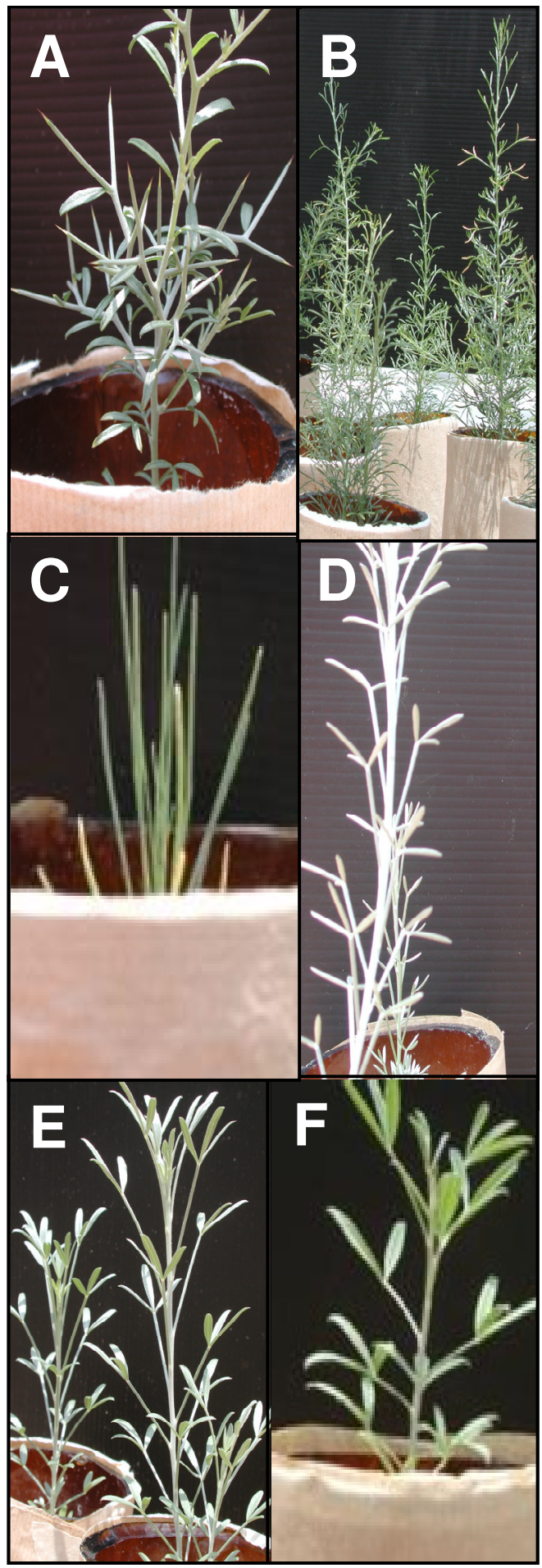

Figure 2.3. L. ambigua (C) with needle-shaped leaves (habit, suffrutescent) and the trifoliateleaf species (habit, shrubby) Lebeckia pungens (A), L. spinescens (B), L. multiflora (D) L. sericea $(\mathrm{E})$ and $L$. cytisoides $(F)$ growing in nitrogen-free Leonard jars and effectively fixing nitrogen as indicated by the dark green colour of the leaves. 


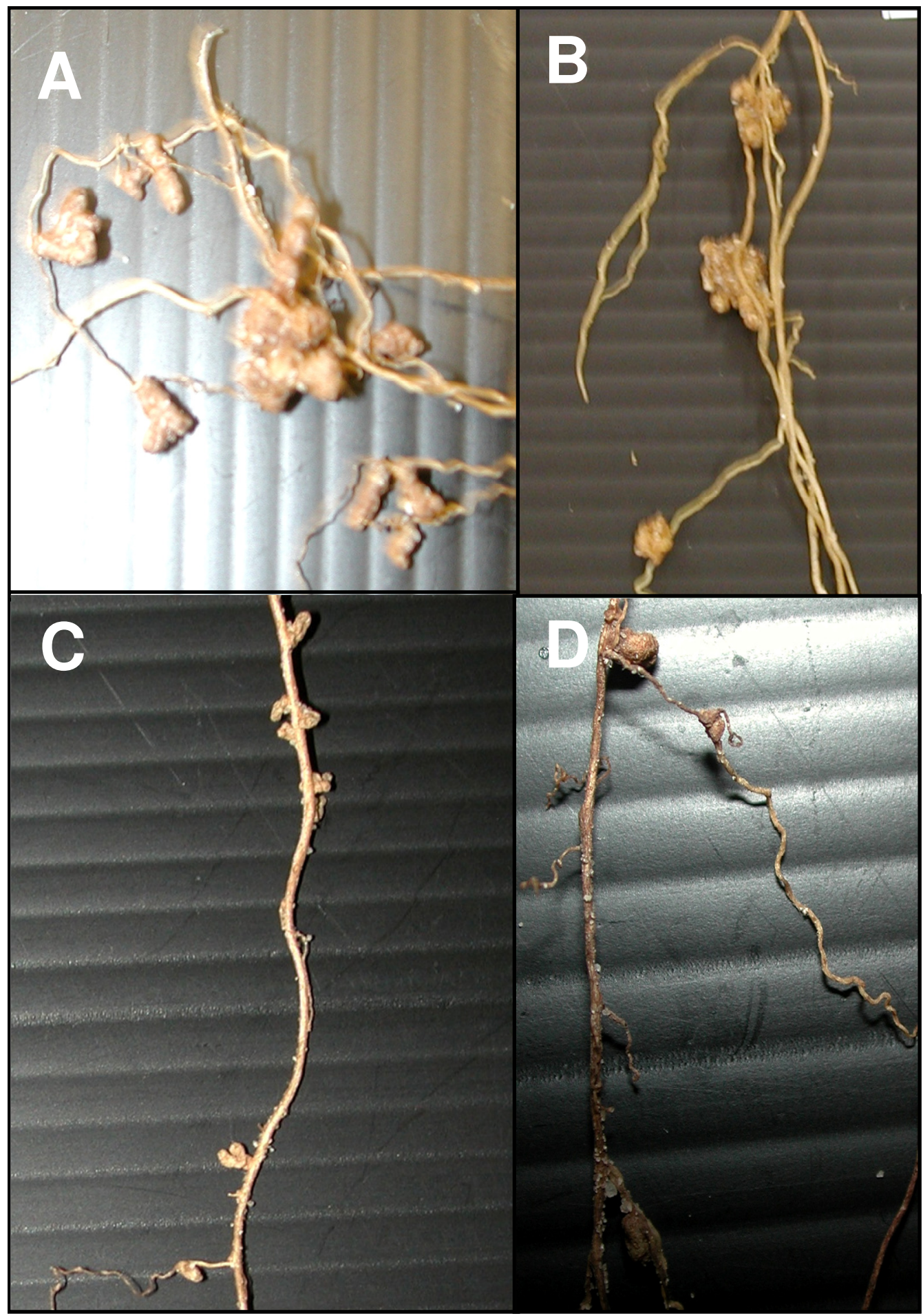

Figure 2.4. Examples of the branched, globular and elongated indeterminate nodules on the roots of Lebeckia species (A and B) and ovate to lobed indeterminate nodules on siratro roots (C) and spherical determinate nodules on cowpea roots (D). 
CHAPTER 3

DNA FINGERPRINTING AND 16S rRNA GENE ANALYSIS OF RHIZOBIA ASSOCIATED WITH LEBECKIA SPECIES IN SOUTH AFRICA

\section{TABLE OF CONTENTS}

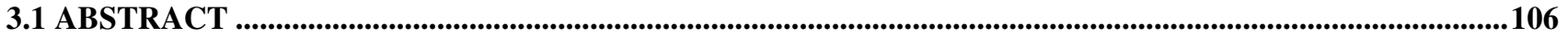

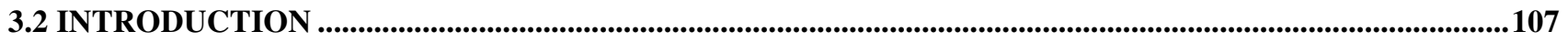

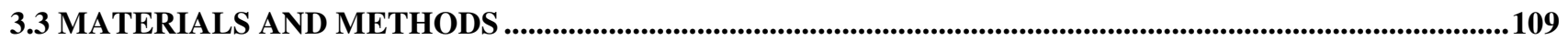

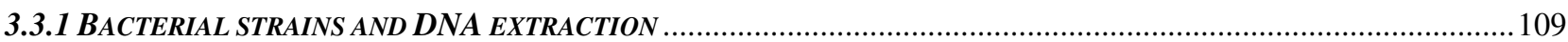

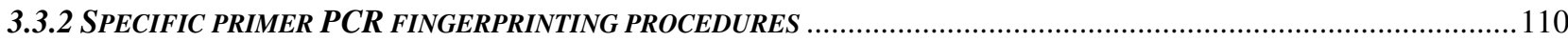

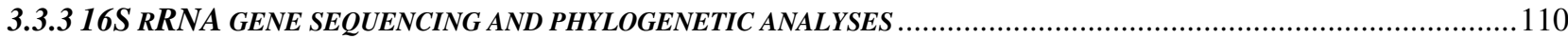

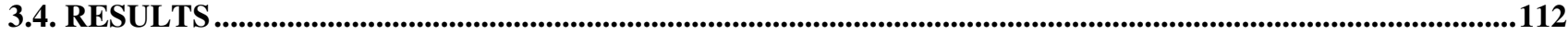

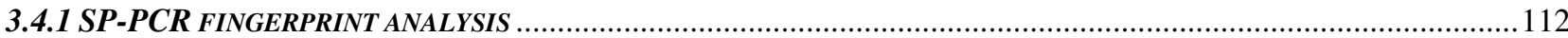

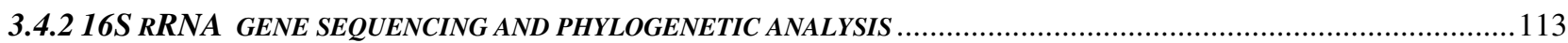

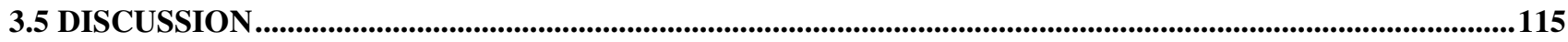

3.6 REFERENCES …..........................................................................................................................................121

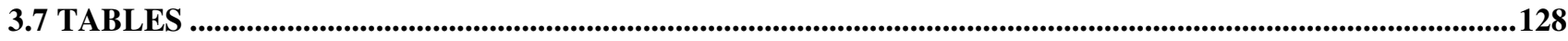

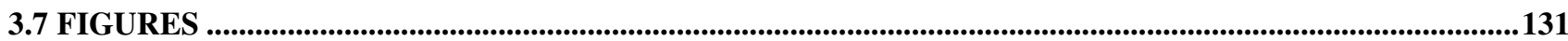




\subsection{ABSTRACT}

Diversity among 79 nodule isolates from shrubby and suffrutescent Lebeckia was assessed using random amplified DNA fingerprints. Cluster analyses indicated that isolates from the same species of Lebeckia generally grouped together in individual clusters with $\geq 65 \%$ similarity. The gene encoding the $16 \mathrm{~S}$ ribosomal RNA subunit was sequenced in 39 isolates representing the various clusters. The resulting sequences were compared to those in public domain nucleotide databases and subjected to phylogenetic analyses. The respective $16 \mathrm{~S}$ sequences were most closely related to members of the $\alpha$-proteobacterial genera Bradyrhizobium, Sinorhizobium and Mesorhizobium, as well as the $\beta$-proteobacterial genus, Burkholderia. These results indicate that indigenous South African Lebeckia species are nodulated by diverse rhizobia.

Keywords: Lebeckia, diversity, 16S rRNA, Bradyrhizobium, Sinorhizobium Mesorhizobium and Burkholderia. 


\subsection{INTRODUCTION}

The nitrogen-fixing symbiotic bacteria of several legume genera within the tribe Crotalarieae have been studied taxonomically (Sy et al., 2001; Jaftha et al., 2002). These include agriculturally and economically important species of Crotalaria, Lotononis and Aspalathus (Sy et al., 2001; Moulin et al., 2001; Jaftha et al., 2002; Le Roux, 2003; Yates et al., 2007). Plants of the genus Crotalaria are used for green manures and intercropping (Giller, 2001), Lotononis bainesii is a valuable forage legume (Yates et al., 2007) and Aspalathus linearis is the source of the well-known rooibos tea beverage (van Heerden et al., 2003). Dagutat (1995) carried out a systematic investigation into the identity of root nodule bacteria or rhizobia associated with a multiplicity of legumes found in South Africa, but neither she nor several later workers included Lebeckia spp. in their studies (Dagutat, 1995; Jaftha et al., 2002; Le Roux, 2003; Lindique, 2005).

Previously, 79 isolates of root nodule bacteria (rhizobia) from 10 species of Lebeckia were isolated and characterised in terms of their culture and nodulation properties (Chapter 2 of this dissertation). This was to complement a taxonomic revision of Lebeckia being undertaken at the University of Johannesburg, Johannesburg (Le Roux \& van Wyk, 2007; van Wyk, personal communication). The results of this previous study indicated that Lebeckia is nodulated by diverse rhizobia including fast-, slow- and intermediate-growers. Some of these may potentially be used as effective inoculants for improving nitrogen fixation in commercial legumes. Inoculation studies also indicated that only combinations of rhizobia and legumes from the same sections within the shrubby and suffrutescent divisions of Lebeckia resulted in effective nodulation in each instance, suggesting that limited specificity might be encountered within each group. For example, cross-inoculation between the plants and rhizobia from the sections Stiza and Calobota within the shrubby division resulted in ineffective nodulation, suggesting that the symbiotic specificities of the two sections were quite different. Also, combinations of rhizobia and legumes between the shrubby and suffrutescent divisions generally did not result in nodulation, suggesting that considerable nodulation specificity existed between the two Lebeckia divisions.

The aim of the present study was to determine the taxonomic identities of the rhizobia obtained from the Lebeckia species found at nine localities in South Africa (Chapter 2 of this dissertation). For this 
purpose, relatedness among the isolates was initially screened using specific primer (SP) PCR fingerprinting (Law et al., 2007). From the resulting clusters of like isolates, representatives were chosen for further study. The gene encoding the $16 \mathrm{~S}$ ribosomal RNA (16S rRNA) was sequenced for all representative isolates, followed by phylogenetic analyses to determine their taxonomic identities. 


\subsection{MATERIALS AND METHODS}

\subsubsection{Bacterial strains and DNA extraction}

The rhizobia used in this study included 76 isolates from the root nodules of 10 Lebeckia plant species (Chapter 2 of this dissertation; note three L. pungens isolates were not analysed). For the SPPCR, rhizobial type strain cultures were also included (Table 3.1). For DNA extraction, isolates were inoculated into Tryptone Yeast (TY) broth as it reduced the slime or gum formation by cultures as compared to growth in Yeast Mannitol (YM) broth. For this purpose, two loop-fulls of a fresh Yeast Mannitol Congo red (YM-CR) agar (Somasagaren \& Hoben, 1994) culture were inoculated into $0.5 \%$ tryptone yeast (TY) (Somasagaren \& Hoben, 1994) broth in an Erlenmeyer flask. The cultures were incubated on an orbital or a reciprocal shaker at $28^{\circ} \mathrm{C}$ until there was sufficient growth (3-5d, approximately mid-log growth phase).

Whole cell DNA was extracted from the 76 rhizobial isolates and type strains, using the protocol described by Wilson (1989). The bacteria in $20 \mathrm{ml}$ broth culture were harvested by centrifugation at $112 \mathrm{x} g$ and $5^{\circ} \mathrm{C}$ for $10-15 \mathrm{~min}$. The pelleted cells were then washed by suspension in $10 \mathrm{mM}$ TrisHCL $(\mathrm{pH} \mathrm{8)}$ wash buffer and centrifuged as before. The washed pellet was then suspended in $2 \mathrm{ml}$ CTAB (N-cetyl-N, N, N-trimethyl ammonium bromide) extraction buffer (CTAB, 5\% w/v; Tris$\mathrm{HCl}, 150 \mathrm{mM}$; EDTA, $40 \mathrm{mM} ; \mathrm{NaCl}, 2 \mathrm{M} ; \mathrm{pH}$ ) and incubated overnight at $4^{\circ} \mathrm{C}$ in the presence of $100 \mu$ l, freshly prepared $0.5 \%$ lysozyme solution (Roche Molecular Biochemicals, Switzerland). To the lysing solution, $100 \mu \mathrm{l} 5 \mathrm{M} \mathrm{NaCl}$ and $200 \mu \mathrm{l} 10 \%$ sodium dodecyl sulphate (w/v) were added and incubated for $20 \mathrm{~min}$ at $65-80^{\circ} \mathrm{C}$. This was followed by the addition of $80 \mu \mathrm{l} \mathrm{CTAB}$ extraction buffer and mixing by inverting three times every $5 \mathrm{~min}$, followed by centrifugation for $5 \mathrm{~min}$ at $10000 \mathrm{x} \mathrm{g}$ at $25^{\circ} \mathrm{C}$. The DNA in the aqueous phase was then precipitated by the addition of 0.6 volumes isopropanol. The precipitated DNA was pelleted by centrifugation at $10000 \mathrm{x} g$, washed with $70 \%$ ethanol, air-dried and dissolved in $50 \mu \mathrm{TE}$ buffer (Tris-HCl, 10mM; 1mM, EDTA; pH 8). Isolates for which this extraction method failed to yield DNA were treated with the Promega Wizard Genomic DNA Purification Kit (Promega, Madison, USA) according to the manufacturer's instructions. The concentration of the extracted DNAs was determined using a NanoDrop spectrophotometer (NanoDrop Technologies, USA). The purified DNA was stored at $-20^{\circ} \mathrm{C}$ until use. 


\subsubsection{Specific primer PCR fingerprinting procedures}

The nif gene derived primer RPO1 (Richardson et al., 1995) and arbitrary sequence primer CRL7 (Mathis and McMillin, 1996) (Table 3.2) were used to fingerprint the 76 Lebeckia rhizobia and the type strains (Tables 3.4). All PCR amplifications were conducted in a total reaction volume of $10 \mu \mathrm{l}$ containing $5 \mathrm{ng} / \mu \mathrm{l}$ DNA, $1.5 \mathrm{mM} \mathrm{MgCl}_{2}, 800 \mu \mathrm{M}$ dNTPs, $1 \mu \mathrm{M}$ primer, and $0.025 \mathrm{U} / \mu \mathrm{l}$ Taq polymerase and its reaction buffers (Promega, Madison, USA). The RPO1 PCR cycling conditions included an initial denaturation at $94^{\circ} \mathrm{C}$ for 3 min followed by 5 cycles of denaturation at $94^{\circ} \mathrm{C}$ for $50 \mathrm{sec}$, annealing at $55^{\circ} \mathrm{C}$ for $1 \mathrm{sec}$ and extension at $72^{\circ} \mathrm{C}$ for $1 \mathrm{sec}$, followed by another 25 cycles each consisting of denaturation at $94^{\circ} \mathrm{C}$ for $45 \mathrm{sec}$, annealing at $60^{\circ} \mathrm{C}$ for $1 \mathrm{sec}$ and extension at $72^{\circ} \mathrm{C}$ for $1 \mathrm{sec}$. The thermal cycling profile for primer CRL7 consisted of 35 cycles of denaturation at $94^{\circ} \mathrm{C}$ for $50 \mathrm{sec}$, annealing at $35^{\circ} \mathrm{C}$ for 2 min and extension at $72^{\circ} \mathrm{C}$ for $2 \mathrm{~min}$, followed by a final extension at $72^{\circ} \mathrm{C}$ for 7 min. PCRs were carried out in a Gene Amp-PCR system 2700 (Applied Biosystems, California USA). Amplified DNA was separated by agarose gel 2.0\% (w/v) electrophoresis in the presence of TAE buffer (Tris-HCl, $40 \mathrm{mM}$; NaOAc, $20 \mathrm{mM}$; EDTA, $1 \mathrm{mM}$; pH8.5) at $667 \mathrm{~V} / \mathrm{cm}$ (Sambrook \& Russell, 2001). The resulting DNA fingerprints were stained in the gels using ethidium bromide (Botha et al., 2004), visualized using ultraviolet light and recorded using a CCD camera. Fingerprints were scored for the presence and absence of bands and subjected to cluster analysis using the combined data for RPO1 and CRL7. The resulting data were analysed using Unweighted Pair Group Method Arithmetic Mean (UPGMA) (Sokal \& Michener, 1958) under the Pearson correlation and Dice band based algorithms (Bionumerics Version 4.0 software, Applied-Maths, St. Martin-Latem, Belgium).

\subsubsection{S rRNA gene sequencing and phylogenetic analyses}

Thirty nine isolates (Table 3.3) were selected as representatives for the various SP-PCR clusters. For these isolates, a portion of the 16S rRNA gene was amplified using the primers 16f 27 and 16r 1485 (Table 3.2; Lane, 1991). PCR amplification was carried out in $50 \mu$ reaction volumes using the same reaction components and concentrations as described for the SP-PCR, except that $0.5 \mu \mathrm{M}$ of each primer was used. PCR was carried out in an Eppendorf Mastercycler Gradient apparatus (Merck chemicals, Johannesburg, SA) using the following thermal profiles: an initial denaturation at $94^{\circ} \mathrm{C}$ for $4 \mathrm{~min}$ followed by 30 cycles of denaturation at $94^{\circ} \mathrm{C}$ for $30 \mathrm{sec}$, annealing at $50^{\circ} \mathrm{C}$ for 30 
sec and extension at $72^{\circ} \mathrm{C}$ for $1 \mathrm{~min}$, followed by a final extension at $72^{\circ} \mathrm{C}$ for $5 \mathrm{~min}$ (Laguerre $e t$ al., 1994). The PCR products were separated by electrophoresis in $1 \%(\mathrm{w} / \mathrm{v})$ agarose gels, TAE buffer and stained as before. The resulting PCR products were purified by precipitation with polyethylene glycol (PEG) (Steenkamp et al., 2006) and sequenced in both directions using the original PCR primers and the ABI PRISM BigDye Terminator v3.0 Cycle Sequencing Kit (Applied Biosystems, Foster City, Calif.) and the 3730 DNA Analyzer (Applied Biosystems).

All raw sequence files were inspected and corrected, where necessary, using Chromas Lite 2.0 (Technelysium) and BioEdit version 5.0.9 (Hall, 1999). All 16S rRNA sequences were also compared to those in GenBank (National Centre for Biotechnology Information; http://www.ncbi.nlm.nih.gov/) using blastn (Altschul et al., 1997. The 16S rDNA sequences for the relevant rhizobial type strains or reference strains were also obtained from GenBank and included together with the Lebeckia rhizobial sequences in multiple alignments. These alignments were constructed using MAFFT version 5.85 (http://align.bmr.kyushu-u.ac.jp/mafft/online/server/) with the L-INS-i option effective (Katoh et al., 2002). To determine the appropriate evolutionary models for phylogenetic analyses, Modeltest 3.7 (Posada \& Crandall, 1998) and PAUP* version 10b (Swofford, 2002) were used. Phylogenies were inferred using maximum likelihood (ML) and neighbor joining (NJ) distance analyses. Bootstrap analyses were based on 1000 replicates and the same best-fit parameters used to infer the individual phylogenies. 


\subsection{RESULTS}

\subsubsection{SP-PCR fingerprint analysis}

Cluster analysis of the SP-PCR fingerprints generated with primers RPO1 and CRL7 separated the 76 Lebeckia rhizobia into ten major clusters or lineages $(\mathrm{C} 1-\mathrm{C} 10)$ at the $\geq 60 \%$ similarity level (Fig. 3.1). Cluster C1 contained the fast-growing Sinorhizobium meliloti (LMG6133) and intermediategrowing Mesorhizobium plurifarium (LMG11892) type strains, the intermediate-growing $L$. cytosoides strains WC19.1b and WC19.1c from Citrusdal and all the intermediate-growing $L$. sericea strains from Bitterfontein and the Kamiesberg, as well as a single fast-growing isolate from L. ambigua at Modder River. The Cluster C1 isolates shared $\geq 65 \%$ similarity. Cluster C2 was made up of two fast-growing Clanwilliam isolates from L. meyerianna and the slow-growing B. japonicum type strain LMG6138, which clustered at $\geq 65 \%$ similarity. The RPO1 profile of LMG6138 appeared different, however, to that of the apparently related fast-growing strains in cluster C2 suggesting its presence in this cluster was an experimental artifact (Fig. 3.1). Cluster C3 was composed of strains sharing $\geq 60 \%$ similarity that were isolated from quite widely separated localities and various species including the fast-growing isolates of L. ambigua (Modder River), the slow-growing L. sepiaria isolate WC121a (Citrusdal), the intermediate-growing L. multiflora isolate WC23.1a (Lambert's Bay) and five fast-growing isolates from L. meyerianna (Clanwilliam). Cluster C4 included two intermediate-growing Lambert's Bay isolates from L. multiflora (WC23.1a and b) that grouped at $72 \%$ similarity with the fast-growing $R$. tropici type strain LMG9503. Intermediate-growing isolate WC23.1d (L. multiflora) formed a single cluster C5 that joined cluster $\mathrm{C} 4$ at $\geq 60 \%$ similarity. Clusters C4 and C5 were linked to C3 at a similarity of about 55\% (Fig. 3.1). Cluster C6 contained the two very slow-growing isolates from $L$. simsiana obtained from Stellenbosch, which grouped at 70\% similarity with the slow-growing B. elkanii type strain LMG6134. Cluster C7 contained all the fast-growing isolates from $L$. spinescens collected at Beaufort West, which grouped together at a high similarity ( $\geq 76 \%)$, and were related to the fast-growing $R$. etli and $R$. leguminosarum type strains, LMG6134 and LMG8820, respectively. Cluster C7 was separated from cluster C9 by a single slow-growing strain (Betal106f) isolated from L. pungens (Meiringspoort), which grouped with low similarity (52\%) to the L. spinescens cluster C7 strains. Cluster C9 contained fast-growing isolates from L. pauciflora collected in the Cedarberg, which shared $\geq 70 \%$ similarity. Cluster C10, at similarities $\geq 75 \%$, included the remaining six very slow-growing strains from L. pungens isolated 
from Meiringspoort, as well as the two slow-growing Cedarberg L. pauciflora isolates WC21.11 and WC21.k, which had identical profiles, and an intermediate-growing L. sericea isolate WC33b1 from Bitterfontein. The presence of intermediate WC33b1 amongst fast growers in C10 may have been an artifact from the CRL7 fingerprint of this strain (Fig 3.1), as the RPO1 profile of this isolate was virtually identical to that of WC33a amongst the other intermediate $L$. sericea isolates in cluster C1 (Fig. 3.1). From the ten clusters, 39 isolates were selected for further taxonomic analysis (Fig. 3.1).

\subsubsection{S rRNA gene sequencing and phylogenetic analysis}

Nearly full length (1200-1500 base pair [bp]) nucleotide sequences were obtained for the 16S rRNA gene from each of the 39 isolates associated with the 10 Lebeckia species. Comparison of these sequences to those in GenBank, using blastn, indicated that 30 represented members of the bacterial division $\alpha$-Proteobacteria, while nine isolates had homology to Burkholderia, a genus in the division $\beta$-Proteobacteria (Table 3.3). Amongst the $\alpha$-Proteobacteria, all of the fast-growing isolates from L. spinescens showed similar identity to Sinorhizobium (which is fast-growing). Thirteen out of the fourteen intermediate-growth rate isolates from L. cytisoides, L. multiflora and L. sericea had $16 \mathrm{~S}$ rRNA sequences similar to those of Mesorhizobium species, an exception being L. sericea strain NK22 which was assigned to Bradyrhizobium (Table 3.3). On the other hand, the slow-growing $L$. sepiaria strain WC12.1a was assigned to Mesorhizobium, whereas the other slow-growing isolates had 16S RNA sequences similar to Bradyrhizobium species including all three from L. pungens, one of the four L. pauciflora isolates and the L. simsiana isolate examined (Table 3.3). Among the $\beta$ Proteobacteria, three isolates from L. ambigua, the remaining three from L. pauciflora and the three L. meyerianna isolates all represented Burkholderia species (Table 3.3).

To determine the possible taxonomic identities for the representative rhizobia included in this study, phylogenetic analyses were performed. Two separate aligned datasets were constructed for the $\alpha$ and $\beta$-Proteobacteria, which included the 16S rRNA sequences for the Lebeckia rhizobia as well as those for the relevant type strains obtained from GenBank. Maximum likelihood (ML) and neighbour joining $(\mathrm{NJ})$ analyses of the $\alpha$-proteobacterial rhizobia generated trees with similar topologies (Fig. 3.2). The isolates prefixed WC12.1a (L. sepiaria), WC19.1b, c (L. cytisoides), WC23.1b, c, d (L. multiflora), WC28.1b, c, e, g, h, i (L. sericea), WC33b, c, e, h (L. sericea), were clearly related to the different Mesorhizobium type strains. The Lebeckia isolates on the $\alpha$ - 
Proteobacteria phylogenetic tree that grouped with Mesorhizobium formed two clusters. One cluster, consisting of four of the seven strains isolated from L. sericea, formed a sister taxon to the type species $M$. loti, $M$. ciceri and $M$. tianshanense, while being more distant to the species $M$. mediterraneum and M. temperatum (Fig. 3.2). In contrast, the other cluster contained 10 isolates that were quite distinct from the mesorhizobial type strains shown in Fig. 3.2. The isolates originating from L. spinescens at Beaufort West, with prefixes $\mathrm{BH}$ or $\mathrm{BW}$, formed a clade very closely related to the Sinorhizobium type strains, with an apparent closer relationship to $S$. arboris, $S$. medicae and $S$. meliloti (Fig 3.2). Five slow-growing isolates and an intermediate isolate NK22, represented Bradyrhizobium species. Of these, four isolates grouped with Br. elkanii including L. pauciflora WC21.11, (Cedarberg) and L. pungens isolates Betal106a, Betal106d, Betal106f (Meiringspoort). The single $L$. simsiana isolate XHR1a was most closely related to Br. betae, with the L. sericea isolate NK22 at its base (Table 3.2).

Within the $\beta$-proteobacterial phylogeny, two clades of Lebeckia rhizobia were identified (Fig. 3.3). Seven Lebeckia rhizobia formed a clade that clustered with the Burkholderia tuberum strain STM 678 originally isolated from the root nodules of Aspalathus carnosa. The two remaining isolates (WC5.4e and WC5.4d) from L. ambigua had identical 16S rRNA sequences and formed a separate cluster with $\mathrm{Bu}$. fungoram strain LMG16225 (Fig. 3.3). The Bu. tuberum group comprised isolates with somewhat dispersed relatedness, including Clanwilliam isolates WC26.1d, WC26.1e and WC26.1f from L. meyeriana and Cedarberg isolate WC21.1j from L. pauciflora. They grouped apart from the other two L. pauciflora isolates WC21.1b and WC21.1i, and both sets were quite distant from the isolate WC5.4c from Modder River, which appeared closely related to L. ambigua (Fig. 3.3). 


\subsection{DISCUSSION}

The taxonomic relationship between the symbiotic rhizobia associated with the indigenous genus Lebeckia species was studied using strains isolated from the root nodules of 10 Lebeckia species. The host plants sampled included species from both the shrubby trifoliate-leaf and suffrutescent needle-leaf types into which this genus is divided (Boatwright et al., 2007; Chapters 1 and 2 of this dissertation). The strains were initially differentiated using SP-PCR fingerprinting, from which 39 representative isolates were selected for further study. Phylogenetic analyses using 16S rRNA sequence information revealed that the Lebeckia species is nodulated by diverse rhizobia representing members of the $\alpha$ - and $\beta$-Proteobacteria. This is the first report concerning the taxonomic identities of the rhizobia associated with the root nodules of this unique legume genus, indigenous to South Africa.

In previous studies, SP-PCR was primarily used to differentiate related strains within a defined species e.g. Bradyrhizobium lupini (Botha et al., 2002) and cowpea-nodulating bradyrhizobia (Law et al., 2007). In the present study, it provided a convenient means to screen the variety of isolates obtained from different Lebeckia species and to select representatives for further taxonomic identification. The results of this fingerprinting analysis (Fig. 3.1) supported previous observations

of the different cultural and nodulation characteristics of these isolates (Chapter 2 of this dissertation) and suggested that many isolates had dissimilar taxonomic identities. Clusters revealed by the combined RPO1 and CRL7 fingerprints generally had excellent correlation with the host plants from which the strains were isolated, notably clusters C1 (L sericea), C7 (L spinescens) and C9 (L pauciflora) and also strain growth rates and collection sites. Within the major clusters, including those containing isolates from several hosts, host-specific sub-clusters could be differentiated at more stringent $(\geq 75 \%)$ similarity values. For example, L. pungens, L. sericea and $L$. pauciflora subgroups could be clearly differentiated within cluster C10 (Fig 3.1) and L. meyerianna and L. ambigua subgroups were also distinct in cluster C3. In ecological studies, previous authors observed that isolates have high sampling site endemicity (Vinuesa et al., 2005a; Law et al., 2007). However, in the present study, most isolates were from individual species sampled at a single collection site, thus suggesting that SP-PCR differentiation among the isolates was probably more species-based (Fig. 3.1). However, there was evidence of locality endemicity in cluster C1, in which 
L. sericea isolates grouped according to their origin at the two sites WC28 and WC33 within the Bitterfontein locality from which they were sampled (Fig. 3.1).

Among the rhizobia selected for 16S rRNA-based studies, each of the ten SP-PCR clusters were represented. In several instances, they included a range of isolates from sub-groups belonging to a particular host species, such as the nine L. spinescens isolates selected from different sub-groups in cluster C7 (Fig. 3.1). Initial comparisons of the 16S rRNA gene sequences to those in GenBank revealed that the selected Lebeckia isolates probably represented both $\alpha$ - and $\beta$-proteobacterial species (Table 3.3). The latter observation was unusual as $\beta$-proteobacterial rhizobia were only recently identified (Moulin et al., 2001; Chen et al., 2003) and seem to be more prominent among the mimosoid legumes (Elliott et al., 2007; Sprent, 2007). The generic relationships revealed by the blastn analyses did, however, match observations of the culture properties of the Lebeckia isolates discussed in Chapter 2 of this dissertation. Based on their 16S rRNA sequences, most of the fastgrowing strains were identified as Sinorhizobium, the intermediate-growing strains as Mesorhizobium and the slow-growers as Bradyrhizobium (Table 3.3, Fig 3.2). Similarly, several others in the fast to intermediate category were found to represent Burkholderia species in the $\beta$ Proteobacteria (Table 3.3, Fig. 3.3). The colonies for the Burkholderia isolates also appeared white and wrinkled (Chapter 2 of this dissertation) as previously reported for bacteria in this genus (Francis et al., 2004; Howard and Inglis, 2003).

Based on 16S rRNA sequence analysis (Fig. 3.2), the Lebeckia rhizobia included in the Mesorhizobium and Sinorhizobium clades probably represent novel, as yet undescribed, species. Four Mesorhizobium strains clustered together in a clade that appeared related to M. tianshanense, M. loti and M. ciceri type strains (Jarvis et al., 1982; Jarvis et al., 1997), although M. loti nodulates species such as Leucaena, Lupinus and Anthyllis that do not occur naturally in South Africa (Young, 1996) and M. ciceri specifically nodulates chickpea (Nour et al., 1994). In contrast, the other Lebeckia mesorhizobia formed a second clade separate from all the Mesorhizobium type species, suggesting that this clade may contain novel species of this genus (Fig. 3.2). It is difficult to say if the two Lebeckia clades might be host-related. The four M. ciceri-M. loti related isolates (WC28.1g, WC33b, WC33c, WC33h) were all from L. sericea in the shrubby Calobota section of Lebeckia (Chapter 2 of this dissertation), but mesorhizobia in the second quite tightly knit clade included 
isolates (WC28.1f, WC19.1b, WC12.1a, WC23.1d, WC33e) from three species in section Calobota (L. sericea, L. cytisoides and L. multiflora) as well as one strain from the suffrutescent section Lebeckia (L. sepiaria isolate WC12.1a).

Within the Sinorhizobium clade, the bacteria isolated from the root nodules of L. spinescens were closely related to one another and did not appear to be near relatives of any other species in this genus (Fig. 3.2). It is interesting to note, however, that the Lebeckia sinorhizobia were most closely associated with a clade that included $S$. arboris, and $S$. medicae and $S$. meliloti. $S$. arboris is an African fast-growing species isolated from the leguminous trees, Acacia senegal and Prosopis chilensis found in Sub-Saharan Africa (Sudan and Kenya) (Nick et al, 1999; Wolde-Meskel et al., 2005), whereas S. medicae and S. meliloti were isolated from Medicago spp. of eastern Mediterranean origin (Rome et al., 1996).

For classification of species in the genus Bradyrhizobium, 16S rRNA gene sequences are of little value as this region is too conserved for differentiation (Vinuesa et al., 2005a, b). The results of this study (Fig. 3.2), did however, indicate that two of the Lebeckia rhizobia from L. sericea and $L$. simsiana, respectively, grouped with $B r$. betae, a species that does not nodulate legumes having been isolated from Beta vulgaris root tumours (Rivas et al., 2004). The four remaining Bradyrhizobium isolates from the shrubby L. pungens (Meiringspoort) and the suffrutescent species L. pauciflora (Cedarberg) appeared to be closely related to Br. elkanii (Fig. 3.2; Table 3.3). Br. elkanii was originally isolated from Glycine max. Determining whether these isolates respectively represent $\mathrm{Br}$. betae and $\mathrm{Br}$. elkanii, will require the analyses of multiple additional housekeeping loci as demonstrated by Vinuesa et al. (2005a, b).

The Lebeckia strains identified as Burkholderia were isolated only from the nodules of suffrutescent needle-leaf species within section Lebeckia. Among these, two 16S rRNA-based clades were evident (Fig. 3.3). In one clade, two evidently identical strains isolated from L. ambigua (Modder river) were closely related to Bu. fungorum, forming a distinct cluster (Fig. 3.3). Bu. fungorum has previously been isolated from the environment, the white-rot fungus Phanerochaete chrysosporium and animal and human tissue samples (Coenye et al., 2001). This species has not been reported to nodulate legumes and its nitrogen-fixing ability has not been studied. The second Lebeckia- 
Burkholderia clade appeared to be quite heterogeneous, with the third isolate from L. ambigua grouping very closely with Bu. tuberum (Fig. 3.3). This Burkholderia species was originally isolated from Aspalathus carnosa in the Western Cape (Moulin, et al., 2001) and has also been shown to nodulate various Cyclopia species and siratro (Elliot et al., 2007; Moulin, et al., 2001; Chen et al., 2003). Further investigation is required, however, to confirm whether this Lebeckia strain (WC5.4c) indeed represents Bu. tuberum. The remaining Burkholderia isolates, originating from L. meyeriana (Clanwilliam) and L. pauciflora (Cedarberg), were in separate clusters from WC5.4c (Fig. 3.3). Although more research is required for complete taxonomic characterization of these Lebeckia rhizobia, the results of this study suggests the likely occurrence of Burkholderia species capable of nodulation and nitrogen fixation in regions where suffrutescent species of Lebeckia are found. Burkholderia species were previously isolated from Aspalathus and Cyclopia spp. growing in the western and southern Cape regions of South Africa (Moulin et al., 2001, Kock, 2004). A number of Burkholderia species have been identified that are capable of nodulating legumes (Moulin et al., 2001; Chen et al., 2003; Perin et al., 2006). The isolation, in the present study, of Burkholderia from suffrutescent needle-leaf Lebeckia spp. thus complements the observation that nodulation of legumes by this genus is fairly widespread and diverse (Sprent, 2007).

Although it is well established that the taxonomy of the bacterial microsymbiont is unrelated to that of the host legume, rhizobia were initially classified according to cross-inoculation specificity (Somasegaren \& Hoben, 1994). Although this system later proved unworkable (Thies et al., 2001), the existence of nodulation specificity must have dictated which rhizobia became associated with which legumes, thus resulting in certain groups of legumes sharing common symbiotic bacteria (Allen \& Allen,1981; Brenner et al., 2005). This is clearly illustrated by the fact that Mimosa species spread over different continents prefer nodulation by specific Burkholderia species (Elliott et al., 2007; Sprent, 2007), while 'genistoid' legumes such as Lupinus species indigenous to Europe and North and South America are nodulated by specific Bradyrhizobium species (Stepkowski et al., 2005). This apparent link between legume endemicity and rhizobial identity also seems to extend to other African genistoid genera (Crisp et al., 2000). For example, certain species of the 'genistoid' tribe Podalyrieae (e.g. Cyclopia species) and Crotalarieae (e.g. Aspalathus carnosa) are nodulated specifically by Burkholderia species (Kock 2005; Sprent, 2007). However, many African 'genistoid' legumes appear to be nodulated by diverse rhizobia, including Crotalarieae species. For example, 
Crotalaria podocarpa and species in the Listia section of the genus Lotononis are specifically nodulated by methylobacteria (Le Roux, 2003; Jaftha et al., 2002; Samba et al., 1999; 2002; Yates et al., 2007), while Aspalathus linearis (the Rooibos tea legume) is nodulated by slow-growing Bradyrhizobium species (Staphorst \& Strijdom, 1975; Deschodt \& Strijdom, 1976; Dagutat, 1995; Dakora, 1998; Muofhe \& Dakora, 1999). The results of the current study similarly showed that the Crotalarieae genus Lebeckia is also nodulated by diverse rhizobia, which may be unique from previously described rhizobia (Figs 3.2, 3.3).

Taking into account the current taxonomic revision of Lebeckia (Le Roux \& van Wyk, 2007; Boatwright \& van Wyk, personal communication), it is of interest to compare the rhizobial groupings identified in this study with the host-specificity groups identified previously in Chapter 2 of this dissertation (Tables 2.2-2.4). The nodules of species in the shrubby trifoliate-leaf sections Calobota and Stiza yielded rhizobia only of the $\alpha$-proteobacterial group (i.e. Bradyrhizobium, Mesorhizobium, and Sinorhizobium species), while the nodules of species in the suffrutescent needle-leaf section Lebeckia, yielded only $\beta$ - proteobacterial rhizobia, except for L. pauciflora from which one $\alpha$-proteobacterial isolate (WC21.11) was obtained. Correspondingly, cross-inoculation across these major plant divisions generally failed (Table 2.4). Examination of more plant species will help to establish whether this is typical of genera in the two divisions of Lebeckia.

My study highlighted that Lebeckia is nodulated by members of the genera Mesorhizobium, Sinorhizobium, Bradyrhizobium and Burkholderia. The four genera were distinct from each other in growth and symbiotic characteristics (Chapter 2 of this dissertation), while SP-PCR revealed that clustering of the isolates was related both to geographical location and host plant species. The comparison of SP-PCR and 16S rRNA sequence data confirmed that SP-PCR allowed appreciable bacterial discrimination thus providing an effective screening technique for grouping taxonomically similar isolates. Phylogenetic analyses using $16 \mathrm{~S}$ rRNA gene sequences enabled elucidation of the generic status of the various bacteria. However, this region is conserved, thus limiting its discriminatory power and restricting taxonomic resolution when comparing closely related strains (Vandamme et al., 1996). Further taxonomic investigation of the isolates is clearly required, including the sequencing of additional marker genes, DNA-DNA hybridization, and biochemical analysis to clarify their taxonomic position. These polyphasic techniques should help to resolve 
species identities of the various Lebeckia isolates examined in this study. This has relevance, considering present evidence that suffrutescent needle-leaf species of Lebeckia differ in their respective rhizobial affinities from species in the shrubby trifoliate-leaf division, which will become a separate genus (Boatwright \& van Wyk, personal communication). 


\subsection{REFERENCES}

Allen, O. N., \& Allen, E. K. 1981. The Leguminosae: A source book of characteristics, uses and nodulation. MacMillan, London.

Bionumerics Version 4.0 software. 2002. Applied-Maths, Martin-Latem, Belgium.

Boatwright J. S., van Wyk, B .E., \& Tilney, P. M. 2007. Systematic studies in the genus Lebeckia and related genera: A revision of Lebeckia section Stiza (Crotalarieae, Fabaceae). S. Afr. J. Bot. 73: 280

Botha, W. J., Bloem, J. F. \& Law, I. J. 2002. Bradyrhizobium sp. (Lupinus) in the winter rainfall region of South Africa. Biol. Fertil. Soils. 36: 335-343.

Botha, W. J., Jaftha, J. B., \& Bloem, J. F. 2004. Effect of soil bradyrhizobia on the success of soybean inoculant strain CB 1809. Microbiol. Res. 159: 219-231.

Bloem, J. F., Botha, W. J., Law, I. J., \& Steyn P. L. 2002. Colony variation in Sinorhizobium meliloti inoculant strain U 45. Microbiol. Res. 157: 283-292.

Brenner, D. J., Krieg, N. R., \& Staley, J. T. 2005. The Alpha-, Beta-, Delta-, and Epsilonproteobacteria. In: Garrity, G. M. (Ed). Bergey's manual of systematic bacteriology, Second Edition. Springer-Verlag, New York, Hedelberg. http://www.cme.msu.edu/Bergeys/

Chen, W. M., Moulin, L., Bontemps, C., Vandamme, G. B., \& Masson, C. B. 2003. Legume symbiotic nitrogen fixation by $\beta$-Proteobacteria is widespread in nature. J. Bacteriol. 185: 72667272.

Coenye, T., Laevens, S., Willems, A., Ohlen, M., Hannant, W., Govan, J. R. W., Gillis, M. Falsen, E., \& Vandamme, P. 2001. Burkholderia fungorum sp. nov. and Burkholderia caledonica sp. nov., two new species isolated from the environment, animals and human clinical samples. Int. J. Syst. Evol. Microbiol. 51: 1099-1107. 
Crisp M. D., Gilmore, S., \& Van Wyk, B. E. 2000. Molecular phylogeny of the genistoid tribes of Papilionoid legumes. In: Herendeen, P. S. \& Bruneau, A (Eds.). Advances in legume systematics, part 9. Royal Botanic Gardens, Kew. Pp 249-276.

Dagutat, H. 1995. Identity and taxonomy of indigenous South African rhizobia. PhD thesis. University of Pretoria, Pretoria, Republic of South Africa.

Dakora, F. D. 1998. Nodulation specificity of Aspalathus linearis subsp. Linearis, a shrub tea legume indigenous to the Western Cape. In: Elmerich, C., Kondorosi, A. \& Newton, W. E. (Eds). Biological nitrogen fixation for the $21^{\text {st }}$ Century. Kluwer, Dordrecht. Pp 671-672.

Deschodt, C. C., \& Strijdom, B. W. 1976. Effective nodulation of Aspalathus linearis bradyrhizobia from other Aspalathus species. Phytophylactica. 8: 103-104.

Elliott, G. N., Chen, W. M., Bontemps, C., Chou, J. H., Young, J. P. W. Sprent, J. I., \& James, E. K. 2007. Nodulation of Cyclopia spp. (Leguminosae, Papilionoideae) by Burkholderia tuberum. Ann. Bot. 100: 1403-1411.

Francis, A., Aiyar, S., Yean-Yean, C., Naing, L., \& Ravichandran, M. 2006. An improved selective and differential medium for the isolation of Burkholderia pseudomallei from clinical specimens. Diag. Microbiol. Infect. Dis. 55: 95-99.

Giller, K. E. 2001. Nitrogen fixation in tropical cropping systems, Second edition. CABI, Wallingford.

Hall, T. A. 1999. Bioedit: A user-friendly biological sequence alignment editor and analysis program for Windows 95/98 (Software). North Carolina, State University.

Howard, K., \& Inglis, T. J. J. 2003. Novel selective medium for isolation of Burkholderia pseudomallei. J. Clinical Microbiol. 41: 3312-3316. 
Jaftha, J. B., Strijdom, B. W., \& Steyn, P.L. 2002. Characterization of pigmented Methylotrophic bacteria which nodulate Lotononis bainesii. Syst. Appl. Microbiol. 25: 440-449.

Jarvis, B. D. W., Pankhurst, C. E., \& Patel, J. J. 1982. Rhizobium loti, a new species of legume root nodule bacteria. Int. J. Syst. Bacteriol. 32: 378-380

Jarvis, B. D. W., van Berkum, P., Chen, W. X., Nour, S. M., Fernandez, M. P., Cleyet-Marel, J. C., \& Gillis, M. 1997. Transfer of Rhizobium loti, Rhizobium haukii, Rhizobium ciceri, Rhizobium medittereneum and Rhizobium tianshanense to Mesorhizobium gen. nov. Int. J. Syst. Bacteriol. 47: 895-898.

Jordan, D. C. 1984. Family III Rhizobiaceae Conn 1938. In: Krieg, N.R. \& Holt, J. G. (Eds) Bergey's manual of systematic bacteriology. William and Wilkins, Baltimore. Pp 234-243.

Katoh, K., Misawa, K., Kuma, K. I., \& Miyata, T. 2002. MAFFT: a novel method for rapid multiple sequence alignment based on fast Fourier transform. Nucl. Acids Res. 30: 3059-3066.

Kock, M. M. 2004. Diversity of root nodulating bacteria associated with Cyclopia species. PhD. thesis. University of Pretoria, Pretoria, Republic of South Africa.

Kuykendall, L. D., Saxena, B., Devine, T. E., \& Udell, S. E. 1992. Genetic diversity in Bradyrhizobium japonicum Jordan 1982 and a proposal for Bradyrhizobium elkanii sp. nov. Can. J. Microbiol. 38: 501-505.

Laguerre, G., Allard, M. R., Revoy, F., \& Amarger, N. 1994. Rapid identification of rhizobia by restriction fragment length polymorphism analysis of PCR-amplified 16S rRNA genes. Appl. Environ. Microbiol. 60: 56-63.

Lane, D. J. 1991. 16S/23S rRNA sequencing. In: E. Stackebrandt and M. Goodfellow (Eds.), Nucleic acid techniques in bacterial systematics. John Wiley and Sons, New York, NY. Pp. 115175. 
Law, I. J., Botha, W. J., Majaule, U. C., \& Phalane, F.L. 2007. Symbiotic and genomic diversity of "Cowpea" bradyrhizobia from soils in Botswana and South Africa. Biol. Fertil. Soils. 43: 653-663.

Le Roux, J. J. 2003. The diversity of root nodule bacteria associated with indigenous Lotononis spp. as determined by sodium dodecyl-sulphate polyacrylamide gel electrophosis and 16S rDNA sequencing. MSc. thesis. University of Pretoria, Pretoria, Republic of South Africa.

Le Roux M. M., \& van Wyk, B. E. 2007. A revision of Lebeckia sect. Lebeckia: The L. sepiaria group. S. Afr. J. Bot. 73: 118-130.

Lindique, M. I. 2005. Diversity of rhizobia nodulating Phaseolus vulgaris and Phaseolus coccineus in South Africa. MSc. thesis. University of Pretoria, Pretoria, Republic of South Africa.

Mathis, J. N., \& McMillin, D. E. 1996. Detection of genetic variation in Bradyrhizobium japonicum USDA 110 variants using DNA fingerprints generated with GC rich arbitrary PCR primers. Plant Soil. 186: 81-85.

Muofhe, M. L., \& Dakora, F. D. 1999. Nitrogen nutrition in nodulated field plants of shrub tea legume Aspalathus linearis assessed using ${ }^{15} \mathrm{~N}$ natural abundance. Plant Soil. 209: 181-186.

Moulin, L., Munive, A., Dreyfus, B., \& Boivin-Masson, C. 2001. Nodulation of legumes by members of the $\beta$-subclass of Proteobacteria. Nature. 411: 948-950.

Nick, G., de Lajudie, P., Eardly, B. D., Suomalainen, S., Paulin, L., Zhang, X., Gillis, M., \& Lindstrom, K. 1999. Sinorhizobium arboris sp. Nov. and Sinorhizobium kostiense sp. nov., isolated from leguminous trees in Sudan and Kenya. Int. J. Syst. Bacteriol. 49: 1359-1369.

Nour, S. M., Fernandez, M. P. Normand, P., \& Cleyet-Merel, J. C. 1994. Rhizobium ciceri sp. nov., consisting of strains that nodulates chickpeas (Cicer arietinum L). Int. J. Syst. Bacteriol. 44: 511522. 
Perin, L., Martinez-Aguilar, L., Castro-Gonzalez, R.,Estrada-de los Santos, P., Cabellos-Avelar,T., Guedes, H. V., Reis, V. M., \& Caballero-Mellado, J. 2006. Diazotrophic Burkholderia species associated with field-grown maize and sugar cane. Appl. Environ. Microbiol. 72: 3103-3110.

Posada, D., \& Crandall, K. A. 1998. MODELTEST: testing the model of DNA substitution. Bioinformatics. 14: 817-818.

Richardson, A. E., Viccars, L. A., Watson, J. M., \& Gibson A. H. 1995. Differentiation of Rhizobium strains using the polymerase chain reaction with random and directed primers. Soil Biol. Biochem. 27: 515-524.

Rivas, R., Willems, A., Palomo, J. L. Garcia-Benavides, P., Mateos, P. F., Martinez-Molina, E., Gillis, M., \& Velázquez, E. 2004. Bradyrhizobium betae sp. nov., isolated from roots of Beta vulgaris affected by tumour-like deformations. Int. J. Syst. Evol. Microbiol. 54: 1271-1275.

Rome, S., Fernandez, M. P., Brunel, B., Normand, P. \& Cleyet-Marel, J. C. 1996. Sinorhizobium medicae sp. nov., isolated from annual Medicago spp. Int. J. Syst. Bacteriol. 46: 972-980.

Samba, R. T., de Lajudie, P., Gillis, M., Neyra, M., Barreto, M. M. S., \& Dreyfus, B. 1999. Diversity of rhizobia nodulating Crotalaria spp. from Senegal. Symbiosis. 27: 259-268.

Samba, R. T., Sylla, S. N., Neyra, M., Gueye, M., Dreyfus, B., \& Ndoye, I.. 2002. Biological nitrogen fixation in Crotalaria species estimated using ${ }^{15} \mathrm{~N}$ isotope dilution method. Afr. $J$. Biotechnol. 1: 17-22.

Sambrook, J., \& Russell, D. W. 2001. Molecular cloning. A laboratory Manual. $3^{\text {rd }}$ Edition. Cold Spring Harbor Laboratory Press, Cold Spring Harbor, New York.

Sokal, R. R., \& Michener, C. D. 1958. A statistical method for evaluating systematic relationships. University of Kansans. Science Bulletin. 
Somasegaran, P., \& Hoben, H. J. 1994. Hand book for Rhizobia. Springer-Verlag, New York.

Sprent, J. I. 2007. Evolving ideas of legume evolution and diversity: a taxonomic perspective on the occurrence of nodulation. New Phytol. 174: 1-25.

Staphorst, J.L., \& Strijdom, B. W. 1975. Specificity in the Rhizobium symbiosis of Aspalathus linearis (Burm. Fil.) R. Dahlgr ssp. linearis. Phytophylactica. 7: 95-96.

Steenkamp, E.T., Wright, J., \& Baldauf, S.L. 2006. The protistan origins of animals and fungi. Mol. Biol. Evol. 23: 93-106.

Stepkowski, T., Moulin, L., Krzyzanska, A., McInnes, A., Law I.J., \& Howieson, J. 2005. European origin of Bradyrhizobium populations infecting Lupins and Serradella in soils of Western Australia and South Africa. Appl. Environ. Microbiol. 71: 7041-7052.

Swofford, D. L. 2002. PAUP* Version 4: Phylogenetic analysis using Parsimony and other methods (Software). Sinauer associates, Sunderland, MA.

Sy, A., Giraud, E., Jourand, P., Garcia, N., Willems, A., de Lajudie, P., Prin, Y., Neyra, M., Gillis, M., Boivin-Masson, C., \& Dreyfus, B. 2001. Methylotrophic Methylobacterium bacteria nodulate and fix nitrogen in symbiosis with legumes. J. Bacteriol. 183: 214-220.

Thies, J. E., Holmes, E. M., \& Vachot, A. 2001. Application of molecular techniques to studies in Rhizobium ecology. Australian J. Exp. Agric. Rev. 41: 299-319.

Vandamme, P., Pot, B., Gillis, M., Devos, P., Kersters, K., \& Swings, J. 1996. Polyphasic taxonomy, a consensus approach to bacterial systematics. Microbiol. Rev. 60: 407-438.

Van Heerden, F. R., van Wyk, B. -E. Viljoen, A. M., \& Steenkamp, P. A. 2003. Phenolic variation in wild populations of Aspalathus linearis (rooibos tea). Biochem. Syst. Ecol. 31: 885-895. 
Vinuesa, P., Silva, C., Werner, D., \& Martinez-Romero, E. 2005a. Population genetics and phylogenetic inference in bacterial molecular systematics: the roles of migration and recombination in Bradyrhizobium species cohesion and delineation. Mol. Phylogen. Evol. 34: 29-54.

Vinuesa, P., Leon-Barrios, M., Silva, C., Willems, A., Jarabo-Lorenzo, A., Perez-Galdona, R., Wener, D., \& Martinez-Romero, E. 2005b. Bradyrhizobium canariense sp. nov., an acid-tolerant endosymbiont that nodulates endemic genistoid legumes (Papilionoideae: Genisteae) from the Canary Islands, along with Bradyrhizobium japonicum bv. Genistearum, Bradyrhizobium genospecies $\alpha$ and Bradyrhizobium genospecies beta. Int. J. Syst. Evol. Microbiol. 55: 569-575.

Wilson, K. 1989. Preparation and analysis of DNA. In: Current Protocols in Molecular Biology, volume 1. Ausubel, F. M., Brent, R., Kingston, R. E., Moore, D. D., Seidman, J. G., Smith, J. A. \& Struhl, K. Wiley, New York.

Wolde-Meskel, E., Terefework, Z., Frostegard, A., \& Lindstrom, K. 2005. Genetic diversity and phylogeny of rhizobia isolated from agroforestry legume species in Southern Ethiopia. Int. J. Syst. Evol. Microbiol. 55: 1439-1452

Yates, R. J., Howieson, J. G., Reeve, W. G., Nandasena, K. G., Law, I. J., Brau, L., Ardley, J. K., Nistelberger, H. M., Real, D., \& O’Hara, G. W. 2007. Lotononis angolensis forms nitrogen fixing, lupinoid nodules with phylogenetically unique, fast-growing, pink-pigmented bacteria, which do not nodulate L. bainesii or L listii. Soil Biol. Biochem. 39: 1680-1688.

Young, J. P .W. 1996. Phylogeny and taxonomy of rhizobia. Plant Soil. 186: 45-52.

Young, J. P. W., \& Haukka, K. E. 1996. Diversity and phylogeny of rhizobia. New Phytol. 133: 87-94 


\subsection{TABLES}

Table 3.1. The rhizobial type strains from the South African Rhizobium Culture Collection used for DNA fingerprinting comparison with DNA of Lebeckia rhizobia.

\begin{tabular}{ll}
\hline Species & Type strain $^{\mathrm{a}}$ \\
\hline$R$. tropici & LMG 9503 \\
$R$. etli & LMG 17827 \\
$R$. leguminosarum & LMG 8820 \\
Br. elkanii & LMG 6134 or USDA 76 \\
Br. japonicum & LMG 6138 or WB86 \\
$M$. plurifarium & LMG 11892 \\
$S$. meliloti & LMG 6133 \\
\hline
\end{tabular}

${ }^{a}$ LMG, Laboratorium voor Microbiologie, Universiteit Gent, Belgium; USDA, U.S. Department of Agriculture, Beltsville, Maryland; 
Table 3.2. Oligonucleotides and primers used in this study.

\begin{tabular}{llll}
\hline Primer & Sequence (5' to 3') & Target gene & Reference \\
\hline RPO1 & aatttcaagcgtcgtgcca & Nif gene and random & Richardson et al., 1995 \\
CRL7 & gccegccgcc & random & Mathis \& MacMillin, 1996 \\
16f 27 & agagtttgatcctggctcag & 16S rRNA & Lane, 1991 \\
16r 1485 & taccttgttacgacttcaccca & 16S rRNA & Lane, 1991 \\
\hline
\end{tabular}


Table 3.3. Accession numbers and strain identities of the top blastn GenBank hits for the 16S rRNA sequences of the Lebeckia rhizobia studied. As on 10 April 2008.

\begin{tabular}{|c|c|c|c|c|c|c|}
\hline Isolate & $\begin{array}{l}\text { blastn Top hit } \\
\text { (Accession } \\
\text { no.) }\end{array}$ & $\begin{array}{l}\text { blastn Top hit } \\
\text { (species identity) }\end{array}$ & $\begin{array}{c}\% \\
\text { similarity }\end{array}$ & $\begin{array}{l}\text { Lebeckia } \\
\text { host }\end{array}$ & Habit & Locality \\
\hline BW1LSab & EF152475.1 & Sinorhizobium sp. STM 4036 & 100 & L. spinescens & Shrubby & Beaufort West \\
\hline BW2LSa & EF152475.1 & Sinorhizobium sp. STM 4036 & 100 & L. spinescens & Shrubby & Beaufort West \\
\hline BW2LSb & EF152475.1 & Sinorhizobium sp. STM 4036 & 100 & L. spinescens & Shrubby & Beaufort West \\
\hline BW3LSc & $\mathrm{EF} 152475.1$ & Sinorhizobium sp. STM 4036 & 100 & L. spinescens & Shrubby & Beaufort West \\
\hline BW3LSd & EF152475.1 & Sinorhizobium sp. STM 4036 & 100 & L. spinescens & Shrubby & Beaufort West \\
\hline BH1LSc & EF152475.1 & Sinorhizobium sp. STM 4036 & 100 & L. spinescens & Shrubby & Beaufort West \\
\hline BH2LSa & EF152475.1 & Sinorhizobium sp. STM 4036 & 100 & L. spinescens & Shrubby & Beaufort West \\
\hline BH2LSc & EF152475.1 & Sinorhizobium sp. STM 4036 & 100 & L. spinescens & Shrubby & Beaufort West \\
\hline BH3LSc & $\mathrm{EF} 152475.1$ & Sinorhizobium sp. STM 4036 & 100 & L. spinescens & Shrubby & Beaufort West \\
\hline Betal106a & EU481825.1 & Bradyrhizobium elkaniiSEMIA938 & 99 & L. pungens & Shrubby & Meiringspoort \\
\hline Betal106d & AY923031.1 & Bradyrhizobium sp.LMTR21 & 99 & L. pungens & Shrubby & Meiringspoort \\
\hline Betal106f & AB367695.1 & Bradyrhizobium sp. KO14 & 100 & L. pungens & Shrubby & Meiringspoort \\
\hline NK22 & EF638789.1 & Bradyrhizobium sp. LAR-20 & 100 & L. sericea & Shrubby & Kamiesberg \\
\hline WC19.1a & EU5145528.1 & Mesorhizobium sp. SCAU13 & 99 & L. cytisoides & Shrubby & Citrusdal \\
\hline WC19.1b & EU514526.1 & Mesorhizobium sp. SCAU9 & 99 & L. cytisoides & Shrubby & Citrusdal \\
\hline WC23.1b & EU5145528.1 & Mesorhizobium sp. SCAU13 & 100 & L. multiflora & Shrubby & Lamberts bay \\
\hline WC23.1c & EU514526.1 & Mesorhizobium sp. SCAU9 & 100 & L. multiflora & Shrubby & Lamberts bay \\
\hline WC23.1d & EU514526.1 & Mesorhizobium sp. SCAU9 & 100 & L. multiflora & Shrubby & Lamberts bay \\
\hline WC28.1f & EU514526.1 & Mesorhizobium sp. SCAU13 & 99 & L. sericea & Shrubby & Bitterfontein \\
\hline WC28.1g & EF611375.1 & Mesorhizobium sp. N46 & 100 & L. sericea & Shrubby & Bitterfontein \\
\hline WC28.1h & EU514526.1 & Mesorhizobium sp. SCAU9 & 100 & L. sericea & Shrubby & Bitterfontein \\
\hline WC28.1i & EU514526.1 & Mesorhizobium sp. SCAU13 & 99 & L. sericea & Shrubby & Bitterfontein \\
\hline WC28.1j & EU514526.1 & Mesorhizobium sp. SCAU9 & 100 & L. sericea & Shrubby & Bitterfontein \\
\hline WC33b & EF611375.1 & Mesorhizobium sp. N46 & 100 & L. sericea & Shrubby & Bitterfontein \\
\hline WC33c & EF611375.1 & Mesorhizobium sp. N46 & 100 & L. sericea & Shrubby & Bitterfontein \\
\hline wC33e & EU514526.1 & Mesorhizobium sp. SCAU9 & 100 & L. sericea & Shrubby & Bitterfontein \\
\hline WC33h & EF611375.1 & Mesorhizobium sp. N46 & 99 & L. sericea & Shrubby & Bitterfontein \\
\hline WC5.4c & AY178072.1 & Burkholderia sp. CI 3 & 100 & L. ambigua & Suffrutescent & Modder R. \\
\hline WC5.4d & AJ971352.1 & Burkholderia sp. C1 & 100 & L. ambigua & Suffrutescent & Modder R. \\
\hline WC5.4e & AJ971352.1 & Burkholderia sp. C1 & 100 & L. ambigua & Suffrutescent & Modder R. \\
\hline WC12.1a & EU514526.1 & Mesorhizobium sp. SCAU9 & 100 & L. sepiaria & Suffrutescent & Citrusdal \\
\hline WC21.1b & AY691395.1 & Burkholderia sp. hpud12.1 & 100 & L. pauciflora & Suffrutescent & Cedarberg \\
\hline WC21.1i & AY691395.1 & Burkholderia sp. hpud12.1 & 100 & L. pauciflora & Suffrutescent & Cedarberg \\
\hline WC21.1j & AY691395.1 & Burkholderia sp. hpud12.1 & 99 & L. pauciflora & Suffrutescent & Cedarberg \\
\hline WC21.11 & EU170551.1 & B. elkanii CCBAU 15609 & 99 & L. pauciflora & Suffrutescent & Cedarberg \\
\hline WC26.1d & AY539823.1 & Burkholderia sp. 59-VN4-1W & 99 & L. meyeriana & Suffrutescent & Clanwilliam \\
\hline WC26.1e & AY539823.1 & Burkholderia sp. 59-VN4-1W & 99 & L. meyeriana & Suffrutescent & Clanwilliam \\
\hline WC26.1f & AY539823.1 & Burkholderia sp. 59-VN4-1W & 99 & L. meyeriana & Suffrutescent & Clanwilliam \\
\hline XHR1a & AF408947.1 & Bradyrhizobium sp. Ellin 105 & 100 & L. simsiana & Suffrutescent & Stellenbosch \\
\hline
\end{tabular}




\subsection{FIGURES}

See following pages. 
RPPO1+CR17

RPO1

CRL7

Isolate
number

Growth
Rate

Host

Host

Geographic

$\%$ similarity

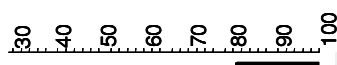

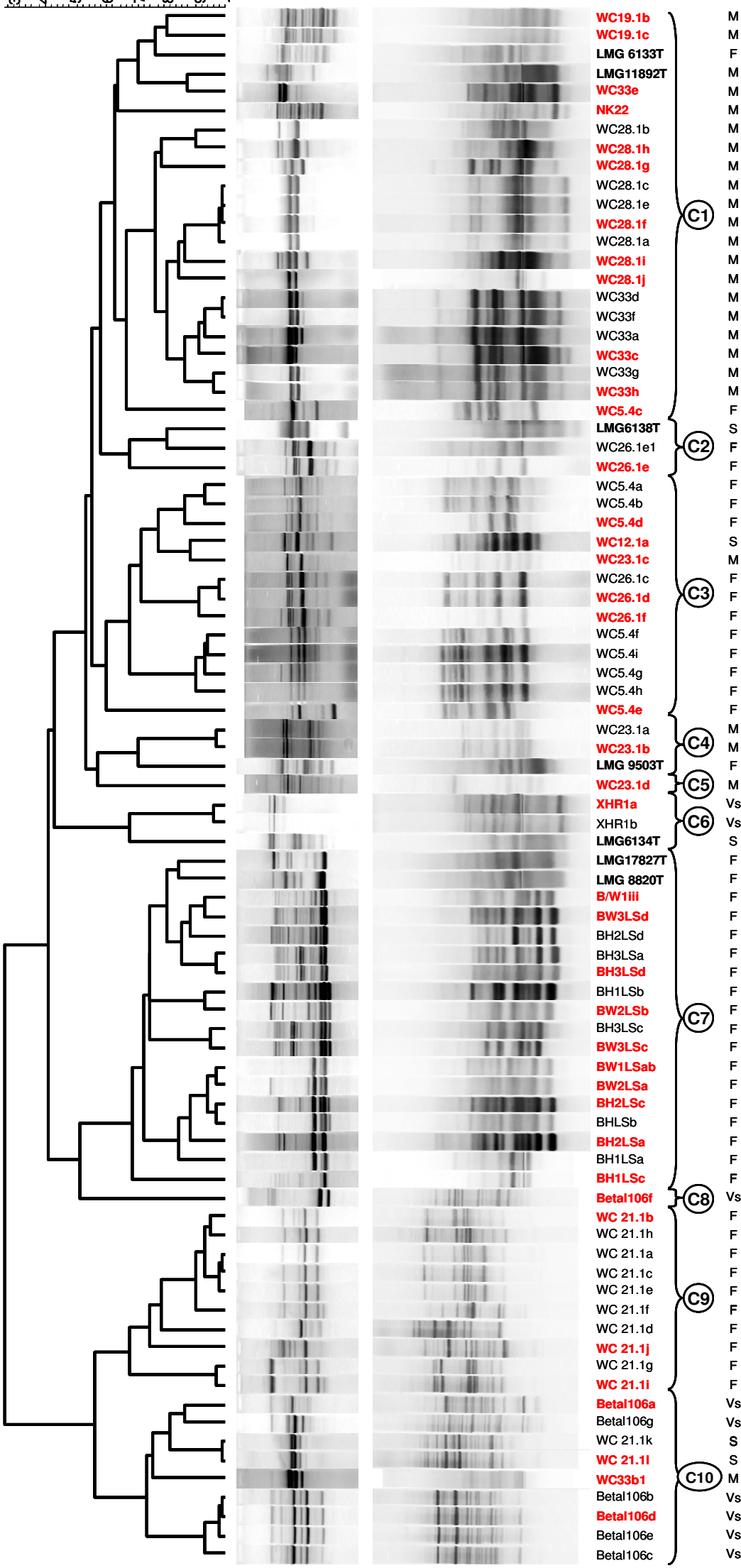

\begin{tabular}{|c|c|c|}
\hline L. cytisoides & Calobota & Citrusdal \\
\hline L. cytisoides & Calobota & Citrusdal \\
\hline Medicago sp. & $\mathrm{N} / \mathrm{a}$ & $\mathrm{N} / \mathrm{a}$ \\
\hline Neptunia sp. & $\mathrm{N} / \mathrm{a}$ & $\mathrm{N} / \mathrm{a}$ \\
\hline L. sericea & Calobota & Bitterfontein \\
\hline L. sericea & Calobota & Kamiesberg \\
\hline L. sericea & Calobota & Bitterfontein \\
\hline L. sericea & Calobota & Bitterfontein \\
\hline L. sericea & Calobota & Bitterfontein \\
\hline L. sericea & Calobota & Bitterfontein \\
\hline L. sericea & Calobota & Bitterfontein \\
\hline L. sericea & Calobota & Bitterfontein \\
\hline L. sericea & Calobota & Bitterfontein \\
\hline L. sericea & Calobota & Bitterfontein \\
\hline L. sericea & Calobota & Bitterfontein \\
\hline L. sericea & Calobota & Bitterfontein \\
\hline L. sericea & Calobota & Bitterfontein \\
\hline L. sericea & Calobota & Bitterfontein \\
\hline L. sericea & Calobota & Bitterfontein \\
\hline L. sericea & Calobota & Bitterfontein \\
\hline L. sericea & Calobota & Bitterfontein \\
\hline L. ambigua & Lebeckia & Modder river \\
\hline Glycine max & $\mathrm{N} / \mathrm{a}$ & $\mathrm{N} / \mathrm{a}$ \\
\hline L. meyerianna & Lebeckia & Clanwilliam \\
\hline L. meyerianna & Lebeckia & Clanwilliam \\
\hline L. ambigua & Lebeckia & Modder river \\
\hline L. ambigua & Lebeckia & Modder river \\
\hline L. ambigua & Lebeckia & Modder river \\
\hline L. sepiaria & Lebeckia & Citrusdal \\
\hline L. multiflora & Calobota & Lamberts bay \\
\hline L. meyerianna & Lebeckia & Clanwilliam \\
\hline L. meyerianna & Lebeckia & Clanwilliam \\
\hline L. meyerianna & Lebeckia & Clanwilliam \\
\hline L. ambigua & Lebeckia & Modder river \\
\hline L. ambigua & Lebeckia & Modder river \\
\hline L. ambigua & Lebeckia & Modder river \\
\hline L. ambigua & Lebeckia & Modder river \\
\hline L. ambigua & Lebeckia & Modder river \\
\hline L. multiflora & Calobota & Lamberts bay \\
\hline L. multiflora & Calobota & Lamberts bay \\
\hline Leucaena sp. & $\mathrm{N} / \mathrm{a}$ & $N / a$ \\
\hline L. multiflora & Calobota & Lamberts bay \\
\hline L. simsiana & Lebeckia & Stellenbosch \\
\hline L. simsiana & Lebeckia & Stellenbosch \\
\hline Glycine max & $\mathrm{N} / \mathrm{a}$ & $\mathrm{N} / \mathrm{a}$ \\
\hline Phaseolus vulgaris & $\mathrm{N} / \mathrm{a}$ & $\mathrm{N} / \mathrm{a}$ \\
\hline Trifolium sp. & $\mathrm{N} / \mathrm{a}$ & $\mathrm{N} / \mathrm{a}$ \\
\hline L. spinescens & Calobota & Beaufort West \\
\hline L. spinescens & Calobota & Beaufort West \\
\hline L. spinescens & Calobota & Beaufort West \\
\hline L. spinescens & Calobota & Beaufort West \\
\hline L. spinescens & Calobota & Beaufort West \\
\hline L. spinescens & Calobota & Beaufort West \\
\hline L. spinescens & Calobota & Beaufort West \\
\hline L. spinescens & Calobota & Beaufort West \\
\hline L. spinescens & Calobota & Beaufort West \\
\hline L. spinescens & Calobota & Beaufort West \\
\hline L. spinescens & Calobota & Beaufort West \\
\hline L. spinescens & Calobota & Beaufort West \\
\hline L. spinescens & Calobota & Beaufort West \\
\hline L. spinescens & Calobota & Beaufort West \\
\hline L. spinescens & Calobota & Beaufort West \\
\hline L. spinescens & Calobota & Beaufort West \\
\hline L. pungens & Stiza & Meiringspoort \\
\hline L. pauciflora & Lebeckia & Cedarberg \\
\hline L. pauciflora & Lebeckia & Cedarberg \\
\hline L. pauciflora & Lebeckia & Cedarberg \\
\hline L. pauciflora & Lebeckia & Cedarberg \\
\hline L. pauciflora & Lebeckia & Cedarberg \\
\hline L. pauciflora & Lebeckia & Cedarberg \\
\hline L. pauciflora & Lebeckia & Cedarberg \\
\hline L. pauciflora & Lebeckia & Cedarberg \\
\hline L. pauciflora & Lebeckia & Cedarberg \\
\hline L. pauciflora & Lebeckia & Cedarberg \\
\hline L. pungens & Stiza & Meiringspoort \\
\hline L. pungens & Stiza & Meiringspoort \\
\hline L. pauciflora & Lebeckia & Cedarberg \\
\hline L. pauciflora & Lebeckia & Cedarberg \\
\hline L. sericea & Calobota & Bitterfontein \\
\hline L. pungens & Stiza & Meiringspoort \\
\hline L. pungens & Stiza & Meiringspoort \\
\hline L. pungens & Stiza & Meiringspoort \\
\hline L. pungens & Stiza & Meiringspoort \\
\hline
\end{tabular}


Figure 3.1. UPGMA dendogram derived from combined RPO1 and CRL7 PCR-fingerprints of Lebeckia isolates and type strains of Bradyrhizobium, Mesorhizobium, Rhizobium and Sinorhizobium species. The type strains are indicated with T. Symbols C1-C10 indicate major clusters and scale shows percentage similarity. The strains selected for sequencing are highlighted in red. The isolate number, growth rate, host species, host sections and geographic origin are indicated next to each DNA profile. LMG6133T represents S. meliloti, LMG11892 represents M. plurifarium, LMG6128 B. japonicum, LMG9503 R. tropici, LMG6134 B. elkani, LMG17827 R. elti and LMG8820 represents $R$. leguminosarium. 


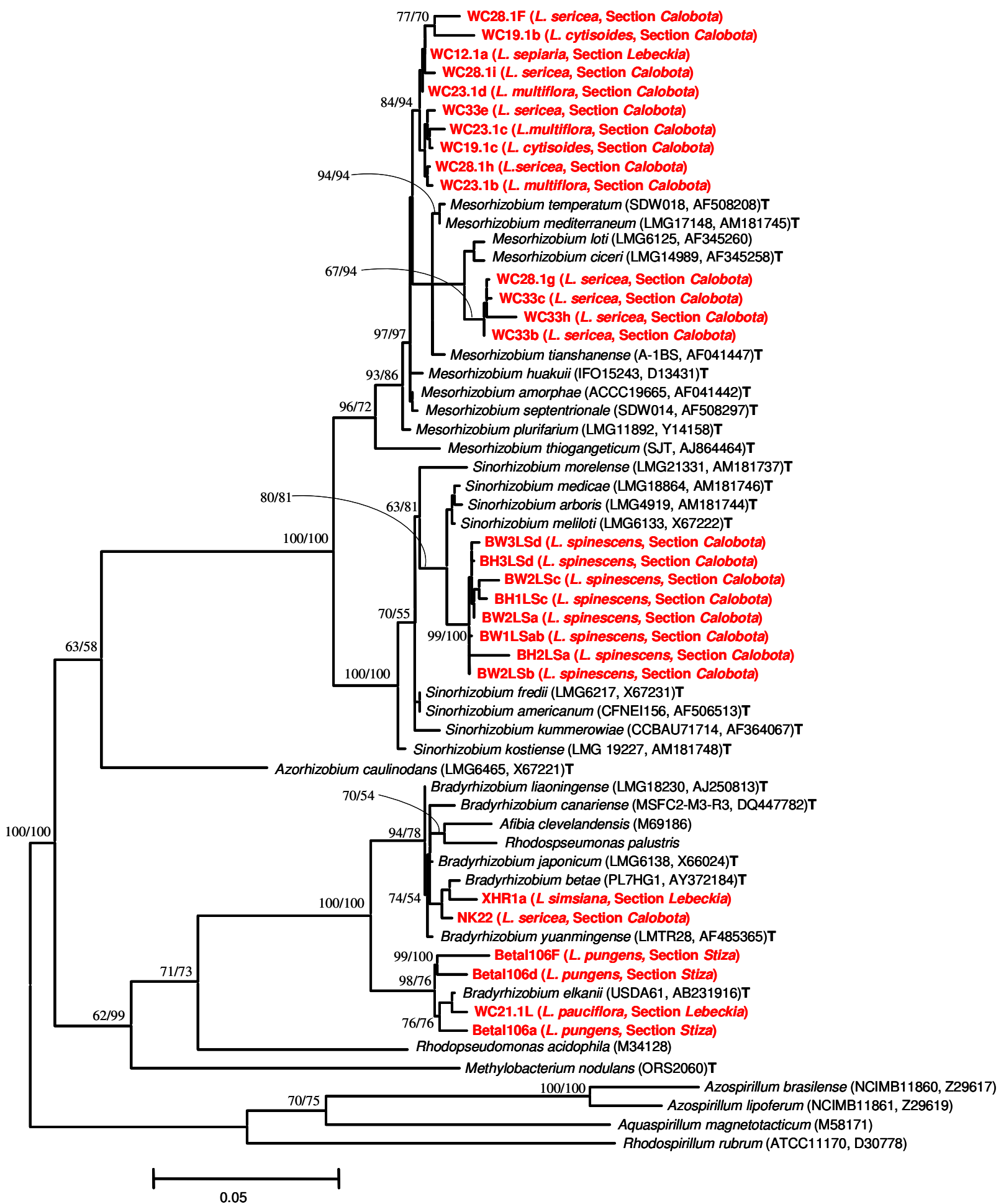

Figure 3.2. A maximum-likelihood (ML) species phylogeny for Lebeckia rhizobia, based on $16 \mathrm{~S}$ rRNA nucleotide sequence data. South African Lebeckia rhizobia are indicated in red and bold. The rhizobia type strains are indicated with a T. The tree was rooted with Azospirillum lipoferum and Azospirillum brasilense. 


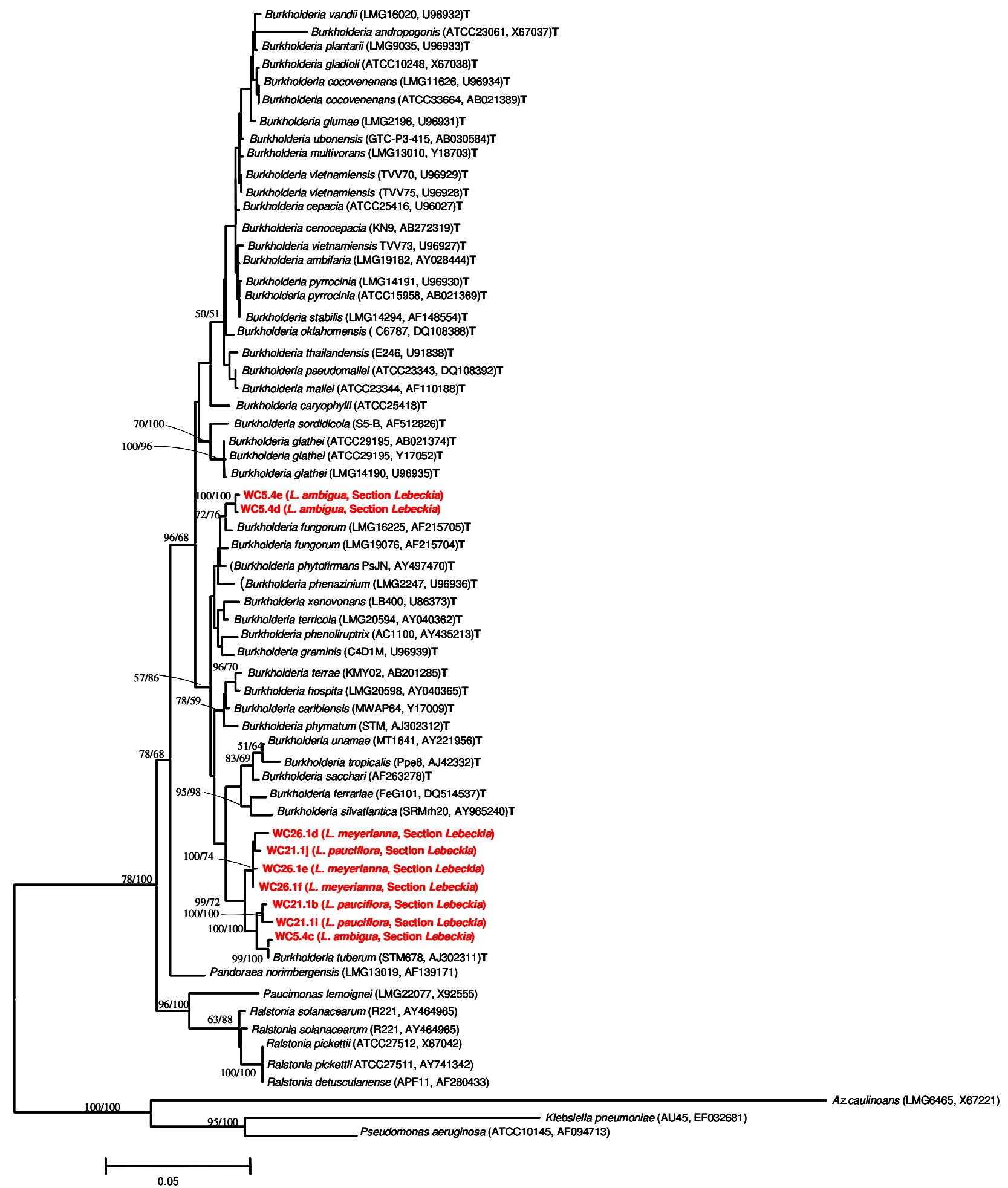

Figure 3.3. A maximum-likelihood (ML) species phylogeny for Burkholderia from Lebeckia, based on 16S rRNA nucleotide sequence data. South African Lebeckia Burkholderia are indicated in red and bold. The Burkholderia type strains are indicated with a T. The tree was rooted with Ralstonia solanacearum and Paucimonas lemoignei. 
CHAPTER 4

CONCLUDING REMARKS

\section{TABLE OF CONTENTS}

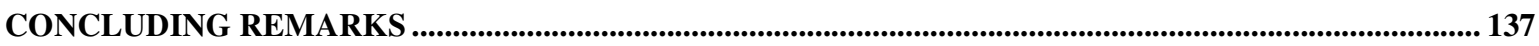

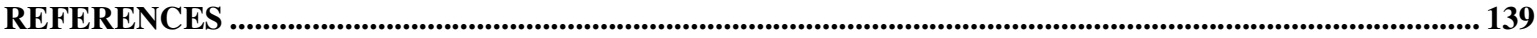

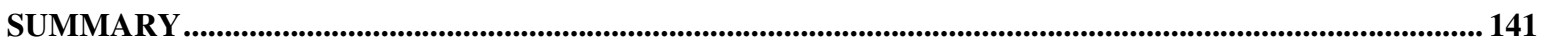




\section{CHAPTER 4}

\section{CONCLUDING REMARKS}

Over the past decade or so there has been a world-wide surge in scientific and commercial interest in native legumes as potential crop plants with novel agricultural or industrial applications (Doyle, 1998; Doyle \& Luckow, 2003; Graham \& Vance, 2003; Sprent, 2007). The additional ecological benefits provided by many of these plants are the result of symbiotic nitrogen fixation by their associated root-nodule bacteria. Considerable effort has consequently been applied to the study of these important and often novel microsymbionts (Moulin et al., 2001; Sy et al., 2001; Rivas et al., 2003; Perin et al., 2006). In South Africa, with its rich heritage of indigenous legumes (Germishuizen \& Meyer, 2003; Elliot et al., 2007; Le Roux \& van Wyk 2007; Boatwright et al., 2007), the rooibos tea and honey bush tea legumes have already shown economic potential. Rooibos tea (Aspalathus linearis) and honey bush tea (Cyclopia) are now regarded as amongst the healthiest natural herbal teas, without additives, preservatives, colourants and caffeine free. Lebeckia spp. also have potential in veld reclamation and as forage legumes and ornamental plants, thus further studies on this legume and its association with symbiotic nitrogen-fixing rhizobia and their respective properties and taxonomic groupings are essential.

As part of an ongoing survey of South African rhizobia (Dagutat, 1995; Le Roux, 2003; Lindique, 2005), isolates from indigenous species of Lebeckia were examined for symbiotic ability using traditional root nodule bacteria technology, screened for genomic relatedness using DNA fingerprinting methods and their putative taxonomic positions determined using 16S rRNA gene sequencing (Laguerre et al., 1994; Richardson et al., 1995; Thies et al., 2001).

The diverse nature of the rhizobia isolated from Lebeckia was clearly illustrated by the different rhizobial genera that were isolated, sometimes from the same plant species. DNA profiles showed that most of the isolates grouped according to host plant species rather than geographical location. The majority of the isolates were found to be classical genera of rhizobia belonging to the $\alpha$-Proteobacteria (Mesorhizobium, Sinorhizobium and Bradyrhizobium) with a few isolates showing affinity to the $\beta$-proteobacterial genus Burkholderia. This species diversity appears to be related in both symbiotic and taxonomic properties to the diversity found amongst the 33-35 
species of Lebeckia, a genus currently undergoing taxonomic revision (Le Roux \& van Wyk, 2007; Boatwright et al., 2007). Lebeckia may be divided into shrubby trifoliate leaf species and suffrutescent needle leaf species. Interestingly, the results of my cross-inoculation studies indicated that rhizobia isolated from one division rarely nodulated legumes of the other division and any nodules formed were ineffective, i.e. cannot fix nitrogen. In contrast, cross-inoculation between symbionts from within either division always resulted in nodulation. Such nodulation was effective when cross-inoculation was between species within the same section of a division, eg. Calobota plants, and ineffective when between different sections of that division, eg. Stiza and Calobota. Furthermore, species in the shrubby division yielded only $\alpha$-Proteobacteria whereas suffrutescent species yielded predominantly $\beta$-Proteobacteria, Burkholderia being isolated only from species in the latter suffrutescent division. These results of consistent with current plant taxonomic studies elsewhere that will soon assign species in the shrubby division to a new genus separate from those in the suffrutescent division that will remain in the genus Lebeckia (Boatwright \& van Wyk, personal communication).

Further investigation of isolates from the 10 Lebeckia species so far examined, well as new isolates from other species may reveal further insights into the taxonomy of the two legume divisions and their rhizobia. In addition, the phylogenetic groupings of rhizobia emerging from the present study suggest that new species of rhizobia specific for Lebeckia may exist. Further study of these using advanced taxonomic techniques such as DNA-DNA hybridization will be required to determine their exact identity. 


\section{REFERENCES}

Boatwright, J. S., van Wyk, B. E., \& Tilney, P. M. 2007. Systematic studies in the genus Lebeckia and related genera: A revision of Lebeckia section Stiza (Crotalarieae, Fabaceae). S. Afr. J. Bot. 73: 280

Dagutat, H. 1995. Identity and taxonomy of indigenous South African rhizobia. PhD thesis. University of Pretoria, Pretoria, Republic of South Africa.

Doyle, J. J. 1998. Phylogenetic perspectives on nodulation: evolving views of plants and symbiotic bacteria. Trends Plant Sci. 3: 473-478.

Doyle, J. J., \& Luckow, M.A. 2003. The rest of the Iceberg, Legume diversity and evolution in a phylogenetic context. Plant Physiol. 131: 900-910.

Elliot, G. N., Chen, W. M., Bontemps, C., Chou, J. H., Young, J. P. W., Sprent, J. I., \& James, E. K. 2007. Nodulation of Cyclopia spp. (Leguminosae, Papilionoideae) by Burkholderia tuberum. $15^{\text {th }}$ International nitrogen fixation conference, 21-26 January 2007. Cape Town, South Africa.

Graham, P. H., \& Vance, C. P. 2003. Legumes: Importance and constraints to greater use. Plant Physiol. 131: 872-877.

Laguerre, G., Allard, M. R., Revoy, F., \& Amarger, N. 1994. Rapid identification of rhizobia by restriction fragment length polymorphism analysis of PCR-amplified 16S rRNA genes. Appl. Environ. Microbiol. 60: 56-63.

Le Roux, J. J. 2003. The diversity of root nodule bacteria associated with indigenous Lotononis spp. as determined by sodium dodecyl-sulphate polyacrylamide gel electrophosis and 16S rDNA sequencing. MSc. thesis. University of Pretoria, Pretoria, Republic of South Africa.

Le Roux M. M., \& van Wyk, B. E. 2007. A revision of Lebeckia sect. Lebeckia: The L. sepiaria group. S. Afr. J. Bot. 73: 118-130. 
Lindique, M. I. 2005. Diversity of rhizobia nodulating Phaseolus vulgaris and Phaseolus coccineus in South Africa. MSc. thesis. University of Pretoria, Pretoria, republic of South Africa.

Moulin, L., Munive, A., Dreyfus, B., \& Boivin-Masson, C. 2001. Nodulation of legumes by members of the $\beta$-subclass of Proteobacteria. Nature. 411: 948-950.

Perin, L., Martinez-Aguilar, L., Castro-Gonzalez, R.,Estrada-de los Santos, P., CabellosAvelar,T., Guedes, H. V., Reis, V.M., \& Caballero-Mellado, J. 2006. Diazotrophic Burkholderia species associated with field-grown maize and sugar cane. Appl. Environ. Microbiol. 72: 31033110 .

Richardson, A. E., Viccars, L. A., Watson, J. M., \& Gibson, A. H. 1995. Differentiation of Rhizobium strains using the polymerase chain reaction with random and directed primers. Soil Biol. Biochem. 27: 515-524.

Rivas, R., Willems, A., Subba-Rao, N. S., Mateos, P. F., Dazzo, F. B., Kroppenstedt, R. M., Martinez-Molina, E., Gillis, M., \& Velázquez, E. 2003. Description of Devosia neptunae sp. nov. that nodulates and fixes nitrogen in symbiosis with Neptunia natans, an aquatic legume from India. Syst. Appl. Microbiol. 26: 47-53.

Sprent, J. I. 2007. Evolving ideas of legume evolution and diversity: a taxonomic perspective on the occurrence of nodulation. New Phytol. 174: 1-25.

Sy, A., Giraud, E., Jourand, P., Garcia, N., Willems, A., de Lajudie, P., Prin, Y., Neyra, M., Gillis, M., Boivin-Masson, C., \& Dreyfus, B. 2001. Methylotrophic Methylobacterium bacteria nodulate and fix nitrogen in symbiosis with legumes. J. Bacteriol. 183: 214-220.

Thies, J. E., Holmes E. M., \& Vachot, A. 2001. Application of molecular techniques to studies in Rhizobium ecology: Review. Austr. J. Exp. Agric. 41: 299-319. 
The genus Lebeckia Thunb. (Family Leguminosae, subfamily Papilionoideae, tribe Crotalarieae) comprises some 33-35 plant species. In this study, the diversity of root-nodule bacteria (rhizobia) associated with Lebeckia species in South Africa was investigated. Lebeckia specimens were collected from nine localities in the western and southern Cape. Seventy nine isolates obtained from the root nodules of ten Lebeckia species were purified. The strains were confirmed as rhizobia by their ability to nodulate their host plants as well as the promiscuous legumes cowpea and siratro. All the isolates formed nodules and effectively fixed nitrogen on their respective Lebeckia hosts, whereas $56 \%$ of the strains were effective on cowpea and $77 \%$ on siratro. The isolates were further characterized using SP-PCR DNA fingerprinting followed by partial $16 \mathrm{~S}$ rRNA gene sequencing of 39 selected isolates to confirm their taxonomic identity. DNA-fingerprinting profiles showed that most of the isolates grouped according to host plant species rather than geographical location. Based on 16S rRNA phylogenetic analysis, the isolates from Lebeckia comprised a wide diversity of nitrogen-fixing bacteria from both the $\alpha$ and $\beta$-Proteobacteria. Amongst the $\alpha$-Proteobacteria fast-growing isolates were all assigned to the genus Sinorhizobium, intermediate-growers were assigned to the genus Mesorhizobium and very slow-growers to the genus Bradyrhizobium. The $\alpha$-proteobacterial rhizobia all nodulated shrubby trifoliate-leaf Lebeckia spp. In contrast, $\beta$-proteobacterial rhizobia related to the genus Burkholderia only nodulated suffrutescent unifoliate needle-leaf Lebeckia spp. Crossinoculation studies suggest that nodulation is restricted to combinations of rhizobia and Lebeckia from either of the two Lebeckia plant types, but not across these groups. This finding has relevance to a current taxonomic revision of Lebeckia being undertaken elsewhere. 
APPENDICES

\section{TABLE OF CONTENTS}

APPENDIX A: MEDIA, BUFFER AND REAGENTS.

143

APPENDIX B: $\beta$-RHIZOBIA 16S RRNA ALIGNMENT .148

APPENDIX C: $\alpha$-RHIZOBIA 16S RRNA ALIGNMENT. 


\section{APPENDIX A: MEDIA, BUFFER AND REAGENTS}

\section{Nitrogen free nutrient solution (Hoagland's solution)}

Hoagland's solution was prepared by dissolving the following ingredients in 20L sterile distilled water (SDW) and adjusted with $\mathrm{KOH}$ to $\mathrm{pH}$ 6.6-6.8.

$\begin{array}{ll}\mathrm{KCl} & 100 \mathrm{~g} \\ \mathrm{Ca}_{3}(\mathrm{PO} 4)_{2} & 100 \mathrm{~g} \\ \mathrm{CaSO}_{4} \cdot 2 \mathrm{H}_{2} \mathrm{O} & 100 \mathrm{~g} \\ \mathrm{MgSO}_{4} \cdot 7 \mathrm{H}_{2} \mathrm{O} & 156 \mathrm{~g} \\ \text { Nafe solution } & 400 \mathrm{ml} \\ \text { Trace element } & 40 \mathrm{ml}\end{array}$

Nafe solution consists of:

$\mathrm{N}_{2}$ EDTA $\quad 15.6 \mathrm{~g}$

$\mathrm{FeSO}_{4} .7 \mathrm{H}_{2} \mathrm{O} \quad 15 \mathrm{~g}$

Dissolve in $600 \mathrm{ml}$ and oxygenated by vigorous shaking until colour was dark brown. The volume was then made up to1500ml.

Trace element solution ingredients:

$\begin{array}{ll}\mathrm{LiCl} & 0.2 \mathrm{~g} \\ \mathrm{CuSO}_{4} \cdot 5 \mathrm{H}_{2} \mathrm{O} & 1 \mathrm{~g} \\ \mathrm{ZnSO}_{4} \cdot 7 \mathrm{H}_{2} \mathrm{O} & 1 \mathrm{~g} \\ \mathrm{TiO}_{2} & 1 \mathrm{~g} \\ \mathrm{H}_{3} \mathrm{BO}_{3} & 1 \mathrm{~g} \\ \mathrm{AL}_{2}(\mathrm{SO} 4)_{3} & 1 \mathrm{~g} \\ \mathrm{SnCl}_{2} \cdot 2 \mathrm{H}_{2} \mathrm{O} & 0.5 \mathrm{~g} \\ \mathrm{MnCl}_{2} \cdot 4 \mathrm{H}_{2} \mathrm{O} & 7 \mathrm{~g} \\ \mathrm{KI} & 0.5 \mathrm{~g} \\ \mathrm{NiSO}_{4} \cdot 7 \mathrm{H}_{2} \mathrm{O} & 1 \mathrm{~g} \\ \mathrm{Co}\left(\mathrm{NO}_{3}\right)_{2} \cdot 6 \mathrm{H}_{2} \mathrm{O} & 1 \mathrm{~g} \\ \mathrm{KBr} & 0.5 \mathrm{~g} \\ \mathrm{Na}_{2} \mathrm{MoO}_{4} \cdot 2 \mathrm{H}_{2} \mathrm{O} & 5.4 \mathrm{~g}\end{array}$

Dissolved in $1800 \mathrm{ml}$ distilled water and volume adjusted to $2000 \mathrm{ml}$. 


\section{Paraffin sand}

$15 \mathrm{~g}$ paraffin wax

Dissolved in 1L benzene

Mixed with $10 \mathrm{~kg}$ sifted sand and sterilized in a drying oven at $160^{\circ} \mathrm{C}$ for $2 \mathrm{hrs}$.

Yeast Mannitol Congo red (YM-CR) (Vincent 1970)

$\begin{array}{ll}\text { Mannitol } & 10 \mathrm{~g} \\ \mathrm{~K}_{2} \mathrm{HPO}_{4} & 0.5 \mathrm{~g} \\ \mathrm{MgSO}_{4} .7 \mathrm{H}_{2} \mathrm{O} & 0.2 \mathrm{~g} \\ \text { Yeast extracts } & 0.4 \mathrm{~g} \\ \mathrm{NaCl} & 0.1 \mathrm{~g} \\ 4 \%(\mathrm{~m} / \mathrm{v}) \text { Congo red } & 10 \mathrm{ml} \\ \text { Agar } & 15 \mathrm{~g}\end{array}$

Yeast Mannitol Agar slants

Per $1000 \mathrm{ml}$ in distilled water:

$\begin{array}{ll}\text { Mannitol } & 10 \mathrm{~g} \\ \mathrm{~K}_{2} \mathrm{HPO}_{4} & 0.5 \mathrm{~g} \\ \mathrm{MgSO}_{4} .7 \mathrm{H}_{2} \mathrm{O} & 0.2 \mathrm{~g} \\ \text { Yeast extract } & 0.4 \mathrm{~g} \\ \mathrm{NaCl} & 0.1 \mathrm{~g} \\ \mathrm{CaCO}_{3} & 1 \mathrm{~g} \\ \text { Agar } & 15 \mathrm{~g}\end{array}$

Water agar

Per $1000 \mathrm{ml}$ in distilled water

Agar $\quad 15 \mathrm{~g}$ 


\section{Yeast Mannitol Broth}

Per $1000 \mathrm{ml}$ of distilled water

Mannitol $\quad 10 \mathrm{~g}$

$\mathrm{K}_{2} \mathrm{HPO}_{4} \quad 0.5 \mathrm{~g}$

$\mathrm{MgSO}_{4} .7 \mathrm{H}_{2} \mathrm{O} \quad 0.2 \mathrm{~g}$

Yeast extract $\quad 0.4 \mathrm{~g}$

$\mathrm{NaCl} \quad 0.1 \mathrm{~g}$

\section{Tryptone}

Per $1000 \mathrm{ml}$ distilled water

Tryptone $\quad 5 \mathrm{~g}$

Yeast extract $\quad 3 g$

Wash buffer

$\mathrm{NaCl}(0.5 \mathrm{M}) \quad 2.422 \mathrm{~g}$

Tris $(100 \mathrm{mM}) \quad 1.169 \mathrm{~g}$

EDTA $(20 \mathrm{mM}) \quad 1.168 \mathrm{~g}$

Dissolved in 200ml SDW, pH 8.0 adjusted with $\mathrm{HCl}$.

\section{Extraction buffer}

Per 500ml SDW, pH 8.0 adjusted with $\mathrm{HCl}$.

$\begin{array}{ll}\text { Tris }(150 \mathrm{mM}) & 9.084 \mathrm{~g} \\ \text { EDTA }(40 \mathrm{mM}) & 5.845 \mathrm{~g} \\ \mathrm{NaCl}(2 \mathrm{M}) & 58.44 \mathrm{~g}\end{array}$

Sodium Chloride-Tris-EDTA (STET) buffer

Per 200ml SDW, pH 8.0 adjusted with $\mathrm{HCl}$.

$\mathrm{NaCl}(0.1 \mathrm{M})$

$1.168 \mathrm{~g}$

Tris $(50 \mathrm{mM})$

$1.211 \mathrm{~g}$

EDTA (10mM)

$0.584 \mathrm{~g}$

Triton X-100 (0.1\%, v/v) $200 \mu \mathrm{l}$ 
$3 \%$ KOH solution

$\mathrm{KOH} 3 \mathrm{~g}$

SDW 100ml

$44 \%(\mathrm{~m} / \mathrm{v}) \mathrm{CaCl}_{2} .2 \mathrm{H} 2 \mathrm{O}$

$\mathrm{CaCl}_{2} .2 \mathrm{H} 2 \mathrm{O} \quad 4.4 \mathrm{~g}$

Distilled water $\quad 10 \mathrm{ml}$

Tris wash buffer

Per 200ml SDW, pH 8.0 adjusted with $\mathrm{HCl}$

M Tris $(100 \mathrm{mM}) \quad 1.169 \mathrm{~g}$

$\mathrm{NaCl}(0.5 \mathrm{M}) \quad 2.422 \mathrm{~g}$

EDTA $(20 \mathrm{mM}) \quad 1.168 \mathrm{~g}$

\section{Extraction buffer}

Per 500ml SDW, pH 8.0 adjusted with $\mathrm{HCl}$

Tris $(150 \mathrm{mM})$

$9.084 \mathrm{~g}$

$\mathrm{NaCl}(2 \mathrm{M})$

$58.44 \mathrm{~g}$

EDTA (40mM)

$5.845 \mathrm{~g}$

10X TBE buffer

Tris $(1 \mathrm{M})$

$24.2 \mathrm{~g}$

EDTA $(10 \mathrm{mM})$

$0.584 \mathrm{~g}$

Borate $(0.83 \mathrm{M})$

$10.27 \mathrm{~g}$

SDW

$200 \mathrm{ml}$

\section{X TBE buffer}

10X TBE buffer stock $\quad 200 \mathrm{ml}$

SDW

$1800 \mathrm{ml}$

\section{Agarose gel}

For $1.5 \%$ and $2 \%$ agarose gels, mix $0.825 \mathrm{~g}$ and $1.1 \mathrm{~g}$ agarose, respectively with $55 \mathrm{ml}$ SDW 
Chloroform: Iso-amylalcohol (24:1)

Chloroform $\quad 240 \mathrm{ml}$

Iso-amylalcohol $\quad 10 \mathrm{ml}$

$5 \mathrm{M} \mathrm{NaCl}$

$\mathrm{NaCl} \quad 58.44 \mathrm{~g}$

Distilled water $\quad 200 \mathrm{ml}$

$20 \%$ Glycerol

Per $1000 \mathrm{ml}$ solution

Concentrated glycerol $\quad 200 \mathrm{ml}$

SDW

$800 \mathrm{ml}$

$2 \mathrm{M} \mathrm{NaOH}$

$\mathrm{NaOH} \quad 2 \mathrm{~g}$

SDW $\quad 100 \mathrm{ml}$

\section{$1.5 \mathrm{M} \mathrm{KCl}$}

$\mathrm{KCl}$

$111.84 \mathrm{~g}$

SDW

$1000 \mathrm{ml}$

$70 \%$ ethanol

Per $1000 \mathrm{ml}$ solution

$\begin{array}{ll}\text { Absolute ethanol } & 700 \mathrm{ml} \\ \text { Distilled water } & 300 \mathrm{ml}\end{array}$

0.25\% Congo red

Congo red $\quad 1 \mathrm{~g}$

Distilled water $\quad 400 \mathrm{ml}$

10\% Sodium dodecyl sulphate (SDS)

Sodium lauryl sulphate $\quad 4 \mathrm{~g}$

SDW $\quad 40 \mathrm{ml}$ 


\section{APPENDIX B: $\beta$-RHIZOBIA 16S rRNA alignment}

B. andropogonis (ATCC23061) T

$B$. fungorum (LMG19076) T

B. caribiensi (MWAP64)T

B. caryophylii (ATCC25418) T

B. cepacia (ATCC25416) T

B. cocovenenas (ATCC33664) T

$B$. cocovenenas (LMG11626) T

$B$. fungorum (LMG16225)

B. gladioli (ATCC10248) T

B. glathei (LMG14190) T

B. glathei (ATCC29195) T

B. glathei (ATCC29195) T

B. glumae (LMG2196) T

$B$. graminis (C4D1M) T

B. hospita (LMG20598) T

B. mallei (ATCC23344) T

B. multivorans (LMG13010) T

$B$. norimbergensis (Y09879) T

B. phenazinium (LMG2247) T

B. phenoliruptrix (AC1100) T

B. phymatum (STM815) T

B. phytofirmans (PsJN) T

B. pickettii (ATCC27511)T

B. pseudomallei (ATCC23343) T

B. pyrrocinia (LMG14191)T

B. pyrrocinia ATCC15958) T

B. sacchari (AF263278) T

B. silvatlantica (SRMrh-20)T

B. solanacearum (ATCC11696) T

B. sordicola (S5-B) T

B. stabilis (LMG14294) T

$B$. terrae (KMY02) T

B. terriccola (LMG20594) T

$B$. thailandensis (E264) T

B. tropicalis (Ppe8) T

B. tuberum (STM678) T

B. unamae (MT1-641) T

$B$. vandii (LMG16020) T

B. vietnamiensis (AMMD) $\mathrm{T}$

$B$. vietnamiensis (TVV75) T

B. vietnamiensis (TVV70)T

B. plantarii (LMG9035) T

$B$. xenovorans (LB400) T

B. cenocepacia (KN9) T

B. ferrariae (FeG101) T

B. ambifaria (LMG19182) T

B. oklahomensis (C6786) T

$B$. ubonensis (GTC-P3-415) T

WC5.4C $L$. ambigua SA

WC5.4d $L$. ambigua SA

WC5.4e $L$. ambigua SA

WC21.1i $L$. pauciflora SA

WC21.1b $L$. pauciflora SA

WC21.1j L. pauciflora SA

WC26.1d $L$. meyerianna SA

WC26.1e $L$. meyerianna SA

WC26.1f $L$. meyerianna SA

$P$. lemoignei (LMG2207)

R. detusculanense (APF11)

$R$. solanacearum (R221)

R. pickettii (ATCC27512)

Az. caulinodans (LMG6465)

P. aeruginosa (ATCC10145)

K. pneumoniae (AU45)

B. andropogonis (ATCC23061) T

$B$. fungorum (LMG19076) T

B. caribiensi (MWAP64)T

B. caryophylii (ATCC25418) T

B. cepacia (ATCC25416) T

B. cocovenenas (АTCC33664) T aagtcgaacg gtaacaggga g--ct--tg- -ctccgctga cgagtggcga aagtcgaacg gcagcacggg a--gc--aa- -tcctggtgg cgagtggcga aagtcggacg gcagcgcggg g--gc--aa- -ccctggcgg cgagtggcga aagtcgaacg gcagcacggg $9--9 c--a a-$-ccctggtgg cgagtggcga aagtcgaacg gcagcacggg t--gcttgc- -acctggtgg cgagtggcga aagtcgaacg gcagcacggg t--gcttgc- -acctggtgg cgagtggcga cattcgaacg gcagcacggg t--gcttgc- -acctggtgg cgagtggcga aagtcgaacg gcagcacggg g--gc--aa- -ccctggtgg cgagtggcga aagtcgaacg gcagcacggg t--gcttgc- -acctggtgg cgagtggcga aagtcgaacg gcagcacggg $9--9 c--a a-$-ccctggtgg cgagtggcga aagtcgaacg gcagcacggg g--gc--aa- -ccctggtgg cgagtggcga aagtcgaacg gcagcacggg $9--9 c--a a-$-ccctggtgg cgagtggcga aagtcgaacg gcagcacggg t--gcttgc- -acctggtgg cgagtggcga aattcgaacg gcagcacggg a--gc--aa- -tcctggtgg cgattggcga aagtcgaacg gcagcacggg $9--9 c--a a-$-ccctggtgg cgagtggcga aagtcgaacg gcagcacgg- ---gcttcg- -gcctggtgg cgagtggtga aagtcgaacg gcagcacggg t--gcttgc- -acctggtgg cgagtggcga aagtcgaacg gcagcacggg t--gcttgc- -acctggtgg cgagtggcga -agtcgaacg gcagcacggg g--gc--aa- -ccctggtgg cgagtggcga aagtcggacg gcagcgcggg c--tt--cg- -gcctggcgg cgagtggcga aagtcggacg gcagcgcggg c--tt--cg- -gcctggcgg cgagtggcga aagtcgaacg gcagcacggg $9--g c--a a c$-ccctggtgg cgagtggcga aagtcgaacg gcagcatgat ctagcttgct agattgatgg cgagtggcga aagtcgaacg gcagcacgg- ---gcttcg- -gcctggtgg cgagtggcga ---cgaacg gcagcacggg t--gcttgc- -acctggtgg cgagtggcga aagtcgaacg gcagcacggg t--gcttgc- -acctggtgg cgagtggcga aagtcgaacg gcagcacggg t--gcttgc- -acctggtgg cgagtggcga aagtcggacg gcagcgcgg- ---gcttcg- -gcctggcgg cgagtggcga aagtcgaacg gcagcggggg t-agcttgct -acctgccgg cgagtggcga aagtcgaacg gcagcacggg g--gc--aa- -ccctggtgg cgagtggcga aagtcgaacg gcagcacggg t--gcttgc- -acctggtgg cgagtggcga aagtcgaacg gcagcgcggg g--gc--aa- -ccctggcgg cgagtggcga aagtcgaacg gcagcacggg g--gc--aa- -ccctggtgg cgagtggcga aagtcgaacg gcagcgcgg- ---gcttcg- -gcctggcgg cgagtggcga aagtcgaacg gcagcacggg t--gcttgc- -acctggtgg cgagtggcga aagtcgaacg gcagcacgga c--tt--cg- -gtctggtgg cgagtggcga aagtcgaacg gcagcacggg t--gcttgc- -acctggtgg cgagtggcga

aagtcgaacg gcagcacggg t--gcttgc- -acctggtgg cgagtggcga aagtcgaacg gcagcacggg t--gcttgc- -acctggtgg cgagtggcga aagtcgaacg gcagcacggg t--gcttgc- -acctggtgg cgagtggcga aagtcgaacg gcagcacggg t--gcttgc- -acctggtgg cgagtggcga aagtcgaacg gcagcacggg $9--g c--a a-$-ccctggtgg cgagtggcga

aagtcggacg gcagcgcggg a--gc--aa- -tcctggcgg cgagtggcga aagtcgaacg gcagcacggg t--gcttgc- -acctggtgg cgagtggcga aagtcgaacg gcagcacggg t--gcttgc- -acctggtgg cgagtggcga aagtcgaacg gcagcacggg t--gcttgc- -a-ctggtgg cgagtggcga cagtcgaacg gcagcacgga c--tt--cg- -gtctggtgg cgagtggcga aagtcgaacg gcagcacggg g--gc--aa- -ccctggtgg cgagtggcga cagtcgaacg gcagcacggg g--gc--aa- -ccctggtgg cgagtggcga aagtcggacg gcagcacgga $\mathrm{c}--$ tt--cg- -gtctggtgg cgagtggcgaagtcggacg gcagcacgga c--tt--cg- -gtctggtgg cgagtggcgaagtcggacg gcagcacggg g--gc--aa- -ccctggtgg cgagtggcga aagtcggacg gcagcacggg g--gc--aa- -ccctggtgg cgagtg--gg aagtcggacg gcagcacggg $9--9 c--a a-$-ccctggtgg cgagtggcga cagtcggacg gcagcacggg g--gc--aa- -ccctggtgg cgagtggcga aagtcgaacg gcagcacggg t--rcttgc- -acctggtgg cgagtggcga aagtcgaacg gcagcatgat ctagcttgct agattgatgg cgagtggcga aagtcgaacg gcagcggggg t-agcttgc- -tacctccgg cgagtggcga aagtcgaacg gcagcatgat ctagcttgct agattgatgg cgagtggcga aagtcgagcg CC-------- --------- --cagcaatg ggagcggcag aagtcgagcg gatgaaggga ---gcttgc- --tcctggat tcagcggcgg aagtcgagcg gtagcacaga ga-gcttgct -ctcgggtga cgagcggcgg$$
70
$$

80

90

100 acgggtgagt aatacatcgg aacgtgtcct gtagtggggg atag-cccgg acgggtgagt aatacatcgg aacgtgtcct gtagtggggg atag-cccgg acgggtgagt aatacatcgg aacgtgtcct ggagtggggg atag--ccgg acgggtgagt aatacatcgg aacgtgtcct ggagtggggg atag-cccgg acgggtgagt aatacatcgg aacatgtcct gtagtggggg atag-cccgg acgggtgagt aatacatcgg aacatgtcct gtagtggggg atag-cccgg 
B. cocovenenas (LMG11626) T

$B$. fungorum (LMG16225)

B. gladioli (ATCC10248) T

B. glathei (LMG14190)T

B. glathei (ATCC29195) T

B. glathei (ATCC29195)T

B. glumae (LMG2196) T

B. graminis (C4D1M) T

B. hospita (LMG20598) T

B. mallei (ATCC23344)T

B. multivorans (LMG13010) T

B. norimbergensis (Y09879) T

B. phenazinium (LMG2247) T

B. phenoliruptrix (AC1100) T

B. phymatum (STM815) T

B. phytofirmans (PsJN) T

B. pickettii (ATCC27511)T

B. pseudomallei (ATCC23343) T

B. pyrrocinia (LMG14191) T

B. pyrrocinia ATCC15958)T

B. sacchari (AF263278)T

B. silvatlantica (SRMrh-20) T

B. solanacearum (ATCC11696) T

B. sordicola (S5-B) T

B. stabilis (LMG14294)T

B. terrae (KMY02) T

B. terriccola (LMG20594) T

B. thailandensis (E264)T

B. tropicalis (Ppe8) T

B. tuberum (STM678) T

B. unamae (MT1-641) T

B. vandii (LMG16020) T

B. vietnamiensis (AMMD) T

B. vietnamiensis (TVV75) T

B. vietnamiensis (TVV70) T

B. plantarii (LMG9035) T

B. xenovorans (LB400) T

B. cenocepacia (KN9) T

B. ferrariae (FeG101) T

B. ambifaria (LMG19182) T

B. oklahomensis (C6786) T

B. ubonensis (GTC-P3-415) T

WC5.4C $L$. ambigua SA

WC5.4d $L$. ambigua SA

WC5.4e L. ambigua SA

WC21.1i $L$. pauciflora SA

WC21.1b L. pauciflora SA

WC21.1j L. pauciflora SA

WC26.1d L. meyerianna SA

WC26.1e L. meyerianna SA

WC26.1f $L$. meyerianna SA

P. lemoignei (LMG2207)

$R$. detusculanense (APF11)

$R$. solanacearum (R221)

R. pickettii (ATCC27512)

Az. Caulinodans (LMG6465)

$P$. aeruginosa (ATCC10145)

$K$. pneumoniae (AU45)

B. andropogonis (ATCC23061) T

$B$. fungorum (LMG19076) T

B. caribiensi (MWAP64) T

B. caryophylii (ATCC25418) T

B. cepacia (ATCC25416) T

B. cocovenenas (ATCC33664) T

B. cocovenenas (LMG11626) T

$B$. fungorum (LMG16225)

B. gladioli (ATCC10248)T

B. glathei (LMG14190) T

B. glathei (ATCC29195) T

B. glathei (ATCC29195) T

B. glumae (LMG2196) T

B. graminis (C4D1M) T

B. hospita (LMG20598) T

B. mallei (ATCC23344)T

B. multivorans (LMG13010) T acgggtgagt aatacatcgg aacatgtcct gtagtggggg atag-cccgg acgggtgagt aatacatcgg aacgtgtcct gtagtggggg atag-cccgg acgggtgagt aatacatcgg aacatgtcct gtagtggggg atag-cccgg acgggtgagt aatacatcgg aacgtgtcct gtagtggggg atag-cccgg acgggtgagt aatacatcgg aacgtgtcct gtagtggggg atag--ccgg acgggtgagt aatacatcgg aacgtgtcct gtagtggggg atagccccg acgggtgagt aatacatcgg aacatgtcct gtagtggggg atag-cccgg acgggtgagt aatacatcgg aacgtgtcct gtagtggggg atag-cccgg acgggtgagt aatacatcgg aacgtgtcct ggagtggggg atag-cccgg acgggtgagt aatacatcgg aacatgtcct gtagtggggg atag-cccgg acgggtgagt aatacatcgg aacatgtcct gtagtggggg atag-cccgg acgggtgagt aatacatcgg aatgtacctt gtagtggggg atag-ctcgg acgggtgagt aatacatcgg aacgtgtcct gtagtggggg atag-cccgg acgggtgagt aatacatcgg aacgtgtcct gtagtggggg atag-cccgg acgggtgagt aatacatcgg aacgtgtcct ggagtggggg atag-cccgg acgggtgagt aatacatcgg aacgtgtcct gtagtggggg atag-cccgg acgggtgagt aatacatcgg aacgtgccct gtagtggggg ataa-ctagt acgggtgagt aatacatcgg aacatgtcct gtagtggggg atag-cccgg acgggtgagt aatacatcgg aacatgtcct gtagtggggg atag-cccgg acgggtgagt aatacatcgg aacatgtcct gtagtggggg atag-cccgg acgggtgagt aatacatcgg aacatgtcca gtagtggggg atag-cccgg acgggtgagt aatacatcgg aacatgtcca gtagtggggg atag-cccgg acgggtgagt aatacatcgg aacgtgccct gtagtggggg ataa-ctagt acgggtgagt aatacatcgg aacgtgtcct gtagtggggg atag-cccgg acgggtgagt aatacatcgg aacatgtcct gtagtggggg atag-cccgg acgggtgagt aatacatcgg aacgtgtcct ggagtggggg atag-cccgg acgggtgagt aatacatcgg aacgtgtcct gtagtggggg atag-cccgg acgggtgagt aatacatcgg aacatgtcct gtagtggggg atag-cccgg acgggtgagt aatacatcgg aacgtgtcct gtagtggggg atag-cccgg acgggtgagt aatacatcgg aacgtgtcct ggagtggggg atag-cccgg acgggtgagt aatacatcgg aacatgtcca gtagtggggg atag-cccgg -gatt aatacatcgg aacatgtcct gtactggggg atag-cccgg acgggtgagt aatacatcgg aacatgtcct gtagtggggg atag-cccgg acgggtgagt aatacatcgg aacatgtcct gtagtggggg atag-cccgg acgggtgagt aatacatcgg aacatgtcct gtagtggggg atag-cccgg acgggtgagt aatacatcgg aacatgtcct gtagtggggg atag-cccgg acgggtgagt aatacatcgg aacgtgtcct gtagtggggg atag-cccgg -----tgagt aatacatcgg aacatgtcct gtagtggggg atag-cccgg acgggtgagt aatacatcgg aacatgtcca gtagtggggg atag-cccgg acgggtgagt aatacatcgg aacatgtcct gtagtggggg atag-cccgg acgggtgagt aatacatcgg aacatgtcct gtagtggggg atag-cccgg acgggtgagt aatacatcgg aacatgtcct gtagtggggg atag-cccgg a-gttggagt aatacatcgg aacgtgtcct ggagtggggg atag-cccgg acgggtgagt aatacatcgg aacgtgtcct gtagtggggg atag-cccgg acgtgggagt aatacatcgg aacgtgtcct gtagtggggg atag-cccgg acgtgtgagt aatacatcgg aacgtgtcct ggagtggggg atag-cccgg acgggagagt aatacatcgg aacgtgtcct ggagtggggg atag-cccgg cc--gttgag tatacatcgg aacgtgtcct ggagtggggg actg-gccgg ccgtttaagt cctacatcgg aacgtgtcct ggagtggggg acg--tccgg accggtta-- tatacatcgg aacgtgtcct ggagtggggg atag-ccttg acgggttagt aatacatcgg aacgtgtcct ggagtggggg atag-cccgg acgggtgagt aatatatcgg aacgtgccca atagtggggg ataa-cgtag acgggtgagt aatacatcgg aacgtgccct gtagtggggg ataa-ctagt acgggtgagt aatacatcgg aacgtgccct gtagtggggg ataa--ctat acgggtgagt aatacatcgg aacgtgccct gtagtggggg ataa-ctagt acgggtgagt aacgcgtggg gatgtgccca atggtgcgga ataa-cccag acgggtgagt aatgcctagg aatctgcctg gtagtggggg ataa-cgtcc acgggtgagt aatgtctggg aaactgcctg atggaggggg ataa-ctact

$$
110
$$

130

140

150

cgaaagc--- attaataccg catacgatct aaggatgaaa gcgggggatc cgaaagccgg attaataccg catacgctct gcggaggaaa gcgggggatc cgaaagccgg attaataccg catacgctct gtggaggaaa gcgggggatc cgaaagc--- attaataccg catacgctcg ggagaggaaa gcgggggacc cgaaagccgg attaataccg catacgatcc acggatgaaa gcgggggacc cgaaagccgg attaataccg catacgatcc acggatgaaa gcgggggacc cgaaagccgg attaataccg catacgatcc acggatgaaa gcgggggacc cgaaagccgg attaataccg catacgctct acggaggaaa gggggggatc cgaaagc--- attaataccg catacgatct acggatgaaa gcgggggacc cgaaagccgg attaataccg catacgatct acggaagaaa gcgggggatc cgaaagccgg attaataccg catacgatct acggaagaaa gcgggggatc cgaaagccgg attaataccg catacgatct acggaagaaa gcgggggatc cgaaagccgg attaataccg catacgatct acggaagaaa gcgggggacc cgaaagccgg attaataccg catacgctct gcggaggaaa gcgggggatc cgaaagccgg attaataccg catacgatct ctggatgaaa gcgggggacc cgaaagccgg attaataccg catacgatct gaggatgaaa gcgggggacc cgaaagccgg attaataccg catacgatct acggatgaaa gcgggggacc 
B. norimbergensis (Y09879) T

B. phenazinium (LMG2247) T

B. phenoliruptrix (AC1100) T

B. phymatum (STM815) T

B. phytofirmans (PsJN) T

B. pickettii (ATCC27511)T

B. pseudomallei (ATCC23343) T

B. pyrrocinia (LMG14191) T

B. pyrrocinia ATCC15958)T

B. sacchari (AF263278)T

B. silvatlantica (SRMrh-20) T

B. solanacearum (ATCC11696) T

B. sordicola (S5-B) T

B. stabilis (LMG14294) T

B. terrae (KMY02) T

B. terriccola (LMG20594) T

B. thailandensis (E264)T

B. tropicalis (Ppe8) T

B. tuberum (STM678) T

B. unamae (MT1-641) T

B. vandii (LMG16020)T

B. vietnamiensis (AMMD) T

B. vietnamiensis (TVV75) T

B. vietnamiensis (TVV70) T

B. plantarii (LMG9035) T

B. xenovorans (LB400) T

B. cenocepacia (KN9) T

B. ferrariae (FeG101) T

B. ambifaria (LMG19182) T

B. oklahomensis (C6786) T

$B$. ubonensis (GTC-P3-415) T

WC5.4C L. ambigua SA

WC5.4d $L$. ambigua SA

WC5.4e $L$. ambigua SA

WC21.1i $L$. pauciflora SA

WC21.1b L. pauciflora SA

WC21.1j $L$. pauciflora SA

WC26.1d L. meyerianna SA

WC26.1e L. meyerianna SA

WC26.1f L. meyerianna SA

$P$. lemoignei (LMG2207)

$R$. detusculanense (APF11)

$R$. solanacearum (R221)

R. pickettii (ATCC27512)

Az. Caulinodans (LMG6465)

$P$. aeruginosa (ATCC10145)

$K$. pneumoniae (AU45)

B. andropogonis (ATCC23061) T

$B$. fungorum (LMG19076) T

B. caribiensi (MWAP64) T

B. caryophylii (ATCC25418) T

B. cepacia (ATCC25416) T

B. cocovenenas (ATCC33664) T

B. cocovenenas (LMG11626) T

$B$. fungorum (LMG16225)

B. gladioli (ATCC10248) T

B. glathei (LMG14190)T

B. glathei (ATCC29195) T

B. glathei (ATCC29195)T

B. glumae (LMG2196) T

B. graminis (C4D1M) T

B. hospita (LMG20598) T

B. mallei (ATCC23344)T

B. multivorans (LMG13010) T

$B$. norimbergensis (Y09879) T

B. phenazinium (LMG2247) T

B. phenoliruptrix (AC1100) T

B. phymatum (STM815) T

B. phytofirmans (PsJN) T

B. pickettii (ATCC27511)T

B. pseudomallei (ATCC23343) T

B. pyrrocinia (LMG14191) T

B. pyrrocinia ATCC15958) T

B. sacchari (AF263278) T

B. silvatlantica (SRMrh-20) T cgaaagccgg attaataccg catacgctct gaggaggaaa gcgggggacc cgaaagccgg attaataccg catacgctct acggaggaaa gggggggatc cgaaagccgg attaataccg catacgctct gcggaggaaa gcgggggatc cgaaagccgg attaataccg catacgctct gaggaggaaa gcgggggatc cgaaagccgg attaataccg catacgatct gtggatgaaa gcgggggatc cgaaagatta gctaataccg catacgacct gagggtgaaa gtgggggacc cgaaagccgg attaataccg catacgatct raggatgaaa gcgggggacc cgaaagccgg attaataccg catacgatct acggatgaaa gcgggggacc cgaaagc--- -ttaataccg catacgatct acggatgaaa gcgggggacc cgaaagccgg attaataccg catacgatct ttggatgaaa gcgggggacc cgaaagccgg attaataccg catacgctct gtggaggaaa gcgggggatc cgaaagactg gctaataccg catacgacct gagggtgaaa gtgggggacc cgaaagccgg attaataccg catacgatct acggaagaaa gcgggggacc cgaaagccgg attaataccg catacgatct ayggatgaaa gcgggggacc cgaaagccgg attaataccg catacgatct tgggatgaaa gcgggggacc cgaaagccgg attaataccg catacgctct gcggaggaaa gcgggggctc cgaaagccgg attaataccg catacgatct gtggatgaaa gcgggggacc cgaaagccgg attaataccg catacgatct acggatgaaa gcgggggatc cgaaagccgg attaataccg catacgctcg ggagaggaaa gcgggggatc cgaaagccgg attaataccg catacgatct acggatgaaa gcgggggacc cgaaagccgg attaataccg catacgatct acggatgaaa gcgggggatc cgaaagccgg attaataccg catacgatct acggatgaaa gcgggggacc cgaaagccgg attaataccg catacgatct atggatgaaa gcgggggacc cgaaagccgg attaataccg catacgatct atggatgaaa gcgggggacc cgaaagccgg attaataccg catacgatct acggatgaaa gcgggggatc cgaaagccgg attaataccg catacgctct gcggaggaaa gcgggggatc cgaaagccgg attaataccg catacgatct acggatgaaa gcgggggacc cgaaagccgg attaataccg catacgatct atggatgaaa gcgggggatc cgaaagccgg attaataccg catacgatct acggatgaaa gcgggggacc cgaaagccgg attaataccg catacgatct gaggatgaaa gcgggggacc cgaaagc--- attaataccg catacgatct acggatgaaa gcgggggacc cgaaagccgg attaataccg catacgctcg ggagaggaaa gcgggggatc cgaaagccgg attaataccg catacgctct gcggaggaaa gcgggggatc cgaaagccgg attaataccg catacgctct gcggaggaaa gcgggggatc cgaaagccgg attaataccg catacgctct gaggaggaaa gcgggggatc cgaaagccgg attaataccg catacgctct gaggaggaaa gcgggggatc cgaaagccgg attaataccg catacgctct gaggaggaaa gcgggggatc cgaaagccgg attaataccg catacgctct gaagaggaaa gcgggggatc cgaaagccgg attaataccg catacgctct gaagaggaaa gcgggggatc cgaaagccgg attaataccg catacgctct gaagaggaaa gcgggggatc cgaaagttac gctaataccg catacgctct acggaggaaa gtgggggatc cgaaagatta gctaataccg catacgacct gagggtgaaa gtgggggacc cgaaagactg gctaataccg catacgacct gagggtgaaa gtgggggacc cgaaagatta gctaataccg catacgacct gagggtgaaa gtgggggacc ggaaacttgg attaataccg catgtgccct tcgggggaaa

ggaaacgggc gctaataccg catacgtcct gagggagaaa gtgggggatc ggaaacggta gctaataccg cataatgtcg caagaccaaa gtgggggacc

160

170

180

190

200

--ttcggacc tcocgctata ggg-gcggcc gatggcagat tagctagttg cttcgggacc tcgcgctaca ggg-gcggcc gatggcagat tagctagttg --ttcggacc tcgcgctcaa ggg-gcggcc gatggcagat tagctagttg --ttcgggcc tcgcgctcaa ggg-gcggcc gatggcggat tagctagttg --ttcgggcc tcgcgctata ggg-ttggcc gatggctgat tagctagttg --ttcgggcc tcgcgctata ggg-ttggcc gatggctgat tagctagttg --ttcgggcc tcgcgctata ggg-ttggcc gatggctgat tagctagttg --ttaggacc tcccgctaca ggg-gcggcc gatggcagat tagctagttg --ttcgggcc tcgcgctata ggg-ttggcc gatggctgat tagctagttg --ttcggacc tcgcgctata ggg-gcggcc gatggcagat tagctagttg --ttcggacc tcgcgctata ggg-gcggcc gatggcagat tagctagttg --ttcggacc tcgcgc---- ggg-gcggcc gatggcagat tagctagttg --ttcgggcc tcgcgctata ggg-ttggcc gatggctgat tagctagttg cttcgggacc tcgcgctaca ggg-gcggcc gatggcagat tagctagttg --gaaaggcc tcgcgctcaa ggg-gcggcc gatggcagat tagctagttg --ttcgggcc tcgcgctata ggg-ttggcc gatggctgat tagctagttg --ttcgggcc tcgcgctata ggg-ttggcc gatggctgat tagctagttg --ttcgggcc tcgtgctaca aga-gcagcc gatgtcagat tagcttgttg --ttaggacc tctcgctaca ggg-gcggcc gatggcagat tagctagttg cttcgggacc tcgcgctaca ggg-gcggcc gatggcagat tagctagttg --ttcggacc tcgcgctcaa ggg-gcggcc gatggcagat tagctagtt --ttaggacc tcgcgctaca ggg-gcggcc gatggcagat tagctagttg --gcaaggcc tcatgctata gga-gcggcc gatgtctgat tagctagttg --ttcgggcc tcgcgctata ggg-ttggcc gatggctgat tagctagttg --ttcgggcc tcgcgctata ggg-ttggcc gatggctgat tagctagttg --ttcgggcc tcgcgcta-a ggg-ttggcc gatggctgat tagctagttg --ttcgggcc tcgcgctatt ggg-gtggcc gatggcggat taggtagttg --gaaagacc tcgcgctatt ggg-gtggcc gatggcggat tagctagatg 
B. solanacearum (ATCC11696) T

B. sordicola (S5-B) T

B. stabilis (LMG14294)T

$B$. terrae (KMY02) T

B. terriccola (LMG20594) T

$B$. thailandensis (E264) T

B. tropicalis (Ppe8) T

B. tuberum (STM678) T

B. unamae (MT1-641) T

B. vandii (LMG16020) T

$B$. vietnamiensis (AMMD) T

B. vietnamiensis (TVV75) T

B. vietnamiensis (TVV70)T

B. plantarii (LMG9035) T

B. xenovorans (LB400) T

B. cenocepacia (KN9) T

$B$. ferrariae (FeG101) T

B. ambifaria (LMG19182) T

B. oklahomensis (C6786) T

B. ubonensis (GTC-P3-415) T

WC5.4C $L$. ambigua SA

WC5.4d $L$. ambigua SA

WC5.4e $L$. ambigua SA

WC21.1i $L$. pauciflora SA

WC21.1b $L$. pauciflora SA

WC21.1j $L$. pauciflora SA

WC26.1d $L$. meyerianna SA

WC26.1e $L$. meyerianna SA

WC26.1f $L$. meyerianna SA

$P$. lemoignei (LMG2207)

$R$. detusculanense (APF11)

$R$. solanacearum (R221)

R. pickettii (ATCC27512)

Az. caulinodans (LMG6465)

$P$. aeruginosa (ATCC10145)

$K$. pneumoniae (AU45)

B. andropogonis (ATCC23061) T

$B$. fungorum (LMG19076) T

B. caribiensi (MWAP64) T

B. caryophylii (ATCC25418)T

B. cepacia (ATCC25416) T

B. cocovenenas (ATCC33664)T

B. cocovenenas (LMG11626) T

$B$. fungorum (LMG16225)

B. gladioli (ATCC10248) T

B. glathei (LMG14190) T

B. glathei (ATCC29195)T

B. glathei (ATCC29195) T

B. glumae (LMG2196) T

B. graminis (C4D1M) T

B. hospita (LMG20598) T

B. mallei (ATCC23344) T

B. multivorans (LMG13010) T

$B$. norimbergensis (Y09879) T

B. phenazinium (LMG2247) T

B. phenoliruptrix (AC1100) T

B. phymatum (STM815) T

B. phytofirmans (PsJN) T

B. pickettii (ATCC27511) T

B. pseudomallei (ATCC23343) T

B. pyrrocinia (LMG14191) T

B. pyrrocinia ATCC15958) T

B. sacchari (AF263278) T

$B$. silvatlantica (SRMrh-20)T

B. solanacearum (ATCC11696) T

B. sordicola (S5-B) T

B. stabilis (LMG14294) T

B. terrae (KMY02) T

B. terriccola (LMG20594) T

B. thailandensis (E264) T

B. tropicalis (Ppe8) T

B. tuberum (STM678) T

B. unamae (MT1-641) T

B. vandii (LMG16020) T

$B$. vietnamiensis (AMMD) T --gcaaggcc tcatgctata gga-gcggcc gatgtctgat tagctagttg --ttagggcc tcgcgctata ggg-gcggcc gatggcagat tagctagttg --ttcgggcc tcgcgctata ggg-ttggcc gatggctgat tagctagttg --gaaaggcc tcgcgctcaa ggg-gcggcc gatggcagat tagctagttg cttcgggacc tcgcgctaca ggg-gcggcc gatggcagat tagctagttg --ttcgggcc tcgcgctata ggg-ttggcc gatggctgat tagctagttg --ttcggacc tcgcgctata ggg-gcggcc gatggcggat tagctagttg tttcgggacc tcgcgctcaa ggg-gcggcc gatggcagat taggtagttg --ttcgggcc tcgcgctatt ggg-gtggcc gatggcggat tagctagttg --ttcggacc tcgcgctata ggg-ttggcc gatggctgat tagctagttg --ttcgggcc tcgcgctata ggg-ttggcc gatggctgat tagctagttg --ttcgggce tcgcgctata ggg-ttggce gatggctgat tagctagttg --ttcgggcc tcgcgctata ggg-ttggcc gatggctgat tagctagttg --tttggacc tcgcgctata ggg-ttggce gatggctgat tagctagttg --ttcggacc tcgcgctaca ggg-gcggcc gatggcagat tagctggttg --ttcgggce tcgcgctata ggg-ttggcc gatggctgat tagctagttg --gcaagacc tcgcgctatt ggg-gtggcc gatggcggat tagctagttg --ttcgggcc tcgcgctata ggg-ttggcc gatggctgat tagctagttg --ttcgggcc tcgcgctata ggg-ttggcc gatggctgat tagctagttg --ttcgggcc tcgcgctata ggg-ttggcc gatggctgat tagctagttg tttcgggacc tcgcgctcaa ggg-gcggcc gatggcagat taggtagttg --ttaggacc tcgcgctaca ggg-gcggcc gatggcagat tagctagttg --ttaggacc tcgcgctaca ggg-gcggcc gatggcagat tagctagttg tttcgggacc tcgcgctcaa ggg-gcggcc gatggcagat taggtagttg tttcgggacc tcgcgctcaa ggg-gcggcc gatggcagat taggtagttg tttcgggacc tcgcgctcaa ggg-gcggcc gatggcagat taggtagtto tttcgggacc tcgcgctcaa ggg-gcggcc gatggcagat taggtagttg tttcgggacc tcgcgctcaa ggg-gcggcc gatggcagat taggtagtto tttcgggacc tcgcgctcaa ggg-gcggcc gatggcagat taggtagttg --gcaagacc tcatgctatt gga-gcggcc gatatctgat tagctagttg --gcaaggcc tcatgctata gga-gcggcc gatgtctgat tagctagttg --gcaaggcc tcatgctata gga-gcggcc gatgtctgat tagctagttg --gcaaggcc tcatgctata gga-gcggcc gatgtctgat tagctagttg ------gatt tatcgccatt gga-tcaacc cgcgtctgat tagctagttg --ttcggacc tcacgctatc aga-tgagcc taggtcggat tagctagttg --ttcgggcc tcatgccatc aga-tgtgcc cagatgggat tagctagtag

210 220 230 240 250 gtggggtaaa ggcctaccaa ggcgacgatc cgtagctggt ctgagaggac gtggggtaaa ggcctaccaa ggcgacgatc tgtagctggt ctgagaggac gtggggtaaa ggcctaccaa ggcgacgatc tgtagctggt ctgagaggac gtggggtaaa ggcctaccaa ggcgacgatc cgtagctggt ctgagaggac gtggggtaaa ggcctaccaa ggcgacgatc agtagctggt ctgagaggac gtggggtaaa ggcccaccaa ggcgacgatc agtagctggt ctgagaggac gtggggtaaa ggcccaccaa ggcgacgatc agtagctggt ctgagaggac gtggggtaaa ggcctaccaa ggcgacgatc tgtagctggt ctgagaggac gtggggtaaa ggcccaccaa ggcgacgatc agtagctggt ctgagaggac gtggggtaaa ggcctaccaa ggcgacgatc tgtagctggt ctgagaggac gtggggtaaa ggcctaccaa ggcgacgatc tgtagctggt ctgagaggac gtggggtaaa ggcctaccaa ggcgacgatc tgtagctggt ctgagaggac gtggggtaaa ggcctaccaa ggcgacgatc agtagctggt ctgagaggac gtggggtaaa ggcctaccaa ggcgacgatc tgtagctggt ctgagaggac gtggggtaaa ggcctaccaa ggcgacgatc tgtagctggt ctgagaggac gtggggtaaa ggcctaccaa ggcgacgatc agtagctggt ctgagaggac gtggggtaaa ggcctaccaa ggcgacgatc agtagctggt ctgagaggac gtgaggtaaa agctcaccaa ggcgacgatc tgtagctggt ctgagaggac gtggggtaaa ggcctaccaa ggcgacgatc tgtagctggt ctgagaggac gtggggtaaa ggcctaccaa ggcgacgatc tgtagctggt ctgagaggac gtggggtaaa ggcctaccaa ggcgacgatc tgtagctggt ctgagaggac gtggggtaaa ggcctaccaa ggcgacgatc tgtagctggt ctgagaggac gtgaggtaaa ggctcaccaa ggcgacgatc agtagctggt ctgagaggac gtggggtaaa ggcctaccaa ggcgacgatc agtagctggt ctgagaggac gtggggtaaa ggcctaccaa ggcgacgatc agtagctggt ctgagaggac gtggggtaaa ggcctaccaa ggcgacgatc agtagctggt ctgagaggac gtggggtaaa ggcctaccaa gccgacgatc cgtagctggt ctgagaggac gtggggtaaa ggcctaccat ggcgacgatc cgtagctggt ctgagaggac gtggggtaaa ggcctaccaa ggcgacgatc agtagctggt ctgagaggac gtggggtaaa ggcctaccaa ggcgacgatc tgtagctggt ctgagaggac gtggggtaaa ggcctaccaa ggcgacgatc agtagctggt ctgagaggac gtggggtaaa ggcctaccaa ggcgacgatc tgtagctggt ctgagaggac gtggggtaaa ggcctaccaa ggcgacgatc tgtagctggt ctgagaggac gtggggtaaa ggcctaccaa ggcgacgatc agtagctggt ctgagaggac gtgaggtaaa ggctcaccaa ggcgacgatc cgtagctggt ctgagaggac gtggggtaaa ggcctaccaa gccgacgatc tgtagctggt ctgagaggac gtggggtaaa ggctcaccaa ggcgacgatc cgtagctggt ctgagaggac gtggggtaaa ggcctaccaa ggcgacgatc agtagctggt ctgagaggac gtggggtaaa ggcctaccaa ggcgacgatc agtagctggt ctgagaggac 
B. vietnamiensis (TVV75) T

$B$. vietnamiensis (TVV70) T

B. plantarii (LMG9035) T

B. xenovorans (LB400) T

$B$. cenocepacia (KN9) T

B. ferrariae (FeG101) T

B. ambifaria (LMG19182) T

B. oklahomensis (C6786) T

B. ubonensis (GTC-P3-415) T

WC5.4C $L$. ambigua SA

WC5.4d $L$. ambigua SA

WC5.4e $L$. ambigua SA

WC21.1i L. pauciflora SA

WC21.1b $L$. pauciflora SA

WC21.1j $L$. pauciflora SA

WC26.1d $L$. meyerianna SA

WC26.1e $L$. meyerianna SA

WC26.1f $L$. meyerianna SA

$P$. lemoignei (LMG2207)

$R$. detusculanense (APF11)

$R$. solanacearum (R221)

R. pickettii (ATCC27512)

Az. caulinodans (LMG6465)

$P$. aeruginosa (ATCC10145)

$K$. pneumoniae (AU45)

B. andropogonis (ATCC23061) T

$B$. fungorum (LMG19076) $T$

B. caribiensi (MWAP64) T

B. caryophylii (ATCC25418) T

B. cepacia (ATCC25416) T

B. cocovenenas (ATCC33664)T

$B$. cocovenenas (LMG11626) T

$B$. fungorum (LMG16225)

B. gladioli (ATCC10248) T

B. glathei (LMG14190) T

B. glathei (ATCC29195)T

B. glathei (ATCC29195) T

B. glumae (LMG2196) T

B. graminis (C4D1M) T

B. hospita (LMG20598) T

B. mallei (ATCC23344) T

B. multivorans (LMG13010) T

B. norimbergensis (Y09879) T

B. phenazinium (LMG2247)T

B. phenoliruptrix (AC1100) T

B. phymatum (STM815) T

B. phytofirmans (PsJN) T

B. pickettii (ATCC27511)T

B. pseudomallei (ATCC23343) T

B. pyrrocinia (LMG14191) T

B. pyrrocinia ATCC15958)T

B. sacchari (AF263278) T

B. silvatlantica (SRMrh-20)T

B. solanacearum (ATCC11696) T

$B$. sordicola (S5-B) $\mathrm{T}$

B. stabilis (IMG14294) T

B. terrae (KMY02) T

B. terriccola (LMG20594) T

B. thailandensis (E264) T

$B$. tropicalis (Ppe8) $\mathrm{T}$

B. tuberum (STM678) T

B. unamae (MT1-641) T

$B$. vandii (LMG16020) T

$B$. vietnamiensis (AMMD) T

B. vietnamiensis (TVV75) T

B. vietnamiensis (TVV70)T

B. plantarii (LMG9035) T

B. xenovorans (LB400) T

B. cenocepacia (KN9) T

B. ferrariae (FeG101) T

B. ambifaria (LMG19182) T

B. oklahomensis (C6786) T

B. ubonensis (GTC-P3-415) T

WC5.4C $L$. ambigua SA

WC5.4d L. ambigua SA gtggggtaaa ggcctaccaa ggcgacgatc agtagctggt ctgagaggac gtggggtaaa ggcctaccaa ggcgacgatc agtagctggt ctgagaggac gtggggtaaa ggcctaccaa ggcgacgatc agtagctggt ctgagaggac gtggggtaaa ggcctaccaa ggcgacgatc tgtagctggt ctgagaggac gtggggtaaa ggcctaccaa ggcgacgatc agtagctggt ctgagaggac gtggggtaaa ggcctaccaa ggcgacgatc cgtagctggt ctgagaggac gtggggtaaa ggcctaccaa ggcgacgatc agtagctggt ctgagaggac gtggggtaaa ggcctaccaa ggcgacgatc agtagctggt ctgagaggac gtggggtaaa ggcctaccaa ggcgacgatc agtagctggt ctgagaggac gtggggtaaa ggcctaccaa gccgacgatc tgtagctggt ctgagaggac gtggggtaaa ggcctaccaa ggcgacgatc tgtaggtggt ctgagaggac gtggggtaaa ggcctaccaa ggcgacgatc tgtagctggt ctgagaggac gtggggtaaa ggcctaccaa gccgacgatc tgtagctggt ctgagaggac gtggggtaaa ggcctaccaa gccgacgatc tgtagctggt ctgagaggac gtggggtaaa ggcctaccaa gccgacgatc tgtagctggt ctgagaggac gtggggtaaa ggcctaccaa gccgacgatc tgtagctggt ctgagaggac gtggggtaaa ggcctaccaa gccgacgatc tgtagctggt ctgagaggac gtggggtaaa ggcctaccaa gccgacgatc tgtagctggt ctgagaggac gtggggtaat ggcccaccaa ggcgacgatc agtagctggt ctgagaggac gtgaggtaaa ggctcaccaa ggcgacgatc agtagctggt ctgagaggac gtggggtaaa ggcccaccaa ggcgacgatc agtagctggt ctgagaggac gtgaggtaaa ggctcaccaa ggcgacgatc agtagctggt ctgagaggac gtgaggtaaa ggctcaccaa ggcgacgatc agtagctggt ctgagaggat gtggggtaaa ggcctaccaa ggcgacgatc cgtaactggt ctgagaggat gtggggtaac ggctcaccta ggcgacgatc cctagctggt ctgagaggat

260 270

280

290

300

gaccagccac actgggactg agacacggcc cagactccta c-gggaggca gaccagccac actgggactg agacacggcc cagactccta $\mathrm{c}-g$ ggaggca gaccagccac actgggactg agacacggcc cagactccta c-gggaggca gaccagccac actgggactg agacacggcc cagactccta c-gggaggca gaccagccac actgggactg agacacggcc cagactccta c-gggaggca gaccagccac actgggactg agacacggcc cagactccta c-gggaggca gaccagccac actgggactg agacacggcc cagactccta c-gggaggca gaccagccac actgggactg agacacggcc cagactccta c-gggaggca gaccagccac actgggactg agacacggcc cagactccta c-gggaggca gaccagccac actgggactg agacacggcc cagactccta c-gggaggca gaccagccac actgggactg agacacggcc cagactccta c-gggaggca gaccagccac actgggactg agacacggcc cagactccta c-gggaggca gaccagccac actgggactg agacacggcc cagactccta c-gggaggca gaccagccac actgggactg agacacggcc cagactccta c-gggaggca gaccagccac actgggactg agacacggcc cagactccta c-gggaggca gaccagccac actgggactg agacacggcc cagactccta c-gggaggca gaccagccac actgggactg agacacggcc cagactccta c-gggaggca gaccagccac actgggactg agacacggcc cagactccta c-gggaggca gaccagccac actgggactg agacacggcc cagactccta c-gggaggca gaccagccac actgggactg agacacggcc cagactccta c-gggaggca gaccagccac actgggactg agacacggcc cagactccta c-gggaggca gaccagccac actgggactg agacacggcc cagactccta c-gggaggca gatcagccac actgggactg agacacggcc cagactccta c-gggaggca gaccagccac actgggactg agacacggcc cagactccta c-gggaggca gaccagccac actgggactg agacacggcc cagactccta c-gggaggca gaccagccac actgggactg agacacggcc cagactccta c-gggaggca gaccagccac actgggactg agacacggcc cagactccta c-gggaggca gaccagccac actgggactg agacacggcc cagactccta c-gggaggca gatcagccac actgggactg agacacggcc cagactccta $\mathrm{c}-$ gggaggca gaccagccac actgggactg agacacggcc cagactccta c-gggaggca gaccagccac actgggactg agacacggcc cagactccta c-gggaggca gaccagccac actgggactg agacacggcc cagactccta c-gggaggca gaccagccac actgggactg agacacggcc cagactccta c-gggaggca gaccagccac actgggactg agacacggcc cagactccta c-gggaggca gaccagccac actgggactg agacacggcc cagactccta c-gggaggca gaccagccac actgggactg agacacggcc cagactccta c-gggaggca gaccagccac actgggactg agacacggcc cagactccta c-gggaggca gaccagccac actgggactg agacacggcc cagactccta c-gggaggca gaccagccac actgggactg agacacggcc cagactccta c-gggaggca gaccagccac actgggactg agacacggcc cagactccta c-gggaggca gaccagccac actgggactg agacacggcc cagactccta c-gggaggca gaccagccac actgggactg agacacggcc cagactccta c-gggaggca gaccagccac actgggactg agacacggcc cagactccta c-gggaggca gaccagccac actgggactg agacacggcc cagactccta c-gggaggca gaccagccac actgggactg agacacggcc cagactccta c-gggaggca gaccagccac actgggactg agacacggcc cagactccta cggggaagca gaccagccac actgggactg agacacggcc cagactccta c-gggaggca gaccagccac actgggactg agacacggcc cagactccta c-gggaggca gaccagccac actgggactg agacacggcc cagactccta c-gggaggca gaccagccac actgggactg agacacggcc cagactccta c-gggaggca 
WC5.4e $L$. ambigua SA WC21.1i L. pauciflora SA WC21.1b $L$. pauciflora SA WC21.1j $L$. pauciflora SA WC26.1d $L$. meyerianna SA WC26.1e $L$. meyerianna SA WC26.1f $L$. meyerianna SA $P$. lemoignei (LMG2207)

$R$. detusculanense (APF11)

$R$. solanacearum (R221)

R. pickettii (ATCC27512)

Az. caulinodans (LMG6465)

$P$. aeruginosa (ATCC10145)

$K$. pneumoniae (AU45)

B. andropogonis (ATCC23061) T

$B$. fungorum (LMG19076) $T$

B. caribiensi (MWAP64) T

B. caryophylii (ATCC25418) T

B. cepacia (ATCC25416) T

B. cocovenenas (ATCC33664) T

$B$. cocovenenas (LMG11626) T

$B$. fungorum (LMG16225)

B. gladioli (ATCC10248) T

B. glathei (LMG14190) T

B. glathei (ATCC29195)T

B. glathei (ATCC29195) T

B. glumae (LMG2196) T

$B$. graminis (C4D1M) T

B. hospita (LMG20598) T

B. mallei (ATCC23344) T

B. multivorans (LMG13010) T

B. norimbergensis (Y09879) T

B. phenazinium (LMG2247) T

B. phenoliruptrix (AC1100) T

B. phymatum (STM815) T

B. phytofirmans (PsJN) T

B. pickettii (ATCC27511)T

B. pseudomallei (ATCC23343) T

B. pyrrocinia (LMG14191) T

B. pyrrocinia ATCC15958) T

B. sacchari (AF263278) T

B. silvatlantica (SRMrh-20) T

B. solanacearum (ATCC11696) T

B. sordicola (S5-B) T

B. stabilis (LMG14294) T

B. terrae (KMY02) T

B. terriccola (LMG20594) T

$B$. thailandensis (E264) T

B. tropicalis (Ppe8) T

B. tuberum (STM678) T

B. unamae (MT1-641) T

$B$. vandii (LMG16020) T

$B$. vietnamiensis (AMMD) T

B. vietnamiensis (TVV75) T

$B$. vietnamiensis (TVV70)T

B. plantarii (LMG9035) T

B. xenovorans (LB400) T

$B$. cenocepacia (KN9) T

$B$. ferrariae (FeG101) T

B. ambifaria (LMG19182) T

$B$. oklahomensis (C6786) T

B. ubonensis (GTC-P3-415) T

WC5.4C $L$. ambigua SA

WC5.4d $L$. ambigua SA

WC5.4e $L$. ambigua SA

WC21.1i L. pauciflora SA

WC21.1b $L$. pauciflora SA

WC21.1j $L$. pauciflora SA

WC26.1d $L$. meyerianna SA

WC26.1e $L$. meyerianna SA

WC26.1f L. meyerianna SA

$P$. lemoignei (LMG2207)

$R$. detusculanense (APF11)

R. solanacearum (R221)

R. pickettii (ATCC27512) gaccagccac actgggactg agacacggcc cagactccta c-gggaggca gaccagccac actgggactg agacacggcc cagactccta c-gggaggca gaccagccac actgggactg agacacggcc cagactccta $\mathrm{c}-g$ ggaggca gaccagccac actgggactg agacacggcc cagactccta $\mathrm{c}-g$ ggaggca gaccagccac actgggactg agacacggcc cagactccta c-gggaggca gaccagccac actgggactg agacacggcc cagactccta c-gggaggca gaccagccac actgggactg agacacggcc cagactccta c-gggaggca gaccarccac actgggactg agacacggcc cagactccta c-gggaggca gatcagccac actgggactg agacacggcc cagactccta c-gggaggca gatcagccac actgggactg agacacggcc cagactccta c-gggaggca gatcagccac actgggactg agacacggcc cagactccta c-gggaggca gatcagccac actgggactg agacacggcc cagactccta c-gggaggca gatcagtcac actggaactg agacacggtc cagactccta c-gggaggca gaccagccac actggaactg agacacggtc cagactccta c-gggaggca

310 320 330 340 350 gcagtgggga attttggaca atgggcgcaa gcctgatcca gcaatgccgc gcagtgggga attttggaca atgggcgcaa gcctgatcca gcaatgccgc gcagtgggga attttggaca atgggggcaa ccctgatcca gcaatgccgc gcagtgggga attttggaca atgggggcaa ccctgatcca gcaatgccgc gcagtgggga attttggaca atgggcgaaa gcctgatcca gcaatgccgc gcagtgggga attttggaca atgggcgaaa gcctgatcca gcaatgccgc gcagtgggga attttggaca atgggcgaaa gcctgatcca gcaatgccgc gcagtgggga attttggaca atgggggcaa ccctgatcca gcaatgccgc gcagtgggga attttggaca atgggcgaaa gcctgatcca gcaatgccgc gcagtgggga attttggaca atgggggaaa ccctgatcca gcaatgccgc gcagtgggga attttggaca atgggggaaa ccctgatcca gcaatgccgc gcagtgggga attttggaca atgggggaaa ccctgatcca gcaatgccgc gcagtgggga attttggaca atgggcgaaa gcctgatcca gcaatgccgc gcagtgggga attttggaca atgggcgcaa gcctgatcca gcaatgccgc gcagtgggga attttggaca atgggggcaa ccctgatcca gcaatgccgc gcagtgggga attttggaca atgggcgcaa gcctgatcca gcaatgccgc gcagtgggga attttggaca atgggcgaaa gcctgatcca gcaatgccgc gcagtgggga attttggaca atgggcgaaa g-ctgatcca gcaatgccgc gcagtgggga attttggaca atgggggcaa ccctgatcca gcaatgccgc gcagtgggga attttggaca atgggggcaa ccctgatcca gcaatgccgc gcagtgggga attttggaca atgggggcaa ccctgatcca gcaatgccgc gcagtgggga attttggaca atgggcgcaa gcctgatcca gcaatgccgc gcagtgggga attttggaca atgggcgaaa gcctgatcca gcaatgccgc gcagtgggga attttggaca atgggcgcaa gcctgatcca gcaatgccgc gcagtgggga attttggaca atgggcgaaa gcctgatcca gcaatgccgc gcagtgggga attttggaca atgggcgaaa gcctgatcca gcaatgccgc gcagtgggga attttggaca atgggggaaa ccctgatcca gcaatgccgc gcagtgggga attttggaca atgggggcaa ccctgatcca gcaatgccgc gcagtgggga attttggaca atgggggcaa ccctgatcca gcaatgccgc gcagtgggga attttggaca atgggggcaa ccctgatcca gcaatgccgc gcagtgggga attttggaca atgggcgaaa gcctgatcca gcaatgccgc gcagtgggga attttggaca atgggggcaa ccctgatcca gcaatgccgc gcagtgggga attttggaca atgggcgcaa gcctgatcca gcaatgccgc gcagtgggga attttggaca atgggcgcaa gcctgatcca gcaatgccgc gcagtgggga attttggaca atgggcgaaa gcctgatcca gcaatgccgc gcagtgggga attttggaca atgggcgcaa gcctgatcca gcaatgccgc gcagtgggga attttggaca atgggcgaaa gcctgatcca gcaatgccgc gcagtgggga attttggaca atgggcgaaa gcctgatcca gcaatgccgc gcagtgggga attatggaca atgggcgaaa gcctgatcca gcaatgccgc gcagtgggga attttggaca atgggcgaaa gcctgatcca gcaatgccgc gcagtgggga attttggaca atgggcgaaa gcctgatcca gcaatgccgc gcagtgggga attttggaca atgggcgaaa gcctgatcca gcaatgccgc gcagtgggga attttggaca atgggcgcaa gcctgatcca gcaatgccgc gcagtgggga attttggaca atgggcgaaa gcctgatcca gcaatgccgc gcagtgggga attttggaca atgggggcaa ccctgatcca -caatgccgc gcagtgggga attttggaca atgggcgaaa gcctgatcca gcaatgccgc gcagtgggga attttggaca atgggcgcaa gcctgatcca gcaatgccgc gcagtgggga attttggaca atgggcg-aa -cctgatcca gcaatgccgc gcagtgggga attttggaca atgggcgcaa gcctgatcca gcaatgccgc gcagtgggga attttggaca atgggggcaa ccctgatcca gcaatgccgc gcagtgggga attttggaca atgggggcaa ccctgatcca gcaatgccgc gcagtgggga attttggaca atgggggcaa ccctgatcca gcaatgccgc gcagtgggga attttggaca atgggggcaa ccctgatcca gcaatgccgc gcagtgggga attttggaca atgggggcaa ccctgatcca gcaatgccgc gcagtgggga attttggaca atgggggcaa ccctgatcca gcaatgccgc gcagtgggga attttggaca atgggggcaa ccctgatcca gcaatgccgc gcagtgggga attttggaca atgggggcaa ccctgatcca gcaatgccgc gcagtgggga attttggaca atgggggcaa ccctgatcca gcaatgccgc gcagtgggga attttggaca atgggcgaaa gcctgatcca gcaatgccgc gcagtgggga attttggaca atgggggcaa ccctgatcca gcaatgccgc gcagtgggga attttggaca atgggcgaaa gcctgatcca gcaatgccgc 
Az. Caulinodans (LMG6465)

$P$. aeruginosa (ATCC10145)

$K$. pneumoniae (AU45)

B. andropogonis (ATCC23061) T

$B$. fungorum (LMG19076) T

B. caribiensi (MWAP64) T

B. caryophylii (ATCC25418) T

B. cepacia (ATCC25416) T

B. cocovenenas (ATCC33664)T

B. cocovenenas (LMG11626) T

$B$. fungorum (LMG16225)

B. gladioli (ATCC10248) T

B. glathei (LMG14190) T

B. glathei (ATCC29195)T

B. glathei (ATCC29195) T

B. glumae (LMG2196) T

$B$. graminis (C4D1M) T

B. hospita (LMG20598) T

B. mallei (ATCC23344) T

B. multivorans (LMG13010) T

B. norimbergensis (Y09879) T

B. phenazinium (LMG2247) T

B. phenoliruptrix (AC1100) T

B. phymatum (STM815) T

B. phytofirmans (PsJN) T

B. pickettii (ATCC27511) T

B. pseudomallei (ATCC23343) T

B. pyrrocinia (LMG14191)T

B. pyrrocinia ATCC15958) T

B. sacchari (AF263278) T

B. silvatlantica (SRMrh-20) T

B. solanacearum (ATCC11696)T

$B$. sordicola (S5-B) $\mathrm{T}$

B. stabilis (LMG14294) T

B. terrae (KMY02) T

B. terriccola (LMG20594) T

$B$. thailandensis (E264)T

B. tropicalis (Ppe8) T

B. tuberum (STM678) T

B. unamae (MT1-641) T

B. vandii (LMG16020) T

$B$. vietnamiensis (AMMD) T

B. vietnamiensis (TVV75) T

B. vietnamiensis (TVV70)T

B. plantarii (LMG9035) T

B. xenovorans (LB400) T

$B$. cenocepacia (KN9) T

$B$. ferrariae (FeG101) T

B. ambifaria (LMG19182) T

B. oklahomensis (C6786) T

B. ubonensis (GTC-P3-415) T

WC5.4C $L$. ambigua SA

WC5.4d $L$. ambigua SA

WC5.4e $L$. ambigua SA

WC21.1i L. pauciflora SA

WC21.1b $L$. pauciflora SA

WC21.1j $L$. pauciflora SA

WC26.1d $L$. meyerianna SA

WC26.1e $L$. meyerianna SA

WC26.1f $L$. meyerianna SA

$P$. lemoignei (LMG2207)

$R$. detusculanense (APF11)

$R$. solanacearum (R221)

R. pickettii (ATCC27512)

Az. Caulinodans (LMG6465)

$P$. aeruginosa (ATCC10145)

K. pneumoniae (AU45)

B. andropogonis (ATCC23061) T

B. fungorum (LMG19076) T

B. caribiensi (MWAP64) T

B. caryophylii (ATCC25418)T

B. cepacia (ATCC25416) T

B. cocovenenas (ATCC33664)T gcagtgggga atattggaca atgggcgcaa gcctgatcca gccatgccgc gcagtgggga atattggaca atgggcgaaa gcctgatcca gccatgccgc gcagtgggga atattgcaca atgggcgcaa gcctgatgca gccatgccgc

360

370

380

390

400

gtgtgtgaag aaggccttcg ggttgtaaag cacttttgtt cgggaagaaa gtgtgtgaag aaggccttcg ggttgtaaag cacttttgtc cggaaagaaa gtgtgtgaag aaggc-ttcg ggttgtaaag cacttttgtc cggaaagaaa gtgtgcgaag aaggccttcg ggttgtaaag cacttttgtc cggaaagaaa gtgtgtgaag aaggccttcg ggttgtaaag cacttttgtc cggaaagaa gtgtgtgaag aaggccttcg ggttgtaaag cacttttgtc cggaaagaa gtgtgtgaag aaggccttcg ggttgtaaag cacttttgtc cggaaagaaa gtgtgtgaag aaggccttcg ggttgtaaag cacttttgtc cggaaagaa gtgtgtgaag aaggccttcg ggttgtaaag cacttttgtc cggaaagaaa gtgtgtgaag aaggccttcg ggttgtaaag cacttttgtc cggaaagaaa gtgtgtgaag aaggccttcg ggttgtaaag cacttttgtc cggaaagaa gtgtgtgaac aaggccttcg ggttgtaaag cacttttgtc cggaaagaaa gtgtgtgaag aaggccttcg ggttgtaaag cacttttgtc cggaaagaaa gtgtgtgaag aaggccttcg ggttgtaaag cacttttgtc cggaaagaaa gtgtgtgaag aaggccttcg ggttgtaaag cacttttgtc cggaaagaaa gtgtgtgaag aaggccttcg ggttgtaaag cacttttgtc cggaaagaa gtgtgtgaag aaggccttcg ggttgtaaag cacttttgtc cggaaagaaa gtgtgtgaag aaggccttcg ggttgtaaag cacttttgtc cggaaagaaa gtgtgtgaag aaggccttcg ggttgtaaag cacttttgtc cggaaagaaa gtgtgtgaag aaggccttcg ggttgtaaag cacttttgtc cggaaagaaa gtgtgtgaag aaggccttcg ggttgtaaag cacttttgtc cggaaagaaa gtgtgtgaag aaggccttcg ggttgtaaag cacttttgtc cggaaagaa gtgtgtgaag aaggccttcg ggttgtaaag cacttttgtc cggaaagaaa gtgtgtgaag aaggccttcg ggttgtaaag cacttttgtc cggaaagaaa gtgtgtgaag aaggccttcg ggttgtaaag cacttttgtc cggaaagaa gtgtgtgaag aaggccttcg ggttgtaaag cacttttgtc cggaaagaaa gtgtgtgaag aaggccttcg ggttgtaaag cacttttgtc cggaaagaa gtgtgtgaag aaggccttcg ggttgtaaag cacttttgtc cggaaagaaa gtgtgtgaag aaggccttcg ggttgtaaag cacttttgtc cggaaagaaa gtgtgtgaag aaggccttcg ggttgtaaag cacttttgtc cggaaagaaa gtgtgtgaag aaggccttcg ggttgtaaag cacttttgtc cggaaagaaa gtgtgtgaag aaggccttcg ggttgtaaag cacttttgtc cggaaagaaa gtgtgtgaag aaggccttcg ggttgtaaag cacttttgtc cggaaagaaa gtgtgtgaag aaggccttcg ggttgtaaag cacttttgtc cggaaagaaa gtgtgtgaag aaggccttcg ggttgtaaag cacttttgtc cggaaagaaa gtgtgtgaag aaggccttcg ggttgtaaag cacttttgtc cggaaagaa gtgtgtgaag aaggccttcg ggttgtaaag cacttttgtc cggaaagaaa gtgtgtgaag aaggccttcg ggttgtaaag cacttttgtc cggaaagaa gtgtgtgaag aaggccttcg ggttgttaag cacttttgtc cggaaagaa gtgtgtgaag aaggccttcg ggttgtaaag cacttttgtc cggaaagaaa gtgtgtgaag aaggccttcg ggttgtaaag cacttttgtc cggaaagaaa gtgtgtgaag aaggccttcg ggttgtaaag cacttttgtc cggaaagaaa gtgtgtgaag aaggccttcg ggttgtaaag cacttttgtc cggaaagaaa gtgtgtgaag aaggccttcg ggttgtaaag cactttgtc cggaaagaa gtgtgtgaag aaggccttcg ggttgtaaag cacttttgtc cgggaagaaa gtgtgtgaag aaggccttcg ggttgtaaag cacttttgtc cggaaagaaa gtgtgtgaag aaggccttcg ggttgtaaag cacttttgtc cggaaagaa gtgtgtgaag aaggccttcg ggttgtaaag cacttttgtc cggaaagaaa gtgtgtgaag aaggccttcg ggttgtaaag cactttgtc cggaaagaaa gtgtgtgaag aaggccttcg ggttgtaaag cacttttgtc cggaaagaaa gtgtgtgaag aaggccttcg ggttgtaaag cacttttgtc cggaaagaaa gtgtgtgaag aaggccttcg ggttgtaaag cacttttgtc cggaaagaaa gtgtgtgaag aaggccttcg ggttgtaaag cacttttgtc cggaaagaaa gtgtgtgaag aaggccttcg ggttgtaaag cacttttgtc cggaaagaaa gtgtgtgaag aaggccttcg ggttgtaaag caattttgtc cggaaagaaa gtgtgtgaag aaggccttcg ggttgtaaag cacttttgtc cggaaagaaa gtgtgtgaag aaggccttcg ggttgtaaag cacttttgtc cggaaagaaa gtgtgtgaag aaggccttcg ggttgtaaag cacttttgtc aggaacgaaa gtgtgtgaag aaggccttcg ggttgtaaag cacttttgtc cggaaagaaa gtgtgtgaag aaggccttcg ggttgtaaag cacttttgtc cggaaagaaa gtgtgtgaag aaggccttcg ggttgtaaag cacttttgtc cggaaagaaa gtgagtgatg aaggccttag ggttgtaaag ctctttcgcc ggtgaagata gtgtgtgaag aaggtcttcg gattgtaaag cactttaagt tgggaggaag gtgtgtgaag aaggccttcg ggttgtaaag cactttcagc ggggaggaag

$$
410
$$

430

440

450

aggcgagggc taatatcctt tgctgatgac ggtaccggaa gaataagcac acctcgtggt taatacccgt gggggatgac ggtaccggaa gaataagcac accgcttctc taatacaggg gcgggatgac ggtaccggaa gaataagcac tcctgcctga taataccggg cggggatgac ggtaccggaa gaataagcac tccttggctc taatacagtc gggggatgac ggtaccggaa gaataagcac tcctgagggc taatatcctt cggggatgac ggtaccggaa gaataagcac 
B. cocovenenas (LMG11626) T

$B$. fungorum (LMG16225)

B. gladioli (ATCC10248) T

B. glathei (LMG14190)T

B. glathei (ATCC29195) T

B. glathei (ATCC29195)T

B. glumae (LMG2196) T

B. graminis (C4D1M) T

B. hospita (LMG20598) T

B. mallei (ATCC23344)T

B. multivorans (LMG13010) T

B. norimbergensis (Y09879) T

B. phenazinium (LMG2247) T

B. phenoliruptrix (AC1100) T

B. phymatum (STM815) T

B. phytofirmans (PsJN) T

B. pickettii (ATCC27511)T

B. pseudomallei (ATCC23343) T

B. pyrrocinia (LMG14191) T

B. pyrrocinia ATCC15958)T

B. sacchari (AF263278)T

B. silvatlantica (SRMrh-20) T

B. solanacearum (ATCC11696) T

B. sordicola (S5-B) T

B. stabilis (LMG14294) T

B. terrae (KMY02) T

B. terriccola (LMG20594) T

B. thailandensis (E264) T

B. tropicalis (Ppe8) T

B. tuberum (STM678) T

B. unamae (MT1-641) T

B. vandii (LMG16020) T

B. vietnamiensis (AMMD) $T$

B. vietnamiensis (TVV75) T

B. vietnamiensis (TVV70) T

B. plantarii (LMG9035) T

B. xenovorans (LB400) T

B. cenocepacia (KN9) T

B. ferrariae (FeG101) T

B. ambifaria (LMG19182) T

B. oklahomensis (C6786) T

B. ubonensis (GTC-P3-415) T

WC5.4C $L$. ambigua SA

WC5.4d $L$. ambigua SA

WC5.4e L. ambigua SA

WC21.1i $L$. pauciflora SA

WC21.1b L. pauciflora SA

WC21.1j L. pauciflora SA

WC26.1d L. meyerianna SA

WC26.1e L. meyerianna SA

WC26.1f $L$. meyerianna SA

P. lemoignei (LMG2207)

$R$. detusculanense (APF11)

$R$. solanacearum (R221)

R. pickettii (ATCC27512)

Az. Caulinodans (LMG6465)

$P$. aeruginosa (ATCC10145)

$K$. pneumoniae (AU45)

B. andropogonis (ATCC23061) T

$B$. fungorum (LMG19076) T

B. caribiensi (MWAP64) T

B. caryophylii (ATCC25418) T

B. cepacia (ATCC25416) T

B. cocovenenas (ATCC33664) T

B. cocovenenas (LMG11626) T

$B$. fungorum (LMG16225)

B. gladioli (ATCC10248)T

B. glathei (LMG14190)T

B. glathei (ATCC29195) T

B. glathei (ATCC29195) T

B. glumae (LMG2196) T

B. graminis (C4D1M) T

B. hospita (LMG20598) T

B. mallei (ATCC23344)T

B. multivorans (LMG13010) T tcctgagggc taatatcctt cggggatgac ggtaccggaa gaataagcac acgccgtggt taatacccgt ggcggatgac ggtaccggaa gaataagcac tcctgagggc taatatcctt cggggatgac ggtaccggaa gaataagcac acttcggggc taatacctct ggaggatgac ggtaccggaa gaataagcac acttcggggc taatacctct ggaggatgac ggtaccggaa gaataagcac acttcggggc taatacctct ggaggatgac ggtaccggaa gaataagcac tcctgagggc taatatcctt cggggatgac ggtaccggaa gaataagcac actgcctggc taatatccgg gcgggatgac ggtaccggaa gaataagcac accgcytskc taatacaggg gkgggatgac ggtaccggaa gaataagcac tcattctggc taatacccgg agtggatgac ggtaccggaa gaataagcac tccttggctc taatacagtc gggggatgac ggtaccggaa gaataagcac tcctttgggt taatacctcg gggggatgac ggtaccggaa gaataagcac tcctctgggt taataccccg gggggatgac ggtaccggaa gaataagcac acgtcgtggc taatacccgt ggcggatgac ggtaccggaa gaataagcac acttcgccgc caataccggt ggaggatgac ggtaccggaa gaataagcac acctcttggt taatacctga gggggatgac ggtaccggaa gaataagcac tggctctggt taatacctgg ggtcgatgac ggtaccggaa gaataaggac tcattctggc taatacccgg agtggatgac ggtaccggaa gaataagcac tccttggttc taatataacc gggggatgac ggtaccggaa gaataagcac tccttggttc taatatagcc gggggatgac ggtaccggaa gaataagcac tcctttgggc taataccccg gggggatgac ggtaccggaa gaataagcac tccttttcga taataccgga aggggatgac ggtaccggaa gaataagcac tcgcttcggt taatacctgg agtggatgac ggtaccggaa gaataaggac accttcgctc taatacagtg gagggatgac ggtaccggaa gaataagcac tccttggttc taatatagc gggggatgac ggtaccggaa gaataagcac acctccgtcc taatacggtg gggggatgac ggtaccggaa gaataagcac acctcagccc taatacggcg gggggatgac ggtaccggaa gaataagcac tcatcctggc taataaccgg ggtggatgac ggtaccggaa gaataagcac tccctggtcc taatatggec gggggatgac ggtaccggaa gaataagcac acctcgtggc taatatccgt gagggatgac ggtaccggaa gaataagcac tcctttgggc taatacccta gggggatgac ggtaccggaa gaataagcac tcctgagggc taatatcctt cggggatgac ggtaccggaa gaataagcac tccttggttc taatatagcc gggggatgac ggtaccggaa gaataagcatccttggctc taatacagtc gggggatgac ggtaccggaa gaataagcac tccttggctc taatacagtc gggggatgac ggtaccggaa gaataagcac tcctgaggge taatatcctt cggggatgac ggtaccggaa gaataagcac acctctgccc taatacggtg gggggatgac ggtaccggaa gaataagcac tccttggctc taatacagtc gggggatgac ggtaccggaa gaataagcac tcccctgccc taatacggcg gggggatgac ggtaccggaa gaataagcac tccttggttc taatatagcc gggggatgac ggtaccggaa gaataagcac tcctaccgac taatactggg tggggatgac ggtaccggaa gaataagcac tccttggecc taatatggec gggggatgac ggtaccggaa gaataagcac acctcgtggc taatatccgt gagggatgac ggtaccggaa gaataagcac actcccgggt taataccctg gggggatgac ggtaccggaa gaataagcac actcccgggt taataccctg gggggatgac ggtaccggaa gaataagcac gctccggggc taatacccct gggggatgac ggtaccggaa gaataagcac gctccggggc taatacccct gggggatgac ggtaccggaa gaataagcac gcattcctgt taataccggg ggkggatgac ggtaccggaa gaataagcac gcattcctgt taataccggg ggtggatgac ggtaccggaa gaataagcac gcattcctgt taataccggg ggtggatgac ggtaccggaa gaataagcac gcattcctgt taataccggg ggtggatgac ggtaccggaa gaataagcac cggagctggc taatacctgg ctctaatgac ggtacctgaa gaataagcac tggctctggt taatacctgg ggtcgatgac ggtaccggaa gaataaggac tcgcttcggt taatacctgg agtggatgac ggtaccggaa gaataaggac tggctctggt taatacctgg ggtcgatgac ggtaccggaa gaataaggac ggcagtaagt taataccttg ctgttttgac gttaccaaca gaataagcac gcggtgaggt taatagcctc atcgattgac gttacccgca gaagaagcac

460 470

480

490

500

cggctaacta cgtgccagca - gccgcgg-t aatacgta-g ggtgcgagcg cggctaacta cgtgccagca -gccgcgg-t aatacgta-g ggtgcaagcg cggctaacta cgtgccagca -gccgcgg-t aatacgta-g ggtgcaagcg cggctaacta cgtgccagca - gccgcgg-t aatacgta-g ggtgcgagcg cggctaacta cgtgccagca -gccgcgg-t aatacgta-g ggtgcaagcg cggctaacta cgtgccagca -gccgcgg-t aatacgta-g ggtgcgagcg cggctaacta cgtgccagca -gccgcgg-t aatacgta-g ggtgcgagcg cggctaacta cgtgccagca -gccgcgg-t aatacgta-g ggtgcaagcg cggctaacta cgtgccagca - gccgcgg-t aatacgta-g ggtgcgagcg cggctaacta cgtgccagca -gccgcgg-t aatacgta-g ggtgcgagcg cggctaacta cgtgccagca -gccgcgg-t aatacgta-g ggtgcgagcg cggctaacta cgtgccagca - gccgcgg-t aatacgta-g ggtgcgagcg cggctaacta cgtgccagca -gccgcgg-t aatacgta-g ggtgcgagcg cggctaacta cgtgccagca -gccgcgg-t aatacgta-g ggtgcaagcg cggctaacta cgtgccagca -gccgcgg-t aatacgta-g ggtgcgagcg cggctaacta cgtgccagca -gccgcgg-t aatacgta-g ggtgcgagcg cggctaacta cgtgccagca -gccgcgg-t aatacgta-g ggtgcgagcg 
B. norimbergensis (Y09879) T

B. phenazinium (LMG2247) T

B. phenoliruptrix (AC1100) T

B. phymatum (STM815) T

B. phytofirmans (PsJN) T

B. pickettii (ATCC27511)T

B. pseudomallei (ATCC23343) T

B. pyrrocinia (LMG14191) T

B. pyrrocinia ATCC15958) T

B. sacchari (AF263278)T

B. silvatlantica (SRMrh-20) T

B. solanacearum (ATCC11696) T

B. sordicola (S5-B) T

B. stabilis (LMG14294) T

B. terrae (KMY02) T

B. terriccola (LMG20594) T

B. thailandensis (E264)T

B. tropicalis (Ppe8) T

B. tuberum (STM678) T

B. unamae (MT1-641) T

B. vandi $($ LMG16020)T

B. vietnamiensis (AMMD) $T$

B. vietnamiensis (TVV75) T

B. vietnamiensis (TVV70) T

B. plantarii (LMG9035) T

B. xenovorans (LB400) T

B. cenocepacia (KN9) T

B. ferrariae (FeG101) T

B. ambifaria (LMG19182) T

B. oklahomensis (C6786) T

$B$. ubonensis (GTC-P3-415) T

WC5.4C L. ambigua SA

WC5.4d $L$. ambigua SA

WC5.4e $L$. ambigua SA

WC21.1i $L$. pauciflora SA

WC21.1b L. pauciflora SA

WC21.1j $L$. pauciflora SA

WC26.1d L. meyerianna SA

WC26.1e L. meyerianna SA

WC26.1f L. meyerianna SA

$P$. lemoignei (LMG2207)

$R$. detusculanense (APF11)

$R$. solanacearum (R221)

R. pickettii (ATCC27512)

Az. caulinodans (LMG6465)

$P$. aeruginosa (ATCC10145)

$K$. pneumoniae (AU45)

B. andropogonis (ATCC23061) T

$B$. fungorum (LMG19076) T

B. caribiensi (MWAP64) T

B. caryophylii (ATCC25418) T

B. cepacia (ATCC25416) T

B. cocovenenas (ATCC33664) T

B. cocovenenas (LMG11626) T

$B$. fungorum (LMG16225)

B. gladioli (ATCC10248) T

B. glathei (LMG14190)T

B. glathei (ATCC29195) T

B. glathei (ATCC29195)T

B. glumae (LMG2196) T

B. graminis (C4D1M) T

B. hospita (LMG20598) T

B. mallei (ATCC23344)T

B. multivorans (LMG13010) T

$B$. norimbergensis (Y09879) T

B. phenazinium (LMG2247) T

B. phenoliruptrix (AC1100) T

B. phymatum (STM815) T

B. phytofirmans (PsJN) T

B. pickettii (ATCC27511)T

B. pseudomallei (ATCC23343) T

B. pyrrocinia (LMG14191) T

B. pyrrocinia ATCC15958) T

B. sacchari (AF263278) T

B. silvatlantica (SRMrh-20) T cggctaacta cgtgccagca -gccgcgg-t aatacgta-g ggtgcaagcg cggctaacta cgtgccagca -gccgcgg-t aatacgta-g ggtgcaagcg cggctaacta cgtgccagca - gccgcgg-t aatacgta-g ggtgcaagcg cggctaacta cotgccagca - gccgcgg-t aatacgta-g ggtgcgagco cggctaacta cgtgccagca -gccgcgg-t aatacgta-g ggtgcaagcg cggctaacta cgtgccagca -gccgcgg-t aatacgta-g ggtccaagcg cggctaacta cgtgccagca -gccgcgg-t aatacgta-g ggtgcgagcg cggctaacta cgtgccagca -gccgcgg-t aatacgta-g ggtgcgagcg cggctaacta cgtgccagca - gccgcgg-t aatacgta-g ggtgcgagcg cggctaacta cgtgccagca -gccgcgg-t aatacgta-g ggtgcaagcg cggctaacta cgtgccagca -gccgcgg-t aatacgta-g ggtgcgagcg cggctaacta cgtgccagca -gccgcgg-t aatacgta-g ggtccaagco cggctaacta cgtgccagca -gccgcgg-t aatacgta-g ggtgcgagcg cggctaacta cgtgccagca -gccgcgg-t aatacgta-g ggtgcgagcg cggctaacta cotgccagca - gccgcgg-t aatacgta-g ggtgcaagcg cggctaacta cgtgccagca -gccgcgg-t aatacgta-g ggtgcaagcg cggctaacta cgtgccagca -gccgcgg-t aatacgta-g ggtgcgagcg cggctaacta cgtgccagca -gccgcgg-t aatacgta-g ggtgcaagcg cggctaacta cgtgccagca - gccgcgg-t aatacgta-g ggtgcgagcg cggctaacta cgtgccagca -gccgcgg-t aatacgta-g ggtgcaagcg cggctaacta cgtgccagca -gccgcgg-t aatacgta-g ggtgcgagcg cggctaacta cgtgccagca -gccgcgg-t aatacgta-g ggtgcaagcg cggctaacta cgtgccagca -gccgcgg-t aatacgta-g ggtgcaagco cggctaacta cgtgccagca -gccgcgg-t aatacgta-g ggtgcaagcg cggctaacta cgtgccagca - gccgcgg-t aatacgta-g ggtgcgagcg cggctaacta cgtgccagca -gccgcgg-t aatacgta-g ggtgcaagco cggctaacta cgtgccagca -gccgcgg-t aatacgta-g ggtgcgagcg cggctaacta cgtgccagca -gccgcgg-t aatacgta-g ggtgcgagco cggctaacta cgtgccagca - gccgcgg-t aatacgta-g ggtgcgagco cggctaacta cgtgccagca -gccgcgg-t aatacgta-g ggtgcgagcg cggctaacta cgtgccagca -gccgcgg-t aatacgta-g ggtgcgagcg cggctaacta cgtgccagca -gccgcgg-t aatacgta-g ggtgcgagcg cggctaacta cgtgccagca -gccgcgg-t aatacgta-g ggtgcaagcg cggctaacta cgtgccagca -gccgcgg-t aatacgta-g ggtgcaagcg cggctaacta cgtgccagca -gccgcgg-t aatacgta-g ggtgcgagcg cggctaacta cgtgccagca - gccgcgg-t aatacgta-g ggtgcgagcg cggctaacta cgtgccagca -gccgcgg-t aatacgta-g ggtgcgagcg cggctaacta cgtgccagca -gccgcgg-t aatacgta-g ggtgcgagcg cggctaacta cgtgccagca -gccgcgg-t aatacgta-g ggtgcgagcg cggctaacta cgtgccagca ggccgcggtt aatacgtagg ggtgcgagcg cggctaacta cgtgccagca -gccgcgg-t aatacgta-g ggtgcgagcg cggctaacta cgtgccagca - gccgcgg-t aatacgta-g ggtccaagcg cggctaacta cgtgccagca -gccgcgg-t aatacgta-g ggtccaagcg cggctaacta cgtgccagca -gccgcgg-t aatacgta-g ggtccaagcg cggctaactt cgtgccagca - gccgcgg-t aatacgaa-g ggggcaagcg cggctaactt cgtgccagca -gccgcgg-t aatacgaa-g ggtgcaagcg cggctaactc cgtgccagca -gccgcgg-t aatacgga-g ggtgcaagcg$$
510
$$

520

530

540

550

ttaatcggaa ttactgggcg taaagcgtgc gcaggcggtt tgttaagacc ttaatcggaa ttactgggcg taaagcgtgc gcaggcggtt cgctaagaca ttaatcggaa ttactgggcg taaagcgtgc gcaggcggtt cgctaagacc ttaatcggaa ttactgggcg taaagcgtgc gcaggcggtt cgctaagacc ttaatcggaa ttactgggcg taaagcgtgc gcaggcggtt tgctaagacc ttaatcggaa ttactgggcg taaagcgtgc gcaggcggtt tgttaagacc ttaatcggaa ttactgggcg taaagcgtgc gcaggcggtt tgttaagacc ttaatcggaa ttactgggcg taaagcgtgc gcaggcggtc cgctaagaca ttaatcggaa ttactgggcg taaagcgtgc gcaggcggtt tgttaagacc ttaatcggaa ttactgggcg taaagcgtgc gcaggcggtc tgttaagaca ttaatcggaa ttactgggcg taaagcgtgc gcaggcggtc tgttaagaca ttaatcggaa ttactgggcg taaagcgtgc gcaggcggtc tgttaagaca ttaatcggaa ttactgggcg taaagcgtgc gcaggcggtt tgctaagacc ttaatcggaa ttactgggcg taaagcgtgc gcaggcggtt cgctaagaca ttaatcggaa ttactgggcg taaagcgtgc gcaggcggtt cgctaagacc ttaatcggaa ttactgggcg taaagcgtgc gcaggcggtt tgctaagacc ttaatcggaa ttactgggcg taaagcgtgc gcaggcggtc tgttaagaca ttaatcggaa ttactgggcg taaagcgtgc gcaggcggtt ttgtaagacg ttaatcggaa ttactgggcg taaagcgtgc gcaggcggtc cgctaagaca ttaatcggaa ttactgggcg taaagcgtgc gcaggcggtt cgctaagaca ttaatcggaa ttactgggcg taaagcgtgc gcaggcggtt cgctaagacc ttaatcggaa ttactgggcg taaagcgtgc gcaggcggtt cgctaagaca ttaatcggaa ttactgggcg taaagcgtgc gcaggcggtt gtgcaagacc ttaatcggaa ttactgggcg taaagcgtgc gcaggcggtt tgctaagacc ttaatcggaa ttactgggcg taaagcgtgc gcaggcggtt tgctaagacc ttaatcggaa ttactgggcg taaagcgtgc gcaggcggtt tgctaagacc ttaatcggaa ttactgggcg taaagcgtgc gcaggcggtg atgcaagacc ttaatcggaa ttactgggcg taaagcgtgc gcaggcggtg atgtaagacc 
B. solanacearum (ATCC11696) T

B. sordicola (S5-B) T

B. stabilis (LMG14294)T

$B$. terrae (KMY02) T

B. terriccola (LMG20594) T

$B$. thailandensis (E264) T

B. tropicalis (Ppe8) T

B. tuberum (STM678) T

B. unamae (MT1-641) T

B. vandii (LMG16020) T

$B$. vietnamiensis (AMMD) T

B. vietnamiensis (TVV75) T

B. vietnamiensis (TVV70)T

B. plantarii (LMG9035) T

B. xenovorans (LB400) T

B. cenocepacia (KN9) T

$B$. ferrariae (FeG101) T

B. ambifaria (LMG19182) T

B. oklahomensis (C6786) T

B. ubonensis (GTC-P3-415) T

WC5.4C $L$. ambigua SA

WC5.4d $L$. ambigua SA

WC5.4e $L$. ambigua SA

WC21.1i L. pauciflora SA

WC21.1b $L$. pauciflora SA

WC21.1j $L$. pauciflora SA

WC26.1d $L$. meyerianna SA

WC26.1e $L$. meyerianna SA

WC26.1f $L$. meyerianna SA

$P$. lemoignei (LMG2207)

$R$. detusculanense (APF11)

$R$. solanacearum (R221)

R. pickettii (ATCC27512)

Az. caulinodans (LMG6465)

$P$. aeruginosa (ATCC10145)

$K$. pneumoniae (AU45)

B. andropogonis (ATCC23061) T

$B$. fungorum (LMG19076) T

B. caribiensi (MWAP64) T

B. caryophylii (ATCC25418)T

B. cepacia (ATCC25416) T

B. cocovenenas (ATCC33664)T

B. cocovenenas (LMG11626) T

$B$. fungorum (LMG16225)

B. gladioli (ATCC10248) T

B. glathei (LMG14190) T

B. glathei (ATCC29195)T

B. glathei (ATCC29195) T

B. glumae (LMG2196) T

B. graminis (C4D1M) T

B. hospita (LMG20598) T

B. mallei (ATCC23344) T

B. multivorans (LMG13010) T

$B$. norimbergensis (Y09879) T

B. phenazinium (LMG2247) T

B. phenoliruptrix (AC1100) T

B. phymatum (STM815) T

B. phytofirmans (PsJN) T

B. pickettii (ATCC27511) T

B. pseudomallei (ATCC23343) T

B. pyrrocinia (LMG14191) T

B. pyrrocinia ATCC15958) T

B. sacchari (AF263278) T

$B$. silvatlantica (SRMrh-20)T

B. solanacearum (ATCC11696) T

B. sordicola (S5-B) T

B. stabilis (LMG14294) T

B. terrae (KMY02) T

B. terriccola (LMG20594) T

B. thailandensis (E264) T

B. tropicalis (Ppe8) T

B. tuberum (STM678) T

B. unamae (MT1-641) T

B. vandii (LMG16020) T

$B$. vietnamiensis (AMMD) T ttaatcggaa ttactgggcg taaagcgtgc gcaggcggtt gtgcaagacc ttaatcggaa ttactgggcg taaagcgtgc gcaggcggtt cgttaagaca ttaatcggaa ttactgggcg taaagcgtgc gcaggcggtt tgctaaracc ttaatcggaa ttactgggcg taaagcgtgc gcaggcggtt cgctaagacc ttaatcggaa ttactgggcg taaagcgtgc gcaggcggtc cgctaagaca ttaatcggaa ttactgggcg taaagcgtgc gcaggcggtt tgctaagacc ttaatcggaa ttactgggcg taaagcgtgc gcaggcggtg atgtaagacc ttaatcggaa ttactgggcg taaagcgtgc gcaggcggtg ctgtaagacc ttaatcggaa ttactgggcg taaagcgtgc gcaggcggtg atgcaagacc ttaatcggaa ttactgggcg taaagcgtgc gcaggcggtt tgttaagacc ttaatcggaa ttactgggcg taaagcgtgc gcaggcggtt tgttaag--c ttaatcggaa ttactgggcg taaagcgtgc gcaggcggtt tgctaagacc ttaatcggaa ttactgggcg taaagcgtgc gcaggcggtt tgctaagacc ttaatcggaa ttactgggcg taaagcgtgc gcaggcggtt tgttaagacc ttaatcggaa ttactgggcg taaagcgtgc gcaggcggtt cgctaagaca ttaatcggaa ttactgggcg taaagcgtgc gcaggcggtt tgctaagacc ttaatcggaa ttactgggcg taaagcgtgc gcaggcggtg atgtaagacc ttaatcggaa ttactgggcg taaagcgtgc gcaggcggtt tgctaagacc ttaatcggaa ttactgggcg taaagcgtgc gcaggcggtt tgttaagacc ttaatcggaa ttactgggeg taaagcgtgc gcaggcggtt tgctaagacc ttaatcggaa ttactgggcg taaagcgtgc gcaggcggtg ctgtaagacc ttaatcggaa ttactgggcg taaagcgtgc gcaggcggtt cgctaagaca ttaatcggaa ttactgggcg taaagcgtgc gcaggcggtt cgctaagaca ttaatcggaa ttactgggcg taaagcgtgc gcaggcggtg ctgtaagacc ttaatcggaa ttactgggcg taaagcgtgc gcaggcggtg ctgtaagacc ttaatcggaa ttactgggcg taaagcgtgc gcaggcggtg ctgtaagacc ttaatcggaa ttactgggcg taaagcgtgc gcaggcggtg ctgtaagacc ttaatcggaa ttactgggcg taaagcgtgc gcaggcggtg ctgtaagacc ttaatcggaa ttactgggcg taaagcgtgc gcaggcggtg ctgtaagacc ttaatcggaa ttactgggcg taaagcgtgc gcaggcggtt gtgcaagaca ttaatcggaa ttactgggcg taaagcgtgc gcaggcggtt gtgcaagacc ttaatcggaa ttactgggcg taaagcgtgc gcaggcggtt gtgcaagacc ttaatcggaa ttactgggcg taaagcgtgc gcaggcggtt gtgcaagacc ttgctcggaa tcactgggcg taaagcgcac gtaggcggat cgttaagtca ttaatcggaa ttactgggcg taaagcgcgc gtaggtggtt cagcaagttg ttaatcggaa ttactgggcg taaagcgcac gcaggcggtc tgtcaagtcg

$$
560
$$

580

590

600

gatgtgaaat ccccgggctt aacctgggaa ctgcatttgt gactggcago gatgtgaaat ccccgggctt aacctgggaa ctgcatttgt gactggcggg gatgtgaaat ccccgggctt aacctgggaa ctgcattggt gactggcggg gatgtgaaat ccccgggctt aacctgggaa ctgcattggt gactggcogg gatgtgaaat ccccgggctc aacctgggaa ctgcattggt gactggcagg gatgtgaaat ccccgggctc aacctgggaa ctgcattggt gactggcaag gatgtgaaat ccccgggctc aacctgggaa ctgcattggt gactggcaag gatgtgaaat ccccgggctt aacctgggaa ctgcatttgt gactggcggg gatgtgaaat ccccgggctc aacctgggaa ctgcattggt gactggcaag gatgtgaaat ccccgggctt aacctgggaa ctgcatttgt gactggcagg gatgtgaaat ccccgggctt aactgggaa ctgcatttgt gactggcagg gatgtgaaag ccccgggctt aacctgggaa ctgcatttgt gactggcagg gatgtgaaat ccccgggctc aactgggaa ctgcattggt gactggcagg gatgtgaaat ccccgggctt aacctgggaa ctgcatttgt gactggcggg gatgtgaaat ccccgggctt aacctgggaa ctgcattggt gactggcogg gatgtgaaat ccccgggctc aacctgggaa ctgcattggt gactggcagg gatgtgaaat ccccgggctc aacctgggaa ctgcatttgt gactggcagg gatgtgaaat ccccgggctt aacctgggaa ctgcattcgt gactgcaagg gatgtgaaat ccccgggctt aacctgggaa ctgcatttgt gactggcggg gatgtgaaat ccccgggctt aacctgggaa ctgcatttgt gactggcggg gatgtgaaat ccccgggctt aacctgggaa ctgcattggt gactggcggg gatgtgaaat ccccgggctt aacctgggaa ctgcatttgt gactggcggg gatgtgaaat ccccgagctt aacttgggaa ttgcattggt gactgcacgg gatgtgaaat ccccgggctc aactgggaa ctgcattggt gactggcagg gatgtgaaat ccccgggctc aacctgggaa ctgcattggt gactggcagg gatgtgaaat ccccgggctc aacctgggaa ctgcattggt gactggcago gatgtgaaat ccccgggctt aacctgggaa ctgcattggt gactgcatcg gatgtgaaat ccccgggctt aacctgggaa ctgcattggt gactgcatcg gatgtgaaat ccccgggctt aacctgggaa ttgcattggt gactgcacgg gatgtgaaat ccccgggctt aacctgggaa ctgcatttgt gactggcgag gatgtgaaat ccccgggctc aacctgggaa ctgcattggt gactggcagg gatgtgaaat ccccgggctt aacctgggaa ctgcattggt gactggcggg gatgtgaaat ccccgggctt aacctgggaa ctgcatttgt gactggcggg gatgtgaaat ccccgggctc aacctgggaa ctgcattggt gactggcagg gatgtgaaat ccccgggctc aacctgggaa ctgcattggt gactgcatcg gatgtgaaat ccccgggctt aacctgggaa ctgcattggt gactgcagcg gatgtgaaat ccccgggctc aacctgggaa ctgcattggt gactgcatcg gatgtgaaat ccccgggctc aacctgggaa ctgcattggt gactggcaag gatgtgaaat ccccgggctc aacctgggaa ctgcattggt gactggcaag 
B. vietnamiensis (TVV75) T

$B$. vietnamiensis (TVV70) T

B. plantarii (LMG9035) T

B. xenovorans (LB400) T

$B$. cenocepacia (KN9) T

B. ferrariae (FeG101) T

B. ambifaria (LMG19182) T

$B$. oklahomensis (C6786) T

B. ubonensis (GTC-P3-415) T

WC5.4C $L$. ambigua SA

WC5.4d $L$. ambigua SA

WC5.4e $L$. ambigua SA

WC21.1i L. pauciflora SA

WC21.1b $L$. pauciflora SA

WC21.1j $L$. pauciflora SA

WC26.1d $L$. meyerianna SA

WC26.1e $L$. meyerianna SA

WC26.1f $L$. meyerianna SA

$P$. lemoignei (LMG2207)

$R$. detusculanense (APF11)

$R$. solanacearum (R221)

R. pickettii (ATCC27512)

Az. caulinodans (LMG6465)

$P$. aeruginosa (ATCC10145)

$K$. pneumoniae (AU45)

B. andropogonis (ATCC23061) T

$B$. fungorum (LMG19076) $T$

B. caribiensi (MWAP64) T

B. caryophylii (ATCC25418)T

B. cepacia (ATCC25416) T

B. cocovenenas (ATCC33664)T

$B$. cocovenenas (LMG11626) T

$B$. fungorum (LMG16225)

B. gladioli (ATCC10248) T

B. glathei (LMG14190) T

B. glathei (ATCC29195) T

B. glathei (ATCC29195) T

B. glumae (LMG2196) T

$B$. graminis (C4D $1 \mathrm{M}) \mathrm{T}$

B. hospita (LMG20598) T

B. mallei (ATCC23344) T

B. multivorans (LMG13010) T

B. norimbergensis (Y09879) T

B. phenazinium (LMG2247)T

B. phenoliruptrix (AC1100) T

B. phymatum (STM815) T

B. phytofirmans (PsJN) T

B. pickettii (ATCC27511)T

B. pseudomallei (ATCC23343) T

B. pyrrocinia (LMG14191) T

B. pyrrocinia ATCC15958)T

B. sacchari (AF263278) T

B. silvatlantica (SRMrh-20)T

B. solanacearum (ATCC11696) T

$B$. sordicola (S5-B) $T$

B. stabilis (IMG14294) T

B. terrae (KMY02) T

B. terriccola (LMG20594) T

B. thailandensis (E264) T

$B$. tropicalis (Ppe8) T

B. tuberum (STM678) T

B. unamae (MT1-641) T

$B$. vandii (LMG16020) T

$B$. vietnamiensis (AMMD) T

B. vietnamiensis (TVV75) T

B. vietnamiensis (TVV70)T

B. plantarii (LMG9035) T

B. xenovorans (LB400) T

$B$. cenocepacia (KN9) T

B. ferrariae (FeG101) T

B. ambifaria (LMG19182) T

B. oklahomensis (C6786) T

B. ubonensis (GTC-P3-415) T

WC5.4C $L$. ambigua SA

WC5.4d $L$. ambigua SA gatgtgaaat ccccgggctc aacctgggaa ctgcattggt gactggcagg gatgtgaaat ccccgggctc aacctgggaa ctgcattggt gactggcagg gatgtgaaat ccccgggctc aacctgggaa ctgcattggt gactggcaag gatgtgaaat ccccgggctt aacctgggaa ctgcatttgt gactggcggg gatgtgaaat ccccgggctc aactgggaa ctgcattggt gactggcagg gatgtgaaat ccccgggctc aacctgggaa ctgcattggt gactgcattg gatgtgaaat ccccgggctc aacctgggaa ctgcattggt gactggcagg gatgtgaaat ccccgggctc aacctgggaa ctgcattggt gactggcaag gatgtgaaat ccccgggctc aacctgggaa ctgcattggt gactggcagg gatgtgaaat ccccgggctt aacctgggaa ctgcattggt gactgcagcg gatgtgaaat ccccgggctt aactgggaa ctgcatttgt gactggcggg gatgtgaaat ccccgggctt aacctgggaa ctgcatttgt gactggcggo gatgtgaaat ccccgggctt aacctgggaa ctgcattggt gactgcagcg gatgtgaaat ccccgggctt aacctgggaa ctgcattggt gactgcagcg gatgtgaaat ccccgggctt aacctgggaa ctgcattggt gactgcagcg gatgtgaaat ccccgggctt aacctgggaa ctgcattggt gactgcagcg gatgtgaaat ccccgggctt aacctgggaa ctgcattggt gactgcagcg gatgtgaaat ccccgggctt aacctgggaa ctgcattggt gactgcagco gaggtgaaat ccccgagctt aacttgggaa ctgcctttgt gactgcatgg gatgtgaaat ccccgagctt aacttgggaa ttgcattggt gactgcacgg gatgtgaaat ccccgggctt aacctgggaa ttgcattggt gactgcacgg gatgtgaaat ccccgagctt aacttgggaa ttgcattggt gactgcacgg ggggtgaaag cctggagctc aactccagaa ctgccettga tactggcgat gatgtgaaat ccccgggctc aacctgggaa ctgcatccaa actactgag gatgtgaaat ccccgggctc aacctgggaa ctgcattcga aactggcagg

610 620 630 640 650 ctagagtgtg gcaga-ggg- gggtagaatt -ccgcgtgta -gcagtgaaa ctagagtatg gcaga-ggg- gggtagaatt -ccacgtgta -gcagtgaaa ctagagtatg gcaga-ggg- gggtagaatt -ccacgtgta -gcagtgaaa ctagagtatg gcaga-ggg- gggtagaatt -ccacgtgta -gcagtgaaa ctagagtatg gcaga-ggg- gggtagaatt -ccacgtgta -gcagtgaaa ctagagtatg gcaga-ggg- gggtagaatt -ccacgtgta -gcagtgaaa ctagagtatg gcaga-ggg- gggtagaatt -ccacgtgta -gcagtgaaa ctagagtatg gcaga-ggg- gggtagaatt -ccacgtgta -gcagtgaaa ctagagtatg gcaga-ggg- gggtagaatt -ccacgtgta -gcagtgaaa ctagagtatg gcaga-ggg- gggtagaatt -ccacgtgta -gcagtgaaa ctagagtatg gcaga-ggg- gggtagaatt -ccacgtgta -gcagtgaaa ctagagtatg gcaga-ggg- gggtagaatt -ccacgtgta -gcagtgaaa ctagagtatg gcaga-ggg- gggtagaatt -ccacgtgta -gcagtgaaa ctagagtatg gcaga-ggg- gggtagaatt -ccacgtgta -gcagtgaaa ctagagtatg gcaga-ggg- gggtagaatt -ccacgtgta -gcagtgaaa ctagagtatg gcaga-ggg- gggtagaatt -ccacgtgta -gcagtgaaa ctagagtatg gcaga-ggg- gggtagaatt -ccacgtgta -gcagtgaaa ctagagtatg gcaga-ggg- gggtagaatt -ccacgtgta -gcagtgaaa ctagagtatg gcaga-ggg- gggtagaatt -ccacgtgta -gcagtgaaa ctagagtatg gcaga-ggg- gggtagaatt -ccacgtgta -gcagtgaaa ctagagtatg gcaga-ggg- gggtagaatt -ccacgtgta -gcagtgaaa ctagagtatg gcaga-ggg- gggtagaatt -ccacgtgta -gcagtgaaa ctagagtgtg tcaga-ggg- gggtagaatt -ccacgtgta -gcagtgaaa ctagagtatg gcaga-ggg- gggtagaatt -ccacgtgta -gcagtgaaa ctagagtatg gcaga-ggg- gggtagaatt -ccacgtgta -gcagtgaaa ctagagtatg gcaga-ggg- gggtagaatt -ccacgtgta -gcagtgaaa ctggagtatg gcaga-ggg- gggtagaatt -ccacgtgta -gcagtgaaa ctggagtatg gcaga-ggg- gggtagaatt -ccacgtgta -gcagtgaaa ctagagtgtg tcaga-ggg- aggtagaatt -ccacgtgta -gcagtgaaa ctagagtatg gcaga-ggg- gggtagaatt -ccacgtgta -gcagtgaaa ctagagtatg gcaga-ggg- gggtagaatt -ccacgtgta -gcagtgaaa ctagagtatg gcaga-ggg- gggtagaatt -ccacgtgta -gcagtgaaa ctagagtatg gcaga-ggg- gggtagaatt -ccacgtgta -gcagtgaaa ctagagtatg gcaga-ggg- gggtagaatt -ccacgtgta -gcagtgaaa cttgagtatg gcaga-ggg- gggtagaatt -ccacgtgta -gcagtgaaa ctggagtatg gcaga-ggg- gggtggaatt -ccacgtgta -gcagtgaaa ctagagtatg gcaga-ggg- gggtagaatt -ccacgtgta -gcagtgaaa ctagagtatg gcaga-ggg- gggtagaatt -ccacgtgta -gcagtgaaa ctagagtatg gcag---gg- gggtag-att -ccacgtgta -gcagtgaaa ctagagtatg gcaga-ggg- gggtagaatt -ccacgtgta -gcagtgaaa ctagagtatg gcaga-ggg- gggtagaatt -ccacgtgta -gcagtgaaa ctagagtatg gcaga-ggg- gggtagaatt -ccacgtgta -gcagtgaaa ctagagtatg gcaga-ggg- gggtagaatt -ccacgtgta -gcagtgaaa ctagagtatg gcaga-ggg- gggtagaatt -ccacgtgta -gcagtgaaa ctcgagtatg gcaga-ggg- gggtggaatt -ccacgtgta -gcagtgaaa ctagagtatg gcaga-ggg- gggtagaatt -ccacgtgta ggcagtgaaa ctagagtatg gcaga-ggg- gggtagaatt -ccacgtgta -gcagtgaaa ctagagtatg gcaga-ggg- gggtagaatt -ccacgtgta -gcagtgaaa ctggagtatg gcaga-ggg- gggtggaatt -ccacgtgta -gcagtgaaa ctagagtatg gcaga-ggg- gggtagaatt -ccacgtgta -gcagtgaaa 
WC5.4e $L$. ambigua SA WC21.1i L. pauciflora SA WC21.1b $L$. pauciflora SA WC21.1j $L$. pauciflora SA WC26.1d $L$. meyerianna SA WC26.1e $L$. meyerianna SA WC26.1f $L$. meyerianna SA $P$. lemoignei (LMG2207)

$R$. detusculanense (APF11)

$R$. solanacearum (R221)

R. pickettii (ATCC27512)

Az. caulinodans (LMG6465)

$P$. aeruginosa (ATCC10145)

$K$. pneumoniae (AU45)

B. andropogonis (ATCC23061) T

$B$. fungorum (LMG19076) $T$

B. caribiensi (MWAP64) T

B. caryophylii (ATCC25418) T

B. cepacia (ATCC25416) T

B. cocovenenas (ATCC33664) T

$B$. cocovenenas (LMG11626) T

$B$. fungorum (LMG16225)

B. gladioli (ATCC10248) T

B. glathei (LMG14190) T

B. glathei (ATCC29195)T

B. glathei (ATCC29195) T

B. glumae (LMG2196) T

$B$. graminis (C4D1M) T

B. hospita (LMG20598) T

B. mallei (ATCC23344) T

B. multivorans (LMG13010) T

B. norimbergensis (Y09879) T

B. phenazinium (LMG2247)T

B. phenoliruptrix (AC1100) T

B. phymatum (STM815) T

B. phytofirmans (PsJN) T

B. pickettii (ATCC27511)T

B. pseudomallei (ATCC23343) T

B. pyrrocinia (LMG14191) T

B. pyrrocinia ATCC15958)T

B. sacchari (AF263278) T

B. silvatlantica (SRMrh-20) T

B. solanacearum (ATCC11696) T

B. sordicola (S5-B) T

B. stabilis (LMG14294) T

B. terrae (KMY02) T

B. terriccola (LMG20594) T

$B$. thailandensis (E264) T

B. tropicalis (Ppe8) T

B. tuberum (STM678) T

B. unamae (MT1-641) T

$B$. vandii (LMG16020) T

$B$. vietnamiensis (AMMD) T

B. vietnamiensis (TVV75) T

$B$. vietnamiensis (TVV70)T

B. plantarii (LMG9035) T

B. xenovorans (LB400) T

$B$. cenocepacia (KN9) T

$B$. ferrariae (FeG101) T

B. ambifaria (LMG19182) T

$B$. oklahomensis (C6786) T

B. ubonensis (GTC-P3-415) T

WC5.4C $L$. ambigua SA

WC5.4d $L$. ambigua SA

WC5.4e $L$. ambigua SA

WC21.1i L. pauciflora SA

WC21.1b $L$. pauciflora SA

WC21.1j $L$. pauciflora SA

WC26.1d $L$. meyerianna SA

WC26.1e L. meyerianna SA

WC26.1f $L$. meyerianna SA

$P$. lemoignei (LMG2207)

$R$. detusculanense (APF11)

R. solanacearum (R221)

R. pickettii (ATCC27512) ctagagtatg gcaga-ggg- gggtagaatt -ccacgtgta -gcagtgaaa ctggagtatg gcaga-ggg- gggtggaatt -ccacgtgta -gcagtgaaa ctggagtatg gcagagggg- gggtggaatt -ccacgtgta -gcagtgaaa ctggagtatg gcaga-gggc gggtggaatt -ccacgtgta -gcagtgaaa ctggagtatg gcaga-ggg- gggtggaatt -ccacgtgta -gcagtgaaa ctggagtatg gcaga-ggg- gggtggaatt cccacgtgta -gcagtgaaa ctggagtatg gcaga-ggg- gggtggaatt -ccacgtgta -gcagtgaaa ctagagtgtg tcaga-ggg- gggtagaatt -ccacgtgta -gcagtgaaa ctagagtgtg tcaga-ggg- gggtagaatt -ccacgtgta -gcagtgaaa ctagagtgtg tcaga-ggg- gggtagaatt -ccacgtgta -gcagtgaaa ctagagtgtg tcaga-ggg- gggtagaatt -ccacgtgta -gcagtgaaa cttgagttcg agaga-ggt- tggtggaact -ccgagtgta -gaggtgaaa ctagagtacg gtaga-ggg- tggtggaatt -tcctgtgta -gcggtgaaa ctagagtctt gtaga-ggg- gggtagaatt -ccaggtgta -gcggtgaaa$$
660
$$

680

690

700

tgcgtagata tgtggaggaa taccgatggc gaaggcagcc ccctgggcca tgcgtagaga tgtggaggaa taccgatggc gaaggcagcc ccctgggcca tgcgtagaga tgtggaggaa taccgatggc gaaggcagcc ccctgggcca tgcgtagaga tgtggaggaa taccgatggc gaaggcagcc ccctgggcca tgcgtagaga tgtggaggaa taccgatggc gaaggcagcc ccctgggcca tgcgtagaga tgtggaggaa taccgatggc gaaggcagcc ccctgggcca tgcgtagaga tgtggaggaa taccgatggc gaaggcagcc ccctgggcca tgcgtagaga tgtggaggaa taccgatggc gaaggcagcc ccctgggcca tgcgtagaga tgtggaggaa taccgatggc gaaggcagcc ccctgggcca tgcgtagaga tgtggaggaa taccgatggc gaaggcagcc ccctgggcca tgcgtagaga tgtggaggaa taccgatggc gaaggcagcc ccctgggcca tgcgtagaga tgtggaggaa taccgatggc gaaggcagcc ccctgggcca tgcgtagaga tgtggaggaa taccgatggc gaaggcagcc ccctgggcca tgcgtagaga tgtggaggaa taccgatggc gaaggcagcc ccctgggcca tgcgtagaga tgtggaggaa taccgatggc gaaggcagcc ccctgggcca tgcgtagaga tgtggaggaa taccgatggc gaaggcagcc ccctgggcca tgcgtagaga tgtggaggaa taccgatggc gaaggcagcc ccctgggcca tgcgtagaga tgtggaggaa taccgatggc gaaggcagcc ccctgcggca tgcgtagaga tgtggaggaa taccgatggc gaaggcagcc ccctgggcca tgcgtagaga tgtggaggaa taccgatggc gaaggcagcc ccctgggcca tgcgtagaga tgtggaggaa taccgatggc gaaggcagcc ccctgggcca tgcgtagaga tgtggaggaa taccgatggc gaaggcagcc ccctgggcca tgcgtagaga tgtggaggaa taccgatggc gaaggcagcc ccctgggata tgcgtagaga tgtggaggaa taccgatggc gaaggcagcc ccctgggcca tgcgtagaga tgtggaggaa taccgatggc gaaggcagcc ccctgggcca tgcotagaga tgtggaggaa taccgatggc gaaggcagcc ccctgggcca tgcgtagaga tgtggaggaa taccgatggc gaaggcagcc ccctgggcca tgcgtagaga tgtggaggaa taccgatggc gaaggcagcc ccctgggcca tgcgtagaga tgtggaggaa taccgatggc gaaggcagcc tcctgggata tgcgtagaga tgtggaggaa taccgatggc gaaggcagcc ccctgggcca tgcgtagaga tgtggaggaa taccgatggc gaaggcagcc ccctgggcca tgcgtagaga tgtggaggaa taccgatggc gaaggcagcc ccctgggcca tgcgtagaga tgtggaggaa taccgatggc gaaggcagcc ccctgggcca tgcgtagaga tgtggaggaa taccgatggc gaaggcagcc ccctgggcca tgcgtagaga tgtggaggaa taccgatggc gaaggcagcc ccctgggtca tgcgtagaga tgtggaggaa caccgatgge gaaggcagcc ccctgggcca tgcgtagaga tgtggaggaa taccgatggc gaaggcagcc ccctgggtca tgcgtagaga tgtggaggaa taccgatggc gaaggcagcc ccctgggcca tgcgtagaga tgtggaggaa taccgatggc gaaggcagcc ccctgggcca tgcotagaga tgtggaggaa taccgatggc gaaggcagcc ccctgggcca tgcgtagaga tgtggaggaa taccgatggc gaaggcagcc ccctgggcca tgcgtagaga tgtggaggaa taccgatggc gaaggcagcc ccctgggcca tgcgtagaga tgtggaggaa taccgatggc gaaggcagcc ccctgggcca tgcgtagaga tgtggaggaa taccgatggc gaaggcagcc ccctgggcca tgcotagaga tgtggaggaa caccgatggc gaaggcagcc ccctgggcca tgcgtagaga tgtggaggaa taccgatggc gaaggcagcc ccctgggcca tgcgtagaga tgtggaggaa taccgatggc gaaggcagcc ccctgggcca tgcgtagaga tgtggaggaa taccgatggc gaaggcagcc ccctgggcca tgcgtagaga tgtggaggaa caccgatggc gaaggcagcc ccctgggcca tgcgtagaga tgtggaggaa taccgatggc gaaggcagcc ccctgggcca tgcgtagaga tgtggaggaa taccgatggc gaaggcagcc ccctgggcca tgcgtagaga tgtggaggaa caccgatggc gaaggcagcc ccctgggcca tgcgtagaga tgtggaggaa caccgatggc gaaggcagcc ccctgggcca tgcgtagaga tgtggaggaa caccgatggc gaaggcagcc ccctgggcca tgcgtagaga tgtggaggaa caccgatggc gaaggcagcc ccctgggcca tgcgtagaga tgtggaggaa caccgatggc gaaggcagcc ccctgggcca tgcgtagaga tgtggaggaa caccgatggc gaaggcagcc ccctgggcca tgcgtagata tgtggaggaa taccgatggc gaaggcagcc ccctgggata tgcgtagaga tgtggaggaa taccgatggc gaaggcagcc ccctgggata tgcgtagaga tgtggaggaa taccgatggc gaaggcagcc ccctgggata tgcgtagaga tgtggaggaa taccgatggc gaaggcagcc ccctgggata 
Az. Caulinodans (LMG6465)

$P$. aeruginosa (ATCC10145)

K. pneumoniae (AU45)

B. andropogonis (ATCC23061) T

$B$. fungorum (LMG19076) T

B. caribiensi (MWAP64) T

B. caryophylii (ATCC25418) T

B. cepacia (ATCC25416) T

B. cocovenenas (ATCC33664)T

B. cocovenenas (LMG11626) T

$B$. fungorum (LMG16225)

B. gladioli (ATCC10248) T

B. glathei (LMG14190) T

B. glathei (ATCC29195)T

B. glathei (ATCC29195) T

B. glumae (LMG2196) T

$B$. graminis (C4D1M) T

B. hospita (LMG20598) T

B. mallei (ATCC23344) T

B. multivorans (LMG13010) T

B. norimbergensis (Y09879) T

B. phenazinium (LMG2247) T

B. phenoliruptrix (AC1100) T

B. phymatum (STM815) T

B. phytofirmans (PsJN) T

B. pickettii (ATCC27511) T

B. pseudomallei (ATCC23343) T

B. pyrrocinia (LMG14191)T

B. pyrrocinia ATCC15958) T

B. sacchari (AF263278) T

B. silvatlantica (SRMrh-20) T

B. solanacearum (ATCC11696)T

$B$. sordicola (S5-B) $\mathrm{T}$

B. stabilis (LMG14294) T

$B$. terrae (KMY02) T

B. terriccola (LMG20594) T

$B$. thailandensis (E264)T

B. tropicalis (Ppe8) T

B. tuberum (STM678) T

B. unamae (MT1-641) T

B. vandii (LMG16020) T

$B$. vietnamiensis (AMMD) T

$B$. vietnamiensis (TVV75) T

B. vietnamiensis (TVV70) T

B. plantarii (LMG9035) T

$B$. xenovorans (LB400) T

$B$. cenocepacia (KN9) T

B. ferrariae (FeG101) T

B. ambifaria (LMG19182) T

B. oklahomensis (C6786) T

B. ubonensis (GTC-P3-415) T

WC5.4C $L$. ambigua SA

WC5.4d $L$. ambigua SA

WC5. 4 e L. ambigua SA

WC21.1i $L$. pauciflora SA

WC21.1b $L$. pauciflora SA

WC21.1j $L$. pauciflora SA

WC26.1d $L$. meyerianna SA

WC26.1e $L$. meyerianna SA

WC26.1f $L$. meyerianna SA

$P$. lemoignei (LMG2207)

$R$. detusculanense (APF11)

$R$. solanacearum (R221)

R. pickettii (ATCC27512)

Az. Caulinodans (LMG6465)

$P$. aeruginosa (ATCC10145)

$K$. pneumoniae (AU45)

B. andropogonis (ATCC23061) T

B. fungorum (LMG19076) T

B. caribiensi (MWAP64) T

B. caryophylii (ATCC25418) T

B. cepacia (ATCC25416) T

B. cocovenenas (ATCC33664)T ttcgtagata ttcggaagaa caccagtggc gaaggcggcc aactggctco tgcgtagata taggaaggaa caccagtggc gaaggcgacc acctggactg tgcgtagaga tctggaggaa taccggtgge gaaggcggcc ccctggacaa

710

720

730

740

750

atactgacgc tcatgcacga aagcgtgggg agcaaacagg attagatacc atactgacgc tcatgcacga aagcgtgggg agcaaacagg attagatacc atactgacgc tcatgcacga aagcgtgggg agcaaacagg attagatacc atactgacgc tcatgcacga aagcgtgggg agcaaacagg attagatacc atactgacgc tcatgcacga aagcgtgggg agcaaacagg attagatacc atactgacgc tcatgcacga aagcgtgggg agcaaacagg attagatacc atactgacgc tcatgcacga aagcgtgggg agcaaacagg attagatacc atactgacgc tcatgcacga aagcgtgggg agcaaacagg attagatacc atactgacgc tcatgcacga aagcgtgggg agcaaacagg attagatacc atactgacgc tcatgcacga aagcgtgggg agcaaacagg attagatacc atactgacgc tcatgcacga aagcgtgggg agcaaacagg attagatacc atactgacgc tcatgcacga aagcgtgggg agcaaacagg attagatacc atactgacgc tcatgcacga aagcgtgggg agcaaacagg attagatacc atactgacgc tcatgcacga aagcgtgggg agcaaacagg attagatacc atactgacgc tcatgcacga aagcgtgggg agcaaacagg attagatacc atactgacgc tcatgcacga aagcgtgggg agcaaacagg attagatacc atactgacgc tcatgcacga aagcgtgggg agcaaacagg attagatacc atactgacgc tcatgcacga aagcgtgggg agcaaacagg attagatacc atactgacgc tcatgcacga aagcgtgggg agcaaacagg attagatacc atactgacgc tcatgcacga aagcgtgggg agcaaacagg attagatacc atactgacgc tcatgcacga aagcgtgggg agcaaacagg attagatacc atactgacgc tcatgcacga aagcgtgggg agcaaacagg attagatacc acactgacgc tcatgcacga aagcgtgggg agcaaacagg attagatacc atactgacgc tcatgcacga aagcgtgggg agcaaacagg attagatacc atactgacgc tcatgcacga aagcgtgggg agcaaacagg attagatacc atactgacgc tcatgcacga aagcgtgggg agcaaacagg attagatacc atactgacgc tcatgcacga aagcgtgggg agcaaacagg attagatacc atactgacgc tcatgcacga aagcgtgggg agcaaacagg attagatacc acactgacgc tcatgcacga aagcgtgggg agcaaacagg attagatacc atactgacgc tcatgcacga aagcgtgggg agcaaacagg attagatacc atactgacgc tcatgcacga aagcgtgggg agcaaacagg attagatacc atactgacgc tcatgcacga aagcgtgggg agcaaacagg attagatacc atactgacgc tcatgcacga aagcgtgggg agcaaacagg attagatacc atactgacgc tcatgcacga aagcgtgggg agcaaacagg attagatacc atactgacgc tcatgcacga aagcgtgggg agcaaacagg attagatacc atactgacgc tcatgcacga aagcgtgggg agcaaacagg attagatacc atactgacgc tcatgcacga aagcgtgggg agcaaacagg attagatacc atactgacgc tcatgcacga aagcgtgggg agcaaacagg attagatacc atactgacgc tcatgcacga aagcgtgggg agcaaacagg attagatacc atactgacgc tcatgcacga aagcgtgggg agcaaacagg attagatacc atactgacgc tcatgcacga aagcgtgggg agcaaacagg attagatacc atactgacgc tcatgcacga aagcgtgggg agcaaacagg attagatacc atactgacgc tcatgcacga aagcgtgggg agcaaacagg attagatacc atactgacgc tcatgcacga aagcgtgggg agcaaacagg attagatacc atactgacgc tcatgcacga aagcgtgggg agcaaacagg attagatacc atactgacgc tcatgcacga aagcgtgggg agcaaacagg attagatacc atactgacgc tcatgcacga aagcgtgggg agcaaacagg attagatacc atactgacgc tcatgcacga aagcgtgggg agcaaacagg attagatacc atactgacgc tcatgcacga aagcgtgggg agcaaacagg attagatacc atactgacgc tcatgcacga aagcgtgggg agcaaacagg attagatacc atactgacgc tcatgcacga aagcgtgggg agcaaacagg attagatacc atactgacgc tcatgcacga aagcgtgggg agcaaacagg attagatacc atactgacgc tcatgcacga aagcgtgggg agcaaacagg attagatacc atactgacgc tcatgcacga aagcgtgggg agcaaacagg attagatacc atactgacgc tcatgcacga aagcgtgggg agcaaacagg attagatacc atactgacgc tcatgcacga aagcgtgggg agcaaacagg attagatacc atactgacgc tcatgcacga aagcgtgggg agcaaacagg attagatacc acactgacgc tcatgcacga aagcgtgggg agcaaacagg attagatacc acactgacgc tcatgcacga aagcgtgggg agcaaacagg attagatacc acactgacgc tcatgcacga aagcgtgggg agcaaacagg attagatacc acactgacgc tcatgcacga aagcgtgggg agcaaacagg attagatacc atactgacgc tgaggtgcga aagcgtgggg agcaaacagg attagatacc atactgacac tgaggtgcga aagcgtgggg agcaaacagg attagatacc agactgacgc tcaggtgcga aagcgtgggg agcaaacagg attagatacc

760 770 780 790 800 ctggtagtcc acgccctaaa cgatgtcaa- ctagttgttg gg-gattcat ctggtagtcc acgccctaaa cgatgtcaa- ctagttgtcg gg-tcttcat ctggtagtcc acgccctaaa cgatgtcaa- ctagttgtcg gg-tcttcat ctggtagtcc acgccctaaa cgatgtcaa- ctagttgttg gg-gattcat ctggtagtcc acgccctaaa cgatgtcaa- ctagttgttg gg-gattcat ctggtagtcc acgccctaaa cgatgtcaa- ctagttgttg gg-gattcat 
B. cocovenenas (LMG11626) T

$B$. fungorum (LMG16225)

B. gladioli (ATCC10248) T

B. glathei (LMG14190)T

B. glathei (ATCC29195) T

B. glathei (ATCC29195)T

B. glumae (LMG2196) T

B. graminis (C4D1M) T

B. hospita (LMG20598) T

B. mallei (ATCC23344)T

B. multivorans (LMG13010) T

B. norimbergensis (Y09879) T

B. phenazinium (LMG2247) T

B. phenoliruptrix (AC1100) T

B. phymatum (STM815) T

B. phytofirmans (PsJN) T

B. pickettii (ATCC27511)T

B. pseudomallei (ATCC23343) T

B. pyrrocinia (LMG14191) T

B. pyrrocinia ATCC15958)T

B. sacchari (AF263278) T

B. silvatlantica (SRMrh-20) T

B. solanacearum (ATCC11696) T

B. sordicola (S5-B) T

B. stabilis (LMG14294) T

B. terrae (KMY02) T

B. terriccola (LMG20594) T

B. thailandensis (E264) T

B. tropicalis (Ppe8) T

B. tuberum (STM678) T

B. unamae (MT1-641) T

B. vandii (LMG16020) T

B. vietnamiensis (AMMD) $T$

B. vietnamiensis (TVV75) T

B. vietnamiensis (TVV70) T

B. plantarii (LMG9035) T

B. xenovorans (LB400) T

B. cenocepacia (KN9) T

B. ferrariae (FeG101) T

B. ambifaria (LMG19182) T

B. oklahomensis (C6786) T

B. ubonensis (GTC-P3-415) T

WC5.4C $L$. ambigua SA

WC5.4d $L$. ambigua SA

WC5.4e L. ambigua SA

WC21.1i $L$. pauciflora SA

WC21.1b L. pauciflora SA

WC21.1j L. pauciflora SA

WC26.1d L. meyerianna SA

WC26.1e L. meyerianna SA

WC26.1f L. meyerianna SA

P. lemoignei (LMG2207)

$R$. detusculanense (APF11)

$R$. solanacearum (R221)

R. pickettii (ATCC27512)

Az. Caulinodans (LMG6465)

$P$. aeruginosa (ATCC10145)

$K$. pneumoniae (AU45)

B. andropogonis (ATCC23061) T

$B$. fungorum (LMG19076) T

B. caribiensi (MWAP64) T

B. caryophylii (ATCC25418) T

B. cepacia (ATCC25416) T

B. cocovenenas (ATCC33664) T

B. cocovenenas (LMG11626) T

$B$. fungorum (LMG16225)

B. gladioli (ATCC10248)T

B. glathei (LMG14190)T

B. glathei (ATCC29195) T

B. glathei (ATCC29195)T

B. glumae (LMG2196) T

B. graminis (C4D1M) T

B. hospita (LMG20598) T

B. mallei (ATCC23344)T

B. multivorans (LMG13010) T ctggtagtcc acgccctaaa cgatgtcaa- ctagttgttg gg-gattcat ctggtagtcc acgccctaaa cgatgtcaa- ctagttgtcg gg-tcttcat ctggtagtcc acgccctaaa cgatgtcaa- ctagttgttg gg-gattcat ctggtagtcc acgccctaaa cgatgtcaa- ctagttgttg gg-gattcat ctggtagtcc acgccctaaa cgatgtcaa- ctagttgttg gg-gattcat ctggtagtcc acgccctaaa cgatgtcaa- ctagttgttg gg-gattcat ctggtagtcc acgccctaaa cgatgtcaa- ctagttgttg gg-gattcat ctggtagtcc acgccctaaa cgatgtcaa- ctggttgtcg gg-ccttcat ctggtagtcc acgccctaaa cgatgtcaa- ctagttgtco gg-tcttcat ctggtagtcc acgccccaaa cgatgtcaa- ctagttgttg gg-gattcat ctggtagtcc acgccctaaa cgatgtcaa- ctagttgttg gg-gattcat ctggtagtcc acgccctaaa cgatgtcaa- ctagttgttg gg-gattcat ctggtagtcc acgccctaaa cgatgtcaa- ctagttgtcg gg-tcttcat ctggtagtcc acgccctaaa cgatgtcaa- ctggttgtcg gg-ccttcat ctggtagtcc acgccctaaa cgatgtcaa- ctagttgtcg gg-tcttcat ctggtagtcc acgccctaaa cgatgtcaa- ctagttgtcg gg-tcttcat ctggtagtcc acgccctaaa cgatgtcaa- ctagttgttg gg-gattcat ctggtagtcc acgccctaaa cgatgtcaa- ctagttgttg gg-gattcat ctggtagtcc acgccctaaa cgatgtcaa- ctagttgttg gg-gattcat ctggtagtcc acgccctaaa cgatgtcaa- ctagttgttg gg-gattcat ctggtagtcc acgccctaaa cgatgtcaa- ctggttgtcg gg-ccttcat ctggtagtcc acgccctaaa cgatgtcaa- ctggttgtcg gg-ccttcac ctggtagtcc acgccctaaa cgatgtcaa- ctagttgttg gg-gattcat ctggtagtcc acgccctaaa cgatgtcaa- ctagttgttg gg-gattcat ctggtagtcc acgccctaaa cgatgtcaa- ctagttgttg gg-gattcat ctggtagtcc acgccctaaa cgatgtcaa- ctagttgtcg gg-tcttcat ctggtagtcc acgccctaaa cgatgtcaa- ctagttgtcg gg-ccttcat ctggtagtcc acgccctaaa cgatgtcaa- ctagttgttg gg-gattcat ctggtagtcc acgccctaaa cgatgtcaa- ctggttgtcg gg-tcttcat ctggtagtcc acgccctaaa cgatgtcaa- ctggttgtcg gg-ccttcat ctggtagtcc acgccctaaa cgatgtcaa- ctggttgtcg gg-tcttcat ctggtagtcc acgccctaaa cgatgtcaa- ctagttgttg gg-gattcat ctggtagtcc acgccctaaa cgatgtcaa- ctagttgttg gg-gattcat ctggtagtcc acgccctaaa cgatgtcaa- ctagttgttg gg-gattcat ctggtagtcc acgccctaaa cgatgtcaa- ctagttgttg gg-gattcat ctggtagtcc acgccctaaa cgatgtcaa- ctagttgttg gg-gattcat ctggtagtcc acgccctaaa cgatgtcaa- ctagttgtco gg-tcttcat ctggtagtcc acgccctaaa cgatgtcaa- ctagttgttg gg-gattcat ctggtagtcc acgccctaaa cgatgtcaa- ctggttgtcg gg-ccttcat ctggtagtcc acgccctaaa cgatgtcaa- ctagttgttg gg-gattcat ctggtagtcc acgccctaaa cgatgtcaa- ctagttgttg gg-gattcat ctggtagtcc acgccctaaa cgatgtcaa- ctagttgttg gg-gattcat ctggtagtcc acgccctaaa cgatgtcaa- ctggttgtcg gg-ccttcat ctggtagtcc acgccctaaa cgatgtcaa- ctagttgtcg gg-ccttcat ctggtagtcc acgccctaaa cgatgtcaa- ctagttgtcg gg-ccttcat ctggtagtcc acgccctaaa cgatgtcaa- ctggttgtcg gg-ccttcat ctggtagtcc acgccctaaa cgatgtcaa- ctggttgtcg gg-ccttcat ctggtagtcc acgccctaaa cgatgtcaa- ctggttgtco gg-ccttcat ctggtagtcc acgccctaaa cgatgtcaa- ctggttgtcg gg-ccttcat ctggtagtcc acgccctaaa cgatgtcaa- ctggttgtco gg-ccttcat ctggtagtcc acgccctaaa cgatgtcaa- ctggttgtcg gg-ccttcat ctggtagtcc acgccctaaa cgatgtcaa- ctagttgtcg gg-tcttaat ctggtagtcc acgccctaaa cgatgtcaa- ctagttgttg gg-gattcat ctggtagtcc acgccctaaa cgatgtcaa- ctagttgttg gg-gattcat ctggtagtcc acgccctaaa cgatgtcaa- ctagttgttg gg-gattcat ctggtagtcc acgccgtaaa cgatggatg- ctagccgttg gg-gagcttg ctggtagtcc acgccgtaaa cgatgtcga- ctagccgttg ggatccttga ctggtagtcc acgccgtaaa cgatgtcgat ttggaggttg tg-cccttga

$$
810
$$

820

830

840

850

ttccttagta acgtagctaa cgcgtgaagt tgaccgcct- ggggagtac tgacttggta acgtagctaa cgcgtgaagt tgaccgcct- ggggagtacg tgacttggta acgaagctaa cgcgtgaagt tgaccgcct- ggggagtacg ttccttagta acgaagctaa cgcgtgaagt tgaccgcct- ggggagtacg ttccttagta acgtagctaa cgcgtgaagt tgaccgcct- ggggagtacg ttccttagta acgta--taa cgcgtgaagt tgaccgcct- ggggagtacg ttccttagta acgtagctaa cgcgtgaagt tgaccgcct- ggggagtacg tgacttggta acgtagctaa cgcgtgaagt tgaccgcct- ggggagtacg ttccttagta acgtagctaa cgcgtgaagt tgaccgcct- ggggagtacg ttccttagta acgtagctaa cgcgtgaagt tgaccgcct- ggggagtacg ttccttagta acgtacgtaa cgcgtgaagt tgaccgcct- ggggagtacg ttccttagta acgta--gaa cgcgtgaagt tgaccgcct- ggggagtacg ttccttagta acgtagctaa cgcgtgaagt tgaccgcct- ggggagtacg tggcttggta acgtagctaa cgcgtgaagt tgaccgcct- ggggagtacg tgacttggta acgaagctaa cgcgtgaagt tgaccgcct- ggggagtacg ttccttagta acgtagctaa cgcgtgaagt tgaccgcct- ggggagtacg ttccttagta acgtagctaa cgcgtgaagt tgaccgcct- ggggagtacg 
B. norimbergensis (Y09879) T

B. phenazinium (LMG2247) T

B. phenoliruptrix (AC1100) T

B. phymatum (STM815) T

B. phytofirmans (PsJN) T

B. pickettii (ATCC27511)T

B. pseudomallei (ATCC23343) T

B. pyrrocinia (LMG14191) T

B. pyrrocinia ATCC15958) T

B. sacchari (AF263278)T

B. silvatlantica (SRMrh-20) T

B. solanacearum (ATCC11696) T

B. sordicola (S5-B) T

B. stabilis (LMG14294) T

B. terrae (KMY02) T

B. terriccola (LMG20594) T

B. thailandensis (E264)T

B. tropicalis (Ppe8) T

B. tuberum (STM678) T

B. unamae (MT1-641) T

B. vandi $i$ (LMG16020)T

B. vietnamiensis (AMMD) $T$

B. vietnamiensis (TVV75) T

B. vietnamiensis (TVV70)T

B. plantarii (LMG9035) T

B. xenovorans (LB400) T

B. cenocepacia (KN9) T

B. ferrariae (FeG101) T

B. ambifaria (LMG19182) T

B. oklahomensis (C6786) T

$B$. ubonensis (GTC-P3-415) T

WC5.4C L. ambigua SA

WC5.4d $L$. ambigua SA

WC5.4e $L$. ambigua SA

WC21.1i $L$. pauciflora SA

WC21.1b L. pauciflora SA

WC21.1j $L$. pauciflora SA

WC26.1d L. meyerianna SA

WC26.1e L. meyerianna SA

WC26.1f L. meyerianna SA

$P$. lemoignei (LMG2207)

$R$. detusculanense (APF11)

$R$. solanacearum (R221)

R. pickettii (ATCC27512)

Az. caulinodans (LMG6465)

$P$. aeruginosa (ATCC10145)

$K$. pneumoniae (AU45)

B. andropogonis (ATCC23061) T

$B$. fungorum (LMG19076) T

B. caribiensi (MWAP64) T

B. caryophylii (ATCC25418) T

B. cepacia (ATCC25416) T

B. cocovenenas (ATCC33664) T

B. cocovenenas (LMG11626) T

$B$. fungorum (LMG16225)

B. gladioli (ATCC10248) T

B. glathei (LMG14190) T

B. glathei (ATCC29195) T

B. glathei (ATCC29195)T

B. glumae (LMG2196) T

B. graminis (C4D1M) T

B. hospita (LMG20598) T

B. mallei (ATCC23344)T

B. multivorans (LMG13010) T

$B$. norimbergensis (Y09879) T

B. phenazinium (LMG2247) T

B. phenoliruptrix (AC1100) T

B. phymatum (STM815) T

B. phytofirmans (PsJN) T

B. pickettii (ATCC27511)T

B. pseudomallei (ATCC23343) T

B. pyrrocinia (LMG14191) T

B. pyrrocinia ATCC15958) T

B. sacchari (AF263278) T

B. silvatlantica (SRMrh-20) T ttccttagta acgaagctaa cgcgtgaagt tgaccgcct- ggggagtacg tgacttggta acgtagctaa cgcgtgaagt tgaccgcct- ggggagtacg tggcttggta acgtagctaa cgcgtgaagt tgaccgcct- ggggagtacg tgacttggta acgtagctaa cgcgtgaagt tgaccgcct- ggggagtacg tgacttggta acgtagctaa cgcgtgaagt tgaccgcct- ggggagtacg ttccttagta acgtagctaa cgcgtgaagt tgaccgcct- ggggagtacg ttccttagta acgtagctaa cgcgtgaagt tgaccgcct- ggggagtacg ttccttagta acgtagctaa cgcgtgaagt tgaccgcct- ggggagtacg ttccttagta acgtagctaa cgcgtgaagt tgaccgcct- ggggagtacg tggcttggta acgtagctaa cgcgtgaagt tgaccgcct- ggggagtacg tggcttggta acgtagctaa cgcgtgaagt tgaccgcct- ggggagtacg ttccttagta acgtagctaa cgcgtgaagt tgaccgcct- ggggagtacg ttccttagta acgtagctaa cgcgtgaagt tgaccgcct- ggggagtacg ttccttagta acgtagctaa cgcgtgaagt tgaccgcct- ggggagtacg tgacttggta acgaagctaa cgcgtgaagt tgaccgcct- ggggagtacg tggcttggta acgtagctaa cgcgtgaagt tgaccgcct- ggggagtacg ttccttagta acgtagctaa cgcgtgaagt tgaccgcct- ggggagtacg tgacttggta acgtagctaa cgcgtgaagt tgaccgcct- ggggagtacg tggcttggta acgtagctaa cgcgtgaagt tgaccgcct- ggggagtacg tgacttggta acgtagctaa cgcgtgaagt tgaccgcct- ggggagtaco ttccttagta acgtagctga cgcgtgaagt tgaccgcct- ggggagtacg ttccttagta acgtagctaa cgcgtgaagt tgaccgcct- ggggagtacg ttcctagta acgtagctaa cgcgtgaagt tgaccgcct- ggggagtacg ttccttagta acgtagctaa cgcgtgaagt tgaccgcct- ggggagtacg ttccttagta acgtagctaa cgcgtgaagt tgaccgcct- ggggagtacg tgacttggta acgtagctaa cgcgtgaagt tgaccgcct- ggggagtacg ttccttagta acgtagctaa cgcgtgaagt tgaccgcct- ggggagtacg tggcttggta acgtagctaa cgcgtgaagt tgaccgcct- ggggagtacg ttccttagta acgtagctaa cgcgtgaagt tgaccgcct- ggggagtacg ttccttagta acgtagctaa cgcgtgaagt tgaccgcct- ggggagtacg ttccttagta acgtagctaa cgcgtgaagt tgaccgcct- ggggagtacg tggcttggta acgtagctaa cgcgtgaagt tgaccgcct- ggggagtacg tggcttggta acgtagctaa cgcgtgaagt tgaccgcct- ggggagtacg tggcttggta acgtagctaa cgcgtgaagt tgaccgcct- ggggagtaco tggcttggta acgaagctaa cgcgtgaagt tgaccgcct- ggggagtacg tggcttggta acgaagctaa cgcgtgaagt tgaccgcctg ggggagtacg tggcttggta acgaagctaa cgcgtgaagt tgaccgcct- ggggagtaco tggctggtta acgaagctaa cgcgtgaagt tgaccgcct- tgggagtacg tggcttggta acgaagctaa cgcgtgaagt tgaccgcct- ggggagtacg tggcttggta acgaagctaa cgcgtgaagt tgaccgcct- ggggagtacg tgacttggta acgcagctaa cgcgtgaagt tgaccgcct- ggggagtacg ttccttagta acgtagctaa cgcgtgaagt tgaccgcct- ggggagtacg ttccttagta acgtagctaa cgcgtgaagt tgaccgcct- ggggagtacg ttccttagta acgtagctaa cgcgtgaagt tgaccgcct- ggggagtacg ctcttcagtg gcgcagctaa cgccttaagc atcccgcct- ggggagtacg gatcttagtg gcgcagctaa cgcgataagt cgaccgcct- ggggagtacg ggcgtggctt ccggagctaa cgcgttaaat cgaccgcct- ggggagtacg

860 870

880

890

900

gtcgcaagat taaaactcaa aggaattgac ggggacccgc acaagcggtg gtcgcaagat taaaactcaa aggaattgac ggggacccgc acaagcggtg gtcgcaagat taaaactcaa aggaattgac ggggacccgc acaagcggtg gtcgcaagat taaaactcaa aggaattgac ggggacccgc acaagcggtg gtcgcaagat taaaactcaa aggaattgac ggga--ccgc acaagcggtg gtcgcaagat taaaactcaa aggaattgac ggggacccgc acaagcggtg gtcgcaagat taaaactcaa aggaattgac ggggacccgc acaagcggtg gtcgcaagat taaaactcaa aggaattgac ggggacccgc acaagcggtg gtcgcaagat taaaactcaa aggaattgac ggggacccgc acaagcggtg gtcgcaagat taaaactcaa aggaattgac ggggacccgc acaagcggtg gtcgcaagat taaaactcaa aggaattgac ggggacccgc acaagcggtg gtcgcaagat taaaactcaa aggaattgac ggggacccgc acaagcggtg gtcgcaagat taaaactcaa aggaattgac ggggacccgc acaagcggtg gtcgcaagat taaaactcaa aggaattgac ggggacccgc acaagcggtg gtcgcaagat taaaactcaa aggaattgac ggggacccgc acaagcggtg gtcgcaagat taaaactcaa aggaattgac ggggacccgc acaagcggtg gtcgcaagat taaaactcaa aggaattgac ggggacccgc acaagcggtg gtcgcaagat taaaactcaa aggaattgac ggggacccgc acaagcggtg gtcgcaagat taaaactcaa aggaattgac ggggacccgc acaagcggtg gtcgcaagat taaaactcaa aggaattgac ggggacccgc acaagcggtg gtcgcaagat taaaactcaa aggaattgac ggggacccgc acaagcggtg gtcgcaagat taaaactcaa aggaattgac ggggacccgc acaagcggtg gtcgcaagat taaaactcaa aggaattgac ggggacccgc acaagcggtg gtcgcaagat taaaactcaa aggaattgac ggggacccgc acaagcggtg gtcgcaagat taaaactcaa agg-attgac gggga-ccgc acaagcggtg gtcgcaagat taaaactcaa aggaattgac ggggacccgc acaagcggtg gtcgcaagat taaaactcaa aggaattgac ggggacccgc acaagcggtg gtcgcaagat taaaactcaa aggaattgac ggggacccgc acaagcggtg 
B. solanacearum (ATCC11696) T

B. sordicola (S5-B) T

B. stabilis (LMG14294)T

$B$. terrae (KMY02) T

B. terriccola (LMG20594) T

$B$. thailandensis (E264) T

B. tropicalis (Ppe8) T

B. tuberum (STM678) T

B. unamae (MT1-641) T

B. vandii (LMG16020) T

$B$. vietnamiensis (AMMD) T

B. vietnamiensis (TVV75) T

B. vietnamiensis (TVV70)T

B. plantarii (LMG9035) T

B. xenovorans (LB400) T

B. cenocepacia (KN9) T

$B$. ferrariae (FeG101) T

B. ambifaria (LMG19182) T

B. oklahomensis (C6786) T

B. ubonensis (GTC-P3-415) T

WC5.4C $L$. ambigua SA

WC5.4d $L$. ambigua SA

WC5.4e $L$. ambigua SA

WC21.1i L. pauciflora SA

WC21.1b $L$. pauciflora SA

WC21.1j $L$. pauciflora SA

WC26.1d $L$. meyerianna SA

WC26.1e $L$. meyerianna SA

WC26.1f $L$. meyerianna SA

$P$. lemoignei (LMG2207)

$R$. detusculanense (APF11)

$R$. solanacearum (R221)

R. pickettii (ATCC27512)

Az. caulinodans (LMG6465)

$P$. aeruginosa (ATCC10145)

$K$. pneumoniae (AU45)

B. andropogonis (ATCC23061) T

$B$. fungorum (LMG19076) T

B. caribiensi (MWAP64) T

B. caryophylii (ATCC25418)T

B. cepacia (ATCC25416) T

B. cocovenenas (ATCC33664)T

B. cocovenenas (LMG11626) T

$B$. fungorum (LMG16225)

B. gladioli (ATCC10248) T

B. glathei (LMG14190) T

B. glathei (ATCC29195)T

B. glathei (ATCC29195) T

B. glumae (LMG2196) T

$B$. graminis (C4D1M) T

B. hospita (LMG20598) T

B. mallei (ATCC23344) T

B. multivorans (LMG13010) T

$B$. norimbergensis (Y09879) T

B. phenazinium (LMG2247) T

B. phenoliruptrix (AC1100) T

B. phymatum (STM815) T

B. phytofirmans (PsJN) T

B. pickettii (ATCC27511)T

B. pseudomallei (ATCC23343) T

B. pyrrocinia (LMG14191) T

B. pyrrocinia ATCC15958) T

B. sacchari (AF263278) T

$B$. silvatlantica (SRMrh-20)T

B. solanacearum (ATCC11696) T

B. sordicola (S5-B) T

B. stabilis (LMG14294) T

B. terrae (KMY02) T

B. terriccola (LMG20594) T

B. thailandensis (E264) T

B. tropicalis (Ppe8) T

B. tuberum (STM678) T

B. unamae (MT1-641) T

B. vandii (LMG16020) T

$B$. vietnamiensis (AMMD) T gtcgcaagat taaaactcaa aggaattgac ggggacccgc acaagcggtg gtcgcaagat taaactcaa aggaattgac ggggacccgc acaagcggtg gtcgcaagat taaaactcaa aggaattgac ggggacccgc acaagcggtg gtcgcaagat taaaactcaa aggaattgac ggggacccgc acaagcggtg gtcgcaagat taaactcaa aggaattgac ggggacccgc acaagcggtg gtcgcaagat taaaactcaa aggaattgac ggggacccgc acaagcggtg gtcgcaagat taaaactcaa aggaattgac ggggacccgc acaagcggtg gtcgcaagat taaactcaa aggaattgac ggggacccgc acaagcggtg gtcgcaagat taaaactcaa aggaattgac ggggacccgc acaagcggtg gtcgcaagat taaactcaa aggaattgac ggggacccgc acaagcggtg gtcgcaagat taaaactcaa aggaattgac ggggacccgc acaagcggtg gtcgcaagat taaaactcaa aggaattgac ggggacccgc acaagcggtg gtcgcaagat taaactcaa aggaattgac ggggacccgc acaagcggtg gtcgcaagat taaaactcaa aggaattgac ggggacccgc acaagcggtg gtcgcaagat taaaactcaa aggaattgac ggggacccgc acaagcggtg gtcgcaagat taaaactcaa aggaattgac ggggacccgc acaagcggtg gtcgcaagat taaaactcaa aggaattgac ggggacccgc acaagcggtg gtcgcaagat taaaactcaa aggaattgac ggggacccgc acaagcggtg gtcgcaagat taaactcaa aggaattgac ggggacccgc acaagcggtg gtcgcaagat taaaactcaa aggaattgac ggggacccgc acaagcggto gtcgcaagat taaactcaa aggaattgac ggggacccgc acaagcggtg gtcgcaagat taaaactcaa aggaattgac ggggacccgc acaagcggtg gtcgcaagat taaaactcaa aggaattgac ggggacccgc acaagcggtg gtcgcaagat taaactcaa aggaattgac ggggacccgc acaagcggtg gtcgcaagat taaaactcaa aggaattgac ggggacccgc acaagcggtg gtcgcaagat taaaactcaa aggaattgac ggggacccgc acaagcggto gtcgcaagat twaaactcaa aggaattgac ggggacccgc acaagcggtg gtcgcaagat taaaactcaa aggaattgac ggggacccgc acaagcggtg gtcgcaagat taaaactcaa aggaattgac ggggacccgc acaagcggtg gtcgcaagat taaactcaa aggaattgac ggggacccgc acaagcggtg gtcgcaagat taaaactcaa aggaattgac ggggacccgc acaagcggto gtcgcaagat taaaactcaa aggaattgac ggggacccgc acaagcggtg gtcgcaagat taaactcaa aggaattgac ggggacccgc acaagc-gtg gtcgcaagat taaaactcaa aggaattgac ggggg-ccgc acaagcggtg gccgcaaggt taaaactcaa atgaattgac gggggcccgc acaagcggtg gccgcaaggt taaaactcaa atgaattgac gggggcccgc acaagcggtg

910 920 930 940

950

gatgatgtgg attaattcga tgcaacgcga aaacc-tta ccta-ccct gatgatgtgg attaattcga tgcaacgcga aaacc-tta ccta-ccctt gatgatgtgg attaattcga tgcaacgcga aaacc-tta ccta-ccct gatgatgtgg attaattcga tgcaacgcga aaacc-tta ccta-ccctt gatgatgtgg attaattcga tgcaacgcga aaacc-tta ccta-ccctt gatgatgtgg attaattcga tgcaacgcga aaacc-tta ccta-ccct gatgatgtgg attaattcga tgcaacgcga aaacc-tta ccta-ccctt gatgatgtgg attaattcga tgcaacgcga aaacc-tta ccta-ccctt gatgatgtgg attaattcga tgcaacgcga aaacc-tta ccta-ccctt gatgatgtgg attaattcga tgcaacgcga aaacc-tta ccta-ccctt gatgatgtgg attaattcga tgcaacgcga aaacc-tta ccta-ccct gatgatgtgg attaattcga tgcaacgcga aaacc-tta ccta-ccctt gatgatgtgg attaattcga tgcaacgcga aaacc-tta ccta-ccctt gatgatgtgg attaattcga tgcaacgcga aaacc-tta ccta-ccct gatgatgtgg attaattcga tgcaacgcga aaacc-tta ccta-ccctt gatgatgtgg attaattcga tgcaacgcga aaacc-tta ccta-ccct gatgatgtgg attaattcga tgcaacgcga aaacc-tta ccta-ccctt gatgatgtgg attaattcga tgcaacgcga aaacc-tta ccta-ccctt gatgatgtgg attaattcga tgcaacgcga aaacc-tta ccta-ccctt gatgatgtgg attaattcga tgcaacgcga aaacc-tta ccta-ccctt gatgatgtgg attaattcga tgcaacgcga aaacc-tta ccta-ccctt gatgatgtgg attaattcga tgcaacgcga aaacc-tta ccta-ccctt gatgatgtgg attaattcga tgcaacgcga aaacc-tta ccta-ccctt gatgatgtgg attaattcga tgcaacgcga aaaacc-tta ccta-ccctt gatgatgtgg attaattcga tgcaacgcga aaacc-tta ccta-ccctt gatgatgtgg attaattcga tgcaacgcga aaacc-tta ccta-ccctt gatgatgtgg attaattcga tgcaacgcga aaacc-tta ccta-ccctt gatgatgtgg attaattcga tgcaacgcga aaacc-tta ccta-ccctt gatgatgtgg attaattcga tgcaacgcga aaacc-tta ccta-ccctt gatgatgtgg attaattcga tgcaacgcga aaacc-tta ccta-ccct gatgatgtgg attaattcga tgcaacgcga aaacc-tta ccta-ccctt gatgatgtgg attaattcga tgcaacgcga aaacc-tta ccta-ccctt gatgatgtgg attaattcga tgcaacgcga aaacc-tta ccta-ccctt gatgatgtgg attaattcga tgcaacgcga aaacc-tta ccta-ccctt gatgatgtgg attaattcga tgcaacgcga aaacc-tta ccta-ccctt gatgatgtgg attaattcga tgcaacgcga aaacc-tta ccta-ccct gatgatgtgg attaattcga tgcaacgcga aaacc-tta ccta-ccctt gatgatgtgg attaattcga tgcaacgcga aaacc-tta ccta-ccct gatgatgtgg attaattcga tgcaacgcga aaacc-tta ccta-ccct 
B. vietnamiensis (TVV75)T

B. vietnamiensis (TVV70) T

B. plantarii (LMG9035) T

B. xenovorans (LB400) T

$B$. cenocepacia (KN9) T

B. ferrariae (FeG101) T

B. ambifaria (LMG19182) T

$B$. oklahomensis (C6786) T

B. ubonensis (GTC-P3-415) T

WC5.4C $L$. ambigua SA

WC5.4d $L$. ambigua SA

WC5.4e $L$. ambigua SA

WC21.1i $L$. pauciflora SA

WC21.1b $L$. pauciflora SA

WC21.1j $L$. pauciflora SA

WC26.1d $L$. meyerianna SA

WC26.1e $L$. meyerianna SA

WC26.1f $L$. meyerianna SA

$P$. lemoignei (LMG2207)

$R$. detusculanense (APF11)

R. solanacearum (R221)

R. pickettii (ATCC27512)

Az. caulinodans (LMG6465)

$P$. aeruginosa (ATCC10145)

$K$. pneumoniae (AU45)

B. andropogonis (ATCC23061) T

$B$. fungorum (LMG19076) $T$

B. caribiensi (MWAP64) T

B. caryophylii (ATCC25418)T

B. cepacia (ATCC25416) T

B. cocovenenas (ATCC33664) T

B. cocovenenas (LMG11626) T

B. fungorum (LMG16225)

B. gladioli (ATCC10248) T

B. glathei (LMG14190) T

B. glathei (ATCC29195) T

B. glathei (ATCC29195) T

B. glumae (LMG2196) T

$B$. graminis (C4D $1 \mathrm{M}) \mathrm{T}$

B. hospita (LMG20598) T

B. mallei (ATCC23344) T

B. multivorans (LMG13010) T

B. norimbergensis (Y09879) T

B. phenazinium (LMG2247)T

B. phenoliruptrix (AC1100) T

B. phymatum (STM815) T

B. phytofirmans (PsJN) T

B. pickettii (ATCC27511) T

B. pseudomallei (ATCC23343) T

B. pyrrocinia (LMG14191) T

B. pyrrocinia ATCC15958) T

B. sacchari (AF263278) T

B. silvatlantica (SRMrh-20)T

B. solanacearum (ATCC11696) T

$B$. sordicola (S5-B) $T$

B. stabilis (IMG14294) T

B. terrae (KMY02) T

B. terriccola (LMG20594) T

B. thailandensis (E264) T

$B$. tropicalis (Ppe8) T

B. tuberum (STM678) T

B. unamae (MT1-641) T

$B$. vandii (LMG16020) T

$B$. vietnamiensis (AMMD) T

B. vietnamiensis (TVV75) T

B. vietnamiensis (TVV70)T

B. plantarii (LMG9035) T

B. xenovorans (LB400) T

$B$. cenocepacia (KN9) T

B. ferrariae (FeG101) T

B. ambifaria (LMG19182) T

B. oklahomensis (C6786) T

B. ubonensis (GTC-P3-415) T

WC5.4C $L$. ambigua SA

WC5.4d $L$. ambigua SA gatgatgtgg attaattcga tgcaacgcga aaacc-tta ccta-ccctt gatgatgtgg attaattcga tgcaacgcga aaacc-tta ccta-ccctt gatgatgtgg attaattcga tgcaacgcga aaaacc-tta ccta-ccctt gatgatgtgg attaattcga tgcaacgcga aaaacc-tta ccta-ccctt gatgatgtgg attaattcga tgcaacgcga aaacc-tta ccta-ccctt gatgatgtgg attaattcga tgcaacgcga aaacc-tta ccta-ccctt gatgatgtgg attaattcga tgcaacgcga aaacc-tta ccta-ccctt gatgatgtgg attaattcga tgcaacgcga aaacc-tta ccta-ccct gatgatgtgg attaattcga tgcaacgcga aaacc-tta ccta-ccctt gatgatgtgg attaattcga tgcaacgcga aaacc-tta ccta-ccctt gatgatgtgg attaattcga tgcaacgcga aaacc-tta ccta-ccctt gatgatgtgg attaattcga tgcaacgcga aaacc-tta ccta-ccctt gatgatgtgg attaattcga tgcaacgcga aaacc-tta ccta-ccctt gatgatgtgg attaattcga tgcaacgcga aaacc-tta ccta-ccctt gatgatgtgg attaattcga tgcaacgcga aaacc-tta cctacccctt gatgatgtgg attaattcga tgcaacgcga aaaccttta ccta-ccctt gatgatgtgg attaattcga tgcaacgcga aaacc-tta ccta-ccctt gatgatgtgg attaattcga tgcaacgcga aaacc-tta ccta-ccct gatgatgtgg attaattcga tgcaacgcga aaacc-tta ccta-ccct gatgatgtgg attaattcga tgcaacgcga aaacc-tta ccta-ccctt gatgatgtgg attaattcga tgcaacgcga aaacc-tta ccta-ccctt gatgatgtgg attaattcga tgcaacgcga aaacc-tta ccta-ccctt gagcatgtgg tttaattcga agcaacgcgc agaacc-tta ccag-cctt gagcatgtgg tttaattcga agcaacgcga agaacc-tta cctg-gcctt gagcatgtgg tttaattcga tgcaacgcga agaacc-tta cctg-gtctt

960 970

980

990

1000

gacatggtcg gaacccttga gagatcag-g gggtgctcga aagagaaccg gacatgtatg gaaccctgct gagaggtg-g gggtgcccga aagggagcca gacatgtacg gaaccttgct gagaggtg-a gggtgcccga aagggagccg gacatggacg gaatcccgct gagaggtg-g gagtgctcga aagagaaccg gacatggtcg gaatcctgct gagaggcg-g gagtgctcga aagagaaccg gacatggtcg gaatcctgga gagatctg-g gagtgctcga aagagaaccg gacatggtcg gaatcctgga gagatctg-g gagtgctcga aagagaaccg gacatgtatg gaatcctgct gagaggtg-g gagtgcccga aagggagcca gacatggtcg gaatcctaga gagatctg-g gagtgctcga aagagaaccg gacatggtcg gaaccctggt gagagctg-g gggtgctcga aagagaaccg gacatggtcg gaaccctggt gagagctg-g gggtgctcga aagagaaccg gacatggtcg gaaccctggt gagagctg-g gggtgctcga aagagaaccg gacatggtcg gaatcctgag ga-actcg-g gagtgctcga aagagaaccg gacatgtacg gaagtccgct gagaggtg-g atgtgcccga aagggagccg gacatgtacg gaaccttgcc gagaggtg-a gggtgcccga aagggagccg gacatggtcg gaagcccgat gagagttg-g gcgtgctcga aagagaaccg gacatggtcg gaatcctgaa gagattcg-g gagtgctcga aagagaaccg gacatgtacg gaatcttgct gaaaggtg-a gagtgctcga aagagagccg gacatggtcg gaaccctgct gaaaggtg-g gggtgctcga aagagaaccg gacatggatg gaatcccgct gagaggtg-g gagtgcccga aagggagcca gacatgtacg gaaccttgcc gagaggtg-a gggtgcccga aagggagccg gacatgtatg gaatcctgct gagaggtg-g gagtgcccga aagggagcca gacatgccac taacgaagca gagatgcatt aggtgctcga aagagaaagt gacatggtcg gaagcccgat gagagttg-g gcgtgctcga aagagaaccg gacatggtcg gaatcctgct gagaggtg-g gagtgctcga aagagaaccg gacatggtcg gaatcctgct gagaggtg-g gagtgctcga aagagaaccg gacatgtacg gaatcctgct gagaggcg-g gagtgcccga aagggagccg gacatggacg gaagaccggt gagagctg-g ttgtgcccga aagggagccg gacatgccac taacgaagca gagatgcatt aggtgctcga aagagaaagt gacatggtcg gaacctggct gaaaggct-g gggtgctcga aagagaaccg gacatggtcg gaatcctgct gagaggtg-g gagtgctcga aagagaaccg gacatgtacg gaaccttgct gagaggtg-a gggtgcccga aagggagccg gacatgtacg gaatcctgct gagaggcg-g gagtgcccga aagggagccg gacatggtcg gaatcctgct gagaggcg-g gagtgctcga aagagaaccg gacatgtacg gaattccgct gagaggtg-g aagtgcccga aagggagccg gacatgtatg gaatcctgcc gagaggcg-g gagtgcccga aagggagccg gacatgtatg gaatcctgct gagaggtg-g gagtgcccga aagggagcca gacatggtcg gaatcctgag gagactcg-g gagtgctcga aagagaaccg gacatggtcg gaatcctgct gagaggtg-g gagtgctcga aagagaaccg gacatggtcg gaatcctgaa gagattcg-g gagtgctcga aagagaaccg gacatggtcg gaatcctgaa gagattcg-g gagtgctcga aagagaaccg gacatggtcg gaatcccgaa gagatttg-g gagtgctcga aagagaaccg gacatgtatg gaagtctgct gagaggtg-g atgtgcccga aagggagcca gacatggtcg gaatcctgcc gagaggtg-g gagtgctcga aagagaaccg gacatgtacg gaagtctgcc gagaggtg-g atgtgcccga aagggagccg gacatggtcg gaatcctgct gagagggg-g gagtgctcga aagagaaccg gacatggtcg gaatcctgct gagaggtg-g gagtgctcga aagagaaccg gacatggtcg gaatcctgaa gagattcg-g gagtgctcga aagagaaccg gacatgtatg gaatcctgcc gagaggcg-g gagtgcccga aagggagcca gacatgtatg gaacctggct gagaggtc-a gggtgcccga aagggagcca 
WC5.4e $L$. ambigua SA WC21.1i L. pauciflora SA WC21.1b $L$. pauciflora SA WC21.1j $L$. pauciflora SA WC26.1d $L$. meyerianna SA WC26.1e $L$. meyerianna SA WC26.1f $L$. meyerianna SA $P$. lemoignei (LMG2207)

$R$. detusculanense (APF11)

$R$. solanacearum (R221)

R. pickettii (ATCC27512)

Az. caulinodans (LMG6465)

$P$. aeruginosa (ATCC10145)

$K$. pneumoniae (AU45)

B. andropogonis (ATCC23061) T

$B$. fungorum (LMG19076) $T$

B. caribiensi (MWAP64) T

B. caryophylii (ATCC25418) T

B. cepacia (ATCC25416) T

B. cocovenenas (ATCC33664) T

$B$. cocovenenas (LMG11626) T

$B$. fungorum (LMG16225)

B. gladioli (ATCC10248) T

B. glathei (LMG14190) T

B. glathei (ATCC29195)T

B. glathei (ATCC29195) T

B. glumae (LMG2196) T

$B$. graminis (C4D1M) T

B. hospita (LMG20598) T

B. mallei (ATCC23344) T

B. multivorans (LMG13010) T

$B$. norimbergensis (Y09879) T

B. phenazinium (LMG2247) T

B. phenoliruptrix (AC1100) T

B. phymatum (STM815) T

B. phytofirmans (PsJN) T

B. pickettii (ATCC27511)T

B. pseudomallei (ATCC23343) T

B. pyrrocinia (LMG14191) T

B. pyrrocinia ATCC15958)T

B. sacchari (AF263278) T

B. silvatlantica (SRMrh-20) T

B. solanacearum (ATCC11696) T

$B$. sordicola (S5-B) T

B. stabilis (LMG14294) T

B. terrae (KMY02) T

B. terriccola (LMG20594) T

B. thailandensis (E264) T

B. tropicalis (Ppe8) T

B. tuberum (STM678) T

B. unamae (MT1-641) T

$B$. vandii (LMG16020) T

$B$. vietnamiensis (AMMD) T

B. vietnamiensis (TVV75) T

B. vietnamiensis (TVV70)T

B. plantarii (LMG9035) T

B. xenovorans (LB400) T

$B$. cenocepacia (KN9) T

$B$. ferrariae (FeG101) T

B. ambifaria (LMG19182) T

$B$. oklahomensis (C6786) T

B. ubonensis (GTC-P3-415) T

WC5.4C $L$. ambigua SA

WC5.4d $L$. ambigua SA

WC5.4e $L$. ambigua SA

WC21.1i L. pauciflora SA

WC21.1b $L$. pauciflora SA

WC21.1j $L$. pauciflora SA

WC26.1d $L$. meyerianna SA

WC26.1e $L$. meyerianna SA

WC26.1f $L$. meyerianna SA

$P$. lemoignei (LMG2207)

$R$. detusculanense (APF11)

R. solanacearum (R221)

R. pickettii (ATCC27512) gacatgtatg gaacctggct gagaggtc-a gggtgcccga aagggagcca gacatgtatg gaatcctggc gagagccg-g gggtgcccga aagggagcca gacatgtatg gaatcctggc gagagccg-g gagtgcccga aagggagcca gacatgtatg gaatcctggt gagagccg-g gagtgcccga aagggagcca gacatgtatg gaatcctggt gagagccg-g gagtgcccga aagggagcca gacatgtatg gaatcctggt gagagccg-g gagtgcccga aagggagcca gacatgtatg gaatcctggt gagagccg-g gagtgcccga aagggagcca gacatgtacg gaatccttca gagatgga-g gagtgctcga aagagagccg gacatgccac taacgaagca gagatgcatt aggtgctcga aagagaaagt gacatgccac taacgaagca gagatgcatt aggtg-tcga aagagaaagt gacatgccac taacgaagca gagatgcatt aggtgctcga aagagaaagt gacatggcag gacgacttcc ggagacgg-a tttcttccag caatggacct gacatgctga gaactttcca gagatgga-t tggtgcct-- tcgggaactc gacatccaca gaactttcca gagatgga-t tggtgcct-- tcgggaactg

$$
1010
$$

1020

1030

1040

1050

atacacaggt gctgcatggc tgtcgtc-ag ctcotgtcgt gagatgttgg taacacaggt gctgcatggc tgtcgtc-ag ctcgtgtcgt gagatgttgg taacacaggt gctgcatggc tgtcgtc-ag ctcgtgtcgt gagatgttgg tcgcacaggt gctgcatggc tgtcgtc-ag ctcgtgtcgt gagatgttgg gcgcacaggt gctgcatggc tgtcgtc-ag ctcgtgtcgt gagatgttgg atacacaggt gctgcatggc tgtcgtc-ag ctcgtgtcgt gagatgttgg atacacaggt gctgcatggc tgtcgtc-ag ctcgtgtcgt gagatgttgg taacacaggt gctgcatggc tgtcgtc-ag ctcgtgtcgt gagatgttgg atacacaggt gctgcatggc tgtcgtc-ag ctcgtgtcgt gagatgttgg acacacaggt gctgcatggc tgtcgtc-ag ctcgtgtcgt gagatgttgg acacacaggt gctgcatggc tgtcgtc-ag ctcgtgtcgt gagatgttgg acacacaggt gctgcatggc tgtcgtc-ag ctcgtgtcgt gagatgttgg atacacaggt gctgcatggc tgtcgtc-ag ctcgtgtcgt gagatgttgg taacacaggt gctgcatggc tgtcgtc-ag ctcgtgtcgt gagatgttgg taacacaggt gctgcatggc tgtcgtc-ag ctcgtgtcgt gagatgttgg gcgcacaggt gctgcatggc tgtcgtc-ag ctcgtgtcgt gagatgttgg gcgcacaggt gctgcatggc tgtcgtc-ag ctcgtgtcgt gagatgttgg taacacaggt gctgcatggc tgtcgtc-ag ctcgtgtcgt gagatgttgg atacacaggt gctgcatggc tgtcgtc-ag ctcgtgtcgt gagatgttgg tcacacaggt gctgcatggc tgtcgtc-ag ctcgtgtcgt gagatgttgg taacacaggt gctgcatggc tgtcgtc-ag ctcgtgtcgt gagatgttgg taacacaggt gctgcatggc tgtcgtc-ag ctcgtgtcgt gagatgttgg ggacacaggt gctgcatggc tgtcgtc-ag ctcgtgtcgt gagatgttgg gcgcacaggt gctgcatggc tgtcgtc-ag ctcgtgtcgt gagatgttgg atacacaggt gctgcatggc tgtcgtc-ag ctcgtgtcgt gagatgttgg atacacaggt gctgcatggc tgtcgtc-ag ctcgtgtcgt gagatgttgg taacacaggt gctgcatggc tgtcgtc-ag ctcgtgtcgt gagatgttgg tcacacaggt gctgcatggc tgtcgtc-ag ctcgtgtcgt gagatgttgg ggacacaggt gctgcatggc tgtcgtc-ag ctcgtgtcgt gagatgttgg atacacaggt gctgcatggc tgtcgtc-ag ctcgtgtcgt gagatgttgg atacacaggt gctgcatggc tgtcgtc-ag ctcgtgtcgt gagatgttgg taacacaggt gctgcatggc tgtcgtc-ag ctcgtgtcgt gagatgttgg taacacaggt gctgcatggc tgtcgtc-ag ctcgtgtcgt gagatgttgg gcgcacaggt actgcatggc tgtcgtc-ag ctcgtgtcgt gagatgttgg taacacaggt gctgcatggc tgtcgtc-ag ctcgtgtcgt gagatgttgg taacacaggt gctgcatggc tgtcgtc-ag ctcgtgtcgt gagatgttgg taacacaggt gctgcatggc tgtcgtc-ag ctcgtgtcgt gagatgttgg atacacaggt gctgcatggc tgtcgtc-ag ctcgtgtcgt gagatgttgg gcgcacaggt gctgcatggc tgtcgtc-ag ctcgtgtcgt gagatgttgg gcgcacaggt gctgcatggc tgtcgtc-ag ctcgtgtcgt gagatgttgg gcgcacaggt gctgcatggc tgtcgtc-ag ctcgtgtcgt gagatgttgg atacacaggt gctgcatggc tgtcgtc-ag ctcgtgtcgt gagatgttgg taacacaggt gctgcatggc tgtcgac-ag ctcgtgtcgt gagatgttgg gcgcacaggt gctgcatggc tgtcgtc-ag ctcgtgtcgt gagatgttgg taacacaggt gctgcatggc tgtcgtc-ag ctcgtgtcgt gagatgttgg gcgcacaggt gctgcatggc tgtcgtc-ag ctcgtgtcgt gagatgttgg atacacaggt gctgcatggc tgtcgtc-ag ctcgtgtcgt gagatgttgg gcgcacaggt gctgcatggc tgtcgtc-ag ctcgtgtcgt gagatgttgg taacacaggt gctgcatggc tgtcgtc-ag ctcgtgtcgt gagatgttgg taacacaggt gctgcatggc tgtcgtc-ag ctcgtgtcgt gagatgttgg taacacaggt gctgcatggc tgtcgtc-ag ctcgtgtcgt gagatgttgg taacacaggt gctgcatggc tgtcgtc-ag ctcgtgtcgt gagatgttgg taacacaggt gctgcatggc tgtcgtc-ag ctcgtgtcgt gagatgttgg taacacaggt gctgcatggc tgtcgtc-ag ctcgtgtcgt gagatgttgg taacacaggt gctgcatggc tgtcgtcaag ctcgtgtcgt gagatgttgg taacacaggt gctgcatggc tgtcgtc-ag ctcgtgtcgt gagatgttgg taacacaggt gctgcatggc tgtcgtc-ag ctcgtgtcgt gagatgttgg taacacaggt gctgcatggc tgtcgtc-ag ctcgtgtcgt gagatgttgg ggacacaggt gctgcatggc tgtcgtc-ag ctcgtgtcgt gagatgttgg ggacacaggt gctgcatggc tgtcgtc-ag ctcgtgtcgt gagatgttgg ggacacaggt gctgcatggc tgtcgtc-ag ctcgtgtcgt gagatgttgg 
Az. Caulinodans (LMG6465)

$P$. aeruginosa (ATCC10145)

$K$. pneumoniae (AU45)

B. andropogonis (ATCC23061) T

$B$. fungorum (LMG19076) T

B. caribiensi (MWAP64) T

B. caryophylii (ATCC25418) T

B. cepacia (ATCC25416) T

B. cocovenenas (ATCC33664)T

B. cocovenenas (LMG11626) T

$B$. fungorum (LMG16225)

B. gladioli (ATCC10248) T

B. glathei (LMG14190) T

B. glathei (ATCC29195)T

B. glathei (ATCC29195) T

B. glumae (LMG2196) T

$B$. graminis (C4D1M) T

B. hospita (LMG20598) T

B. mallei (ATCC23344) T

B. multivorans (LMG13010) T

$B$. norimbergensis (Y09879) T

B. phenazinium (LMG2247) T

B. phenoliruptrix (AC1100) T

B. phymatum (STM815) T

B. phytofirmans (PsJN) T

B. pickettii (ATCC27511) T

B. pseudomallei (ATCC23343) T

B. pyrrocinia (LMG14191)T

B. pyrrocinia ATCC15958) T

B. sacchari (AF263278) T

B. silvatlantica (SRMrh-20)T

B. solanacearum (ATCC11696) T

$B$. sordicola (S5-B) $\mathrm{T}$

B. stabilis (LMG14294) T

B. terrae (KMY02) T

B. terriccola (LMG20594) T

$B$. thailandensis (E264)T

B. tropicalis (Ppe8) T

B. tuberum (STM678) T

B. unamae (MT1-641) T

B. vandii (LMG16020) T

$B$. vietnamiensis (AMMD) T

B. vietnamiensis (TVV75) T

B. vietnamiensis (TVV70)T

B. plantarii (LMG9035) T

$B$. xenovorans (LB400) T

$B$. cenocepacia (KN9) T

$B$. ferrariae (FeG101) T

B. ambifaria (LMG19182) T

B. oklahomensis (C6786) T

B. ubonensis (GTC-P3-415) T

WC5.4C $L$. ambigua SA

WC5.4d $L$. ambigua SA

WC5.4e $L$. ambigua SA

WC21.1i L. pauciflora SA

WC21.1b $L$. pauciflora SA

WC21.1j $L$. pauciflora SA

WC26.1d $L$. meyerianna SA

WC26.1e $L$. meyerianna SA

WC26.1f $L$. meyerianna SA

$P$. lemoignei (LMG2207)

$R$. detusculanense (APF11)

R. solanacearum (R221)

R. pickettii (ATCC27512)

Az. Caulinodans (LMG6465)

$P$. aeruginosa (ATCC10145)

K. pneumoniae (AU45)

B. andropogonis (ATCC23061) T

B. fungorum (LMG19076) T

B. caribiensi (MWAP64) T

B. caryophylii (ATCC25418) T

B. cepacia (ATCC25416) T

B. cocovenenas (ATCC33664) T gcacacaggt gctgcatggc tgtcgtc-ag ctcgtgtcgt gagatgttgg agacacaggt gctgcatggc tgtcgtc-ag ctcgtgtcgt gagatgttgg tgagacaggt gctgcatggc tgtcgtc-ag ctcgtgttgt gaaatgttgg

1060

1070

1080

1090

1100

gttaagtccc gcaacgagcg caaccettgt ccttagttgc ta------cg gttaagtccc gcaacgagcg caaccettgt ccctagttgc ta------cg gttaagtccc gcaacgagcg caacccttgt ccctagttcg ta------cg gttaagtccc gcaacgagcg caacccttgt ccttagttgc ta------cg gttaagtccc gcaacgagcg caacccttgt ccttagttgc ta------cg gttaagtccc gcaacgagcg caaccettgt ccttag---- -a------cg gttaagtccc gcaacgagcg caacccttgt ccttagttgc ta------cg gttaagtccc gcaacgagcg caaccettgt ccctagttgc ta------cg gttaagtccc gcaacgagcg caacccttgt ccttagttgc ta------cg gttaagtccc gcaacgagcg caacccttgt ccttagttgc ta------cg gttaagtccc gcaacgagcg caaccettgt ccttagttcg ta------cg gttaagtccc gcaacgagcg caacccttgt cctta---g- -a------cg gttaagtccc gcaacgagcg caacccttgt ccttagttgc ta------cg gttaagtccc gcaacgagcg caacccttgt ccctagttgc ta------cg gttaagtccc gcaacgagcg caacccttgt ccctagttgc ta------cg gttaagtccc gcaacgagcg caacccttgt ccttagttgc ta------cg gttaagtccc gcaacgagcg caacccttgt ccttagttgc ta------cg gttaagtccc gcaacgagcg caacccttgt ccttagttgc ta------cg gttaagtccc gcaacgagcg caaccettgt ccctagttgc ta------cg gttaagtccc gcaacgagcg caacccttgt ccctagttgc ta------cg gttaagtccc gcaacgagcg caacccttgt ccctagttgc ta------cg gttaagtccc gcaacgagcg caaccettgt ccctagttgc ta------cg gttaagtccc gcaacgagcg caacccttgt ctctagttgc ta------cg gttaagtccc gcaacgagcg caaccettgt ccttagttgc ta------cg gttaagtccc gcaacgagcg caaccettgt ccttagttgc ta------cg gttaagtccc gcaacgagcg caaccettgt cctta----- -a------cg gttaagtccc gcaacgagcg caaccettgt ccccagttgc ta------cg gttaagtccc gcaacgagcg caacccttgt ccctagttgc ta------cg gttaagtccc gcaacgagcg caacccttgt ctctagttgc ta------cg gttaagtccc gcaacgagcg caaccettgt ccttagttgc ta------cg gttaagtccc gcaacgagcg caacccttgt ccttagttgc ta------cg gttaagtccc gcaacgagcg caacccttgt ccctagttgc ta------cg gttaagtccc gcaacgagcg caacccttgt ccctagttgc ta------cg gttaagtccc gcaacgagcg caacccttgt ccttagttgc ta------co gttaagtccc gcaacgagcg caaccettgt ccctagttgc ta------cg gttaagtccc gcaacgagcg caaccettgt ccctagttgc ta------cg gttaagtccc gcaacgagcg caacccttgt ccctagttgc ta------cg gttaagtccc gcaacgagcg caacccttgt ccttagttgc ta------cg gttaagtccc gcaacgagcg caacccttgt ccttagttgc ta------cg gttaagtccc gcaacgagcg caacccttgt ccttagttgc ta------cg gttaagtccc gcaacgagcg caacccttgt ccttagttgc ta------cg gttaagtccc gcaacgagcg caacccttgt ccttagttgc ta------cg gttaagtccc gcaacgagcg caccccttgt ccctagttgc ta------cg gttaagtccc gcaacgagcg caaccettgt ccttagttgc ta------cg gttaagtccc gcaacgagcg caacccttgt ccctagttgc ta------cg gttaagtccc gcaacgagcg caacccttgt ccttagttgc ta------cg gttaagtccc gcaacgagcg caacccttgt ccttagttgc ta------cg gttaagtccc gcaacgagcg caacccttgt ccttagttgc ta------cg gttaagtccc gcaacgagcg caacccttgt ccctagttgc ta------cg gttaagtccc gcaacgagcg caacccttgt ccctagttgc ta------cg gttaagtccc gcaacgagcg caacccttgt ccctagttgc ta------cg gttaagtccc gcaacgagcg caacccttgt ccctagttgc ta------cg gttaagtccc gcaacgagcg caacccttgt ccctagttgc ta------cg gttaagtccc gcaacgagcg caacccttgt ccctagttgc ta------cg gttaagtccc gcaacgagcg caaccettgt ccctagttgc ta------cg gttaagtccc gcaacgagcg caacccttgt ccctagttgc ta------cg gttaagtccc gcaacgagcg caacccttgt ccctagttgc ta------cg gttaagtccc gcaacgagcg caacccttgt cattagttgc ta------cg gttaagtccc gcaacgagcg caacccttgt ctctagttgc ta------cg gttaagtccc gcaacgagcg caacccttgt ctctagttgc ta------cg gttaagtccc gcaacgagcg caacccttgt ctctagttgc ta------cg gttaagtccc gcaacgagcg caaccctcgc ctttagttgc catcatt-ca gttaagtccc gtaacgagcg caaccettgt ccttagttac cagcacctcg gttaagtccc gcaacgagcg caacccttat cctttgttgc cagcggttag

$$
1110
$$

1120

1130

1140

1150

caagggcact ctaaggagac tgccggtgac aaaccg-gag gaaggtgggg caagagcact ccagggagac tgccggtgac aaaccg-gag gaaggtgggg caagagcact ctagggagac tgccggtgac aaaccg-gag gaaggtgggg caagagcact ctaaggagac tgccggtgac aaaccg-gag gaaggtgggg caagagcact ctaaggagac tgccggtgac aaaccg-gag gaaggtgggg caagagcact ctagggagac tgccggtgac aaaccg-gag gaaggtgggg 
B. cocovenenas (LMG11626) T

$B$. fungorum (LMG16225)

B. gladioli (ATCC10248) T

B. glathei (LMG14190)T

B. glathei (ATCC29195) T

B. glathei (ATCC29195)T

B. glumae (LMG2196) T

B. graminis (C4D1M) T

B. hospita (LMG20598) T

B. mallei (ATCC23344)T

B. multivorans (LMG13010) T

B. norimbergensis (Y09879) T

B. phenazinium (LMG2247) T

B. phenoliruptrix (AC1100) T

B. phymatum (STM815) T

B. phytofirmans (PsJN) T

B. pickettii (ATCC27511)T

B. pseudomallei (ATCC23343) T

B. pyrrocinia (LMG14191) T

B. pyrrocinia ATCC15958)T

B. sacchari (AF263278) T

B. silvatlantica (SRMrh-20) T

B. solanacearum (ATCC11696) T

B. sordicola (S5-B) T

B. stabilis (LMG14294) T

B. terrae (KMY02) T

B. terriccola (LMG20594) T

B. thailandensis (E264) T

B. tropicalis (Ppe8) T

B. tuberum (STM678) T

B. unamae (MT1-641) T

B. vandii (LMG16020) T

B. vietnamiensis (AMMD) T

B. vietnamiensis (TVV75) T

B. vietnamiensis (TVV70) T

B. plantarii (LMG9035) T

B. xenovorans (LB400) T

B. cenocepacia (KN9) T

B. ferrariae (FeG101) T

B. ambifaria (LMG19182) T

B. oklahomensis (C6786) T

B. ubonensis (GTC-P3-415) T

WC5.4C $L$. ambigua SA

WC5.4d $L$. ambigua SA

WC5.4e L. ambigua SA

WC21.1i $L$. pauciflora SA

WC21.1b L. pauciflora SA

WC21.1j L. pauciflora SA

WC26.1d L. meyerianna SA

WC26.1e L. meyerianna SA

WC26.1f $L$. meyerianna SA

P. lemoignei (LMG2207)

$R$. detusculanense (APF11)

$R$. solanacearum (R221)

R. pickettii (ATCC27512)

Az. Caulinodans (LMG6465)

$P$. aeruginosa (ATCC10145)

$K$. pneumoniae (AU45)

B. andropogonis (ATCC23061) T

$B$. fungorum (LMG19076) T

B. caribiensi (MWAP64) T

B. caryophylii (ATCC25418) T

B. cepacia (ATCC25416) T

B. cocovenenas (ATCC33664) T

B. cocovenenas (LMG11626) T

$B$. fungorum (LMG16225)

B. gladioli (ATCC10248)T

B. glathei (LMG14190) T

B. glathei (ATCC29195) T

B. glathei (ATCC29195) T

B. glumae (LMG2196) T

B. graminis (C4D1M) T

B. hospita (LMG20598) T

B. mallei (ATCC23344)T

B. multivorans (LMG13010) T caagagcact ctagggagac tgccggtgac aaaccg-gag gaaggtgggg caagagcact ctagggagac tgccggtgac aaaccg-gag gaaggtgggg caagagcact ctagggagac tgccggtgac aaaccg-gag gaaggtgggg caagagcact ctaaggagac tgccggtgac aaaccg-gag gaaggtgggo caagagcact ctaaggagac tgccggtgac aaaccg-gag gaaggtgggg caagagcact ctaaggagac tgccggtgac aaaccg-gag gaaggtgggg caagagcact ctaaggagac tgccggtgac aaaccg-gag gaaggtgggg caagagcact ccagggagac tgccggtgac aaaccg-gag gaaggtgggg caagagcact ctagggagac tgccggtgac aaaccg-gag gaaggtgggg caagagcact ctaaggagac tgccggtgac aaaccg-gag gaaggtgggg caagagcact ctaaggagac tgccggtgac aaaccg-gag gaaggtgggg caagagcact ctaaggagac tgccggtgac aaacc--gag gaaggtgggg caagagcact ctagggagac tgccggtgac aaaccg-gag gaaggtgggg caagagcact ccagggagac tgccggtgac aaaccg-gag gaaggtgggg caagagcact ctagggagac tgccggtgac aaaccg-gag gaaggtgggg caagagcact ctagggagac tgccggtgac aaaccg-gag gaaggtgggg aaagggcact ctagagagac tgccggtgac aaaccg-gag gaaggtgggg caagagcact ctaaggagac tgccggtgac aaaccg-gag gaaggtgggg caagagcact ctaaggagac tgccggtgac aaaccg-gag gaaggtgggg caagagcact ctaaggagac tgccggtgac aaaccg-gag gaaggtgggg caagagcact ccggggagac tgccggtgac aaaccg-gag gaaggtgggg caagagcact ccagggagac tgccggtgac aaaccg-gag gaaggtgggg aaagggcact ctagagagac tgccggtgac aaaccg-gag gaaggtgggo caagagcact ctaaggagac tgccggtgac aaaccg-gag gaaggtgggg caagagcact ctaaggagac tgccggtgac aaaccg-gag gaaggtgggg caagagcact ctagggagac tgccggtgac aaaccg-gag gaaggtgggg caagagcact ccagggagac tgccggtgac aaaccg-gag gaaggtgggg caagagcact ctaaggagac tgccggtgac aaaccg-gaa gaaggtgggg caagagcact ccagggagac tgccggtgac aaaccg-gag gaaggtgggg caagagcact ccagggagac tgccggtgac aaaccg-gag gaaggtgggg caagagcact ccagggagac tgccggtgac aaaccg-gag gaaggtgggo caagagcact ctaaggagac tgccggtgac aaaccg-gag gaaggtgggg caagagcact ctaaggagac tgccggtgac aaaccg-gag gaaggtgggg caagagcact ctaaggagac tgccggtgac aaaccg-gag gaaggtgggo caagagcact ctaaggagac tgccggtgac aaaccg-gag gaaggtgggg caagagcact ctaaggagac tgccggtgac aaaccg-gag gaaggtgggg caagagcact ccagggagac tgccggtgac aacccg-gag gaaggtgggo caagagcact ctaaggagac tgccggtgac aaaccg-gag gaaggtgggg caagagcact ccagggagac tgccggtgac aaaccg-gag gaaggtgggg caagagcact ctaaggagac tgccggtgac aaaccg-gag gaaggtgggg caagagcact ctaaggagac tgccggtgac aaaccg-gag gaaggtgggg caagagcact ctaaggagac tgccggtgac aaaccg-gag gaaggtgggg caagagcact ccagggagac tgccggtgac aaaccg-gag gaaggtgggg caagagcact ccagggagac tgccggtgac aaaccg-gag gaaggtgggg caagagcact ccagggagac tgccggtgac aaaccg-gag gaaggtgggo caagagcact ccagggagac tgccggtgac aaaccg-gag gaaggtgggg caagagcact ccagggagac tgccggtgac aaaccg-gag gaaggtgggg caagagcact ccagggagac tgccggtgac aaaccg-gag gaaggtgggg caagagcact ccagggagac tgccggtgac aaaccg-gag gaaggtgggg caagagcact ccagggagac tgccggtgac aaaccg-gag gaaggtgggg caagagcact ccagggagac tgccggtgac aaaccg-gag gaaggtgggg aaagggcact ctaatgagac tgccggtgac aaaccg-gag gaaggtgggg aaagggcact ctagagagac tgccggtgac aaaccg-gag gaaggtgggo aaagggcact ctagagagac tgccggtgac aaaccg-gag gaaggtgggg aaagggcact ctagagagac tgccggtgac aaaccg-gag gaaggtgggg gttgggcact ctaaagggac tgccggtgat aagccgcgag gaaggtgggo ggtgggcact ctaaggagac tgccggtgac aaaccg-gag gaaggtgggg gccgggaact caaaggagac tgccagtgat aaactg-gag gaaggtgggg

1160 1170

1180

1190

1200

atgacgtcaa gtcctcatgg cccttatggg tagggcttca cacgtcatac atgacgtcaa gtcctcatgg cccttatggg tagggcttca cacgtcatac atgacgtcaa gtcctcatgg cccttatggg tagggcttca cacgtcatac atgacgtcaa gtcctcatgg cccttatggg tagggcttca cacgtcatac atgacgtcaa gtcctcatgg cccttatggg tagggcttca cacgtcatac atgacgtcaa gtcctcatgg cccttatggg tagggcttca cacgtcatac atgacgtcaa gtcctcatgg cccttatggg tagggcttca cacgtcatac atgacgtcaa gtcctcatgg cccttatggg tagggcttca cacgtcatac atgacgtcaa gtcctcatgg cccttatggg tagggcttca cacgtcatac atgacgtcaa gtcctcatgg cccttatggg tagggcttca cacgtcatac atgacgtcaa gtcctcatgg cccttatggg tagggcttca cacgtcatac atgacgtcaa gtcctcatgg cccttatggg tagggcttca cacgtcatac atgacgtcaa gtcctcatgg cccttatggg tagggcttca cacgtcatac atgacgtcaa gtcctcatgg cccttatggg tagggcttca cacgtcatac atgacgtcaa gtcctcatgg cccttatggg tagggcttca cacgtcatac atgacgtcaa gtcctcatgg cccttatggg tagggcttca cacgtcatac atgacgtcaa gtcctcatgg cccttatggg tagggcttca cacgtcatac 
B. norimbergensis (Y09879) T

B. phenazinium (LMG2247) T

B. phenoliruptrix (AC1100) T

B. phymatum (STM815) T

B. phytofirmans (PsJN) T

B. pickettii (ATCC27511)T

B. pseudomallei (ATCC23343) T

B. pyrrocinia (LMG14191) T

B. pyrrocinia ATCC15958)T

$B$. sacchari (AF263278)T

B. silvatlantica (SRMrh-20) T

B. solanacearum (ATCC11696) T

B. sordicola (S5-B) T

B. stabilis (LMG14294) T

B. terrae (KMY02) T

B. terriccola (LMG20594) T

B. thailandensis (E264)T

B. tropicalis (Ppe8) T

B. tuberum (STM678) T

B. unamae (MT1-641) T

B. vandi (LMG16020)T

B. vietnamiensis (AMMD) $T$

B. vietnamiensis (TVV75) T

B. vietnamiensis (TVV70) T

B. plantarii (LMG9035) T

B. xenovorans (LB400) T

B. cenocepacia (KN9) T

B. ferrariae (FeG101) T

B. ambifaria (LMG19182) T

B. oklahomensis (C6786) T

$B$. ubonensis (GTC-P3-415) T

WC5.4C L. ambigua SA

WC5.4d $L$. ambigua SA

WC5.4e $L$. ambigua SA

WC21.1i $L$. pauciflora SA

WC21.1b L. pauciflora SA

WC21.1j $L$. pauciflora SA

WC26.1d L. meyerianna SA

WC26.1e L. meyerianna SA

WC26.1f L. meyerianna SA

P. lemoignei (LMG2207)

$R$. detusculanense (APF11)

$R$. solanacearum (R221)

R. pickettii (ATCC27512)

Az. caulinodans (LMG6465)

$P$. aeruginosa (ATCC10145)

$K$. pneumoniae (AU45)

B. andropogonis (ATCC23061) T

$B$. fungorum (LMG19076) T

B. caribiensi (MWAP64) T

B. caryophylii (ATCC25418) T

B. cepacia (ATCC25416) T

B. cocovenenas (ATCC33664) T

B. cocovenenas (LMG11626) T

$B$. fungorum (LMG16225)

B. gladioli (ATCC10248) T

B. glathei (LMG14190)T

B. glathei (ATCC29195) T

B. glathei (ATCC29195)T

B. glumae (LMG2196) T

B. graminis (C4D1M) T

B. hospita (LMG20598) T

B. mallei (ATCC23344)T

B. multivorans (LMG13010) T

$B$. norimbergensis (Y09879) T

B. phenazinium (LMG2247) T

B. phenoliruptrix (AC1100) T

B. phymatum (STM815) T

B. phytofirmans (PsJN) T

B. pickettii (ATCC27511)T

B. pseudomallei (ATCC23343) T

B. pyrrocinia (LMG14191) T

B. pyrrocinia ATCC15958) T

B. sacchari (AF263278) T

B. silvatlantica (SRMrh-20) T atgacgtcaa gtcctcatgg cccttatggg tagggcttca cacgtcatac atgacgtcaa gtcctcatgg cccttatggg tagggcttca cacgtcatac atgacgtcaa gtcctcatgg cccttatggg tagggcttca cacgtcatac atgacgtcaa gtcctcatgg cccttatggg tagggcttca cacgtcatac atgacgtcaa gtcctcatgg cccttatggg tagggcttca cacgtcatac atgacgtcaa gtcctcatgg cccttatggg tagggcttca cacgtcatac atgacgtcaa gtcctcatgg cccttatggg tagggcttca cacgtcatac atgacgtcaa gtcctcatgg cccttatggg tagggcttca cacgtcatac atgacgtcaa gtcctcatgg cccttatggg tagggcttca cacgtcatac atgacgtcaa gtcctcatgg cccttatggg tagggcttca cacgtcatac atgacgtcaa gtcctcatgg cccttatggg tagggcttca cacgtcatac atgacgtcaa gtcctcatgg cccttatggg tagggcttca cacgtcatac atgacgtcaa gtcctcatgg cccttatggg tagggcttca cacgtcatac atgacgtcaa gtcctcatgg cccttatggg tagggcttca cacgtcatac atgacgtcaa gtcctcatgg cccttatggg tagggcttca cacgtcatac atgacgtcaa gtcctcatgg cccttatggg tagggcttca cacgtcatac atgacgtcaa gtcctcatgg cccttatggg tagggcttca cacgtcatac atgacgtcaa gtcctcatgg cccttatggg tagggcttca cacgtcatac atgacgtcaa gtcctcatgg cccttatggg tagggcttca cacgtcatac atgacgtcaa gtcctcatgg cccttatggg tagggcttca cacgtcatac atgacgtcaa gtcctcatgg cccttatggg tagggcttca cacgtcatac atgacgtcaa gtcctcatgg cccttatggg tagggcttca cacgtcatac atgacgtcaa gtcctcatgg cccttatggg tagggcttca cacgtcatac atgacgtcaa gtcctcatgg cccttatggg tagggcttca cacgtcatac atgacgtcaa gtcctcatgg cccttatggg tagggcttca cacgtcatac atgacgtcaa gtcctcatgg cccttatggg tagggcttca cacgtcatac atgacgtcaa gtcctcatgg cccttatggg tagggcttca cacgtcatac atgacgtcaa gtcctcatgg cccttatggg tagggcttca cacgtcatac atgacgtcaa gtcctcatgg cccttatggg tagggcttca cacgtcatac atgacgtcaa gtcctcatgg cccttatggg tagggcttca cacgtcatac atgacgtcaa gtcctcatgg cccttatggg tagggcttca cacgtcatac atgacgtcaa gtcctcatgg cccttatggg tagggcttca cacgtcatac atgacgtcaa gtcctcatgg cccttatggg tagggcttca cacgtcatac atgacgtcaa gtcctcatgg cccttatggg tagggcttca cacgtcatac atgacgtcaa gtcctcatgg cccttatggg tagggcttca cacgtcatac atgacgtcaa gtcctcatgg cccttatggg tagggcttca cacgtcatac atgacgtcaa gtcctcatgg cccttatggg tagggcttca cacgtcatac atgacgtcaa gtcctcatgg cccttatggg tagggcttca cacgtcatac atgacgtcaa gtcctcatgg cccttatggg tagggcttca cacgtcatac atgacgtcaa gtcctcatgg cccttatggg tagggcttca cacgtcatac atgacgtcaa gtcctcatgg cccttatggg tagggcttca cacgtcatac atgacgtcaa gtcctcatgg cccttatggg tagggcttca cacgtcatac atgacgtcaa gtcctcatgg cccttatggg tagggcttca cacgtcatac atgacgtcaa gtcctcatgg cccttatggg tagggcttca cacgtcatac atgacgtcaa gtcctcatgg cccttacggg ctgggctaca cacgtgctac atgacgtcaa gtcatcatgg cccttacggc cagggctaca cacgtgctac atgacgtcaa gtcatcatgg cccttacgac cagggctaca cacgtgctac

1210

1220

1230

1240

1250

aatggtcgga acagagggct gccaacccgc gagggggagc taatcccaga aatggtcgga acagagggtc gccaacccgc gagggggagc caatcccaga aatggtcgga acagagggtt gccaagccgc gaggtggagc caatcccaga aatggtcgga acagagggtc gccaacccgc gagggggagc caatcccaga aatggtcgga acagagggtt gccaacccgc gagggggagc taatcccaga aatggtcgga acagagggtc gccaacccgc gagggggagc taatcccaga aatggtcgga acagagggtc gccaacccgc gagggggagc taatcccaga aatggtcgga acagagggtc gccaacccgc gagggggagc caatcccaga aatggtcgga acagagggtc gccaacccgc gagggggagc taatcccaga aatggtcgga acagagggtc gctaagccgc gaggtggagc caatcccaga aatggtcgga acagagggtc gctaagccgc gaggtggagc caatcccaga aatggtcgga acagagggtc gctaagccgc gaggtggagc caatcccaga aatggtcgga acagagggtt gccaacccgc gagggggagc taatcccaga aatggtcgga acagagggtc gccaagccgc gaggtggagc caatcccaga aatggtcgga ayagagggtt gccaagccgc gaggtggagc caatcccaga aatggtcgga acagagggtc gccaacccgc gagggggagc caatcccaga aatggtcgga acagagggtt gccaacccgc gagggggagc taatcccaga aatggtcggt acagagggct gccaaatcgc gagatggagc taaccccaga aatggtcgga acagagggtc gccaacccgc gagggggagc caatcccaga aatggtcgga acagagggtc gccaagccgc gaggtggagc caatcccaga aatggtcgga acagagggtt gccaagccgc gaggcggagc caatcccaga aatggtcgga acagagggtc gccaacccgc gagggggagc caatcccaga aatggtgcat acagagggtt gccaagccgc gaggtggagc taatcccaga aatggtcgga acagagggtc gccaacccgc gagggggagc caatcccaga aatggtcgga acagagggtt gccaacccgc gagggggagc taatcccaga aatggtcgga acagagggtt gccaacccgc gagggggagc taatcccaga aatggtcgga acagagggtt gcgaagccgc gaggtggagc caatcccaga aatggtcgga acagagggta gccaagccgc gaggtggagc caatcccgga 
B. solanacearum (ATCC11696) T

B. sordicola (S5-B) T

B. stabilis (LMG14294)T

$B$. terrae (KMY02) T

B. terriccola (LMG20594) T

$B$. thailandensis (E264) T

B. tropicalis (Ppe8) T

B. tuberum (STM678) T

B. unamae (MT1-641) T

B. vandii (LMG16020) T

$B$. vietnamiensis (AMMD) T

B. vietnamiensis (TVV75) T

B. vietnamiensis (TVV70)T

B. plantarii (LMG9035) T

B. xenovorans (LB400) T

B. cenocepacia (KN9) T

$B$. ferrariae (FeG101) T

B. ambifaria (LMG19182) T

B. oklahomensis (C6786) T

B. ubonensis (GTC-P3-415) T

WC5.4C $L$. ambigua SA

WC5.4d $L$. ambigua SA

WC5.4e $L$. ambigua SA

WC21.1i $L$. pauciflora SA

WC21.1b $L$. pauciflora SA

WC21.1j $L$. pauciflora SA

WC26.1d $L$. meyerianna SA

WC26.1e $L$. meyerianna SA

WC26.1f $L$. meyerianna SA

$P$. lemoignei (LMG2207)

$R$. detusculanense (APF11)

$R$. solanacearum (R221)

R. pickettii (ATCC27512)

Az. caulinodans (LMG6465)

$P$. aeruginosa (ATCC10145)

$K$. pneumoniae (AU45)

B. andropogonis (ATCC23061) T

$B$. fungorum (LMG19076) T

B. caribiensi (MWAP64) T

B. caryophylii (ATCC25418) T

B. cepacia (ATCC25416) T

B. cocovenenas (ATCC33664)T

B. cocovenenas (LMG11626) T

$B$. fungorum (LMG16225)

B. gladioli (ATCC10248) T

B. glathei (LMG14190) T

B. glathei (ATCC29195)T

B. glathei (ATCC29195) T

B. glumae (LMG2196) T

B. graminis (C4D1M) T

B. hospita (LMG20598) T

B. mallei (ATCC23344) T

B. multivorans (LMG13010) T

$B$. norimbergensis (Y09879) T

B. phenazinium (LMG2247) T

B. phenoliruptrix (AC1100) T

B. phymatum (STM815) T

B. phytofirmans (PsJN) T

B. pickettii (ATCC27511)T

B. pseudomallei (ATCC23343) T

B. pyrrocinia (LMG14191) T

B. pyrrocinia ATCC15958) T

B. sacchari (AF263278) T

$B$. silvatlantica (SRMrh-20)T

B. solanacearum (ATCC11696) T

B. sordicola (S5-B) T

B. stabilis (LMG14294) T

B. terrae (KMY02) T

B. terriccola (LMG20594) T

B. thailandensis (E264) T

$B$. tropicalis (Ppe8) T

B. tuberum (STM678) T

B. unamae (MT1-641) T

B. vandii (LMG16020) T

$B$. vietnamiensis (AMMD) T aatggtgcat acagagggtt gccaagccgc gaggtggagc taatcccaga aatggtcgga acagagggct gccaacccgt gagggggagc taatcccaga aatggtcgga acagagggtt gccaacccgc gagggggagc taatcccaga aatggtcgga acagagggtt gccaagccgc gaggtggagc calcccaga aatggtcgga acagagggtc gccaagccgc gaggtggagc caatcccaga aatggtcgga acagagggtc gccaacccgc gagggggagc caatcccaga aatggtcgga acagagggtt gccaagccgc gaggtggagc caatcccaga aatggtcgga acagagggtt gccaagccgc gaggtggagc caatcccaga aatggtcgga acagagggtt gccaagccgc gaggtggagc caatcccaga aatggtcgga acagagggtc gccaacccgc gagggggagc taatcccaga aatggtcgga acagagggtt gccaacccgc gagggggagc taatcccaga aatggtcgga acagagggtt gccaacccgc gagggggagc taatcccaga aatggtcgga acagagggtt gccaacccgc gagggggagc taatcccaga aatggtcgga acagagggtc gccaacccgc gagggggagc taatcccaga aatggtcgga acagagggtc gccaacccgc gagggggagc calcccaga aatggtcgga acagagggtt gccaacccgc gagggggagc taatcccaga aatggtcgga acagagggtt gcgaagccgc gaggtggagc caatcccaga aatggtcgga acagagggtt gccaacccgc gagggggagc taatcccaga aatggtcgga acagagggtc gccaacccgc gagggggagc caatcccaga aatggtcgga acagagggtc gccaacccgc gagggggagc taatcccaga aatggtcgga acagagggtt gccaagccgc gaggtggagc caatcccaga aatggtcgga acagagggtc gccaacccgc gagggggagc caatcccaga aatggtcgga acagagggtc gccaacccgc gagggggagc caatcccaga aatggtcgga acagagggtt gccaagccgc gaggtggagc caatcccgga aatggtcgga acagagggtt gccaagccgc gaggtggagc caatcccgga aatggtcgga acagagggtt gccaagccgc gaggtggagc caltcccaga aatggtcgga acagagggtt gccaagccgc gaggtggagc caatcccaga aatggtcgga acagagggtt gccaagccgc gaggtggagc caatcccaga aatggtcgga acagagggtt gccaagccgc gaggtggagc caatcccaga aatggtacat acagagggct gccaacccgc gagggggagc taatcccaga aatggtgcat acagagggtt gccaagccgc gaggtggagc taatcccaga aatggtgcat acagagggtt gccaagccgc gaggtggagc taatcccaga aatggtgcat acagagggtt gccaagccgc gaggtggagc taatcccaga aatggcggtg acaatgggat gcgagcctgc gagggtgagc aaatctccaa aatggtcggt acaaagggtt gccaagccgc gaggtggagc taatcccata aatggcatat acaaagagaa gcgacctcgc gagagcaagc ggacctcata$$
1260
$$

1270

1280

1290

1300

aaccgat-c gtagt-ccgg attgcactct gcaactcgag tgcatgaagt aaaccgat-c gtagt-ccgg atcgcactct gcaactcgag tgcgtgaagc aaaccgat-c gtagt-ccgg atcgcagtct gcaactcgac tgcgtgaagc aaaccgat-c gtagt-ccgg attgcactct gcaactcgag tgcatgaagc aaaccgat-c gtagt-ccgg attgcactct gcaactcgag tgcatgaagc aaaccgat-c gtagt-ccgg attgcactct gcaactcgag tgcatgaagc aaccgat-c gtagt-ccgg attgcactct gcaactcgag tgcatgaagc aaaccgat-c gtagt-ccgg atcgcactct gcaactcgag tgcgtgaagc aaaccgat-c gtagt-ccgg attgcactct gcaactcgag tgcatgaagc aaccgat-c gtagt-ccgg atcgtagtct gcaactcgac tacgtgaagc aaaccgat-c gtagt-ccgg atcgtagtct gcaactcgac tacgtgaagc aaaccgat-c gtagt-ccgg atcgtagtct gcaactcgac tacgtgaagc aaaccgat-c gtagt-ccgg attgcactct gcaactcgag tgcatgaagc aaaccgat-c gtagt-ccgg atcgcactct gcaactcgag tgcgtgaagc aalccgat-c gtagt-ccgg atcgcagtct gcaactcgac tgcgtgaagc aaaccgat-c gtagt-ccgg attgcactct gcaactcgag tgcatgaagc aaaccgat-c gtagt-ccgg attgcactct gcaactcgag tgcatgaagc aaaccgat-c gtagt-ccgg atcgcagtct gcaactcgac tgcgtgaagt aaaccgat-c gtagt-ccgg atcgcactct gcaactcgag tgcgtgaagc aaaccgat-c gtagt-ccgg atcgcactct gcaactcgag tgcgtgaagc aaaccgat-c gtagt-ccgg atcgcagtct gcaactcgac tgcgtgaagc aaaccgat-c gtagt-ccgg atcgcactct gcaactcgag tgcgtgaagc aatgcat-c gtagt-ccgg atcgtagtct gcaactcgac tacgtgaagc aaaccgat-c gtagt-ccgg attgcactct gcaactcgag tgcatgaagc aaaccgat-c gtagt-ccgg attgcactct gcaactcgag tgcatgaagc aaaccgat-c gtagt-ccgg attgcactct gcaactcgag tgcatgaagc aaaccgat-c gtagt-ccgg atcgcagtct gcaactcgac tgcgtgaagc aaaccgat-c gtagt-ccgg atcgcagtct gcaactcgac tgcgtgaagc aatgcat-c gtagt-ccgg atcgtagtct gcaactcgac tacgtgaagc aaaccgat-c gtagt-ccgg atcgtagtct gcaactcgac tacgtgaagc aaaccgat-c gtagt-ccgg attgcactct gcaactcgag tgcatgaagc aaaccgat-c gtagt-ccgg atcgcagtct gcaactcgac tgcgtgaagc aaaccgat-c gtagt-ccgg atcgcactct gcaactcgag tgcgtgaagc aaaccgat-c gtagt-ccgg attgcactct gcaactcgag tgcatgaagc aaaccgat-c gtagt-ccgg atcgcagtct gcaactcgac tgcgtgaagc aaaccgat-c gtagt-ccgg atcgcactct gcaactcggg tgcgtgaagc aaaccgat-c gtagt-ccgg atcgcagtct gcaactcgac tgcgtgaagc aaaccgat-c gtagt-ccgg attgcactct gcaactcgag tgcatgaagc aaaccgat-c gtagt-ccgg attgcactct gcaactcgag tgcatgaagc 
B. vietnamiensis (TVV75)T

B. vietnamiensis (TVV70)T

B. plantarii (LMG9035) T

B. xenovorans (LB400) T

$B$. cenocepacia (KN9) T

$B$. ferrariae (FeG101) T

B. ambifaria (LMG19182) T

$B$. oklahomensis (C6786) T

B. ubonensis (GTC-P3-415) T

WC5.4C $L$. ambigua SA

WC5.4d $L$. ambigua SA

WC5.4e $L$. ambigua SA

WC21.1i $L$. pauciflora SA

WC21.1b $L$. pauciflora SA

WC21.1j $L$. pauciflora SA

WC26.1d $L$. meyerianna SA

WC26.1e $L$. meyerianna SA

WC26.1f $L$. meyerianna SA

$P$. lemoignei (LMG2207)

$R$. detusculanense (APF11)

$R$. solanacearum (R221)

R. pickettii (ATCC27512)

Az. caulinodans (LMG6465)

$P$. aeruginosa (ATCC10145)

$K$. pneumoniae (AU45)

B. andropogonis (ATCC23061) T

$B$. fungorum (LMG19076) $T$

B. caribiensi (MWAP64) T

B. caryophylii (ATCC25418) T

B. cepacia (ATCC25416) T

B. cocovenenas (ATCC33664)T

$B$. cocovenenas (LMG11626) T

$B$. fungorum (LMG16225)

B. gladioli (ATCC10248) T

B. glathei (LMG14190) T

B. glathei (ATCC29195) T

B. glathei (ATCC29195) T

B. glumae (LMG2196) T

$B$. graminis (C4D $1 \mathrm{M}) \mathrm{T}$

B. hospita (LMG20598) T

B. mallei (ATCC23344) T

B. multivorans (LMG13010) T

B. norimbergensis (Y09879) T

B. phenazinium (LMG2247)T

B. phenoliruptrix (AC1100) T

B. phymatum (STM815) T

B. phytofirmans (PsJN) T

B. pickettii (ATCC27511)T

B. pseudomallei (ATCC23343) T

B. pyrrocinia (LMG14191)T

B. pyrrocinia ATCC15958) T

B. sacchari (AF263278) T

B. silvatlantica (SRMrh-20)T

B. solanacearum (ATCC11696) T

$B$. sordicola (S5-B) $T$

B. stabilis (IMG14294) T

B. terrae (KMY02) T

B. terriccola (LMG20594) T

B. thailandensis (E264) T

$B$. tropicalis (Ppe8) T

B. tuberum (STM678) T

B. unamae (MT1-641) T

$B$. vandii (LMG16020) T

$B$. vietnamiensis (AMMD) T

B. vietnamiensis (TVV75) T

B. vietnamiensis (TVV70)T

B. plantarii (LMG9035) T

B. xenovorans (LB400) T

$B$. cenocepacia (KN9) T

B. ferrariae (FeG101) T

B. ambifaria (LMG19182) T

B. oklahomensis (C6786) T

B. ubonensis (GTC-P3-415) T

WC5.4C L. ambigua SA

WC5.4d L. ambigua SA aaccgat-c gtagt-ccgg attgcactct gcaactcgag tgcatgaagc aaccgat-c gtagt-ccgg attgcactct gcaactcgag tgcatgaagc aaccgat-c gtagt-ccgg attgcactct gcaactcgag tgcatgaagc aaaccgat-c gtagt-ccgg atcgcactct gcaactcggg tgcgtgaagc aaaccgatgc gtagtgccgg attgca-

aaccgat-c gtagt-ccgg atcgcagtct gcaactcgac tgcgtgaagc aaaccgat-c gtagt-ccgg attgcactct gcaactcgag tgcatgaagc aaaccgat-c gtagt-ccgg attgcactct gcaactcgag tgcatgaagc aaaccgat-c gtagt-ccgg attgcactct gcaactcgag tgcatgaagc aaaccgat-c gtagt-ccgg atcgcactct gcaactcggg tgcgtgaagc aaaccgat-c gtagt-ccgg atcgcactct gcaactcgag tgcgtgaagc aaaccgat-c gtagt-ccgg atcgcactct gcaactcgag tgcgtgaagc aaaccgat-c gtagt-ccgg atcgcactct gcaactcgag tgcgtgaagc aaaccgat-c gtggt-ccgg atcgcactct gcaactcgag tgcgtgaagc aaccgat-c gtagt-ccgg atcgcactct gcaactcggg tgcgtgaagc aaaccgat-c gtagt-ccgg atcgcactct gcaactcggg tgcgtgaagc aaaccgat-c gtagt-ccgg atcgcactct gcaactcggg tgcgtgaagc aaaccgat-c gtagt-ccgg atcgcactct gcaactcggg tgcgtgaagc aagtgtat-c gtagt-ccgg attgtagtct gcaactcgac tacatgaagt aatgcat-c gtagt-ccgg atcgtagtct gcaactcgac tacgtgaagc aatgcat-c gtagt-ccgg atcgtagtct gcaactcgac tacgtgaagc aaatgcat-c gtagt-ccgg atcgtagtct gcaactcgac tacgtgaagc aagccg-t-c tcagt-tcgg attgcactct gcaactcgag tgcatgaagt aaaccgat-c gtagt-ccgg atcgcagtct gcaactcgac tgcgtgaagt aagtatgt-c gtagt-ccgg attggagtct gcaactcgac tccatgaagt

1310

1320

1330

1340

1350

tggaatcget agtaatcocg gatcagcatg ccgcggtgaa ta-cgttccc tggaatcgct agtaatcgcg gatcagcatg ccgcggtgaa ta-cgttccc tggaatcgct agtaatcgcg gatcagcatg ccgcggtgaa ta-cgttccc tggaatcgct agtaatcgcg gatcagcatg ccgcggtgaa ta-cgttccc tggaatcgct agtaatcgcg gatcagcatg ccgcggtgaa ta-cgttccc tggaatcgct agtaatcgcg gatcagcatg ccgcggtgaa ta-cgttccc tggaatcgct agtaatcgcg gatcagcatg ccgcggtgaa ta-cgttccc tggaatcgct agtaatcgcg gatcagcatg ccgcggtgaa ta-cgttccc tggaatcgct agtaatcgcg gatcagcatg ccgcggtgaa ta-cgttccc tggaatcgct agtaatcgcg gatcagcatg ccgcggtgaa ta-cgttccc tggaatcgct agtaatcgcg gatcagcatg ccgcggtgaa ta-cgttccc tggaatcgct agtaatcgcg gatcagcatg ccgcggtgaa ta-cgttccc tggaatcgct agtaatcgcg gatcagcatg ccgcggtgaa ta-cgttccc tggaatcgct agtaatcgcg gatcagcatg ccgcggtgaa ta-cgttccc tggaatcgct agtaatcgcg gatcagcatg ccgcggtgaa taccgttccc tggaatcgct agtaatcgcg gatcagcatg ccgcggtgaa ta-cgttccc tggaatcgct agtaatcgcg gatcagcatg ccgcggtgaa ta-cgttccc tggaatcgct agtaatcgcg gatcagcatg tcgcggtgaa ta-cgttccc tggaatcgct agtaatcgcg gatcagcatg ccgcggtgaa ta-cgttccc tggaatcgct agtaatcgcg gatcagcatg ccgcggtgaa ta-cgttccc tggaatcgct agtaatcgcg gatcagcatg ccgcggtgaa ta-cgttccc tggaatcgct agtaatcgcg gatcagcatg ccgcggtgaa ta-cgttccc tggaatcgct agtaatcgcg gatcagcatg ccgcggtgaa ta-cgttccc tggaatcgct agtaatcgcg gatcagcatg ccgcggtgaa ta-cgttccc tggaatcgct agtaatcgcg gatcagcatg ccgcggtgaa ta-cgttccc tggaatcgct agtaatcgcg gatcagcatg ccgcggtgaa ta-cgttccc tggaatcgct agtaatcgcg gatcagcatg ccgcggtgaa ta-cgttccc tggaatcgct agtaatcgcg gatcagcatg ccgcggtgaa ta-cgttccc tggaatcgct agtaatcgcg gatcagcatg ccgcggtgaa ta-cgttccc tggaatcgct agtaatcgcg gatcagcatg ccgcggtgaa ta-cgttccc tggaatcgct agtaatcgcg gatcagcatg ccgcggtgaa ta-cgttccc tggaatcgct agtaatcgcg gatcagcatg ccgcggtgaa ta-cgttccc tggaatcgct agtaatcgcg gatcagcatg ccgcggtgaa ta-cgttccc tggaatcgct agtaatcgcg gatcagcatg ccgcggtgaa ta-cgttccc tggaatcgct agtaatcgcg gatcagcatg ccgcggtgaa ta-cgttccc tggaatcgct agtaatcgcg gatcagcatg ccgcggtgaa ta-cgttccc tggaatcgct agtaatcgcg gatcagcatg ccgcggtgaa ta-cgttccc tggaatcgct agtaatcgcg gatcagcatg ccgcggtgaa ta-cgttccc tggaatcgct agtaatcgcg gatcagcatg ccgcggtgaa ta-cgttccc tggaatcgct agtaatcgcg gatcagcatg ccgcggtgaa ta-cgttccc tggaatcgct agtaatcgcg gatcagcatg ccgcggtgaa ta-cgttccc tggaatcgct agtaatcgcg gatcagcatg ccgcggtgaa ta-cgttccc tggaatcgct agtaatcgcg gatcagcatg ccgcggtgaa ta-cgttccc

tggaatcgct agtaatcgcg gatcagcatg ccgcggtgaa ta-cgttccc tggaatcgct agtaatcgcg gatcagcatg ccgcggtgaa ta-cgttccc tggaatcgct agtaatcgcg gatcagcatg ccgcggtgaa ta-cgttccc tggaatcgct agtaatcgcg gatcagcatg ccgcggtgaa ta-cgttccc tggaatcgct agtaatcgcg gatcagcatg ccgcggtgaa ta-cgttccc tggaatcgct agtaatcgcg gatcagcatg ccgcggtgaa ta-cgttccc 
WC5.4e $L$. ambigua SA WC21.1i L. pauciflora SA WC21.1b $L$. pauciflora SA WC21.1j $L$. pauciflora SA WC26.1d $L$. meyerianna SA WC26.1e $L$. meyerianna SA WC26.1f $L$. meyerianna SA $P$. lemoignei (LMG2207)

$R$. detusculanense (APF11)

$R$. solanacearum (R221)

R. pickettii (ATCC27512)

Az. caulinodans (LMG6465)

$P$. aeruginosa (ATCC10145)

$K$. pneumoniae (AU45)

B. andropogonis (ATCC23061) T

$B$. fungorum (LMG19076) $T$

B. caribiensi (MWAP64) T

B. caryophylii (ATCC25418) T

B. cepacia (ATCC25416) T

B. cocovenenas (ATCC33664) T

$B$. cocovenenas (LMG11626) T

$B$. fungorum (LMG16225)

B. gladioli (ATCC10248) T

B. glathei (LMG14190) T

B. glathei (ATCC29195)T

B. glathei (ATCC29195) T

B. glumae (LMG2196) T

$B$. graminis (C4D1M) T

B. hospita (LMG20598) T

B. mallei (ATCC23344) T

B. multivorans (LMG13010) T

B. norimbergensis (Y09879) T

B. phenazinium (LMG2247) T

B. phenoliruptrix (AC1100) T

B. phymatum (STM815) T

B. phytofirmans (PsJN) T

B. pickettii (ATCC27511)T

B. pseudomallei (ATCC23343) T

B. pyrrocinia (LMG14191) T

B. pyrrocinia ATCC15958)T

B. sacchari (AF263278) T

B. silvatlantica (SRMrh-20) T

B. solanacearum (ATCC11696) T

B. sordicola (S5-B) T

B. stabilis (LMG14294) T

B. terrae (KMY02) T

B. terriccola (LMG20594) T

$B$. thailandensis (E264) T

B. tropicalis (Ppe8) T

B. tuberum (STM678) T

B. unamae (MT1-641) T

$B$. vandii (LMG16020) T

$B$. vietnamiensis (AMMD) T

B. vietnamiensis (TVV75) T

$B$. vietnamiensis (TVV70)T

B. plantarii (LMG9035) T

B. xenovorans (LB400) T

$B$. cenocepacia (KN9) T

$B$. ferrariae (FeG101) T

B. ambifaria (LMG19182) T

$B$. oklahomensis (C6786) T

B. ubonensis (GTC-P3-415) T

WC5.4C $L$. ambigua SA

WC5.4d $L$. ambigua SA

WC5.4e $L$. ambigua SA

WC21.1i L. pauciflora SA

WC21.1b $L$. pauciflora SA

WC21.1j $L$. pauciflora SA

WC26.1d $L$. meyerianna SA

WC26.1e $L$. meyerianna SA

WC26.1f $L$. meyerianna SA

$P$. lemoignei (LMG2207)

$R$. detusculanense (APF11)

R. solanacearum (R221)

R. pickettii (ATCC27512) tggaatcgct agtaatcgcg gatcagcatg ccgcggtgaa ta-cgttccc tggaatcgct agtaatcgcg gatcagcatg ccgcgg-aga ta-cgttccc tggaatcgct agtaatcgcg gatcagcatg ccgcat-gaa ta-cgttccc tggaatcgct agtaatcgcg gatcagcatg ccgcggtgaa ta-cgttccc tggaatcgct agtaatcgcg gatcagcatg cggcggtgaa ta-cgttccc tggaatcgct agtaatcgcg gatcagcatg ccgcggtgaa ta-cgttccc tggaatcgct agtaatcgcg gatcagcatg ccgcggtgaa ta-cgttccc tggaatcgct agtaatcgcg gatcagcatg tcgcggtgaa ta-cgttccc tggaatcgct agtaatcgcg gatcagcatg ccgcggtgaa ta-cgttccc tggaatcgct agtaatcgcg gatcagcatg ccgcggtgaa ta-cgttccc tggaatcgct agtaatcgcg gatcagcatg ccgcggt-

tggaatcgct agtaatcgtg gatcagcatg ccacggtgaa ta-cgttccc cggaatcgct agtaatcgtg aatcagaatg tcacggtgaa ta-cgttccc cggaatcgct agtaatcgta gatcagaatg ctacggtgaa ta-cgttccc

1360

1370

1380

1390

1400

gggtcttgta cacaccgccc gtcacaccat gggagcgggt ttcaccagaa gggtcttgta cacaccgccc gtcacaccat gggagtgggt ttcaccagaa gggtcttgta cacaccgccc gtcacaccat gggagtgggt tttaccagaa gggtcttgta cacaccgccc gtcacaccat gggagtgggt tttaccagaa gggtcttgta cacaccgccc gtcacaccat gggagtgggt tttaccagaa gggtcttgta cacaccgccc gtcacaccat gggagtgggt tttaccagaa gggt-ttgta cacaccgccc gtcacaccat gggagtgggt tttaccagaa gggtcttgta cacaccgccc gtcacaccat gggagtgggt tttaccagaa gggtcttgta cacaccgccc gtcacaccat gggagtgggt tttaccagaa gggtcttgta cacaccgccc gtcacaccat gggagtgggt tttaccagaa gggtcttgta cacaccgccc gtcacaccat gggagtgggt tttaccagaa gggtcttgta cacaccgccc gtcacaccat gggagtgggt tttaccagaa gggtcttgta cacaccgccc gtcacaccat gggagtgggt tttaccagaa gggtcttgta cacaccgccc gtcacaccat gggagtgggt tttaccagaa gggtcttgta cacaccgccc gtcacaccat gggagtgggt tttaccagaa gggtcttgta cacaccgccc gtcacaccat gggagtgggt tttaccagaa gggtcttgta cacaccgccc gtcacaccat gggagtgggt tttaccagaa gggtcttgta cacaccgccc gtcacaccat gggagtgggt tttgccagaa gggtcttgta cacaccgccc gtcacaccat gggagtgggt tttaccagaa gggtcttgta cacaccgccc gtcacaccat gggagtgggt tttaccagaa gggtcttgta cacaccgccc gtcacaccat gggagtgggt tttaccagaa gggtcttgta cacaccgccc gtcacaccat gggagtgggt tttaccagaa gggtcttgta cacaccgccc gtcacaccat gggagtgggc tttaccagaa gggtcttgta cacaccgccc gtcacaccat gggagtgggt tttaccagaa gggtcttgta cacaccgccc gtcacaccat gggagtgggt tttaccagaa gggtcttgta cacaccgccc gtcacaccat gggagtgggt tttaccagaa gggtcttgta cacaccgccc gtcacaccat gggagtgggt tttgccagaa gggtcttgta cacaccgccc gtcacaccat gggagtgggt tttgccagaa gggtcttgta cacaccgccc gtcacaccat gggagtgggt tttgccagaa gggtcttgta cacaccgccc gtcacaccat gggagtgggt tttaccagaa gggtcttgta cacaccgccc gtcacaccat gggagtgggt tttaccagaa gggtcttgta cacaccgccc gtcacaccat gggagtgggt tttaccagaa gggtcttgta cacaccgccc gtcacaccat gggagtgggt tttaccagaa gggtcttgta cacaccgccc gtcacaccat gggagtgggt tttaccagaa gggtcttgta cacaccgccc gtcacaccat gggagtgggt tttgccagaa gggtcttgta cacaccgccc gtcacaccat gggagtgggt tttaccagaa gggtcttgta cacaccgccc gtcacaccat gggagtgggt tttgccagaa gggtcttgta cacaccgccc gtcacaccat gggagtgggt tttaccagaa gggtcatgta cacaccgccc gtcacaccat gggagtgggt tttaccagaa gggtcttgta cacaccgccc gtcacaccat gggagtgggt tttaccagaa gggtcttgta cacaccgccc gtcacaccat gggagtgggt tttaccagaa gggtcttgta cacaccgccc gtcacaccat gggagtgggt tttaccagaa gggtcttgta cacaccgccc gtcacaccat gggagtgggt tttaccagaa

gggtcttgta cacaccgccc gtcacaccat gggagtgggt tttgccagaa gggtcttgta cacaccgccc gtcacaccat gggagtgggt tttaccagaa gggtcttgta cacaccgccc gtcacaccat gggagtgggt tttaccagaa gggtcttgta cacaccgccc gtcacaccat gggagtgggt tttaccagaa gggtcttgta cacaccgccc gtca-agcat gggagtgggt tttaccagaa gggtcttgta cacaccgccc gtcacaccat gggagtgggt ttcaccagaa gggtcttgta cacaccgccc gtcacaccat gggagtgggt ttcaccagaa gggtcttgta cacaccggcc gtt---cacg tggagtgggt tttaccagaa gggtcttgta cacaccgccc gtcactccat gggagtgggt tttaccagaa gggtcttgta cacaccgccc gtcacaccat gggagtgggt ttcaccagaa gggtcttgta cacaccgccc a-aagaccat gggagtgggt tttaccagaa gggtcttgta cacaccgccc gtctctccat gggagtgggt tttaccagaa gggtcttgta cacaccgccc gacacaccat gggagtgggt tttaccagaa gggtcttgta cacaccgccc gtcacaccat gggagcgggt tttaccagaa gggtcttgta cacaccgccc gtcacaccat gggagtgggc tttaccagaa gggtcttgta cacaccgccc gtcacaccat gggagtgggc tttaccagaa 
Az. Caulinodans (LMG6465)

$P$. aeruginosa (ATCC10145)

$K$. pneumoniae (AU45)

B. andropogonis (ATCC23061) T

$B$. fungorum (LMG19076) T

B. caribiensi (MWAP64)T

B. caryophylii (ATCC25418) T

B. cepacia (ATCC25416) T

B. cocovenenas (ATCC33664)T

B. cocovenenas (LMG11626) T

$B$. fungorum (LMG16225)

B. gladioli (ATCC10248) T

B. glathei (LMG14190) T

B. glathei (ATCC29195)T

B. glathei (ATCC29195)T

B. Glumae (LMG2196) T

B. graminis (C4D1M) T

B. hospita (LMG20598) T

B. mallei (ATCC23344) T

B. multivorans (LMG13010) T

$B$. norimbergensis (Y09879) T

B. phenazinium (LMG2247) T

B. phenoliruptrix (AC1100) T

B. phymatum (STM815) T

B. phytofirmans (PsJN) T

B. pickettii (ATCC27511) T

B. pseudomallei (ATCC23343) T

B. pyrrocinia (LMG14191)T

B. pyrrocinia ATCC15958) T

$B$. sacchari (AF263278) T

B. silvatlantica (SRMrh-20) T

B. solanacearum (ATCC11696)T

B. sordicola (S5-B) T

B. stabilis (LMG14294) T

B. terrae (KMY02) T

B. terriccola (LMG20594) T

$B$. thailandensis (E264) T

$B$. tropicalis (Ppe8) $\mathrm{T}$

B. tuberum (STM678) T

B. unamae (MT1-641) T

B. vandii (LMG16020) T

$B$. vietnamiensis (AMMD) T

B. vietnamiensis (TVV75) T

B. vietnamiensis (TVV70)T

B. plantarii (LMG9035) T

$B$. xenovorans (LB400) T

$B$. cenocepacia (KN9) T

B. ferrariae (FeG101) T

B. ambifaria (LMG19182) T

B. oklahomensis (C6786) T

B. ubonensis (GTC-P3-415) T

WC5.4C $L$. ambigua SA

WC5.4d $L$. ambigua SA

WC5.4e $L$. ambigua SA

WC21.1i $L$. pauciflora SA

WC21.1b $L$. pauciflora SA

WC21.1j $L$. pauciflora SA

WC26.1d $L$. meyerianna SA

WC26.1e L. meyerianna SA

WC26.1f $L$. meyerianna SA

$P$. lemoignei (LMG2207)

$R$. detusculanense (APF11)

$R$. solanacearum (R221)

R. pickettii (ATCC27512)

Az. caulinodans (LMG6465)

P. aeruginosa (ATCC10145)

$K$. pneumoniae (AU45) gggccttcta cacaccgcce gtcacaccat gggagttggc tttacccgaa gggccttgta cacaccgccc gtcacaccat gggagtgggt tgctccagaa gggccttgta cacaccgccc gtcacaccat gggagtgggt tgcaaaagaa

1410

gtaggtagct taacc

gtaggtagcc taacc

gtggctagtc taacc

gtggcagtct taacc

gtggctagtc taacc

gtggctagtc taacc

gtggctagtc taacc

gtggctagtc taacc

gtggctagtc taacc

gtggctagtc taacc

gtggctagtc taacc

gtggctagtc taacc

gtggctagtc taacc

gtggctagtc taacc

gtggctagtc taacc

gtggctagtc taacc

gtggctagtc taacc

gtaggtagcc ttaac

gtggctagtc taacc

gtggctagtc taacc

gtggctagtc taacc

gtggetagtc taacc

gtagttagcc taacc

gtggctagtc taacc

gtggctagtc taacc

gtggctagtc taacc

gtggctagtc taacc

gtggctagtc taacc

gtagttagcc ttaac

gtggetagtc taacc

gtggctagtc taacc

gtggctagtc taacc

gtggetagtc taacc

gtggctagtc taacc

gtggctagtc t----

gtggctagtc taacc

gtggctagtc taacc

gtggctagtc taacc

gtggctagtc taacc

gtggctagtc taacc

gtggctagtc taacc

gtggctagtc taacc

gtggctagtc taacc

gtggctagtc taacc

gtggctagtc taacc

gtggctagtc taacc

gtggctagtc taacc

gtggctagtc taacc

gtaggtagcc taacc

gtaggtagcc taacc

gtgggtagtc taacc

gtggctagtc taacc

gtggctagtc taacc

gtggctagtc taacc

gtggctagtc taacc

gtggctagtc taacc

gtaggtagct taacc

gtagttagcc taacc

gtagttagcc taacc

-----tagcc taacc

ggcgttgcgc taacc

gtagctagtc taacc

gtaggtagct taacc 


\section{APPENDIX C: $\alpha$-RHIZOBIA 16S rRNA alignment}

M. mediterraneum (LMG17148) T

$M$. septentrionale (SDW014) T

$M$. temperatum (SDW018) T

$M$. thiogangeticum (SJT) T

$M . t i a n s h a n e n s e$ (A-1BS) T

WC12.1a $L$. sepiaria SA

WC19.1b L. cytisoides SA

WC19.1c $L$. cytisoides SA

WC23.1b L. multiflora SA

WC23.1C $L$. multiflora SA

WC23.1d L. multiflora SA

WC28.1F $L$. sericea SA

WC28.1g $L$. sericea SA

WC28.1h $L$. sericea SA

WC28.1i $L$. sericea SA

WC33b $L$. sericea SA

WC33c $L$. sericea SA

WC33e $L$. sericea SA

WC33h $L$. sericea SA

$S$. americanum (CFNEI156) T

$S$. arboris (LMG14919) T

S. fredii (LMG6217) T

S. kostiense (LMG19227)

S. kummerowiae (CCBAU71714) T

S. meliloti (LMG6133) T

BH1LSC $L$. spinescens SA

BH2LSa $L$. spinescens SA

BH3LSd L. spinescens SA

BW1LSab $L$. spinescens SA

BW2LSa $L$. spinescens SA

BW2LSb $L$. spinescens SA

BW2LSC $L$. spinescens SA

BW3LSd $L$. spinescens SA

B. canariense (MSFC2-M3-R-3) T

$B$. japonicum (LMG6138) T

$B$. liaoningense (LMG18230) T

Betal106d L. pungens SA

Betal106a $L$. pungens SA

NK22 $L$. sericea SA

WC21.1L L. pauciflora SA

XHRla L. simsiana SA

Betal106f $L$. pungens SA

$R$. palustris (ATCC17001)

$M$. nodulans (ORS2060)

$A z$. caulinodans (LMG6465)

$R$. rubrum (LMGATCC11170)

A. magnetotacticum (M58171)

Az. brasilense (NCIMB11860)

Az. Iipoferum (NCIMB11861)

R. acidophila (M34128)

A. clevelandensis (M69186)

S. morelense (LMG21331) T

$M$. amorphae (LMG18977) T

$M$. ciceri (LMG14989) T

M. huakuii (LMG14107) T

M. Loti (LMG6125) T

M. plurifarium (LMG11892) T

B. elkanii (LMG6134) T

B. beta (LMG21987) T

B. yuanmingense (CCBAU10071) T

S. medicae (A321) T

M. mediterraneum (LMG17148) T

$M$. septentrionale (SDW014) T

$M$. temperatum (SDW018) T

$M$. thiogangeticum (SJT) T

$M . t i a n s h a n e n s e(\mathrm{~A}-1 \mathrm{BS}) \mathrm{T}$

WC12.1a L. sepiaria SA

WC19.1b L. cytisoides SA

WC19.1C L. cytisoides SA

WC23.1b $L$. multiflora SA

WC23.1C L. multiflora SA tgcaagtcga gc----gcc- --------- - - c gca--ag gggagcg-gc tgcaagtcga gc----gcc- ---------- -ccgca--ag gggagcg-gc tgcaagtcga gc----gcc- ---------- -ccgca--gg gggagcgtgc tgcaagtcga gc----gcc- ---------- -ccgca--ag gggagcg-gc tgcaagtcga gc----gCC- - - - - - - - - ccgca--ag gggagcg-gc --gcagtcga gc----gcc- ---------- -ccgca--ag gggagcg-gc tgcaagtcga gc----gcc- ---------- -ccgca--ag gggagcg-gc -----gtcga gc----gcc- - ---_--- -ccgca--ag gggagcg-gc tgcaagtcga gc----gCc- --------- -ccgca--ag gggagcg-gc tgcaagtcga gc----gcC- ---------- -ccgca--ag gggagcg-gc tgc-agtcga gc----gcc- ---------- -ccgga--tg gggagcg-gc tgcaagtcga gc----gcc- --------- -ccgca--ag gggagcg-gc tgcaagtcga gc----gcC- ---------- -ccgca--ag gggagcg-gc tgc-agtcga gc----gCC- ---------- -ccgca--ag gggagcg-gc tgcaagtcga gc----gcc- ---------- -ccgca--ag gggagcg-gc

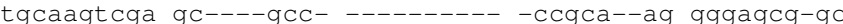
tgcaagtcga gc----gcc- ---------- -ccgca--ag gggagcg-gc --------- gC----gCC- --------- -ccgga--ag gggagcg-gc tgcaagtcga gc----gCC- --------- -ccgca--ag gggagcg-gc tgcaagtcga gc----gCC- --------- -ccgca--ag gggagcg-gc tgcaagtcga gc----gcc- ---------- -ccgca--ag gggagcg-gc tgcaagtcga gc----gcc- --------- -ccgca--ag gggagcg-gc tgcaagtcga ac----gcc- ---------- -ccgca--ag gggagcg-gc tgcaagtcga gc----gcc- -.------- -ccgca--ag gggagcg-gc tgcgagtcga gc----gcc- ---------- -ccgca--ag gggagcg-gc tgcaagtcga gc----gcc- - --------- -ccgca--ag gggagcg-gc tgcaagtcga gc----gcc- ---------- -ccgca--ag gggagcg-gc tgcaagtcga gc----gcc- ---------- -ccgca--ag gggagcg-gc tgcaagtcga gc----gcc- ---------- -ccgca--ag gggagcg-gc tgcaagtcga gc----gcc- - -------- -ccgca--ag gggagcg-gc tgcaagtcga gc----gCc- --------- -ccgca--ag gggagcg-gc tgcaagtcga gc----gcc- ---------- -ccgca--ag gggagcg-gc ----agtcga gcgg--gcg- ---------- -tagcaatac gtcagcg-gc tgcaagtcga gcgg--gcg- ---------- -tagcaatac gtcagcg-gc tgcaagtcga gcgg--gcg- -.------- -tagcaatac gtcagcg-gc tgcaagtcga gcgg--gcg- --------- -tagcaatac gtcagcg-gc -gcgagtcga gcgg--gcg- -.------- -tagcaatac gtcagcg-gc tgcaagtcga gcgg--gcg- - - - - - - - tagcaatac gtcagcg-gc ------tcga gcgg--gca- --------- -tagcaatat gtcagcg-gc ----agtcga gcgg--gcg- - --_-_--- -tagcaatac gtcagcg-gc tgcaagtcga gcgg--gcg- ---------- -tagcaatac gtcagcg-gc tgcaagtcga acgg--gcg- --------- -tagcaatac gtcagtg-gc tgcaagtcga gcgg--gcc- - - - - - - - - ctgcg--gg gtcagcg-gc tgcaagtcga gc----gcc- ---------- -cagca--at gggagcg-gc tgcaagtcga acgc--atc- --------- -cttcg--gg atgagtg-gc tgcaagtcga acga--agt- --------- -cttcg--ga cttagtg-gc tgcaagtcga acga--agg- ---------- -cttcg--gc cttagtg-gc tgcaagtcga acga--ggg- -----tttcc ttcggggggc cttagtg-gc tgcaagtcga acgg--gca- - - - - - - - -tagcaatat gtcagtg-gc tgcaagtcga acgg--gcg- --------- -tagcaatac gtcagtg-gc tgcaagtcga gc----gcc- --------- -ccgca--ag gggagcg-gc tgcaagtcga gc----gCC- ---------- -ccgca--ag gggagcg-gc tgcaagtcga gc----gCc- - -------- -ccgca--ag gggagcg-gC tgcaagtcga gc----g-C- - - - - - - - - ccgca--ag gggagcg-gc tgcaagtcga gc----gcc- --------- -tcgca--a- --gagcg-gc tgcaagtcga gc----gCc- --------- -ccgca--ag gggagcg-gc tgcaagtcga gcgg--gca- -.-_-_-_- -tagcaatat gtcagcg-gc tgcaagtcga gcgg--gcg- ---------- -tagcaatac gtcagcg-gc tgcaagtcga gcgg--gcg- --------- -tagcaatac gtcagcg-gc tgcaagtcga gC----gCC70 80 90

agacgggtga gtaacgcgtg ggaatctacc catctctacg gaacaactcc agacgggtga gtaacgcgtg ggaatctacc catctctacg gaacaactcc agacgggtga gtaacgcgtg ggaatctacc catctctacg gaacaactcc agacgggtga gtaacgcgtg ggaatctacc cagctctacg gaataaccca agacgggtga gtaacgcgtg ggaatctacc catctctacg gagcaactcc agacgggtga gtaacgcttg - gaatctacc catctctacg gaacaactcc agacgggtga gtaaccctt- -aaagctacc catctctacg gaacaactcc agacgggtga gtaa--cggg ggaatctacc catctctacg gaacaactcc agacgggtga gtaacgccgg ggaatctacc catctctacg gaacaactcc agacgggtga gtaacgctt- -ggatctccc catctctacg gaacaactcc 
WC23.1d $L$. multiflora SA WC28.1F $L$. sericea SA WC28.1g $L$. sericea SA WC28.1h L. sericea SA WC28.1i $L$. sericea SA WC33b L. sericea SA WC33C $L$. sericea SA WC33e L. sericea SA WC33h L. sericea SA

$S$. americanum (CFNEI156) T

$S$. arboris (LMG14919) T

S. fredii (LMG6217) T

S. kostiense (LMG19227)

S. kummerowiae (CCBAU71714) T

S. meliloti (LMG6133) T

BH1LSC $L$. spinescens SA

BH2LSa L. spinescens SA

BH3LSd L. spinescens SA

BW1LSab $L$. spinescens SA

BW2LSa $L$. spinescens SA

BW2LSb $L$. spinescens SA

BW2LSC $L$. spinescens SA

BW3LSd $L$, spinescens SA

$B$. canariense (MSFC2-M3-R-3) T

$B$. japonicum (LMG6138) T

$B$. liaoningense (LMG18230) T

Betal106d $L$. pungens SA

Betal106a L. pungens SA

NK22 $L$. sericea SA

WC21.1L L. pauciflora SA

XHRla $L$ simsiana SA

Betal106f $L$. pungens SA

$R$. palustris (ATCC17001)

$M$. nodulans (ORS2060)

Az. caulinodans (LMG6465)

$R$. rubrum (LMGATCC11170)

A. magnetotacticum (M58171)

Az. brasilense (NCIMB11860)

Az. Iipoferum (NCIMB11861)

R. acidophila (M34128)

A. clevelandensis (M69186)

$S$. morelense (LMG21331) T

M. amorphae (LMG18977) T

M. ciceri (LMG14989) T

$M$. huakuii (LMG14107) T

M. loti (LMG6125) T

M. plurifarium (LMG11892) T

B. elkanii (LMG6134) T

B. beta (LMG21987) T

B. yuanmingense (CCBAU10071) T

S. medicae (A321) T

M. mediterraneum (LMG17148) T

$M$. septentrionale (SDW014) T

$M$. temperatum (SDW018) T

$M$. thiogangeticum (SJT) T

$M . t i a n s h a n e n s e$ (A-1BS) T

WC12.1a $L$. sepiaria SA

WC19.1b L. cytisoides SA

WC19.1c $L$. cytisoides SA

WC23.1b $L$. multiflora SA

WC23.1c $L$. multiflora SA

WC23.1d L multiflora SA

WC28.1F $L$. sericea SA

WC28.1g $L$. sericea SA

WC28.1h $L$. sericea SA

WC28.1i $L$. sericea SA

WC33b $L$. sericea SA

WC33c $L$. sericea SA

WC33e $L$. sericea SA

WC33h $L$. sericea SA

S. americanum (CFNEI156) T

S. arboris (LMG14919) T

S. fredii (LMG6217) T

S. kostiense (LMG19227)

S. kummerowiae (CCBAU71714) T agacgggtga gtaacgcgtg tgaatctacc catctctacg gaacaactcc agacgggtga gtaagtttt- -aaaggtacc catctctacg gaacaactcc agacgggtga gtaacgcgtg tgaatctacc catctctacg gaacaactcc agacgggtga gtaa---ggg ggaatctacc catctctacg gaacaactcc agacgggtga gtaacggccg tgaatctacc catctctacg gaacaactcc agacgggtga gtaacgcgtg tgaatctacc catctctacg gaacaactcc agacgggtga gtaacgcgt- --tatctacc catctctacg gaacaactcc agacgggtga gtaa--cggg ggaatctacc catctctacg gaacaactcc agacgggtga gtaaggcctg aaaggctacc catctctacg gaacaactcc agacgggtga gtaacgcgtg ggaatctacc ctttctacg gaataacgca agacgggtga gtaacgcgtg ggaatctacc ctttctacg gaataacgca agacgggtga gtaacgcotg ggaatctacc cttttctacg gaataacgca agacgggtga gtaacgcgtg ggaatctacc cttttctacg gaataacgca agacgggtga gtaacgcgtg ggaatctacc cttttctacg gaataacgca agacgggtga gtaacgcgtg ggaatctacc cttttctacg gaataacgca agacgggtga gtaacgctt- --gatctacc ctttctacg gaataacgca agacgggtga gtaacgcgtg ggaatctacc cttttctacg gaataacgca agacgggtga gtaacgcgtt ggaatctacc cttttctacg gaataacgca agacgggtga gtaacgcgcg ggaatctacc ctttctacg gaataacgca agacgggtga gtaacgcgt- -gaatctacc cttttctacg gaataacgca agacgggtga gtaacgcgtg ggaatctacc ctttctacg gaataacgca agacgggtga gtaacgctt- ggaatctacc ctttctacg gaataacgca agacgggtga gtaacgcct- ggaatctacc cttttctacg gaataacgca agacgggtga gtaacgcgtg ggaacgtacc ttttggttcg gaacaacaca agacgggtga gtaacgcgtg ggaacgtacc ttttggttcg gaacaacaca agacgggtga gtaacgcgtg ggaacgtacc ttttggttcg gaacaacaca agacgggtga gtaa-gcgtg ggaacgtacc ttttggttcg gaacaaccca agacgggtga gc-ctgcatg ggaacgtacc ttttggttcg gaacaaccga agacgggtga gtaat-cgag ggaacgtacc ttttggttcg gaacaacaca agacgggtga gt-acgtgag ggaacgtacc ttttggttcg gaacaactga agacgggtga gcaatgagtg ggaacatacc ttttggttcg gaacaacaca agacgggtga gtaac-catg agaacgtacc ttttggttcg gaacaacaca agacgggtga gtaacgcgtg ggaacgtacc ttttggttcg gaacaacaca agacgggtga gtaacgcgtg ggaacgtgcc cttcggttcg gaacaaccct agacgggtga gtaacgcgtg gggatgtgcc caltggtgcg gaataaccca gcacgggtga gtaacacgtg ggaacgtacc ttggagtgcg gaataatct gcacgggtga gtaacacgtg ggaatatacc tcttggtggg gaataacgtc gcacgggtga gtaacacgtg ggaacctgcc tttcggttcg ggataacgtc gcacgggtga gtaacacgtg ggaacctgcc tttcggttcg gaataacgtc agacgggtga ggaacacgtg ggaacatgcc ctttggttcg gaacaactca agacgggtga gtaacgcgtg ggaacgtacc ttttggttcg gaacaacaca agacgggtga gtaacgcotg ggaatctacc cttttctacg gaataacgca agacgggtga gtaacgcgtg ggaatctacc catctctacg gaacaactcc agacgggtga gtaacgcgtg ggaatctacc catctctacg gaacaactcc agacgggtga gtaacgcgtg ggaatctacc catctctacg gaacaactcc agacgggtga gtaacgcgtg ggaatctacc catctctacg gaacaactcc agacgggtga gtaacgcgtg ggaatctacc catctctacg gaacaactcc agacgggtga gtaacgcgtg ggaacgtacc ttttggttcg gaacaactga agacgggtga gtaacgcgtg ggaacatacc ttttggttcg gaacaacaca agacgggtga gtaacgcgtg ggaacgtacc ttttggttcg gaacaacaca agacgggtga gtaacgcgtg ggaatctacc ctttctacg gaataacgca

120

130

140

gggaaactgg agctaatacc gtatacgtcc ttcggga--- ---------g gggaaactgg agctaatacc gtatacgtcc ttcggga--- ---------g gggaaactgg agctaatacc gtatacgtcc ttcggga--- ---------g gggaaacttg gactaatacc gtatacgtcc tattgga--- ---------g gggaaactgg agctaatacc gtatacgtcc ttcggga--- ---------o gggaaactgg agctaatacc gtatacgtcc ttttgga--- ---------o ggg-aactgg agctaatacc gtatacgtcc ttttgga--- ---------g gggaaactgg agctaatacc gtatacgtcc ttttgga--- ---------o gggaaactgg agctaatacc gtatacgtcc ttttgga--- ---------g gggaaactgg agctaatacc gtatacgtcc ttttgga--- ---------g gggaaactgg agctaatacc gtatacgtcc ttttgga--- ---------g ggg-aactgg aggtaatacc gtatacgtcc ttttgga--- ---------g gggaaactgg agctaatacc gtatacgtcc ttttgga--- ---------g gggaaactgg agctaatacc gtatacgtcc ttttgga--- ---------g gggaaactgg agctaatacc gtatacgtcc ttttgga--- ---------g gggaaactgg agctaatacc gtatacgtcc ttttgga--- ---------o gggaaactgg agctaatacc gtatacgtcc ttttgga--- ---------g gggaaactgg agctaatacc gtatacgtcc ttttgga--- ---------g ggg-aacagg agctaatacc gtatacgtcc ttttgga--- ---------o gggaaacttg tgctaatacc gtatgagccc ttcgggg--- ---------g gggaaacttg tgctaatacc gtataagccc ttcgggg--- ---------g gggaaacttg tgctaatacc gtatgagccc ttcgggg--- $---------g$ gggaaacttg tgctaatacc gtatacgccc ttcgggg--- ---------g gggaaacttg tgctaatacc gtatgagccc ttcgggg--- ---------g 
S. meliloti (LMG6133) T BH1LSC $L$. spinescens SA BH2LSa $L$. spinescens SA BH3LSd $L$. spinescens SA BW1LSab $L$. spinescens SA BW2LSa $L$. spinescens SA BW2LSb $L$. spinescens SA BW2LSC $L$. spinescens SA BW3LSd L. spinescens SA

$B$. canariense (MSFC2-M3-R-3) T

$B$. japonicum (LMG6138) T

B. liaoningense (LMG18230)T

Betal106d $L$. pungens SA

Betal106a $L$. pungens SA

NK22 $L$. sericea SA

WC21.1L L. pauciflora SA

XHRla $L$. simsiana SA

Betal106f $L$. pungens SA

$R$. palustris (ATCC17001)

M. nodulans (ORS2060)

Az. Caulinodans (LMG6465)

$R$. rubrum (LMGATCC11170)

A. magnetotacticum (M58171)

$A z$. brasilense (NCIMB11860)

Az. Iipoferum (NCIMB11861)

R. acidophila (M34128)

A. clevelandensis (M69186)

S. morelense (LMG21331) T

$M$. amorphae (IMG18977) T

M. ciceri (LMG14989) T

$M$. huakuii (LMG14107) T

M. Loti (LMG6125) T

M. plurifarium (LMG11892)T

B. elkanii (LMG6134) T

B. beta (LMG21987) T

$B$. yuanmingense (CCBAU10071) T

S. medicae (A321) T

M. mediterraneum (LMG17148) T

$M$. septentrionale (SDW014) T

$M$. temperatum (SDW018) T

$M$. thiogangeticum (SJT) T

$M . t i a n s h a n e n s e$ (A-1BS) T

WC12.1a $L$. sepiaria SA

WC19.1b $L$. cytisoides SA

WC19.1C L. cytisoides SA

WC23.1b $L$. multiflora SA

WC23.1C L. multiflora SA

WC23.1d L. multiflora SA

WC28.1F $L$. sericea SA

WC28.1g $L$. sericea SA

WC28.1h I sericea SA

WC28.1i $L$. sericea SA

WC33b $L$. sericea SA

WC33C $L$. sericea SA

WC33e $L$. sericea SA

WC33h L. sericea SA

$S$. americanum (CFNEI156) T

S. arboris (LMG14919) T

S. fredii (LMG6217) T

S. kostiense (LMG19227)

S. kummerowiae (CCBAU71714) T

S. meliloti (LMG6133) T

BH1LSc $L$. spinescens SA BH2LSa $L$. spinescens SA BH3LSd $L$. spinescens SA BW1LSab $L$. spinescens SA BW2LSa $L$. spinescens SA BW2LSb $L$. spinescens SA BW2LSC $L$. spinescens SA BW3LSd $L$. spinescens SA

$B$. canariense (MSFC2-M3-R-3) T

B. japonicum (LMG6138) T

B. liaoningense (LMG18230) T

Betal106d L. pungens SA

Betal106a $L$. pungens SA

gggaaacttg tgctaatacc gtatgagccc ttcgggg--- ---------o gggaaacttg tgctaatacc gtatgagccc ttcgggg--- ---------g gggaaacttg tgctaatacc gtatgagccc ttcgggg--- ---------g gggaaacttg tgctaatacc gtatgagccc ttcgggg--- ---------g gggaaacttg tgctaatacc gtatgagccc ttcgggg--- ---------g gggaaacttg tgctaatacc gtatgagccc ttcgggg--- $---------g$ gggaaacttg tgctaatacc gtatgagccc ttcgggg--- ---------g gggaaatttg tgctaatacc gtatgagccc ttcgggg--- ---------g gggaaacttg tgctaatacc gtatgagccc ttcgggg--- ---------o gggaaacttg tgctaatacc ggataagccc ttacggg--- ---------g gggaaacttg tgctaatacc ggataagccc ttacggg--- ---------g gggaaacttg tgctaatacc ggataagccc ttacggg--- ---------og gggaaacttg ggctaatacc ggataagccc ttacggg--- ---------o ggtaaacttg ggctaatacc ggataagccc ttacggg--- ---------g gggaaacttg tgctaatacc ggataagccc ttacggg--- $---------g$ ggcaaacttc agctaatacc ggataagccc ttacggg--- ---------g gggaaacttg tgctaatacc ggataagccc ttacggg--- ---------o gggaaacttg tgctaatacc ggataagccc ttacggg--- $---------g$ gggaaacttg tgctaatacc ggataagccc ttacggg--- ---------g gggaaactag ggctaatacc ggatacgtcc gcaagga--- --------- g gggaaacttg gattaatacc gcatgtgccc ttcgggg--- --------- g tggaaacgag gactaatacc gcatacgccc ttagggg--- ---------g gggaaactga cgctaatacc gcatacgccc ttcgggg--- ---------g tggaaacgga cgctaacacc ggatacgtcc cccagagaga tttgg----tggaaacgga cgctaacacc ggatacgccc tatgggg--- ---------g gggaaacttg agctaatacc ggataagcct ttcgagg--- ---------og gggaaacttg tgctaatacc ggataag-cc ttacggg--- ---------g tggaaacgtg tgctaatacc gtatgagccc ttcgggg--- ---------g gggaaactgg agctaatacc gtatacgtcc ttcggga--- $---------g$ gggaaactgg agctaatacc gtatacgtcc ttcggga--- ---------g gggaaactgg agctaatacc gtatacgtcc ttcggga--- ---------g gggaaactgg agctaatacc gtatacgt-c ttcggga--- ---------g gggaaactgg agctaatacc gtatacgtcc ttcggga--- ---------g gggaaacttc agctaatacc ggataagccc ttacggg--- ---------g gggaaacttg tgctaatacc ggataagccc ttacggg--- ---------g gggaaacttg tgctaatacc ggataagccc ttacggg--- ---------o gggaaacttg tgctaatacc gtataagccc ttcgggg--- ---------g

160 aaa------- -------gat tt--atcgga gatggatgag cccgcgttgg aaa------ -------gat tt--atcgga gatggatgag cccgcgttgg aaa------- -------gat tt--atcgga gttggatgag cccgcgttgg aaa------- -------gat tt--atcgga gatggatgag cccgcgttgg aaa------- -------gat tt--atcgga gatggatgag cccgcgttgg aaa------- -------gat tt--atcgga gatggatgag cccgcgttgg aaa------- ------- gat tt--atcgga gatggatgag cccgcgttgg aaa------ -------gat tt--atcgga gatggatgag cccgcgttgg aaa------- -------gat tt--atcgga gatggatgag cccgcgttgg aaa------- -------gat tt--atcgga gatggatgag cccgcgttgg aaa------ -------gat tt--atcgga gatggatgag cccgcgttgg aaa------- -------gat tt--atcgga gatggatgag cccgcgttgg aaa------- -------gat tt--atcgga gatggatgag cccgcgttgg aaa------- -------gat tt--atcgga gatggatgag cccgcgttgg aaa------- -------gat tt--atcgga gatggatgag cccgcgttgg aaa------ -------gat tt--atcgga gatggatgag cccgcgttgg aaa------- -------gat tt--atcgga gatggatgag cccgcgttgg aaa------- -------gat tt--atcgga gatggatgag cccgcgttgg aaa------ -------gat tt--atcggg aaaggatgag cccgcgttgg aaa------- -------gat tt--atcggg aaaggatgag cccgcgttgg aaa------- -------gat tt--atcggg aaaggatgag cccgcgttgg aaa------- -------gat tt--atcgga gaaggatgag cccgcgttgg aaa------- -------gat tt--atcggg aaaggatgag cccgcgttgg aaa------ -------gat tt--atcggg aaaggatgag cccgcgttgg aaa------- -------gat tt--atcggg aaaggatgag cccgcgttgg aaa------- -------gat tt--atcggg aaaggatgag cccgcgttgg aaa------- -------gat tt--atcggg aaaggatgag cccgcgttgg aaa------- -------gat tt--atcggg aaaggatgag cccgcgttgg aaa------- -------gat tt--atcggg aaaggatgag cccgcgttgg aaa------ -------gat tt--atcggg aaaggatgag cccgcgttgg aaa------- -------gat tt--atcggg aaaggatgag cccgcgttgg aaa------- -------gat tt--atcggg aaaggatgag cccgcgttgg aaa------- -------gat tt--atcgec gaaagatcgg cccgcgtctg aaa------- -------gat tt--atcgcc gaaagatcgg cccgcgtctg aaa------- -------gat tt--atcgcc gaaagatcgg cccgcgtctg aaa------- -------gat tt--atcgcc gaaagatcgg cccgcgtctg aaa------ -------gat tt--atcgec gaaagatcgg cccgcgtctg 
NK22 L. sericea SA WC21.1L L. pauciflora SA XHR1a L. simsiana SA Betal106f L. pungens SA R. palustris (ATCC17001) $M$. nodulans (ORS2060)

$A z$. caulinodans (LMG6465)

R. rubrum (LMGATCC11170)

A. magnetotacticum (M58171)

Az. brasilense (NCIMB11860)

$A z$. lipoferum (NCIMB11861)

R. acidophila (M34128)

A. clevelandensis (M69186)

$S$. morelense (LMG21331) T

M. amorphae (LMG18977) T

M. ciceri (LMG14989) T

$M$. huakuii (LMG14107) T

$M$. loti (LMG6125) T

M. plurifarium (LMG11892) T

B. elkanii (LMG6134) T

B. beta (LMG21987) T

B. yuanmingense (CCBAU10071) T

S. medicae (A321) T

M. mediterraneum (LMG17148) T

$M$. septentrionale (SDW014) T

$M$. temperatum (SDW018) T

$M$. thiogangeticum (SJT) T $M$. tianshanense (A-1BS)T WC12.1a L. sepiaria SA

WC19.1b L. cytisoides SA WC19.1c $L$. cytisoides SA WC23.1b $L$. multiflora SA WC23.1c $L$. multiflora SA WC23.1d $L$. multiflora SA WC28.1F $L$. sericea SA WC28.1g $L$. sericea SA WC28.1h L. sericea SA WC28.1i $L$. sericea SA WC33b L. sericea SA WC33C I sericea SA WC33e L. sericea SA WC33h L. sericea SA $S$. americanum (CFNEI156) T

S. arboris (LMG14919) T

S. fredii (LMG6217) T

S. kostiense (LMG19227)

s. kummerowiae (CCBAU71714) T

S. meliloti (LMG6133) T

BH1LSC $L$. spinescens SA BH2LSa L. spinescens SA BH3LSd $L$ spinescens SA BW1LSab $L$. spinescens SA BW2LSa $L$. spinescens SA BW2LSb $L$. spinescens SA BW2LSC $L$. spinescens SA BW3LSd $L$. spinescens SA B. canariense (MSFC2-M3-R-3) T B. japonicum (LMG6138)T B. liaoningense (LMG18230) T Betal106d L. pungens SA Betall06a L. pungens SA NK22 I sericea SA WC21.1L $L$. pauciflora SA XHR1a L. simsiana SA

Betal106f L. pungens SA $R$. palustris (ATCC17001) $M$. nodulans (ORS2060)

Az. Caulinodans (LMG6465)

$R$. rubrum (LMGATCC11170)

A. magnetotacticum (M58171)

Az. brasilense (NCIMB11860)

$A z$. lipoferum (NCIMB11861)

R. acidophila (M34128)

A. Clevelandensis (M69186)

S. morelense (LMG21331) T aaa------ -------gat tt--atcgec gaaagatcgg cccgcgtctg aaa------- -------gat tt--atcgcc gaaagatcgg cccgcgtctg aaa------ -------gat tt--atcgcc gaaagattgg cccgcgtctg aaa------- -------gat tt--atcgcc gaaagatcgg cccgcgtctg aaa------- -------gat tt--atcgcc gaaagatcgg cccgcgtctg aaa---_-- - ------ggc ttg-actgcc gaaggatcgg cccgcgtctg aaa------ -------gat tt--atcgcc attggatcaa cccgcgtctg aaa------- -------gat tt--atcgct ccaagatcgg cccgcgtccg aaa------ -------gat tt--atcgcc gagagattag cccgcgtccg aga------ -------aag tw--tacgec rwgagagggg cccgcgtccg aaa------ ---------g tt--tacgcc gagagagggg cccgcgtcgg aaa------- -------gat tt--atcgec gaaggagtgg cccgcgtccg aaa------ -------gat tt--atcgec gaaagatcgg cccgcgtctg aaa------- -------gat tt--atcggg aaaggatgag cccgcgttgg aaa------- -------gat tt--atcgga gatggatgag cccgcgttgg aaa------- -------gat tt--atcgga gatggatgag cccgcgttgg aaa------ -------gat tt--atcgga gatggatgag cccgcgttgg aaa------- -------gat tt--atcgga gatggatgag cccgcgttgg aaa------- -------gat tt--atcgga gatggatgag cccgcgttgg aaa------ -------gat tt--atcgec gaaagatcgg cccgcgtctg aaa------- -------gat tt--atcgec gaaagattgg cccgcgtctg aaa------- -------gat tt--atcgcc gaaagatcgg cccgcgtctg aaa------ -------gat tt--atcggg aaaggatgag cccgcgttgg

210 220 230 240 250 attagctagt tggtggggta atggcctacc aaggcgacga tccatagctg attagctagt tggtggggta atggcctacc aaggcgacga tccatagctg attagctagt tggtggggta atggcctacc aaggcgacga tccatagctg attagctagt tggtgaggta atggctcacc aaggcgacga tccatagctg attagctagt tggtggggta atggcctacc aaggcgacga tccatagctg attagctagt tggtggggta atggcctacc aaggcgacga tccatagctg attcgctagt tggtggggta atggcctacc aaggcgacga tccatagctg attagctagt tggtggggta atggcctacc aaggcgacga tccatagctg attagctagt tggtggggta atggcctacc aaggcgacga tccatagctg attagctagt tggtggggta atggcctacc aaggcgacga tccatagctg attagctagt tggtggggta atggcctacc aaggcgacga tccatagctg attttctagt tggtggggta atggcctacc aaggcgacga tccatagctg attagctagt tggtggggta atggcctacc aaggcgacga tccatagctg attagctagt tggtggggta atggcctacc aaggcgacga tccatagctg attagcttgt tggtggggta atggcctacc aaggcgacga tccatagctg attagctagt tggtggggta atggcctacc aaggcgacga tccatagctg attagctagt tggtggggta atggcctacc aaggcgacga tccatagctg attagctagt tggtggggta atggcctacc aaggcgacga tccatagctg attagctagt tggtggggta atggcctacc aaggcgacga tccatagctg attagctagt tggtggggta aaggcctacc aaggcgacga tccatagctg attagctagt tggtggggta aaggcctacc aaggcgacga tccatagctg attagctagt tggtggggta aaggcctacc aaggcgacga tccatagctg attagctagt tggtggggta aaggcctacc aaggcgacga tccatagctg attagctagt tggtggggta aaggcctacc aaggcgacca tccatagctg attagctagt tggtggggta aaggcctacc aaggcgacga tccatagctg attagctagt tggtggggta aaggcctacc aaggcgacga tccatagctg attagctagt tggtggggta aaggcctacc aaggcgacga tccatagctg attagctagt tggtggggta aaggcctacc aaggcgacga tccatagctg attagctagt tggtggggta aaggcctacc aaggcgacga tccatagctg atta-ctagt tggtggggta aaggcctacc aaggcgacga tccatagctg attagctagt tggtggggta aaggcctacc aaggcgacga tccatagctg attagctagt tggtggggta aaggcctacc aaggcgacga tccatagctg attagctagt tggtggggta aaggcctacc aaggcgacga tccatagctg attagctagt tggtgaggta atggctcacc aaggcgacga tcagtagctg attagctagt tggtgaggta atggctcacc aaggcgacga tcagtagctg attagctagt tggtgaggta atggctcacc aaggcgacga tcagtagctg attagctagt tggtgaggta atggctcacc aaggcgacga tcagtagctg attagctagt tggtgaggta atggctcacc aaggcgacga tcagtagctg attagctagt tggtagggta atggcctacc aaggcgacga tcagtagctg attagctagt tggtgaggta atggctcacc aaggcgacga tcagtagctg attagctagt tggtagggta atggcctacc aaggcgacga tcagtagctg attagctagt tggtgaggta atggctcacc aaggcgacga tcagtagctg attagctagt tggtgaggta atggctcacc aaggcgacga tcagtagctg attagctggt tggtggggta acggctcacc aaggcgacga tcagtagctg attagctagt tggtgaggta aaggctcacc aaggcgacga tcagtagctg attagctagt tggcggggta atggcccacc aaggcgacga tcggtagctg attagctagt tggtgaggta atggctcacc aaggcgacga tcggtagctg attaggtagt tggtggggta atggcccacc aagccgacga tcggtagctg attaggtagt tggtgaggta atggctcacc aagcctgcga tccgtagctg attagctagt tggtgaggta atggctcacc aaggcgacga tcgg-agctattagctagt tggtgaggta acggctcacc aaggcgacga tcagtagctg attagctagt tggtggggta aaggcctacc aaggcgacga tccatagctg 
M. amorphae (LMG18977)T

M. ciceri (LMG14989)T

M. huakuii (LMG14107) T

M. loti (LMG6125) T

M. plurifarium (LMG11892) T

B. elkanii (LMG6134) T

B. beta (LMG21987) T

B. yuanmingense (CCBAU10071) T

S. medicae (A321) T

M. mediterraneum (LMG17148) T

M. septentrionale (SDW014) T

$M$. temperatum (SDW018) T

$M$. thiogangeticum (SJT) T

M.tianshanense (A-1BS) T

WC12.1a L. sepiaria SA

WC19.1b L. cytisoides SA

wC19.1c $L$. cytisoides SA

WC23.1b L. multiflora SA

WC23.1c $L$. multiflora SA

WC23.1d $L$. multiflora SA

WC28.1F L. sericea SA

WC28.1g $L$. sericea SA

WC28.1h L. sericea SA

WC28.1i L. sericea SA

WC33b $L$. sericea SA

WC33c $L$. sericea SA

WC33e $L$. sericea SA

WC33h L. sericea SA

$S$. americanum (CFNEI156) T

$S$. arboris (LMG14919) T

$S$. fredii (LMG6217) T

s. kostiense (LMG19227)

S. kummerowiae (CCBAU71714) T

S. meliloti (LMG6133) T

BH1LSC L. spinescens SA BH2LSa L. spinescens SA BH3LSd $L$. spinescens SA BW1LSab $L$. spinescens SA BW2LSa L. spinescens SA BW2LSb $L$. spinescens SA BW2LSC $L$. spinescens SA BW3LSd $L$. spinescens SA B. canariense (MSFC2-M3-R-3) T

B. japonicum (LMG6138) T B. liaoningense (LMG18230) T Betall06d L. pungens SA Betal106a L. pungens SA NK22 L. sericea SA

WC21.1L $L$. pauciflora SA

XHR1a L. simsiana SA

Betal106f L. pungens SA

$R$. palustris (ATCC17001)

$M$. nodulans (ORS2060)

Az. Caulinodans (LMG6465)

$R$. rubrum (LMGATCC11170)

A. magnetotacticum (M58171)

Az. brasilense (NCIMB11860)

$A z$. lipoferum (NCIMB11861)

R. acidophila (M34128)

A. Clevelandensis (M69186)

S. morelense (LMG21331) T

$M$. amorphae (LMG18977) T

M. Ciceri (LMG14989) T

M. huakuii (LMG14107) T

M. loti (LMG6125) T

M. plurifarium (LMG11892) T

B. elkanii (LMG6134) T

B. beta (LMG21987) T

B. yuanmingense (CCBAU10071) T

S. medicae (A321) T

M. mediterraneum (LMG17148) T

$M$. septentrionale (SDW014) T

M. temperatum (SDW018) T attagctagt tggtggggta atggcctacc aaggcgacga tccatagctg attagctagt tggtggggta atggcctacc aaggcgacga tccatagctg attagctagt tggtggggta atggcctacc aaggcgacga tccatagctg attagctagt tggtggggta atggcctacc aaggcgacga tccatagctg attagctagt tggtggggta atggcctacc aaggcgacga tccatagctg attagctagt tggtgaggta atggctcacc aaggcgacga tcagtagctg attagctagt tggtagggta atggcctacc aaggcgacga tcagtagctg attagctagt tggtgaggta atggctcacc aaggcgacga tcagtagctg attagctagt tggtggggta aaggcctacc aaggcgacga tccatagctg

$$
260
$$

280

290

300

gtctgagagg atgatcagcc acattgggac tgagacacgg cccaaactcc gtctgagagg atgatcagcc acactgggac tgagacacgg cccagactcc gtctgagagg atgatcagcc acattgggac tgagacacgg cccaaactcc gtctgagagg atgatcagcc acactgggac tgagacacgg tccagactcc gtctgagagg atgatcagcc acattgggac tgagacacgg cccaaactcc gtctgagagg atgatcagcc acactgggac tgagacacgg cccagactcc gtctgagagg atgatcagcc acactgggac tgagacacgg cccagactcc gtctgagagg atgatcagcc acactgggac tgagacacgg cccagactcc gtctgagagg atgatcagcc acactgggac tgagacacgg cccagactcc gtctgagagg atgatcagcc acactgggac tgagacacgg cccagactcc gtctgagagg atgatcagcc acactgggac tgagacacgg cccagactcc gtctgagagg atgatcagcc acactgggac tgagacacgg cccagactcc gtctgagagg atgatcagcc acactgggac tgagacacgg cccagactcc gtctgagagg atgatcagc acactgggac tgagacacgg cccagactcc gtctgagagg atgatcagcc acactgggac tgagacacgg cccagactcc gtctgagagg atgatcagcc acactgggac tgagacacgg cccagactcc gtctgagagg atgatcagcc acactgggac tgagacacgg cccagactcc gtctgagagg atgatcagcc acactgggac tgagacacgg cccagactcc gtctgagagg atgatcagcc acactgggac tgagacacgg cccagactcc gtctgagagg atgatcagcc acattgggac tgagacacgg cccaaactcc gtctgagagg atgatcagcc acattgggac tgagacacgg cccaaactcc gtctgagagg atgatcagcc acattgggac tgagacacgg cccaaactcc gtctgagagg atgatcagc acattgggac tgagacacgg cccaaactcc gtctgagagg atgatcagcc acattgggac tgagacacgg cccaaactcc gtctgagagg atgatcagcc acattgggac tgagacacgg cccaaactcc gtctgagagg atgatcagcc acattgggac tgagacacgg cccaaactcc gtctgagagg atgatcagcc acattgggac tgagacacgg cccaaactcc gtctgagagg atgatcagcc acattgggac tgagacacgg cccaaactcc gtctgagagg atgatcagcc acattgggac tgagacacgg cccaaactcc gtctgagagg atgatcagcc acattgggac tgagacacgg cccaaactcc gtctgagagg atgatcagcc acattgggac tgagacacgg cccaaactcc gtctgagagg atgatcagcc acattgggac tgagacacgg cccaaactcc gtctgagagg atgatcagcc acattgggac tgagacacgg cccaaactcc gtctgagagg atgatcagcc acattgggac tgagacacgg cccaaactcc gtctgagagg atgatcagcc acattgggac tgagacacgg cccaaactcc gtctgagagg atgatcagcc acattgggac tgagacacgg cccaaactcc gtctgagagg atgatcagcc acattgggac tgagacacgg cccaaactcc gtctgagagg atgatcagcc acattgggac tgagacacgg cccaaactcc gtctgagagg atgatcagcc acattgggac tgagacacgg cccaaactcc gtctgagagg atgatcagcc acattgggac tgagacacgg cccaaactcc gtctgagagg atgatcagcc acattgggac tgagacacgg cccaaactcc gtctgagagg atgatcagcc acattgggac tgagacacgg cccaaactcc gtctgagagg atgatcagcc acattgggac tgagacacgg cccaaactcc gtctgagagg atgatcagcc acactgggac tgagacacgg cccagactcc gtctgagagg atgatcagcc acactgggac tgagacacgg cccagactcc gtctgagagg atggccagcc acactgggac tgagacacgg cccagactcc gtctgagagg atgatcagcc acactgggac tgagacacgg cccagactcc gtctgagagg atgatcagcc acactgggac tgagacacgg cccagactcc gtctgagagg atgatcagcc acactgggac tgagacacgg cccagactcc gtctgagagg atgatcagcc acattgggac tgagacacgg cccaaactcc gtctgagagg atgatcagcc acattgggac tgagacacgg cc--aactcc gtctgagagg atgatcagcc acattgggac tgagacacgg cccaaactcc gtctgagagg atgatcagcc acactgggac tgagacacgg cccagactcc gtctgagagg atgatcagcc acattgggac tgagacacgg cccaaactcc gtctgagagg atgatcagcc acactgggac tgagacacgg cccagactcc gtctgagagg atgatcagc acattgggac tgagacacgg cccaaactcc gtctgagagg atgatcagcc acactgggac tgagacacgg cccagactcc gtctgagagg atgatcagcc acattgggac tgagacacgg cccaaactcc gtctgagagg atgatcagcc acattgggac tgagacacgg cccaaactcc gtctgagagg atgatcagcc acattgggac tgagacacgg cccaaactcc gtctgagagg atgatcagcc acattgggac tgagacacgg cccaaactcc

$$
310
$$

350

tacgggaggc agcagtgggg aatattggac aatgggcgca agcctgatcc tacgggaggc agcagtgggg aatattggac aatgggcgaa agcctgatcc tacgggaggc agcagtgggg aatattggac aatgggcgca agcctgatcc 
M. thiogangeticum (SJT)T $M . t i a n s h a n e n s e$ (A-1BS) T WC12.1a $L$. sepiaria SA WC19.1b $L$. cytisoides SA WC19.1C $L$. cytisoides SA WC23.1b L. multiflora SA WC23.1c L. multiflora SA WC23.1d L. multiflora SA WC28.1F $L$. sericea SA WC28.1g $L$. sericea SA WC28.1h $L$. sericea SA WC28.1i $L$, sericea SA WC33b $L$. sericea SA WC33C L. sericea SA WC33e $L$. sericea SA WC33h $L$. sericea SA $S$. americanum (CFNEI156) T

$S$. arboris (LMG14919) T

$S$. fredii (LMG6217) T

S. kostiense (LMG19227)

S. kummerowiae (CCBAU71714) T S. meliloti (LMG6133) T BH1LSC $L$. spinescens SA BH2LSa $L$. spinescens SA BH3LSd $L$. spinescens SA BW1LSab $L$. spinescens SA BW2LSa $L$. spinescens SA BW2LSb $L$. spinescens SA BW2LSC $L$. spinescens SA BW3LSd $L$. spinescens SA $B$. canariense (MSFC2-M3-R-3) T B. japonicum (LMG6138) T B. liaoningense (LMG18230) T Betal106d L. pungens SA Betal106a L. pungens SA NK22 $L$. sericea SA WC21.1L L. pauciflora SA XHRla L. simsiana SA Betal106f $L$. pungens SA $R$. palustris (ATCC17001) $M$. nodulans (ORS2060) Az. caulinodans (LMG6465)

$R$. rubrum (LMGATCC11170)

A. magnetotacticum (M58171)

Az. brasilense (NCIMB11860)

$A z$. Iipoferum (NCIMB11861)

$R$. acidophila (M34128)

A. Clevelandensis (M69186)

S. morelense (LMG21331) T

$M$. amorphae (LMG18977) T

M. ciceri (LMG14989) T

M. huakuii (LMG14107) T

M. loti (LMG6125) T

M. plurifarium (LMG11892) T

B. elkanii (LMG6134) T

B. beta (LMG21987) T

$B$. yuanmingense (CCBAU10071) T

S. medicae (A321) T

M. mediterraneum (LMG17148) T

$M$. septentrionale (SDW014)T

$M$. temperatum (SDW018) T

$M$. thiogangeticum (SJT) T $M$. tianshanense (A-1BS) T WC12.1a $L$. sepiaria SA

WC19.1b $L$. cytisoides SA WC19.1C $L$. cytisoides SA WC23.1b L. multiflora SA

WC23.1c L. multiflora SA

WC23.1d L. multiflora SA

WC28.1F $L$. sericea SA

WC28.1g $L$. sericea SA WC28.1h $L$. sericea SA WC28.1i $L$ sericea SA WC33b L. sericea SA WC33C L. sericea SA tacgggaggc agcagtgggg aatattggac aatgggcgca agcctgatcc tacgggaggc agcagtgggg aatattggac aatgggcgaa agcctgatcc tacgggaggc agcagtgggg aatattggac aatgggcgca agcctgatcc tacgggaggc agcagtgggg aatattggac aatgggcgca agcctgatcc tacgggaggc agcagtgggg aatattggac aatgggcgca agcctgatcc tacgggaggc agcagtgggg aatattggac aatgggcgca agcctgatcc tacgggaggc agcagtgggg aatattggac aatgggcgca agcctgatcc tacgggaggc agcagtgggg aatattggac aatgggcgca agcctgatcc tacgggaggc agcagtgggg aatattggac aatgggcgca agcctgatcc tacgggaggc agcagtgggg aatattggac aatgggcgca agcctgatcc tacgggaggc agcagtgggg atattggac aatgggcgca agcctgatcc tacgggaggc agcagtgggg aatattggac aatgggcgca agcctgatcc tacgggaggc agcagtgggg aatattggac aatgggcgca agcctgatcc tacgggaggc agcagtgggg aatattggac aatgggcgca agcctgatcc tacgggaggc agcagtgggg aatattggac aatgggcgca agcctgatcc tacgggaggc agcagtgggg aatattggac aatgggcgca agcctgatcc tacgggaggc agcagtgggg aatattggac aatgggcgca agcctgatcc tacgggaggc agcagtgggg aatattggac aatgggcgca agcctgatcc tacgggaggc agcagtgggg aatattggac aatgggcgca agcctgatcc tacgggaggc agcagtgggg aatattggac aatgggcgca agcctgatcc tacgggaggc agcagtgggg aatattggac aatgggcgca agcctgatcc tacgggaggc agcagtgggg aatattggac aatgggcgca agcctgatcc tacgggaggc agcagtgggg aatattggac aatgggcgca agcctgatcc tacgggaggc agcagtgggg aatattggac aatgggcgca agcctgatcc tacgggaggc agcagtgggg aatattggac aatgggcgca agcctgatcc tacgggaggc agcagtgggg aatattggac aatgggcgca agcctgatcc tacgggaggc agcagtgggg aatattggac aatgggcgca agcctgatcc tacgggaggc agcagtgggg aatattggac aatgggcgca agcctgatcc tacgggaggc agcagtgggg aatattggac aatgggcgca agcctgatcc tacgggaggc agcagtgggg aatattggac aatgggcgca agcctgatcc tacgggaggc agcagtgggg aatattggac aatgggggca accctgatcc tacgggaggc agcagtgggg aatattggac aatgggggca accctgatcc tacgggaggc agcagtgggg aatattggac aatgggggca accctgatcc tacgggaggc agcagtgggg aatattggac aatgggcgca agcctgatcc tacgggaggc agcagtgggg aatattggac aatgggcgca agcctgatcc tacgggaggc agcagtgggg aatattggac aatgggggca accctgatcc tacgggaggc agcagtgggg aatattggac aatgggcgca agcctgatcc tacgggaggc agcagtgggg atattggac atgggggca accctgatcc tacgggaggc agcagtgggg aatattggac aatgggcgca agcctgatcc tacgggaggc agcagtgggg aatattggac aatgggggaa accctgatcc tacgggaggc agcagtgggg aatattggac aatgggcgca agcctgatcc tacgggaggc agcagtgggg aatattggac aatgggcgca agcctgatcc tacgggaggc agcagtgggg aatattgcgc aatgggggca accctgacgc tacgggaggc agcagtgggg aatattggac aatgggcgaa agcctgatcc tacgggaggc agcagtgggg aatattggac aatgggggca accctgatcc tacgggaggc agcagtgggg aatattggac aatgggcgca agcctgatcc tacgggaggc agcagtgggg aatattggac aatgggcgca agcc-gatcc tacgggaggc agcagtgggg aatattggac aatgggggca accct-atcc tacgggaggc agcagtgggg atattggac aatgggcgca agcctgatcc tacgggaggc agcagtgggg aatattggac aatgggcgaa agcctgatcc tacgggaggc agcagtgggg aatattggac aatgggcgaa agcctgatcc tacgggaggc agcagtgggg aatattggac aatgggcgca agcctgatcc tacgggaggc agcagtgggg aatattggac aatgggcgaa agcctgatcc tacgggaggc agcagtgggg aatattggac aatgggcgaa agcctgatcc tacgggaggc agcagtgggg aatattggac aatgggcgca agcctgatcc tacgggaggc agcagtgggg aatattggac aatgggggca accctgatcc tacgggaggc agcagtgggg aatattggac aatgggggca accctgatcc tacgggaggc agcagtgggg aatattggac aatgggcgca agcctgatcc

360 370 380 390 400 agccatgccg cgtgagtgat gaaggcccta gggttgtaaa gctcttca agccatgccg cgtgagtgat gaagg-ccta gggttgtaaa gctctttcaa agccatgccg cgtgagtgat gaaggcccta gggttgtaaa gctctttcaa agccatgccg cgtgagtgat gaaggcccta gggttgtaaa gctctttcac agccatgccg cgtgagtgat gaaggcccta gggttgtaaa gctcttcaa agccatgccg cgtgagtgat gaaggcccta gggttgtaaa gctctttcaa agccatgccg cgtgagtgat gaaggcccta gggttgtaaa gctctttcaa agccatgccg cgtgagtgat gaaggcccta gggttgtaaa gctctttcaa agccatgccg cgtgagtgat gaaggcccta gggttgtaaa gctctttcaa agccatgccg cgtgagtgat gaaggcccta gggttgtaaa gctctttcaa agccatgccg cgtgagtgat gaaggcccta gggttgtaaa gctctttcaa agccatgccg cgtgagtgat gaaggcccta gggttgtaaa gctctttcaa agccatgccg cgtgagtgat gaaggcccta gggttgtaaa gctctttcaa agccatgccg cgtgagtgat gaaggcccta gggttgtaaa gctctttcaa agccatgccg cgtgagtgat gaaggcccta gggttgtaaa gctctttcaa agccatgccg cgtgagtgat gaaggcccta gggttgtaaa gctctttcaa agccatgccg cgtgagtgat gaaggcccta gggttgtaaa gctctttcaa 
WC33e I, sericea SA

WC33h $L$. sericea SA

S. americanum (CFNEI156) T

$S$. arboris (LMG14919) T

S. fredii (LMG6217) T

S. kostiense (LMG19227)

S. kummerowiae (CCBAU71714) T

S. meliloti (LMG6133) T

BH1LSC $L$. spinescens SA

BH2LSa $L$. spinescens SA

BH3LSd $L$. spinescens SA

BW1LSab $L$. spinescens SA

BW2LSa $L$. spinescens SA

BW2LSb $L$. spinescens SA

BW2LSC $L$. spinescens SA

BW3LSd $L$. spinescens SA

$B$. canariense (MSFC2-M3-R-3) T

B. japonicum (LMG6138) T

B. liaoningense (LMG18230) T

Betallo6d L. pungens SA

Betal106a L. pungens SA

NK22 $L$. sericea SA

WC21.1L $L$. pauciflora SA

XHR1a L. simsiana SA

Betal106f L. pungens SA

$R$. palustris (ATCC17001)

$M$. nodulans (ORS2060)

Az. caulinodans (LMG6465)

R. rubrum (LMGATCC11170)

A. magnetotacticum (M58171)

$A z$. brasilense (NCIMB11860)

Az. lipoferum (NCIMB11861)

R. acidophila (M34128)

A. Clevelandensis (M69186)

S. morelense (LMG21331) T

M. amorphae (LMG18977) T

M. ciceri (LMG14989) T

M. huakuii (LMG14107) T

M. Loti (LMG6125) T

M. plurifarium (LMG11892) T

B. elkanii (LMG6134) T

B. beta (LMG21987) T

B. yuanmingense (CCBAU10071) T

S. medicae (A321) T

M. mediterraneum (LMG17148) T

$M$. septentrionale (SDW014) T

$M$. temperatum (SDW018) T

$M$. thiogangeticum (SJT) T

$M . t i a n s h a n e n s e(\mathrm{~A}-1 \mathrm{BS}) \mathrm{T}$

WC12.1a $L$. sepiaria SA

WC19.1b L. cytisoides SA

WC19.1c $L$. cytisoides SA

WC23.1b L. multiflora SA

WC23.1C $L$. multiflora SA

WC23.1d L. multiflora SA

WC28.1F $L$. sericea SA

WC28.1g $L$. sericea SA

WC28.1h $L$. sericea SA

WC28.1i L. sericea SA

WC33b L. sericea SA

WC33C L. sericea SA

WC33e I. sericea SA

WC33h L. sericea SA

S. americanum (CFNEI156) T

$S$. arboris (LMG14919) T

S. fredii (LMG6217) T

S. kostiense (LMG19227)

S. kummerowiae (CCBAU71714) T

S. meliloti (LMG6133) T

BH1LSC L. spinescens SA

BH2LSa $L$. spinescens SA

BH3LSd $L$. spinescens SA

BW1LSab $L$. spinescens SA

BW2LSa $L$. spinescens SA

BW2LSb L. spinescens SA

agccatgccg cgtgagtgat gaaggcccta gggttgtaaa gctctttcaa agccatgccg cgtgagtgat gaaggcccta gggttgtaaa gctctttcaa agccatgccg cgtgagtgat gaaggcccta gggttgtaaa gctctttcac agccatgccg cgtgagtgat gaaggcccta gggttgtaaa gctctttcac agccatgccg cgtgagtgat gaaggcccta gggttgtaaa gctctttcac agccatgccg cgtgagtgat gaaggcccta gggttgtaaa gctctttcac agccatgccg cgtgagtgat gaaggcccta gggttgtaaa gctctttcac agccatgccg cgtgagtgat gaaggcccta gggttgtaaa gctctttcac agccatgccg cgtgagtgaa gaaggcccta gggttgtaaa gctctttcac agccatgccg cgtgagtgaa gaaggcccta gggttgtaaa gctctttcac agccatgccg cgtgagtgaa gaaggcccta gggttgtaaa gctctttcac agccatgccg cgtgagtgaa gaaggcccta gggttgtaaa gctctttcac agccatgccg cgtgagtgaa gaaggcccta gggttgtaaa gctctttcac agccatgccg cgtgagtgaa gaaggcccta gggttgtaaa gctctttcac agccatgccg cgtgagtgat gaaggcccta gggttgtaaa gctctttcac agccatgccg cgtgagtgaa gaaggcccta gggttgtaaa gctctttcac agccatgccg cgtgagtgat gaaggcccta gggttgtaaa gctcttttgt agccatgccg cgtgagtgat gaaggcccta gggttgtaaa gctcttttgt agccatgccg cgtgagtgat gaaggcccta gggttgtaaa gctcttttgt agccatgccg cgtgagtgat gaaggcccta gggttgtaaa gctcttttgt agccatgccg cgtgagtgat gaaggcccta gggttgtaaa gctcttttgt agccatgccg cgtgagtgat gaaggcccta gggttgtaaa gctcttttgt agccatgccg cgtgagtgat gaaggcccta gggttgtaaa gctcttttgt agccatgccg cgtgagtgat gaaggcccta gggttgtaaa gctcttttgt agccatgccg cgtgagtgat gaaggcccta gggttgtaaa gctcttttgt agccatgccg cgtgagtgat gaaggcccta gggttgtaaa gct-ttttgt agccatgccg cgtgagtgat gacggcctta gggttgtaaa gctctttct agccatgccg cgtgagtgat gaaggcctta gggttgtaaa gctctttcgc agccatgccg cgtgagtgaa gaaggccttc gggttgtaaa gctctttcgg agccatgccg cgtgagtgat gaaggcctta gggttgtaaa gctctttcac agcaatgccg cgtgagtgat gaaggcctta gggttgtaaa gctctttcgc agcaatgccg cgtgagtgat gaaggcctta gggttgtaaa gctctttcgc agccatgccg cgtgagtgat gacggcccta gggttgtaaa gctctttcac agccatgccg cgtgagtgat gaaggcccta gggttgtaaa gctcttttgt agccatgccg cgtgagtgat gaaggcccta gggttgtaaa gctctttcac agccatgccg cgtgagtgat gaaggcccta gggttgtaaa gctctttcaa agccatgccg cgtgagtgat gaaggtccta gggttgtaaa gctctttcaa agccatgccg cgtgagtgat gaaggcccta gggttgtaaa gctctttcaa agccatgccg cgtgagtgat gaaggcccta gggttgtaaa gctctttcaa agccatgccg cgtgagtgat gaaggcccta gggttgtaaa gctctttcaa agccatgccg cgtgagtgat gaaggcccta gggttgtaaa gctcttttgt agccatgccg cgtgagtgat gaaggcccta gggttgtaaa gctcttttgt agccatgccg cgtgagtgat gaaggcccta gggttgtaaa gctcttttgt agccatgccg cgtgagtgat gaaggcccta gggttgtaaa gctctttcac

\begin{tabular}{|c|c|c|c|c|}
\hline 410 & 420 & 430 & 440 & 45 \\
\hline & $\mathrm{a} a--$ & -- & & 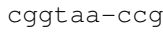 \\
\hline at & $\mathrm{a} a--$ & ---- & $-------\operatorname{tg} a$ & cggtaa-c \\
\hline aa & aa--- & ---- & $-------\operatorname{tg} a$ & taa-c \\
\hline & $a a---$ & ---- & $-------\operatorname{tg} a$ & - \\
\hline t & $a a---$ & ------ & $-------\operatorname{tg} a$ & cggtaa-c \\
\hline at & $a a---$ & ----- & $-------\operatorname{tg} a$ & ggtaa-c \\
\hline & $a a---$ & ----- & $-------\operatorname{tg} a$ & $1-$ \\
\hline t & aa--- & ------ & $-------\operatorname{tg} a$ & $=a-c$ \\
\hline t & $\mathrm{a} a---$ & ------ & $-------\operatorname{tg} a$ & $a-c$ \\
\hline 1 & $a a---$ & & ------- & $1-c$ \\
\hline$a t$ & $\mathrm{a} a--$ & -- & $-------\operatorname{tg} a$ & $d-$ \\
\hline & $\mathrm{a} a---$ & ----- & $-------\operatorname{tg} a$ & $a-c$ \\
\hline & $\mathrm{a} a----$ & ------ & $-------\operatorname{tg} a$ & $a-c$ \\
\hline & $a a--$ & -- & $-------\operatorname{tg} a$ & $-c$ \\
\hline at & aa--- & ----- & $-------\operatorname{tg} a$ & $a-c$ \\
\hline t & aa---- & ------ & $-------\operatorname{tg} a$ & $a-c$ \\
\hline t & $a a---$ & --- & ------- & $a-c$ \\
\hline da & $a a_{---}$ & ----- & $-------\operatorname{tg} a$ & $a-c$ \\
\hline & $\mathrm{a} a----$ & ------ & $-------\operatorname{tg} a$ & $a a-c$ \\
\hline a & $\mathrm{a} a----$ & ------ & $-------\operatorname{tg} a$ & $a a-c$ \\
\hline a & $a a---$ & & $-------\operatorname{tg} a$ & $a-c$ \\
\hline la & $\mathrm{a} a----$ & ------ & $-------\operatorname{tg} a$ & $a a-c$ \\
\hline a & aa--- & ------ & $-------\operatorname{tg} a$ & $a a-c$ \\
\hline aac & $a a---$ & -- & $-------t$ & $a a-c$ \\
\hline aac & $\mathrm{a} a-----$ & ------ & $-------t$ & $a a-c$ \\
\hline a a & a $a----$ & ------ & $-------t$ & $a a-c$ \\
\hline raa & a $a----$ & ----- & $-------\operatorname{tg} a$ & cggtaa-c \\
\hline aac & $a a----$ & ------ & $-------t$ & taa-c \\
\hline & aa--- & ------- & $-------t$ & $a a-$ \\
\hline 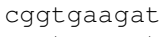 & $\mathrm{a} a----$ & ------ & $-------\operatorname{tg} a$ & aa- \\
\hline & & & & \\
\hline
\end{tabular}


BW2LSC $L$. spinescens SA BW3LSd $L$. spinescens SA $B$. canariense (MSFC2-M3-R-3) T B. japonicum (LMG6138) T B. liaoningense (LMG18230) T Betal106d L. pungens SA Betal106a $L$. pungens SA NK22 $L$. sericea SA WC21.1L $L$. pauciflora SA XHRla L. simsiana SA Betal106f $L$. pungens SA $R$. palustris (ATCC17001) $M$. nodulans (ORS2060) Az. Caulinodans (LMG6465)

R. rubrum (LMGATCC11170)

A. magnetotacticum (M58171)

Az. brasilense (NCIMB11860)

Az. Iipoferum (NCIMB11861)

R. acidophila (M34128)

A. clevelandensis (M69186)

$S$. morelense (LMG21331) T

$M$. amorphae (LMG18977) T

M. ciceri (LMG14989) T

M. huakuii (LMG14107) T

M. Loti (LMG6125) T

M. plurifarium (LMG11892) T

B. elkanii (LMG6134) T

B. beta (LMG21987) T

B. yuanmingense (CCBAU10071) T

S. medicae (A321) T

M. mediterraneum (LMG17148) T

$M$. septentrionale (SDW014) T

$M$. temperatum (SDW018) T

$M$. thiogangeticum (SJT) T

M.tianshanense (A-1BS) T

WC12.1a L. sepiaria SA

WC19.1b $L$. cytisoides SA

WC19.1c $L$. cytisoides SA

WC23.1b L. multiflora SA

WC23.1c I multiflora SA

WC23.1d L. multiflora SA

WC28.1F $L$. sericea SA

WC28.1g $L$. sericea SA

WC28.1h $L$. sericea SA

WC28.1i L. sericea SA

WC33b $L$. sericea SA

WC33c L. sericea SA

WC33e $L$. sericea SA

WC33h L. sericea SA

S. americanum (CFNEI156) T

$S$. arboris (LMG14919) T

S. fredii (LMG6217) T

S. kostiense (LMG19227)

S. kummerowiae (CCBAU71714) T

S. meliloti (LMG6133) T

BH1LSC $L$. spinescens SA

BH2LSa $L$. spinescens SA BH3LSd $L$. spinescens SA BW1LSab $L$. spinescens SA

BW2LSa $L$. spinescens SA BW2LSb $L$. spinescens SA BW2LSC $L$. spinescens SA BW3LSd $L$. spinescens SA $B$. canariense (MSFC2-M3-R-3) T B. japonicum (LMG6138) T B. liaoningense (LMG18230) T Betal106d L. pungens SA Betal106a L. pungens SA NK22 $L$. sericea SA WC21.1L L. pauciflora SA XHRla $L$. simsiana SA Betal106f $L$. pungens SA $R$. palustris (ATCC17001) $M$. nodulans (ORS2060) Az. caulinodans (LMG6465)

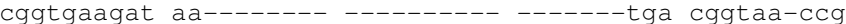
cggtgaagat aa-------- --------- -------tga cggtaa-ccg gcgggaagat aa-------- ---------- -------tga cggtac-cgc

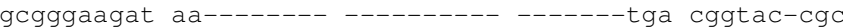
gcgggaagat aa-------- ---------- -------tga cggtac-cgc

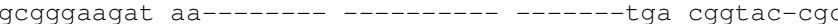

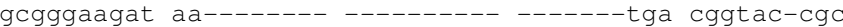
gcgggaagat aa-------- --------- -------tga cggtac-cgc gcgggaagat aa-------- --------- -------tga cggtac-cgc gcgggaagat aa-------- ---------- -------tga cggtac-cgc gcgggaagat aa-------- ---------- -------tga cggtac-cgc

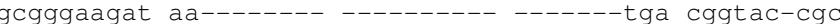
tcgggacgat aa-------- --------- -------tga cggtac-cgg cggtgaagat aa-------- --------- -------tga cggtaa-ccg

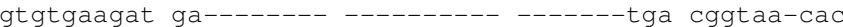
ccacgacgat ga-------- ---------- -------tga cggtag-tgg acgcgacgat ga-------- --------- -------tga cggtag-cgt acgcgacgat ga-------- --------- -------tga cggtag-cgt ccacgacgat aa-------- --------- -------tga cggtag-tgg

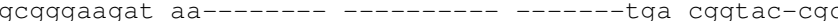
cggtgaagat aa-------- ---------- ------ttga cggtaa-ccg cggtgaagat aa-------- ---------- -------tga cggtaa-ccg

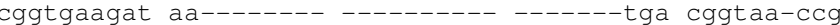
cggtgaagat aa-------- --------- -------tga cggtaa-ccg cggtgaagat aa-------- --------- -------tga cggtaa-ccg cggtgaagat aa-------- --------- -------tga cggtaa-ccg gcgggaagat aa-------- ---------- -------tga cggtac-cgc gcgggaagat aa-------- --------- -------tga cggtac-cgc gcgggaagat aa-------- --------- -------tga cggtac-cgc cggtgaagat aa-------- --------- -------tga cggtaa-ccg

460

470

490

500

tagaagaagc cccggctaac ttcgtgccag cagccgcggt aatacg-aatagaagaagc cccggctaac ttcgtgccag cagccgcggt aatacg-aatagaagaagc cccggctaac ttcgtgccag cagccgcggt aatacg-aagagaagaagc cccggctaac ttcgtgccag cagccgcggt aatacg-aatagaagaagc cccggctaac ttcgtgccag cagccgcggt aatacg-aatagaagaagc cccggctaac ttcgtgccag cagccgcggt aatacg-aatagaagaagc cccggctaac ttcgtgccag cagccgcggt aatacg-aatagaagaagc cccggctaac ttcgtgccag cagccgcggt aatacg-aatagaagaagc cccggctaac ttcgtgccag cagccgcggt aatacgaaatagaagaagc cccggctaac ttcgtgccag cagccgcggt aatacg-aatagaagaagc cccggctaac ttcgtgccag cagccgcggt aatacg-aatagaagaagc cccggctaac ttcgtgccag cagccgcggt aatacg-aatagaagaagc cccggctaac ttcgtgccag cagccgcggt aatacg-aatagaagaagc cccggctaac ttcgtgccag cagccgcggt aatacg-aatagaagaagc cccggctaac ttcgtgccag cagccgcggt aatacg-aatagaagaagc cccggctaac ttcgtgccag cagccgcggt aatacg-aatagaagaagc cccggctaac ttcgtgccag cagccgcggt aatacg-aatagaagaagc cccggctaac ttcgtgccag cagccgcggt aatacg-aatagaagaagc cccggctaac ttcgtgccag cagccgcggt aatacg-aagagaagaagc cccggctaac ttcgtgccag cagccgcggt aatacg-aagagaagaagc cccggctaac ttcgtgccag cagccgcggt aatacg-aagagaagaagc cccggctaac ttcgtgccag cagccgcggt aatacg-aagagaagaagc cccggctaac ttcgtgccag cagccgcggt aatacg-aagagaagaagc cccggctaac ttcgtgccag cagccgcggt aatacg-aagagaagaagc cccggctaac ttcgtgccag cagccgcggt aatacg-aagagaagaagc cccggctaac ttcgtgccag cagccgcggt aatacg-aag gagaagaagc cccggctaac ttcgtgccag cagccgcggt aatacg-aagagaagaagc cccggctaac ttcgtgccag cagccgcggt aatacg-aagagaagaagc cccggctaac ttcgtgccag cagccgcggt aatacg-aagagaagaagc cccggctaac ttcgtgccag cagccgcggt aatacg-aagagaagaagc cccggctaac ttcgtgccag cagccgcggt aatacg-aagagaagaagc cccggctaac ttcgtgccag cagccgcggt aatacg-aagagaagaagc cccggctaac ttcgtgccag cagccgcggt aatacg-aaaagaataagc cccggctaac ttcgtgccag cagccgcggt aatacg-aaaagaataagc cccggctaac ttcgtgccag cagccgcggt aatacg-aaaagaataagc cccggctaac ttcgtgccag cagccgcggt aatacg-aaaagaataagc cccggctaac ttcgtgccag cagccgcggt aatacg-aaaagaataagc cccggctaac ttcgtgccag cagccgcggt aatacg-aaaagaataagc cccggctaac ttcgtgccag cagccgcggt aatacg-aaaagaataagc cccggctaac ttcgtgccag cagccgcggt aatacg-aaaagaataagc cccggctaac ttcgtgccag cagccgcggt aatacg-aaaagaataagc cccggctaac ttcgtgccag cagccgcggt aatacg-aaaagaataagc cccggctaac ttcgtgccag cagccgcggt aatacg-aaaggaataagc cccggctaac ttcgtgccag cagccgcggt aatacg-aagagaagaagc cccggctaac ttcgtgccag cagccgcggt aatacg-aa- 
R. rubrum (LMGATCC11170)

A. magnetotacticum (M58171)

Az. brasilense (NCIMB11860)

Az. lipoferum (NCIMB11861)

R. acidophila (M34128)

A. clevelandensis (M69186)

$S$. morelense (LMG21331) T

M. amorphae (LMG18977) T

$M$. ciceri (LMG14989) T

$M$. huakuii (LMG14107) T

M. loti (LMG6125) T

M. plurifarium (LMG11892) T

B. elkanii (LMG6134)T

B. beta (LMG21987) T

B. yuanmingense (CCBAU10071) T

S. medicae (A321) T

M. mediterraneum (LMG17148) T

$M$. septentrionale (SDW014)T

$M$. temperatum (SDW018) T

$M$. thiogangeticum (SJT) T

M.tianshanense (A-1BS) T

WC12.1a L. sepiaria SA

WC19.1b $L$. cytisoides SA

WC19.1c L. cytisoides SA

WC23.1b $L$. multiflora SA

WC23.1c $L$. multiflora SA

WC23.1d L. multiflora SA

WC28.1F L. sericea SA

WC28.1g $L$. sericea SA

WC28.1h $L$. sericea SA

WC28.1i L. sericea SA

WC33b $L$. sericea SA

WC33c $L$. sericea SA

WC33e L. sericea SA

WC33h $L$. sericea SA

S. americanum (CFNEI156) T

S. arboris (LMG14919) T

S. fredii (LMG6217) T

S. kostiense (LMG19227)

S. kummerowiae (CCBAU71714) T

$S$. meliloti (LMG6133) T

BH1LSC L. spinescens SA

BH2LSa $L$. spinescens SA

BH3LSd $L$. spinescens SA

BW1LSab $L$. spinescens SA

BW2LSa $L$. spinescens SA

BW2LSb $L$. spinescens SA

BW2LSC $L$. spinescens SA

BW3LSd $L$. spinescens SA

$B$. canariense (MSFC2-M3-R-3) T

B. japonicum (LMG6138) T

B. liaoningense (LMG18230) T

Betallo6d L. pungens SA

Betal106a L. pungens SA

NK22 $L$. sericea SA

WC21.1L $L$. pauciflora SA

XHR1a L. simsiana SA

Betal106f L. pungens SA

$R$. palustris (ATCC17001)

$M$. nodulans (ORS2060)

Az. caulinodans (LMG6465)

$R$. rubrum (LMGATCC11170)

A. magnetotacticum (M58171)

$A z$. brasilense (NCIMB11860)

$A z$. lipoferum (NCIMB11861)

R. acidophila (M34128)

A. clevelandensis (M69186)

$S$. morelense (LMG21331) T

$M$. amorphae (LMG18977) T

M. ciceri (LMG14989) T

M. huakuii (LMG14107)T

M. loti (LMG6125)T

M. plurifarium (LMG11892) T

B. elkanii (LMG6134)T

B. beta (LMG21987) T

cagaagaagc cccggctaac ttcgtgccag cagccgcggt aatacg-aagagaagaagc cccggctaac ttcgtgccag cagccgcggt aatacg-aagagaagaagc cccggctaac ttcgtgccag cagccgcggt aatacg-aagagaagaagc cccggctaac ttcgtgccag cagccgcggt aatacg-aagcgaagaagc cccggctaac ttcgtgccag cagccgcggt aatacg-aaaagaataagc cccggctaac ttcgtgccag cagccgcggt aatacg-aagagaagaagc cccggctaac ttcgtgccag cagccgcggt aatacg-aatagaagaagc cccggctaac ttcgtgccag cagccgcggt aatacg-aatagaagaagc cccggctaac ttcgtgccag cagccgcggt aatacg-aatagaagaagc cccggctaac ttcgtgccag cagccgcggt aatacg-aatagaagaagc cccggctaac ttcgtgccag cagccgcggt aatacg-aatagaagaagc cccggctaac ttcgtgccag cagccgcggt aatacg-aaaagaataagc cccggctaac ttcgtgccag cagccgcggt aatacg-aaaagaataagc cccggctaac ttcgtgccag cagccgcggt aatacg-aaaagaataagc cccggctaac ttcgtgccag cagccgcggt aatacg-aagagaagaagc cccggctaac ttcgtgccag cagccgcggt aatacg-aa-

510

520

530

540

gggggctagc gttgttcgga attactgggc gtaaagcgc- --acgtaggc gggggctagc gttgttcgga attactgggc gtaaagcgc- --acgtaggc gggggctagc gttgttcgga attactgggc gtaaagcgc- --acgtaggc gggggctagc gttgttcgga attactgggc gtaaagcgc- --acgtaggc gggggctagc gttgttcgga attactgggc gtaaagcgc- --acgtaggc gggggctagc gttgttcgga attactgggc gtaaagcgc- --acgtaggc gggggctagc gttgttcgga attactgggc gtaaagcgc- --acgtaggc gggggctagc gttgttcgga attactgggc gtaaagcgc- --acgtaggc gggggctagc gttgttcgga attactgggc gtaaagcgc- --acgtaggc gggggctagc gttgttcgga attactgggc gtaaagcgc- --acgtaggc gggggctagc gttgttcgga attactgggc gtaaagcgc- --acgtaggc gggggctagc gttgttcgga attactgggc gtaaagcgc- --acgtaggc gggggctagc gttgttcgga attactgggc gtaaagcgc- --acgtaggc gggggctagc gttgttcgga attactgggc gtaaagcgc- --acgtaggc gggggctagc gttgttcgga attactgggc gtaaagcgc- --acgtaggc gggggctagc gttgttcgga attactgggc gtaaagcgc- --acgtaggc gggggctagc gttgttcgga attactgggc gtaaagcgc- --acgtaggc gggggctagc gttgttcgga attactgggc gtaaagcgc- --acgtaggc gggggctagc gttgttcgga attactgggc gtaaagcgc- --acgtaggc gggggctagc gttgttcgga attactgggc gtaaagcgc- --acgtaggc gggggctagc gttgttcgga attactgggc gtaaagcgc- --acgtaggc gggggctagc gttgttcgga attactgggc gtaaagcgc- --acgtaggc gggggctagc gttgttcgga attactgggc gtaaagcgc- --acgtaggc gggggctagc gttgttcgga attactgggc gtaaagcgc- --acgtaggc gggggctagc gttgttcgga attactgggc gtaaagcgc- --acgtaggc gggggctagc gttgttcgga attactgggc gtaaagcgc- --acgtaggc gggggctagc gttgttcgga attactgggc gtaaagcgc- --acgtaggc gggggctagc gttgttcgga attactgggc gtaaagcgc- --acgtaggc gggggctagc gttgttcgga attactgggc gtaaagcgc- --acgtaggc gggggctagc gttgttcgga attactgggc gtaaagcgc- --acgtaggc gggggctagc gttgttcgga attactgggc gtaaagcgc- --acgtaggc gggggctagc gttgttcgga attactgggc gtaaagcgc- --acgtaggc gggggctagc gttgttcgga attactgggc gtaaagcgc- --acgtaggc gggggctagc gttgctcgga atcactgggc gtaaagggt- --gcgtaggc gggggctagc gttgctcgga atcactgggc gtaaagggt- --gcgtaggc gggggctagc gttgctcgga atcactgggc gtaaagggt- --gcgtaggc gggggctagc gttgctcgga atcactgggc gtaaagggt- --gcgtaggc gggggctagc gttgctcgga atcactgggc gtaaagggt- --gcgtaggc gggggctagc gttgctcgga atcactgggc gtaaagggt- --gcgtaggc gggggctagc gttgctcgga atcactgggc gtaaagggt- --gcgtaggc gggggctagc gttgctcgga atcactgggc gtaaagggt- --gcgtaggc gggggctagc gttgctcgga atcactgggc gtaaagggtt gcgtaaggcg gggggctagc gttgctcgga atcactgggc gtaaagggt- --gcgtaggc gggggctagc gttgctcgga atcactgggc gtaaagggc- --gcgtaggc gggggcaagc gttgctcgga atcactgggc gtaaagcgc- --acgtaggc gggggcaagc gttgttcgga attactgggc gtaaagagc- --gcgtaggc gggggctagc gttgttcgga attactgggc gtaaagcgc- --acgcaggc gggggcgagc gttgttcgga attactgggc gtaaagggc- --gcgtaggc gggggctagc gttgttcgga attactgggc gtaaagggc- --gcgtaggc gggggc-agc gttgttcgga tttactgggc gtaaagcgc- --acgtaggc gggggctagc gttgctcgga atctctgggc gtaaagggt- --gcgtaggc gggggctagc gttgttcgga attactgggc gtaaagcgc- --acgtaggc gggggctagc gttgttcgga attactgggc gtaaagcgc- --acgtaggc gggggctagc gttgttcgga attactgggc gtaaagcgc- --acgtaggc gggggctagc gttgttcgga attactgggc gtaaagcgc- --acgtaggc gggggctagc gttgttcgga attactgggc gtaaagcgc- --acgtaggc gggggctagc gttgttcgga attactgggc gtaaagcgc- --acgtaggc gggggctagc gttgctcgga atcactgggc gtaaagggt- --gcgtaggc gggggctagc gttgctcgga atcactgggc gtaaagggt- --gcgtaggc 
B. yuanmingense (CCBAU10071) T S. medicae (A321) T

M. mediterraneum (LMG17148) T

$M$. septentrionale (SDW014) T

$M$. temperatum (SDW018) T

$M$. thiogangeticum (SJT) $T$

$M$. tianshanense (A-1BS) T

WC12.1a $L$. sepiaria SA

WC19.1b $L$. cytisoides SA

WC19.1c $L$. cytisoides SA

WC23.1b L. multiflora SA

WC23.1c L. multiflora SA

WC23.1d $L$. multiflora SA

WC28.1F $L$. sericea SA

WC28.1g $L$. sericea SA

WC28.1h $L$. sericea SA

WC28.1i $L$. sericea SA

WC33b L. sericea SA

WC33c $L$. sericea SA

WC33e $L$. sericea SA

WC33h I sericea SA

S. americanum (CFNEI156) T

$S$. arboris (LMG14919) T

S. fredii (LMG6217) T

S. kostiense (LMG19227)

S. kummerowiae (CCBAU71714) T

S. meliloti (LMG6133) T

BH1LSC $L$. spinescens SA

BH2LSa $L$. spinescens SA

BH3LSd $L$. spinescens SA

BW1LSab $L$. spinescens SA

BW2LSa $L$. spinescens SA

BW2LSb $L$. spinescens SA

BW2LSC $L$. spinescens SA

BW3LSd $L$. spinescens SA

$B$. canariense (MSFC2-M3-R-3) T

B. japonicum (LMG6138) T

B. liaoningense (LMG18230) T

Betallo6d L. pungens SA

Betal106a $L$. pungens SA

NK22 $L$. sericea SA

WC21.1L L. pauciflora SA

XHRla $L$. simsiana SA

Betal106f $L$. pungens SA

$R$. palustris (ATCC17001)

$M$. nodulans (ORS2060)

Az. Caulinodans (LMG6465)

$R$. rubrum (LMGATCC11170)

A. magnetotacticum (M58171)

Az. brasilense (NCIMB11860)

$A z$. lipoferum (NCIMB11861)

$R$. acidophila (M34128)

A. clevelandensis (M69186)

S. morelense (LMG21331) T

$M$. amorphae (LMG18977) T

M. ciceri (LMG14989) T

M. huakuii (LMG14107) T

M. Loti (LMG6125) T

M. plurifarium (LMG11892) T

B. elkanii (LMG6134) T

B. beta (LMG21987) T

B. yuanmingense (CCBAU10071) T

S. medicae (A321) T

M. mediterraneum (LMG17148) T

$M$. septentrionale (SDW014) T

$M$. temperatum (SDW018) T

$M$. thiogangeticum (SJT) T

$M$.tianshanense (A-1BS) T

WC12.1a $L$. sepiaria SA

WC19.1b L. cytisoides SA

WC19.1c $L$. cytisoides SA

WC23.1b $L$. multiflora SA

WC23.1C L. multiflora SA

gggggctagc gttgctcgga atcactgggc gtaaagggt- --gcgtaggc gggggctagc gttgttcgga attactgggc gtaaagcgc- --acgtaggc

560

570

580

590 ggatatttaa gtcagggg-t gaaa-tcccg gggctcaac- cccgg-aact ggactattaa gtcagggg-t gaaa-tcccg gggctcaac- cccgg-aact ggatatttaa gtcagggg-t gaaa-tcccg gggctcaac- cccgg-aact ggatacttaa gtcagggg-t gaaa-tcccg gggctcaac- cccgg-aact ggatacttaa gtcagggg-t gaaa-tcccg gggctcaac- cccgg-aact ggatacttaa gtcagggg-t gaaa-tcccg gggctcaac- cccgg-aact ggatacttaa gtcagggg-t gaaa-tcccg gggctcaac- cccgg-aact ggatacttaa gtcagggg-t gaaa-tcccg gggctcaac- cccgg-aact ggatacttaa gtcagggg-t gaaa-tcccg gggctcaac- cccgg-aact ggatacttaa gtcagggg-t gaaa-tcccg gggctcaac- cccgg-aact ggattgttaa gttagggg-t gaaa-tcccg gggctcaac- cccgg-aact ggatacttaa gtcagggg-t gaaa-tcccg gggctcaac- cccgg-aact ggatacttaa gtcaggggtt gaaa-tcccg gggctcaac- cccgg-aact ggattgttaa gttagggg-t gaaa-tcccg gggctcaac- cccgg-aact ggattgttaa gttagggg-t gaaa-tcccg gggctcaac- cccgg-aact ggatacttaa gtcagggg-t gaaa-tcccg gggctcaac- cccgg-aact ggattgttaa gttaggggtt gaaa-tcccg gggctcaac- cccgg-aact ggacatttaa gtcagggg-t gaaa-tcccg gggctcaac- cccgg-aact ggattgttaa gtgagggg-t gaaa-tccca gggctcaac- cctgg-aact ggacatttaa gtcagggg-t gaaa-tcccg gggctcaac- cccgg-aact ggacatttaa gtcagggg-t gaaa-tcccg gggctcaac- cccgg-aact ggacatttaa gtcagggg-t gaaa-tcccg gggctcaac- cccgg-aact ggattgttaa gtgagggg-t gaaa-tccca gggctcaac- cctgg-aact ggattgttaa gtgagggg-t gaaa-tccca gggctcaac- cctgg-aact ggattgttaa gtgagggg-t gaaa-tccca gggctcaac- cctgg-aact ggattgttaa gtgagggg-t gaaa-tccca gggctcaac- cctgg-aact ggattgttaa gtgagggg-t gaaa-tccca gggctcaac- cctgg-aact ggattgttaa gtgagggg-t gaaa-tccca gggctcaac- cctgg-aact ggattgttaa gtgagggg-t gaaa-tccca gggctcaac- cctgg-aact ggattgttaa gtgagggg-t gaaa-tccca gggctcaac- cctgg-aact ggattgttaa gtgagggg-t gaaa-tccca gggctcaac- cctgg-aact gggtctttaa gtcagggg-t gaaa-tcctg gagctcaac- tccagaaact gggtctttaa gtcagggg-t gaaa-tcctg gagctcaac- tccag-aact gggtctttaa gtcagggg-t gaaa-tcctg gagctcaac- tccag-aact gggtctttaa gtcagggg-t gaaa-tcctg gagctcaac- tccag-aact gggtctttaa gtcagggg-t gaaa-tcctg gagctcaac- tccag-aact gggtctttaa gtcagggg-t gaaa-tcctg gagctcaac- tccag-aact gggtctttaa gtcagggg-t gaaa-tcctg gagctcaac- tccag-aact gggtttttaa gtcagggg-t gaaa-tcctg gagctcaac- tccag-aact ggtcttttaa gtcagggg-t gaaattcctg gagctcaact tccag-aact gggtttctaa gtcagagg-t gaaa-gcctg gagctcaac- tccag-aact ggctcgccaa gtcggggg-t gaaa-gcccg tggctcaac- cacgg-aatg ggatcgttaa gtcagggg-t gaaa-gcctg gagctcaac- tccag-aact ggtctgatta gtcagagg-t gaaa-tccca gagctcaac- tttgg-aact ggtggtcata gtcagaag-t gaaa-gccct gggctcaac- ccggg-aatt ggcccgatca gtcagatg-t gaaa-gcccc gggctcaac- ctggg-aact ggcccgttta gtcagaag-t gaaa-gcccc gggctcaac- ctggg-aaca - gatctttaa gtcagggg-t gaaa-tgccg gagctcaac- ttcgg-aact gggtctttaa gtcagagg-t gaaa-gcctg gagctcaac- tccag-aact ggacatttaa gtcagggg-t gaaa-tccca gagctcaac- tctgg-aact ggatacttaa gtcagggg-t gaaa-tcccg gggctcaac- cccgg-aact ggattgttaa gttagggg-t gaaa-tccca gggctcaac- cctgg-aact ggatacttaa gtcagggg-t gaaa-tcccg gggctcaac- cccgg-aact ggattgttaa gttagggg-t gaaa-tccca gggctcaac- cctgg-aact ggatacttaa gtcagggg-t gaaa-tcccg gggctcaac- cccgg-aact gggtctttaa gtcagggg-t gaaa-tcctg gagctcaac- tccag-aact gggtctttaa gtcagggg-t gaaa-tcctg gagctcaac- tccag-aact gggtctttaa gtcagggg-t gaaa-tcctg gagctcaac- tccag-aact ggattgttaa gtgagggg-t gaaa-tccca gggctcaac- cctgg-aact

610

620

630

640

650 gccttgata ctggg-tatc tcgagtccga g--aga-ggt gagtgg-aat gcctttgata ctggg-tatc tcgagtccgg a--aga-ggt gagtgg-aat gcctttgata ctggg-tatc tcgagtccga g--aga-ggt gagtgg-aat gcctttgata ctggt-ggtc tagagtccgg a--aga-ggt gagtgg-aat gcctttgata ctggg-tatc tcgagtccga g--aga-ggt gagtgg-aat gccttgata ctggg-tatc tcgagtccgg a--aga-ggt gagtgg-aat gcctttgata ctggg-tatc tcgagtccgg a--aga-ggt gagtgg-aat gcctttgata ctggg-tatc tcgagtccgg a--aga-ggt gagtgg-aat gcctttgata ctggg-tatc tcgagtccgg a--aga-ggt gagtgg-aat gcctttgata ctggg-tatc tcgagtccgg a--aga-ggt gagtgg-aat 
WC23.1d $L$. multiflora SA WC28.1F $L$. sericea SA WC28.1g $L$. sericea SA WC28.1h $L$. sericea SA WC28.1i L. sericea SA WC33b L. sericea SA WC33C $L$. sericea SA WC33e L. sericea SA WC33h L. sericea SA

$S$. americanum (CFNEI156) T

$S$. arboris (LMG14919) T

S. fredii (LMG6217) T

S. kostiense (LMG19227)

S. kummerowiae (CCBAU71714) T

S. meliloti (LMG6133) T

BH1LSC $L$. spinescens SA

BH2LSa $L$. spinescens SA

BH3LSd $L$. spinescens SA

BW1LSab $L$. spinescens SA

BW2LSa $L$. spinescens SA

BW2LSb $L$. spinescens SA

BW2LSC $L$. spinescens SA

BW3LSd $L$. spinescens SA

B. canariense (MSFC2-M3-R-3) T

$B$. japonicum (LMG6138) T

$B$. liaoningense (LMG18230) T

Betal106d $L$. pungens SA

Betal106a $L$. pungens SA

NK22 $L$. sericea SA

WC21.1L $L$. pauciflora SA

XHRla $L$. simsiana SA

Betal106f $L$. pungens SA

$R$. palustris (ATCC17001)

$M$. nodulans (ORS2060)

Az. caulinodans (LMG6465)

R. rubrum (LMGATCC11170)

A. magnetotacticum (M58171)

Az. brasilense (NCIMB11860)

Az. Iipoferum (NCIMB11861)

R. acidophila (M34128)

A. clevelandensis (M69186)

$S$. morelense (LMG21331) T

$M$. amorphae (LMG18977) T

M. ciceri (LMG14989) T

$M$. huakuii (LMG14107) T

M. loti (LMG6125) T

M. plurifarium (LMG11892) T

B. elkanii (LMG6134) T

B. beta (LMG21987) T

B. yuanmingense (CCBAU10071) T

S. medicae (A321) T

M. mediterraneum (LMG17148) T

$M$. septentrionale (SDW014) T

M. temperatum (SDW018) T

$M$. thiogangeticum (SJT) T

$M . t i a n s h a n e n s e$ (A-1BS) T

WC12.1a L. sepiaria SA

WC19.1b L. cytisoides SA

WC19.1C $L$. cytisoides SA

WC23.1b $L$. multiflora SA

WC23.1C $L$. multiflora SA

WC23.1d I multiflora SA

WC28.1F $L$. sericea SA

WC28.1g L. sericea SA

WC28.1h $L$. sericea SA

WC28.1i $L$. sericea SA

WC33b L. sericea SA

WC33c $L$. sericea SA

WC33e $L$. sericea SA

WC33h $L$. sericea SA

S. americanum (CFNEI156) T

S. arboris (LMG14919) T

S. fredii (LMG6217) T

S. kostiense (LMG19227)

S. kummerowiae (CCBAU71714) T gcctttgata ctggg-tatc tcgagtccgg a--aga-ggt gagtgg-aat gcctttgata ctggg-tatc tcgagtccgg a--aga-ggt gagtgg-aat gccttaata ctggc-aatc tcgagtccga g--aga-ggt gagtgg-aat gcctttgata ctggg-tatc tcgagtccgg a--aga-ggt gagtgg-aat gcctttgata ctggg-tatc tcgagtccgg a--aga-ggt gagtgg-aat gccttaata ctggc-aatc tcgagtccga g--aga-ggt gagtgg-aat gccttaata ctggc-aatc tcgagtccga g--aga-ggt gagtgg-aat gcctttgata ctggg-tatc tcgagtccgg a--agagggt gagtgg-aat gccttaata ctggc-aatc tcgagtccga g--aga-ggt gagtggaaat gccttgata ctggg-tgtc tagagtccgg a--aga-ggt gagtgg-aat gccttcata ctggc-aatc tagagtccag a--aga-ggt gagtgg-aat gcctttgata ctggg-tgtc tagagtccgg a--aga-ggt gagtgg-aat gcctttgata ctggg-tgtc tagagtccgg a--aga-ggt gagtgg-aat gcctttgata ctggg-tgtc tagagtccgg a--aga-ggt gagtgg-aat gccttcata ctggc-aatc tagagtccag a--aga-ggt gagtgg-aat gccttcata ctggc-aatc tagagtccag a--aga-ggt gagtgg-aat gccttcata ctggc-aatc tagagtccag a--aga-ggt gagtgg-aat gcctttcata ctggc-aatc tagagtccag a--aga-ggt gagtgg-aat gccttcata ctggc-aatc tagagtccag a--aga-ggt gagtgg-aat gccttcata ctggc-aatc tagagtccag a--aga-ggt gagtgg-aat gccttcata ctggc-aatc tagagtccag a--aga-ggt gagtgg-aat gccttcata ctggc-aatc tagagtccag a--aga-ggt gagtgg-aat gcctttcata ctggc-aatc tagagtccag a--aga-ggt gagtgg-aat gccttgata ctgaa-gatc ttgagttcgg g--aga-ggt gagtgg-aac gcctttgata ctgag-gatc ttgagttcgg g--aga-ggt gagtgg-aac gcctttgata ctgaa-gatc ttgagttcgg g--aga-ggt gagtgg-aac gccttgata ctgaa-gatc ttgagttcgg g--aga-ggt gagtgg-aac gcctttgata ctgag-gatc ttgagttcgg g--aga-ggt gagtgg-aac gccttgata ctgaa-gatc ttgagttcgg g--aga-ggt gagtgg-aac gccttgata ctgaa-gatc ttgagttcgg g--aga-ggt gagtgg-aac gcctttgata ctgaa-gatc ttgagtccgg g--aga-ggt gagtgg-aac gccttgata ctgaaggatc ttgagttcgg ggagaa-ggt gagtgggaac gccttgata ctgga-agtc ttgagtatgg c--aga-ggt gagtgg-aac gccttcgata ctggc-gggc ttgagaccgg a--aga-gga cagcgg-aac gccctgata ctggc-gatc ttgagttcga g--aga-ggt tggtgg-aac gcctttgata ctgtt-agac tagaatccgt g--aga-ggg tggtgg-aat gcttttgata ctgga-ccgc tagaatcacg g--aga-ggg tagtgg-aat gcatttgata ctgtc-gggc ttgagttccg g--aga-gga tggtgg-aat gcttttgata ctggc-gggc ttgagttccg g--aga-gga tggtgg-aat gcctttgata ctgga-gatc ttgagttcga g--aga-ggt gagtgg-aac gccttgata ctgag-gatc ttgagttcgg g--aga-ggt gagtgg-aac gcctttgata ctggg-tgtc tagagtatgg a--aga-ggt gagtgg-aat gcctttgata ctggg-tatc tcgagtccgg a--aga-ggt gagtgg-aat gccttaata ctggc-aatc tcgagtccga g--aga-ggt gagtgg-aat gcctttgata ctggg-tatc tcgagtccgg a--aga-ggt gagtgg-aat gccttaata ctggc-aatc tcgagtccga g--aga-ggt gagtgg-aat gcctttgata ctggg-tatc tggagtccgg a--aga-ggt gagtgg-aat gcctttgata ctgaa-gatc ttgagttcgg g--aga-ggt gagtgg-aac gcctttgata ctgaa-gatc ttgagttcgg g--aga-ggt gagtgg-aac gcctttgata ctgaa-gatc ttgagtccgg g--aga-ggt gagtgg-aac gcctttcata ctggc-aatc tagagtccag a--aga-ggt gagtgg-aat

660

680

tccgagtgta gaggtgaat tcgtagatat tcgg--agga acaccagtgg tccgagtgta gaggtgaaat tcgtagatat tcgg--agga acaccagtgg tccgagtgta gaggtgaaat tcgtagatat tcgg--agga acaccagtgg tccgagtgta gaggtgaaat tcgtagatat tcgg--agga acaccagtgg tccgagtgta gaggtgaaat tcgtagatat tcgg--agga acaccagtgg tccgagtgta gaggtgaaat tcgtagatat tcgg--agga acaccagtgg tccgagtgta gaggtgaaat tcgtagatat tcgg--agga acaccagtgg tccoagtgta gaggtgaaat tcgtagatat tcgg--agga acaccagtgg tccgagtgta gaggtgaaat tcgtagatat tcgg--agga acaccagtgg tccgagtgta gaggtgaaat tcgtagatat tcgg--agga acaccagtgg tccgagtgta gaggtgaaat tcgtagatat tcgg--agga acaccagtgg tccgagtgta gaggtgaaat tcgtagatat tcgg--agga acaccagtgg tccgagtgta gaggtgaaat tcgtagatat tcgg--agga acaccagtgg tccgagtgta gaggtgaaat tcgtagatat tcgg--agga acaccagtgg tccgagtgta gaggtgaaat tcgtagatat tcgg--agga acaccagtgg tccgagtgta gaggtgaaat tcgtagatat tcgg--agga acaccagtgg tccgagtgta gaggtgaaat tcgtagatat tcgg--agga acaccagtgg tccgagtgta gaggtgaaat tcgtagatat tcgggaggaa acaccagtgg tccgagtgta gaggtgaaat tcgtagatat tcgg--agga acaccagtgg tccgagtgta gaggtgaaat tcgtagatat tcgg--agga acaccagtgg tccgagtgta gaggtgaaat tcgtagatat tcgg--agga acaccagtgg tccgagtgta gaggtgaaat tcgtagatat tcgg--agga acaccagtgg tccgagtgta gaggtgaat tcgtagatat tcgg--agga acaccagtgg tccgagtgta gaggtgaaat tcgtagatat tcgg--agga acaccagtgg 
S. meliloti (LMG6133) T BH1LSC $L$. spinescens SA BH2LSa $L$. spinescens SA BH3LSd $L$. spinescens SA BW1LSab $L$. spinescens SA BW2LSa $L$. spinescens SA BW2LSb $L$. spinescens SA BW2LSC $L$. spinescens SA BW3LSd L. spinescens SA

$B$. canariense (MSFC2-M3-R-3) T

$B$. japonicum (LMG6138) T

B. liaoningense (LMG18230) T

Betal106d $L$. pungens SA

Betal106a L. punges SA

NK22 $L$. sericea SA

WC21.1L L. pauciflora SA

XHRla $L$. simsiana SA

Betal106f $L$. pungens SA

$R$. palustris (ATCC17001)

$M$. nodulans (ORS2060)

Az. caulinodans (LMG6465)

$R$. rubrum (LMGATCC11170)

A. magnetotacticum (M58171)

Az. brasilense (NCIMB11860)

$A z$. lipoferum (NCIMB11861)

R. acidophila (M34128)

A. clevelandensis (M69186)

S. morelense (LMG21331) T

$M$. amorphae (LMG18977) T

M. ciceri (LMG14989) T

$M$. huakuii (LMG14107) T

M. Loti (LMG6125) T

M. plurifarium (LMG11892)T

B. elkanii (LMG6134) T

B. beta (LMG21987) T

B. yuanmingense (CCBAU10071) T

S. medicae (A321) T

M. mediterraneum (LMG17148)T

$M$. septentrionale (SDW014) T

$M$. temperatum (SDW018) T

$M$. thiogangeticum (SJT) T

$M . t i a n s h a n e n s e$ (A-1BS) T

WC12.1a $L$. sepiaria SA

WC19.1b $L$. cytisoides SA

WC19.1C $L$. cytisoides SA

WC23.1b $L$. multiflora SA

WC23.1C L. multiflora SA

WC23.1d L. multiflora SA

WC28.1F $L$. sericea SA

WC28.1g $L$. sericea SA

WC28.1h $L$ sericea SA

WC28.1i $L$. sericea SA

WC33b $L$. sericea SA

WC33C $L$. sericea SA

WC33e $L$. sericea SA

WC33h $L$. sericea SA

$S$. americanum (CFNEI156) T

$S$. arboris (LMG14919) T

S. fredii (LMG6217) T

S. kostiense (LMG19227)

S. kummerowiae (CCBAU71714) T

S. meliloti (LMG6133) T

BH1LSc $L$. spinescens SA BH2LSa $L$. spinescens SA BH3LSd $L$. spinescens SA BW1LSab $L$. spinescens SA BW2LSa $L$. spinescens SA BW2LSb $L$. spinescens SA BW2LSC $L$. spinescens SA BW3LSd $L$. spinescens SA

$B$. canariense (MSFC2-M3-R-3) T

B. japonicum (LMG6138) T

$B$. liaoningense (LMG18230) T

Betall06d L. pungens SA

Betal106a L. pungens SA tccgagtgta gaggtgaaat tcgtagatat tcgg--agga acaccagtgg tccgagtgta gaggtgaat tcgtagatat tcgg--agga acaccagtgg tccgagtgta gaggtgaaat tcgtagatat tcgg--agga acaccagtgg tccgagtgta gaggtgaaat tcgtagatat tcgg--agga acaccagtgg tccgagtgta gaggtgaat tcgtagatat tcgg--agga acaccagtgg tccgagtgta gaggtgaaat tcgtagatat tcgg--agga acaccagtgg tccgagtgta gaggtgaaat tcgtagatat tcgg--agga acaccagtgg tccgagtgta gaggtgaat tcgtagatat tcgg--agga acaccagtgg tccgagtgta gaggtgaaat tcgtagatat tcgg--agga acaccagtgg tgcgagtgta gaggtgaaat tcgtagatat tcgc--aaga acaccagtgg tgcgagtgta gaggtgaaat tcgtagatat tcgc--aaga acaccagtgg tgcgagtgta gaggtgaaat tcgtagatat tcgc--aaga acaccagtgg tgcgagtgta gaggtgaaat tcgtagatat tcgc--aaga acaccagtgg tgcgagtgta gaggtgaaat tcgtagatat tcgc--aaga acaccagtgg tgcgagtgta gaggtgaat tcgtagatat tcgc--aaga acaccagtgg tgcgagtgta gaggtgaaat tcgtagatat tcgc--aaga acaccagtgg tgcgagtgta gaggtgaaat tcgtagatat tcgc--aaga acaccagtgg kgcgagtgta gaggtgaaat tcgtagatat tcgc--aaga acaccagtgg tgcgagtgta gaggtgaaat tcgtagatat tcgc--aaga acaccagtgg tgcgagtgta gaggtgaaat tcgtagatat tcgc--aaga acaccagtgo tccgagtgta gaggtgaat tcgtagatat tcgg--aaga acaccagtgg tcccagtgta gaggtgaaat tcgtagatat tggg--agga acaccagtgg tccgagtgta gaggtgaaat tcgtagatat tcgg--aaga acaccagtgg tcccagtgta gaggtgaaat tcgtagatat tggg--aaga acaccggtgg tcccagtgta gaggtgaaat tcgtagatat tggg--aaga acaccggtgg tgcgagtgta gaggtgaaat tcgtagatat tcgc--aaga acaccagtgg tgcgagtgta gaggtgaaat tcgtagatat tcgc--aaga acaccagtgg tccgagtgta gaggtgaaat tcgtagatat tcgg--agga acaccagtgg tccgagtgta gaggtgaaat tcgtagatat tcgg--agga acaccagtgg tccgagtgta gaggtgaaat tcgtagatat tcgg--agga acaccagtgg tccgagtgta gaggtgaaat tcgtagatat tcgg--agga acaccagtgg tccgagtgta gaggtgaaat tcgtagatat tcgg--agga acaccagtgg tccgagtgta gaggtgaaat tcgtagatat tcgg--agga acaccagtgg tgcgagtgta gaggtgaaat tcgtagatat tcgc--aaga acaccagtgg tgcgagtgta gaggtgaaat tcgtagatat tcgc--aaga acaccagtgg tgcgagtgta gaggtgaaat tcgtagatat tcgc--aaga acaccagtgg tccgagtgta gaggtgaaat tcgtagatat tcgg--agga acaccagtgg

710 720 730 740

cgaagg--cg gctcactggc tcggtactga cgctg-aggt gcg-aaagcg cgaagc--ag gctcactggt ccggtactga cgctg-aggt gcg-aaagcg cgaagg--cg gctcactggc tcggtactga cgctg-aggt gcg-aaagcg cgaagg--cg gctcactggt ccggtactga cgctg-aggt gcg-aaagcg cgaagg--cg gctcactggc tcggtactga cgctg-aggt gcg-aaagcg cgaagg--cg gctcactggt ccggtactga cgctg-aggt gcg-aaagcg cgaagg--cg gctcactggt ccggtactga cgctg-aggt gcg-aaagcg cgaagg--cg gctcactggt ccggtactga cgctg-aggt gcg-aaagcg cgaagg--cg gctcactggt ccggtactga cgctg-aggt gcg-aaagcg cgaagg--cg gctcactggt ccggtactga cgctg-aggt gcg-aaagcg cgaagg--cg gctcactggt ccggtactga cgctg-aggt gcg-aaagcg cgaagg--cg gctcactggt ccggtactga cgctg-aggt gcg-aaagcg cgaagg--cg gctcactggc tcggtactga cgctg-aggt gcg-aaagcg cgaagg--cg gctcactggt ccggtactga cgctg-aggt gcg-aaagco cgaagg--cg gctcactggt ccggtactga cgctg-aggt gcg-aaagcg cgaagg--cg gctcactggc tcggtactga cgctg-aggt gcg-aaagcg cgaagg--cg gctcactggc tcggtactga cgctg-aggt gcg-aaagco cgaagg--cg gctcactggt ccggtactga cgctg-aggt gcg-aaagcg cgaagg--cg gctcactggc tcggtactga cgctg-aggt gcgaaaagcg cgaagg--cg gctcactggt ccggtactga cgctg-aggt gcg-aaagcg cgaagg--cg gctcactggt ctggtactga cgctg-aggt gcg-aaagcg cgaagg--cg gctcactggt ccggtactga cgctg-aggt gcg-aaagcg cgaagg--cg gctcactggt ccggaactga cgctg-aggt gcg-aaagcg cgaagg--cg gctcactggt ctggaactga cgctg-aggt gcg-aaagcg cgaagg--cg gctcactggt ctggaactga cgctg-aggt gcg-aaagcg cgaagg--cg gctcactggt ctggtactga cgctg-aggt gcg-aaagcg cgaagg--cg gctcactggt ctggtactga cgctgaaggt gcg-aaagcg cgaagg--cg gctcactggt ctggtactga cgctg-aggt gcg-aaagcg cgaagg--cg gctcactggt ctggtactga cgctg-aggt gcg-aaagcg cgaagg--cg gctcactggt ctggtactga cgctg-aggt gcg-aaagcg cgaagg--cg gctcactggt ctggtactga cgctg-aggt gcg-aaagcg cgaagg--cg gctcactggt ctggtactga cgctg-aggt gcg-aaagcg cgaagg--cg gctcactggt ctggtactga cgctg-aggt gcg-aaagcg cgaagg--cg gctcactggc ccgatactga cgctg-aggc acg-aaagcg cgaagg--cg gctcactggc ccgatactga cgctg-aggc acg-aaagcg cgaagg--cg gctcactggc ccgatactga cgctg-aggc acg-aaagcg cgaaaggcgg ctccactggc ccgatactga cgctg-aggc acg-aaagcg cgaagg--cg gctcactggc ccgatactga cgctg-aggc acg-aaagcg 
NK22 $L$. sericea SA WC21.1L L. pauciflora SA XHRla $L$. simsiana SA Betal106f $L$. pungens SA $R$. palustris (ATCC17001) $M$. nodulans (ORS2060)

Az. Caulinodans (LMG6465)

$R$. rubrum (LMGATCC11170)

A. magnetotacticum (M58171)

Az. brasilense (NCIMB11860)

Az. Iipoferum (NCIMB11861)

$R$. acidophila (M34128)

A. clevelandensis (M69186)

S. morelense (LMG21331) T

$M$. amorphae (LMG18977) T

M. ciceri (LMG14989) T

M. huakuii (LMG14107) T

M. loti (LMG6125) T

M. plurifarium (LMG11892) T

B. elkanii (LMG6134) T

B. beta (LMG21987) T

B. yuanmingense (CCBAU10071) T

S. medicae (A321) T

M. mediterraneum (LMG17148) T

$M$. septentrionale (SDW014)T

$M$. temperatum (SDW018) T

$M$. thiogangeticum (SJT) T $M . t i a n s h a n e n s e$ (A-1BS) T WC12.1a $L$. sepiaria SA

WC19.1b L. cytisoides SA WC19.1C $L$. cytisoides SA WC23.1b $L$. multiflora SA WC23.1C $L$. multiflora SA WC23.1d L. multiflora SA WC28.1F $L$. sericea SA WC28.1g $L$. sericea SA WC28.1h $L$. sericea SA WC28.1i $L$. sericea SA WC33b L. sericea SA WC33c $L$. sericea SA WC33e $L$. sericea SA WC33h $L$. sericea SA $S$. americanum (CFNEI156) T

S. arboris (LMG14919) T

S. fredii (LMG6217) T

S. kostiense (LMG19227)

S. kummerowiae (CCBAU71714) T

S. meliloti (LMG6133) T

BH1LSC $L$. spinescens SA BH2LSa $L$. spinescens SA BH3LSd $L$. spinescens SA BW1LSab $L$. spinescens SA BW2LSa $L$. spinescens SA BW2LSb $L$. spinescens SA BW2LSC $L$. spinescens SA BW3LSd $L$. spinescens SA $B$. canariense (MSFC2-M3-R-3) T B. japonicum (LMG6138) T B. liaoningense (LMG18230) T Betal106d L. pungens SA Betal106a $L$. pungens SA NK22 $L$ sericea SA WC21.1L L. pauciflora SA XHR1a $L$. simsiana SA Betal106f $L$. pungens SA $R$. palustris (ATCC17001) $M$. nodulans (ORS2060)

Az. caulinodans (LMG6465)

R. rubrum (LMGATCC11170)

A. magnetotacticum (M58171)

Az. brasilense (NCIMB11860)

Az. Iipoferum (NCIMB11861)

R. acidophila (M34128)

A. clevelandensis (M69186)

S. morelense (LMG21331) T

cgaagg--cg gctcactggc ccgatactga cgctg-aggc acg-aaagcg cgaagg--cg gctcactggc ccgatactga cgctg-aggc acg-aaagcg cgaagg--cg gctcactggc ccggtactga cgctg-aggc acg-aaagcg cgaagg--cg gctcactggc ccgatactga cgctg-aggc acg-aaagcg cgaagg--cg gctcactggg ccattactga cgctg-aggc acg-aaagcg cgaagg--cg gctgtctggt ccggttctga cgctg-aggc gcg-aaagcg cgaagg--cg gccaactggc tcgatactga cgctg-aggt gcg-aaagcg cgaagg--cg gccacctggc gcggtattga cgctg-aggc gcg-aaagcg cgaagg--cg actacctggc cgtsgattga ssctc-atgt gcg-aaagccgaagg--cg gccatctgga cggacactga cgctg-aggc gcg-aaagcg cgaagg--cg gccatctgga cggacactga cgctg-aggc gcg-aaagcg cgaagg--cg gctcactggc tcgatactga cgctg-aggt gc---aagc cgaagg--cg gctcactggc ccgatactga cgctg-aggc acg-aaagcg cgaagg--cg gctcactggt ccattactga cgctg-aggt gcg-aaagcg cgaagg--cg gctcactggt ccggtactga cgctg-aggt gcg-aaagcg cgaagg--cg gctcactggc tcggtactga cgctg-aggt gcg-aaagcg cgaagg--cg gctcactggt ccggtactga cgctg-aggt gcg-aaagco cgaagg--cg gctcactggc tcggtactga cgctg-aggt gcg-aaagcg cgaagg--cg gctcactggt ccggtactga cgctg-aggt gcg-aaagcg cgaagg--cg gctcactggc ccgatactga cgctg-aggc acg-aaagco cgaagg--cg gctcactggc ccgatactga cgctg-aggc acg-aaagcg cgaagg--cg gctcactggc ccggtactga cgctg-aggc acg-aaagcg cgaagg--cg gctcactggt ctggtactga cgctg-aggt gcg-aaagcg

760

$\operatorname{tgggg--ag-10}$ - caaacagg atta-gatac cetggtagte cacgecgt-tgggg--ag- --caaacagg atta-gatac cctggtagtc cacgccgt-tgggg--ag- --caaacagg atta-gatac cctggtagtc cacgccgt-tgggg--ag- --caaacagg atta-gatac cctggtagtc cacgccgt-tgggg--ag- --caaacagg atta-gatac cctggtagtc cacgccgt-tgggg--ag- --caaacagg atta-gatac cctggtagtc cacgccgt-tgggg--ag- --caaacagg atta-gatac cctggtagtc cacgccgt-tgggg--ag- --caaacagg atta-gatac cctggtagtc cacgccgt-tgggg--ag- --caaacagg atta-gatac cctggtagtc cacgccgt-tgggg--ag- --caaacagg atta-gatac cctggtagtc cacgccgt-tgggg--ag- --caaacagg atta-gatac cctggtagtc cacgccgt-tgggg--ag- --caaacagg atta-gatac cctggtagtc cacgccgt-tgggg--ag- --caaacagg atta-gatac cctggtagtc cacgccgt-tgggg--ag- --caaacagg atta-gatac cctggtagtc cacgccgt-tgggg--ag- --caaacagg atta-gatac cctggtagtc cacgccgt-tgggg--ag- --caaacagg atta-gatac cctggtagtc cacgccgt-tgggg--ag- --caaacagg atta-gatac cctggtagtc cacgccgt-tgggg--ag- --caaacagg atta-gatac cctggtagtc cacgccgt-tgggg--ag- --caaacagg atta-gatac cctggtagtc cacgccgt-tgggg--ag- --caaacagg atta-gatac cctggtagtc cacgccgt-tgggg--ag- --caaacagg atta-gatac cctggtagtc cacgccgt-tgggg--ag- --caaacagg atta-gatac cctggtagtc cacgccgt-tgggg--ag- --caaacagg atta-gatac cctggtagtc cacgccgt-tgggg--ag- --caaacagg atta-gatac cctggtagtc cacgccgt-tgggg--ag- --caaacagg atta-gatac cctggtagtc cacgccgt-tgggg--gaa gcaaaacagg attaggatac cctgggtagt cccacgcccg tgggg--ag- --caaacagg atta-gatac cctggtagtc cacgccgt-tgggg--ag- --caaacagg atta-gatac cctggtagtc cacgccgt-tgggg--ag- --caaacagg atta-gatac cctggtagtc cacgccgt-tgggg--ag- --caaacagg atta-gatac cctggtagtc cacgccgt-tgggg--ag- --caaacagg atta-gatac cctggtagtc cacgccgt-tgggg--ag- --caaacagg atta-gatac cctggtagtc cacgccgt-tgggg--ag- --caaacagg atta-gatac cctggtagtc cacgccgt-tgggg--ag- --caaacagg atta-gatac cctggtagtc cacgccgt-tgggg--ag- --caaacagg atta-gatac cctggtagtc cacgccgt-tgggggagg- --caaacagg atta-gatac cctggtagtc cacgccgt-tgggg--ag- --caaacagg atta-gatac cctggtagtc cacgccgt-tgggg--ag- --caaacagg atta-gatac cctggtagtc cacgccgt-tgggg--ag- --caaacagg atta-gatac cctggtagtc cacgccgt-tgggg--ag- --caaacagg atta-gatac cctggtagtc cacgccgt-tgggg--ag- --caaacagg atta-gatac cctggtagtc cacgccgt-tgggg--ag- --caaacagg atta-gatac cctggtagtc cacgccgt-tgggg--ag- --caaacagg atta-gatac cctggtagtc cacgctgt-tgggg--ag- --caaacagg atta-gatac cctggtagtc cacgccgt-tgggg--ag- --caaacagg atta-gatac cctggtagtc cacgccgt-tgggg--ag- --caaacagg atta-gatac cctggtagtc cacgccgt-tgggg--ag- --caaacagg atta-gatac cctggtagtc cacgccgt-tgggg--ag- --caaacagg atta-gatac cctggtagtc cacgccgt-tgggg--ag- --caaacagg atta-gatac cctggtagtc cacgccgt-tgggg--ag- --caaacagg atta-gatac cctggtagtc cacgccgt-tgggg--ag- --caaacagg atta-gatac cctggtagtc cacgccgt-- 
M. amorphae (LMG18977)T

M. ciceri (LMG14989)T

M. huakuii (LMG14107) T

M. loti (LMG6125) T

M. plurifarium (LMG11892) T

B. elkanii (LMG6134) T

B. beta (LMG21987) T

B. yuanmingense (CCBAU10071) T

S. medicae (A321) T

M. mediterraneum (LMG17148) T

M. septentrionale (SDW014) T

$M$. temperatum (SDW018) T

$M$. thiogangeticum (SJT) T M.tianshanense (A-1BS) T WC12.1a $L$. sepiaria SA

WC19.1b $L$. cytisoides SA WC19.1c L. cytisoides SA WC23.1b L. multiflora SA WC23.1c $L$. multiflora SA WC23.1d L. multiflora SA WC28.1F $L$, sericea SA WC28.1g $L$. sericea SA WC28.1h L. sericea SA WC28.1i L. sericea SA WC33b $L$. sericea SA WC33c $L$. sericea SA WC33e $L$. sericea SA WC33h L. sericea SA

$S$. americanum (CFNEI156) T

$S$. arboris (LMG14919) T

$S$. fredii (LMG6217) T

S. kostiense (LMG19227)

S. kummerowiae (CCBAU71714) T

S. meliloti (LMG6133) T

BH1LSC L. spinescens SA BH2LSa L. spinescens SA BH3LSd $L$. spinescens SA BW1LSab $L$. spinescens SA BW2LSa L. spinescens SA BW2LSb $L$. spinescens SA BW2LSC $L$. spinescens SA BW3LSd $L$. spinescens SA B. canariense (MSFC2-M3-R-3) T B. japonicum (LMG6138) T $B$. liaoningense (LMG18230) T Betall06d L. pungens SA Betal106a L. pungens SA NK22 $L$. sericea SA

WC21.1L $L$. pauciflora SA XHR1a L. simsiana SA

Betal106f L. pungens SA

$R$. palustris (ATCC17001)

$M$. nodulans (ORS2060)

Az. Caulinodans (LMG6465)

$R$. rubrum (LMGATCC11170)

A. magnetotacticum (M58171)

Az. brasilense (NCIMB11860)

Az. lipoferum (NCIMB11861)

$R$. acidophila (M34128)

A. Clevelandensis (M69186)

$S$. morelense (LMG21331) T

M. amorphae (LMG18977) T

M. Ciceri (LMG14989) T

M. huakuii (LMG14107)T

M. loti (LMG6125) T

M. plurifarium (LMG11892) T

B. elkanii (LMG6134) T

B. beta (LMG21987) T

B. yuanmingense (CCBAU10071) T

S. medicae (A321) T

M. mediterraneum (LMG17148) T

$M$. septentrionale (SDW014) T

$M$. temperatum (SDW018) T

tgggg--ag- --caaacagg atta-gatac cctggtagtc cacgccgt-tgggg--ag- --caaacagg atta-gatac cctggtagtc cacgccgt-tgggg--ag- --caaacagg atta-gatac cctggtagtc cacgccgt-tgggg--ag- --caaacagg atta-gatac cctggtagtc cacgccgt-tgggg--ag- --caaacagg atta-gatac cctggtagtc cacgccgt-tgggg--ag- --caaacagg atta-gatac cctggtagtc cacgctgt-tgggg--ag- --caaacagg atta-gatac cctggtagtc cacgccgt-tgggg--ag- --caaacagg atta-gatac cctggtagtc cacgccgt-tgggg--ag- --caaacagg atta-gatac cctggtagtc cacgccgt--

$$
810
$$

830

840

850

--aaacgatg ga-agctagc cgttggc-aa gttt-acttg tcggtggcgc --aaacgatg ga-agctagc cgttggc-aa gttt-acttg tcggtggcgc --aaacgatg ga-agctagc cgttggc-aa gttt-acttg tcggtggcgc --aaacgatg ga-agctagc cgttggc-aa gttt-acttg tcggtggcgc --aaacgatg ga-agctagc cgttggc-aa gttt-acttg tcggtggcgc --aaacgatg ga-agctagc cgttggc-aa gttt-acttg tcggtggcgc --aaacgatg ga-agctagc cgttggc-aa gttt-acttg tcggtggcgc --aaacgatg ga-agctagc cgttggc-aa gttt-acttg tcggtggcgc --aaacgatg ga-agctagc cgttggc-aa gttt-acttg tcggtggcgc --aaacgatg ga-agctagc cgttggc-aa gttt-acttg tcggtggcgc --aaacgatg ga-agctagc cgttggc-aa gttt-acttg tcggtggcgc --aaacgatg ga-agctagc cgttggc-aa gttt-acttg tcggtggcgc --aaactatg ag-agctagc cgtcggc-aa gttt-acttg tcggtggcgc --aaacgatg ga-agctagc cgttggc-aa gttt-acttg tcggtggcgc --aaacgatg ga-agctagc cgttggc-aa gttt-acttg tcggtggcgc --aaactatg ag-agctagc cgtcggc-aa gttt-acttg tcggtggcgc --aaactatg ag-agctagc cgtcggc-aa gttt-acttg tcggtggcgc --aaacgatg ga-agctagc cgttggc-aa gttt-acttg tcggtggcgc --aactatg ag-agctagc cgtcggc-aa gttt-acttg tcggtggcgc --aaacgatg aa-tgttagc cgtcggg-ca gttt-actgt tcggtggcgc --aaacgatg aa-tgttagc cgtcggg-ca gttt-actgt tcggtggcgc --aaacgatg aa-tgttagc cgtcggg-ca gttt-actgt tcggtggcgc --aaacgatg aa-tgttagc cgtcggg-ca gttt-actgt tcggtggcgc --aaacgatg aa-tgttagc cgtcggg-ca gttt-actgt tcggtggcgc -aaacgatg aa-tgttagc cgtcggg-ca gttt-actgt tcggtggcgc --aaacgatg aa-tgttagc cgtcggg-ca gttt-actgt tcggtggcgc taaaacgatg aa-tgttagc cgtcggg-ca gttt-actgt tcggtggcgc --aaacgatg aa-tgttagc cgtcggg-ca gttt-actgt tcggtggcgc --aaacgatg aa-tgttagc cgtcggg-ca gttt-actgt tcggtggcgc --aaacgatg aa-tgttagc cgtcggg-ca gttt-actgt tcggtggcgc --aaacgatg aa-tgttagc cgtcggg-ca gttt-actgt tcggtggcgc --aaacgatg aa-tgttagc cgtcggg-ca gttt-actgt tcggtggcgc --aaacgatg aa-tgttagc cgtcggg-ca gttt-actgt tcggtggcgc --aaacgatg aa-tgccagc cgttagt-gg gttt-actca ctagtggcgc --aaacgatg aa-tgccagc cgttagt-gg gttt-actca ctagtggcgc --aaacgatg aa-tgccagc cgttagt-gg gttt-actca ctagtggcgc --aaacgatg aa-tgccagc cgttagt-gg gttt-actca ctagtggcgc --aaacgatg aa-tgccagc cgttagt-gg gttt-actca ctagtggcgc --aaacgatg aa-tgccagc cgttagt-gg gttt-actca ctagtggcgc --aaacgatg aa-tgccagc cgttagt-gg gttt-actca ctagtggcgc --aaacgatg aa-tgccagc cgttagt-gg gttt-actca ctagtggcgc --aaacgatg aa-tgccagc cgttagt-gg gttt-actca ctagtggcgc --aaacgatg aa-tgccagc cgttagt-gg gttt-actca ctagtggcgc --aaacgatg aa-tgctagc cgttggg-gt gcat-gcacc tcagtggcgc --aaacgatg ga-tgctagc cgttggg-ga gctt-gctct tcagtggcgc --aaacgatg ag-tgctaga tgtcggg-gt acat-gtacc tcggtgtcgc --aaacgatg ag-tgctaag ttgttgg-gt gcat-gcacc tcagtgacgc --aaacgatg aa-tgctaga cgctggg-gt gcat-gcact tcggtgtcgc --aaacgatg aa-tgctaga cgtcggg-gt gcat-gcact tcggtgtcgc --aaacgatg ga-tgctagc cgttagg-ca gctt-gctgc ttagtggcgc --aaacgatg aa-tgccagc cgttgga-aa gttt-acttt tcagtggcgc --aaacgatg aa-tgttagc cgtcggg-ca gttt-actgt tcggtggcgc --aaacgatg ga-agctagc cgttggc-aa gttt-acttg tcggtggcgc --atactatg aagagctagc cgtcggctaa gttt-acttg tcggtggcgc --aaacgatg ga-agctagc cgttggc-aa gttt-acttg tcggtggcgc --aaacgatg ag-agctagc cgtcggc-aa gttt-acttg tcggtggcgc --aaacgatg ga-agctagc cgttggc-aa gttt-acttg tcggtggcgc --aaacgatg aa-tgccagc cgttagt-gg gttt-actca ctagtggcgc --aaacgatg aa-tgccagc cgttagt-gg gttt-actca ctagtggcgc --aaacgatg aa-tgccagc cgttagt-gg gttt-actca ctagtggcgc --aaacgatg aa-tgttagc cgtcggg-ca gttt-actgt tcggtggcgc

860 agctaacgca ttaagcttcc cgcct-gggg agtacggtcg caagattaaa 
M. thiogangeticum (SJT) T $M . t i a n s h a n e n s e$ (A-1BS) T WC12.1a $L$. sepiaria SA WC19.1b $L$. cytisoides SA WC19.1C $L$. cytisoides SA WC23.1b L. multiflora SA WC23.1c $L$. multiflora SA WC23.1d L. multiflora SA WC28.1F $L$. sericea SA WC28.1g $L$. sericea SA WC28.1h $L$. sericea SA WC28.1i $L$, sericea SA WC33b $L$, sericea SA WC33c $L$. sericea SA WC33e $L$. sericea SA WC33h $L$. sericea SA $S$. americanum (CFNEI156) T

$S$. arboris (LMG14919) T

S. fredii (LMG6217) T

S. kostiense (LMG19227)

S. kummerowiae (CCBAU71714) T S. meliloti (LMG6133) T BH1LSC L. spinescens SA BH2LSa $L$. spinescens SA BH3LSd $L$. spinescens SA BW1LSab $L$. spinescens SA BW2LSa $L$. spinescens SA BW2LSb $L$. spinescens SA BW2LSC $L$. spinescens SA BW3LSd $L$. spinescens SA $B$. canariense (MSFC2-M3-R-3) T B. japonicum (LMG6138) T B. liaoningense (LMG18230) T Betal106d L. pungens SA Betal106a L. pungens SA NK22 L. sericea SA WC21.1L $L$. pauciflora SA XHRla L. simsiana SA Betal106f $L$. pungens SA $R$. palustris (ATCC17001) $M$. nodulans (ORS2060) Az. Caulinodans (LMG6465)

$R$. rubrum (LMGATCC11170)

A. magnetotacticum (M58171)

Az. brasilense (NCIMB11860)

$A z$. Iipoferum (NCIMB11861)

$R$. acidophila (M34128)

A. Clevelandensis (M69186)

S. morelense (LMG21331) T

M. amorphae (LMG18977) T

M. ciceri (LMG14989) T

M. huakuii (LMG14107) T

M. loti (LMG6125) T

M. plurifarium (LMG11892) T

B. elkanii (LMG6134) T

B. beta (LMG21987) T

$B$. yuanmingense (CCBAU10071) T

S. medicae (A321) T

M. mediterraneum (LMG17148) T

$M$. septentrionale (SDW014)T

$M$. temperatum (SDW018) T

$M$. thiogangeticum (SJT) T $M$. tianshanense (A-1BS) T WC12.1a $L$. sepiaria SA

WC19.1b L. cytisoides SA WC19.1C $L$. cytisoides SA WC23.1b L. multiflora SA

WC23.1c L. multiflora SA

WC23.1d L. multiflora SA

WC28.1F $L$. sericea SA

WC28.1g $L$. sericea SA WC28.1h $L$. sericea SA WC28.1i $L$ sericea SA WC33b L. sericea SA WC33C L. sericea SA agctaacgca ttaagcttcc cgcct-gggg agtacggtco caagattaaa agctaacgca ttaagcttcc cgcct-gggg agtacggtcg caagattaaa agctaacgca ttaagcttcc cgcct-gggg agtacggtcg caagattaaa agctaacgca ttaagcttcc cgcctggggg agtacggtco caagattaaa agctaacgca ttaagcttcc cgcct-gggg agtacggtcg caagattaaa agctaacgca ttaagcttcc cgcct-gggg agtacggtcg caagattaaa agctaacgca ttaagcttcc cgcct-gggg agtacggtcg caagattaaa agctaacgca ttaagcttcc cgcct-gggg agtacggtcg caagattaaa agctaacgca ttaagcttcc cgcct-gggg agtacggtcg caagattaaa agctaacgca ttaagctctc cgcct-gggg agtacggtcg caagattaaa agctaacgca ttaagcttcc cgcct-gggg agtacggtcg caagattaaa agctaacgca ttaagcttcc cgcct-gggg agtacggtcg caagattaaa agctaacgca ttaagctctc cgcct-gggg agtacggtcg caagattaaa agctaacgca ttaagctctc cgcct-gggg agtacggtcg caagattaaa agctaacgca ttaagcttcc cgcct-gggg agtacggtco caagattaaa agctaacgca ttaagctctc cgcct-gggg agtacggtcg caagattaaa agctaacgca ttaaacattc cgcct-gggg agtacggtcg caagattaaa agctaacgca ttaaacattc cgcct-gggg agtacggtco caagattaaa agctaacgca ttaaacattc cgcct-gggg agtacggtcg caagattaaa agctaacgca ttaaacattc cgcct-gggg agtacggtcg caagattaaa agctaacgca ttaaacattc cgcct-gggg agtacggtcg caagattaaa agctaacgca ttaaacattc cgcct-gggg agtacggtcg caagattaaa agctaacgca ttaaacattc cgcct-gggg agtacggtcg caagattaaa agctaacgca ttaaacattc cgcct-gggg agtacggtcg caagattaaa agctaacgca ttaaacattc cgcct-gggg agtacggtcg caagattaaa agctaacgca ttaaacattc cgcct-gggg agtacggtco caagattaaa agctaacgca ttaaacattc cgcctggggg agtacggtcg caagattaaa agctaacgca ttaaacattc cgcct-gggg agtacggtcg caagattaaa agctaacgca ttaaacattc cgcct-gggg agtacggtcg caagattaaa agctaacgca ttaaacattc cgcct-gggg agtacggtcg caagattaaa agctaacgct ttaagcattc cgcct-gggg agtacggtco caagattaaa agctaacgct ttaagcattc cgcct-gggg agtacggtcg caagattaaa agctaacgct ttaagcattc cgcct-gggg agtacggtcg caagattaaa agctaacgct ttaagcattc cgcct-gggg agtacggtcg caagattaaa agctaacgct ttaagcattc cgcct-gggg agtacggtcg caagattaaa agctaacgct ttaagcattc cgcct-gggg agtacggtcg caagattaaa agctaacgct ttaagcattc cgcct-gggg agtacggtcg caagattaaa agctaacgct ttaagcattc cgcct-gggg agtacggtcg caagattaaa agctaacgct ttaagcattc cgcct-gggg agtacggtcg caagattaaa a--taacgct ttaagcattc cgcct-gggg agtacggtcg caagattaaa cgctaacgct ttaagcattc cgcct-gggg agtacggtcg caagattaaa agctaacgcc ttaagcatcc cgcct-gggg agtacggtcg caagattaaa agctaacgca ttaagcactc cgcct-gggg agtacggccg caaggttaaa acgtaacgcg ttaagcactc cgcct-gggg agtacggccg caaggttaaa -cctaacgca ttaagcattc cgcct-gggg agtacggccg caaggttaaa ccgtaacgca ttaagcattc cgcct-gggg agtacggccg caaggttaaa agctaacgct ttaagcatcc cgcct-gggg agtacggtcg caagattaaa agctaacgct ttaagcattc cgcct-gggg agtacggtcg caagattaaa agctaacgca ttaaacattc cgcct-gggg agtacggtcg caagattaaa agctaacgca ttaagcttcc cgcct-gggg agtacggtcg caagattaaa agctaacgca ttaagctctc cgcct-gggg agtacggtcg caagattaaa acgtaacgca ttaagcttgc cgcct-gggg agtacggtcg caagattaaa acgtaacgca ttaagctctc cgcct-gggg agtacggtcg caagattaaa agctaacgca ttaagcttcc cgcct-gggg agtacggtcg caagattaaa agctaacgct ttaagcattc cgcct-gggg agtacggtcg caagattaaa agctaacgct ttaagcattc cgcct-gggg agtacggtcg caagattaaa agctaacgct ttaagcattc cgcct-gggg agtacggtcg caagattaaa agctaacgca ttaaacattc cgcct-gggg agtacggtcg caagattaaa

910 920 930 940 950 actcaaagga a-ttgac-gg gggcccgcac -aagcggt-g gagcatgtgg actcaaagga a-ttgac-gg gggcccgcac -aagcggt-g gagcatgtgg actcaaagga a-ttgac-gg gggcccgcac -aagcggt-g gagcatgtgg actcaaagga a-ttgac-gg gggcccgcac -aagcggt-g gagcatgtgg actcaaagga a-ttgac-gg gggcccgcac -aagcggt-g gagcatgtgg actcaaagga a-ttgac-gg gggcccgcac -aagcggt-g gagcatgtgg actcaaagga a-ttgac-gg gggcccgcac -aagcggt-g gagcatgtgg actcaaagga a-ttgacggg gggcccgcac -aagcggt-g gagcatgtgg actcaaagga a-ttgacggg gggcccgcac -aagcggt-g gagcatgtgg actcaaagga a-ttgac-gg gggcccgcac -aagcggt-g gagcatgtgg actcaaagga a-ttgac-gg gggcccgcac -aagcggt-g gagcatgtgg actcaaagga a-ttgac-gg gggcccgcac -aagcggt-g gagcatgtgg actcaaagga a-ttgac-gg gggcccgcac -aagcggt-g gagcatgtgg actcaaagga a-ttgac-gg gggcccgcac -aagcggt-g gagcatgtgg actcaaagga a-ttgac-gg gggcccgcac -aagcggtgg gagcatgtgg actcaaagga a-ttgac-gg gggcccgcac -aagcggt-g gagcatgtgg actcaaagga atttgac-gg gggcccgcac -aagcggt-g gagcatgtgg 
WC33e I, sericea SA

WC33h L. sericea SA

S. americanum (CFNEI156) T

$S$. arboris (LMG14919) T

S. fredii (LMG6217) T

S. kostiense (LMG19227)

S. kummerowiae (CCBAU71714) T

S. meliloti (LMG6133) T

BH1LSC $L$. spinescens SA

BH2LSa $L$. spinescens SA

BH3LSd $L$. spinescens SA

BW1LSab $L$. spinescens SA

BW2LSa $L$. spinescens SA

BW2LSb $L$. spinescens SA

BW2LSC $L$. spinescens SA

BW3LSd $L$. spinescens SA

$B$. canariense (MSFC2-M3-R-3) T

B. japonicum (LMG6138) T

$B$. liaoningense (LMG18230) T

Betallo6d L. pungens SA

Betal106a L. pungens SA

NK22 $L$. sericea SA

WC21.1L $L$. pauciflora SA

XHRla $L$. simsiana SA

Betal106f L. pungens SA

$R$. palustris (ATCC17001)

$M$. nodulans (ORS2060)

Az. caulinodans (LMG6465)

R. rubrum (LMGATCC11170)

A. magnetotacticum (M58171)

$A z$. brasilense (NCIMB11860)

$A z$. lipoferum (NCIMB11861)

$R$. acidophila (M34128)

A. clevelandensis (M69186)

S. morelense (LMG21331) T

$M$. amorphae (LMG18977) T

M. ciceri (LMG14989) T

M. huakuii (LMG14107) T

M. Loti (LMG6125) T

M. plurifarium (LMG11892) T

B. elkanii (LMG6134) T

B. beta (LMG21987) T

B. yuanmingense (CCBAU10071) T

S. medicae (A321) T

M. mediterraneum (LMG17148) T

$M$. septentrionale (SDW014)T

$M$. temperatum (SDW018) T

$M$. thiogangeticum (SJT) T

$M$. tianshanense (A-1BS) T

WC12.1a $L$. sepiaria SA

WC19.1b $L$. cytisoides SA

WC19.1c $L$. cytisoides SA

WC23.1b L. multiflora SA

WC23.1C $L$. multiflora SA

WC23.1d L. multiflora SA

WC28.1F $L$. sericea SA

WC28.1g $L$. sericea SA

WC28.1h $L$. sericea SA

WC28.1i $L$. sericea SA

WC33b L. sericea SA

WC33C L. sericea SA

WC33e L. sericea SA

WC33h L. sericea SA

S. americanum (CFNEI156) T

$S$. arboris (LMG14919) T

S. fredii (LMG6217) T

S. kostiense (LMG19227)

S. kummerowiae (CCBAU71714) T

S. meliloti (LMG6133) T

BH1LSC L. spinescens SA

BH2LSa $L$. spinescens SA

BH3LSd $L$. spinescens SA

BW1LSab $L$. spinescens SA

BW2LSa $L$. spinescens SA

BW2LSb $L$. spinescens SA actcaaagga a-ttgac-gg gggcccgcac -aagcggt-g gagcatgtgg actcaaagga a-ttgac-gg gggcccgcac -aagcggt-g gagcatgtgg actcaaagga a-ttgac-gg gggcccgcac -aagcggt-g gagcatgtgg actcaaagga a-ttgac-gg gggcccgcac -aagcggt-g gagcatgtgg actcaaagga a-ttgac-gg gggcccgcac -aagcggt-g gagcatgtgg actcaaagga a-ttgac-gg gggcccgcac -aagcggt-g gagcatgtgg actcaaagga a-ttgac-gg gggcccgcac -aagcggt-g gagcatgtgg actcaaagga a-ttgac-gg gggcccgcac -aagcggt-g gagcatgtgg actcaaagga a-ttgac-gg gggcccgcac -aagcggt-g gagcatgtgg actcaaagga a-ttgac-gg gggcccgcac -aagcggt-g gagcatgtgg actcaaagga a-ttgacggg gggcccgcac -aagcggt-g gagcatgtgg actcaaagga a-ttgac-gg gggcccgcac -aagcggt-g gagcatgtgg actcaaagga a-ttgac-gg gggcccgcac -aagcggt-g gagcatgtgg actcaaagga a-ttgac-gg gggcccgcac -aagcggt-g gagcatgtgg actcaaagga a-ttgac-gg gggcccgcac -aagcggt-g gagcatgtgg actcaaagga a-ttgac-gg gggcccgcac -aagcggt-g gagcatgtgg actcaaagga a-ttgac-gg gggcccgcac aaagcggt-g gagcatgtgg actcaaagga a-ttgac-gg gggcccgcac -aagcggt-g gagcatgtgg actcaaagga a-ttgac-gg gggcccgcac -aagcggt-g gagcatgtgg actcaaagga a-ttgac-gg gggcccgcac -aagcggt-g gagcatgtgg actcaaagga a-ttgac-gg gggcccgcac -aagcggt-g gagcatgtgg actcaaagga a-ttgac-gg gggcccgcac -aagcggt-g gagcatgtgg actcaaagga a-ttgac-gg gggcccgcac -aagcggt-g gagcatgtgg actcaaagga a-ttgac-gg gggcccgcac -aagcggt-g gagcatgtgg actcaaagga a-ttgac-gg gggcccgcac -aagcggt-g gagcatgtgg actcaaagga a-tt----gg ggg-ccgcac -aagcggt-g gagcatgtgg actcaaagga a-ttgac-gg gggcccgcac -aagcggt-g gagcatgtgg actcaaagga a-ttgac-gg ggg-ccgcac -aagcggt-g gagcatgtgo actcaaagga a-ttgac-gg gggcccgcac -aagcggt-g gagcatgtgg actcaaagga a-ttgac-gg gggcccgcac -aagcggt-g gagcatgtgg actcaaagga a-ttgac-gg gggcccgcac -aagcggt-g gagcatgtgg actcaaagga a-ttgac-gg gggcccgcac -aagcggt-g gagcatgtgg actcaaagga a-ttgac-gg gggc-cgcac -aagcggt-g gagcatgtgg actcaaagga a-ttgac-gg ggg-ccgcac -aagcggt-g gagcatgtgg acttaaagga a-ttgac-gg gggcccgcac -aagcggt-g gagcatgtgg actcaaagga a-ttgac-gg gggcccgcac -aagcggt-g gagcatgtgg actcaaagga a-ttgac-gg gggcccgcac -aagcggt-g gagcatgtgg actcaaagga a-ttgac-gg gggcccgcac -aagcggt-g gagcatgtgg actcaaagga a-ttgac-gg gggcccgcac -aagcggt-g gagcatgtgg actcaaagga a-ttgac-gg gggcccgcac -aagcggt-g gagcatgtgg actcaaagga a-ttgac-gg gggcccgcac -aagcggt-g gagcatgtgg actcaaagga a-ttgac-gg gggcccgcac -aagcggt-g gagcatgtgg actcaaagga a-ttgac-gg gggcccgcac -aagcggt-g gagcatgtgg actcaaagga a-ttgac-gg gggcccgcac -aagcggt-g gagcatgtgg
960
970
980
990
1000

ttaa-ttcg aagcaacgcg cagaacc-tt accagccett gacat-cccg tttaa-ttcg aagcaacgcg cagaacc-tt accagccett gacat-cccg tttaa-ttcg aagcaacgcg cagaacc-tt accagccett gacat-cccg tttaa-ttcg aagcaacgcg cagaacc-tt accagccett gacat-cccg tttaa-ttcg aagcaacgcg cagaacc-tt accagccctt gacat-cccg tttaa-ttcg aagcaacgcg cagaacc-tt accagccett gacat-cccg tttaa-ttcg aagcaacgcg cagaaccttt accagccett gacat-cccg tttaa-ttcg aagcaacgcg cagaacc-tt accagccctt gacat-cccg tttaa-ttcg aagcaacgcg cagaacc-tt accagccctt gacat-cccg tttaa-ttcg aagcaacgcg cagaacc-tt accagccett gacat-cccg tttaa-ttcg aagcaacgcg cagaacc-tt accagccett gacat-cccg tttaatttcg aagcaacgcg cagaacc-tt accagccctt gacat-cccg tttaa-ttcg aagcaacgcg cagaacc-tt accagccctt gacat-cccg tttaa-ttcg aagcaacgcg cagaacc-tt accagccctt gacat-cccg tttaa-ttcg aagcaacgcg cagaacc-tt accagccett gacat-cccg tttaa-ttcg aagcaacgcg cagaacc-tt accagccett gacat-cccg tttaa-ttcg aagcaacgcg cagaacc-tt accagccett gacat-cccg tttaa-ttcg aagcaacgcg cagaacc-tt accagccett gacat-cccg tttaa-ttcg aagcaacgcg cagaacc-tt accagccett gacat-cccg tttaa-ttcg aagcaacgcg cagaacc-tt accagccctt gacat-cccg tttaa-ttcg aagcaacgcg cagaacc-tt accagccett gacat-cccg tttaa-ttcg aagcaacgcg cagaacc-tt accagccett gacat-cccg tttaa-ttcg aagcaacgcg cagaacc-tt accagccett gacat-cccg tttaa-ttcg aagcaacgcg cagaacc-tt accagccett gacatccccg tttaa-ttcg aagcaacgcg cagaacc-tt accagccett gacat-cccg tttaa-ttcg aagcaacgcg cagaacc-tt accagccett gacat-cccg tttaa-ttcg aagcaacgcg cagaacc-tt accagccctt gacat-cccg tttaa-ttcg aagcaacgcg cagaacc-tt accagccett gacat-cccg tttaa-ttcg aagcaacgcg cagaacc-tt accagccett gacat-cccg tttaa-ttcg aagcaacgcg cagaacc-tt accagccett gacat-cccg tttaa-ttcg aagcaacgcg cagaacc-tt accagccctt gacat-cccg 
BW2LSC $L$. spinescens SA BW3LSd $L$. spinescens SA $B$. canariense (MSFC2-M3-R-3) T B. japonicum (LMG6138) T B. liaoningense (LMG18230) T Betal106d L. pungens SA Betall06a L. punges SA NK22 $L$. sericea SA WC21.1L $L$. pauciflora SA XHR1a $L$. simsiana SA Betal106f L. pungens SA $R$. palustris (ATCC17001) $M$. nodulans (ORS2060) Az. Caulinodans (LMG6465)

R. rubrum (LMGATCC11170)

A. magnetotacticum (M58171)

Az. brasilense (NCIMB11860)

Az. lipoferum (NCIMB11861)

R. acidophila (M34128)

A. clevelandensis (M69186)

$S$. morelense (LMG21331) T

$M$. amorphae (LMG18977) T

M. ciceri (LMG14989) T

M. huakuii (LMG14107) T

M. loti (LMG6125) T

M. plurifarium (LMG11892) T

B. elkanii (LMG6134) T

B. beta (LMG21987) T

B. yuanmingense (CCBAU10071) T

S. medicae (A321) T

M. mediterraneum (LMG17148) T

$M$. septentrionale (SDW014) T

$M$. temperatum (SDW018) T

$M$. thiogangeticum (SJT) T

M.tianshanense (A-1BS) T

WC12.1a L. sepiaria SA

WC19.1b L. cytisoides SA

WC19.1c $L$. cytisoides SA

WC23.1b L. multiflora SA

WC23.1c I multiflora SA

WC23.1d L. multiflora SA

WC28.1F $L$. sericea SA

WC28.1g $L$. sericea SA

WC28.1h $L$. sericea SA

WC28.1i L. sericea SA

WC33b $L$. sericea SA

WC33c L. sericea SA

WC33e L. sericea SA

WC33h $L$. sericea SA

S. americanum (CFNEI156) T

$S$. arboris (LMG14919) T

S. fredii (LMG6217) T

S. kostiense (LMG19227)

S. kummerowiae (CCBAU71714) T

S. meliloti (LMG6133) T

BH1LSC $L$. spinescens SA

BH2LSa $L$. spinescens SA

BH3LSd $L$. spinescens SA

BW1LSab L. spinescens SA

BW2LSa $L$. spinescens SA

BW2LSb $L$. spinescens SA

BW2LSc $L$. spinescens SA

BW3LSd $L$. spinescens SA

$B$. canariense (MSFC2-M3-R-3) T

B. japonicum (LMG6138) T

B. liaoningense (LMG18230) T

Betal106d L. pungens SA

Betal106a $L$. pungens SA NK22 $L$. sericea SA

WC21.1L $L$. pauciflora SA

XHRla $L$. simsiana SA

Betal106f L. pungens SA

$R$. palustris (ATCC17001)

$M$. nodulans (ORS2060)

Az. caulinodans (LMG6465) tttaa-ttcg aagcaacgcg cagaacc-tt accagccett gacat-cccg tttaa-ttcg aagcaacgcg cagaacc-tt accagccctt gacat-cccg tttaa-ttcg acgcaacgcg cagaacc-tt accagccett gacat-gtcc tttaa-ttcg acgcaacgcg cagaacc-tt accagccett gacat-gtcc tttaa-ttcg acgcaacgcg cagaacc-tt accagccett gacat-gtcc tttaa-ttcg acgcaacgcg cagaacc-tt accagccett gacat-cccg tttaa-ttcg acgcaacgcg cagaacc-tt accagccett gacat-ccco tttaa-ttcg acgcaacgcg cagaacc-tt accagccett gacat-gtcc tttaa-ttcg acgcaacgcg cagaacc-tt accagccett gacat-cccg tttaa-ttcg acgcaacgcg cagaacc-tt accagccctt gacat-gtcc tttaa-ttcg acgcaacgcg cagaacc-tt accagccett gacat-cccg tttaa-tt-- ------cgcg cagaacc-tt accagccctt gacat-gtcc tttaa-ttcg aagcaacgcg cagaacc-tt accatccctt gacat-ggca tttaa-ttcg aagcaacgcg cagaacc-tt accagcctt gacat-ggca tttaa-ttcg aagcaacgcg cagaacc-tt accagccett gacat-cccg tttaa-ttcg aagcaacgcg cagaacc-tt accagccctt gacat-ggga tttaa-ttcg aagcaacgcg cagaacc-tt accaaccctt gacat-gtcc tttaa-ttcg aagcaacgcg cagaacc-tt accaaccctt gacat-gtcc tttaa-ttcg aagcaacgcg cagaacc-tt accagcttt gacat-gtct tttaa-ttcg aagcaacgcg cagaacc-tt accagccett gacat-gtcc tttaa-ttcg aagcaacgcg cagaacc-tt accagccett gacat-cccg tttaa-ttcg aagcaacgcg cagaacc-tt accagccctt gacat-cccg tttaa-ttcg aagcaacgcg cagaacc-tt accagccctt gacat-cccg tttaa-ttcg aagcaacgcg cagaacc-tt acctgccett gacat-cccg tttaa-ttcg aagcaacgcg cagaacc-tt accagccett gacat-cccg tttaa-ttcg aagcaacgcg cagaacc-tt accagccett gacat-cccg tttaa-ttcg acgcaacgcg cagaacc-tt accagccett gacat-cccg tttaa-ttcg acgcaacgcg cagaacc-tt accagccctt gacat-gtcc tttaa-ttcg acgcaacgcg cagaacc-tt accagccett gacat-gtcc tttaa-ttcg aagcaacgcg cagaacc-tt accagccett gacat-cccg$$
1010
$$

1020

1030

1040

1050 gtcgc-ggtc tccagagatg gagacc-ttc agttcggctg gaccggtg-a gtcgc-ggtt tccagagatg gattcc-ttc agttcggctg gaccggtg-a gtcgc-ggtc tccagagatg gagacc-ttc agttcggctg gaccggtg-a gtcgc-ggtt accagagatg gtatcc-ttc agttcggctg gaccggtg-a gtcgc-ggtt tccagagatg gattcc-ttc agttcggctg gaccggtg-a gtcgc-ggtt tccagagatg gatacc-ttc agttcggctg gaccggtg-a gtcgc-ggtt tccagagatg gatacc-ttc agttcggctg gaccggtg-a gtcgc-ggtt tccagagatg gatacc-ttc agttcggctg gaccggtg-a gtcgc-ggtt tccagagatg gatacc-ttc agttcggctg gaccggtg-a gtcgc-ggtt tccagagatg gatacc-ttc agttcggctg gaccggtg-a gtcgc-ggtt tccagagatg gatacc-ttc agttcggctg gaccggtg-a gtcgc-ggtt tccagagatg gatacc-ttc agttcggctg gaccggtg-a gtcgcgggtt tccagagatg gatacc-ttc agttcggctg gaccggtg-a gtcgc-ggtt tccagagatg gatacc-ttc agttcggctg gaccggtg-a gtcgc-ggtt tccagagatg gatacc-ttc agttcggctg gaccggtg-a gtcgc-ggtt tccagagatg gatacc-ttc agttcggctg gaccggtg-a gtcgc-ggtt tccagagatg gatacc-ttc agttcggctg gaccggtg-a gtcgc-ggtt tccagagatg gatacc-ttc agttcggctg gaccggtg-a gtcgc-ggtt tccagagatg gatacc-ttc agttcggctg gaccggtg-a atcgc-ggat acgagagatc gtatcc-ttc agttcggctg gatcggag-a gtcgc-ggat acgagagatc gtatcc-ttc agttcggctg gaccggag-a atcgc-ggat acgagagatc gtatcc-ttc agttcggctg gatcggag-a gtcgc-ggaa acgagagatc gtttcc-ttc agttcggctg gaccggag-a atcgc-ggat acgagagatc g-tatc-tcc agttcggctg gatcggag-a atcgc-ggat acgagagatc gtatcc-ttc agttcggctg gatcggag-a atcgc-ggat tacagagatg ttttcc-ttc agttcggctg gatcggag-a atcgc-ggat tacagagatg ttttcc-ttc agttcggctg gatcggag-a atcgc-ggat tacagagatg ttttcc-ttc agttcggctg gatcggag-a atcgc-ggat tacagagatg ttttcc-ttc agttcggctg gatcggag-a atcgc-ggat tacagagatg ttttcc-ttc agttcggctg gatcggag-a atcgc-ggat tacagagatg ttttcc-ttc agttcggctg gatcggag-a atcgc-ggat tacagagatg ttttcc-ttc agttcggctg gatcggag-a atcgc-ggat tacagagatg ttttcc-ttc agttcggctg gatcggag-a aggac-cggt cgcagagatg tgacct-tct --cttcggag cctggagc-a aggac-cggt cgcagagatg tgacct-tct --cttcggag cctggagc-a aggac-cggt cgcagagatg tgaccc-tct --cttcggag cctggaac-a gtcgc-ggac tccagagatg gagttc-ttc agttcggctg gaccggag-a gtcgc-ggac tccagagacg gagttc-ttc agttcggctg gaccggagaa aggac-cggt cgcagagatg tgacct-tct --cttcggag cctggagc-a gtcgc-ggac tccagagacg gagttc-ttc agttcggctg gaccggag-a aggac-cggt cgcagagatg tgacct-tct --cttcggag cctggaac-a gtcgc-ggac tccagagatg gagttc-ttc agttcggctg gaccggag-a agga--acgt cgcagagacg cgacct-tct --cttcggag cctggagc-a ggc---tacg tggagagatt cacggt-tcc --cttcgggg acctgcac-a g-gac-gact tccggagacg gatttc-ttc ca-gcaatgg acctgcac-a 
R. rubrum (LMGATCC11170)

A. magnetotacticum (M58171)

Az. brasilense (NCIMB11860)

Az. lipoferum (NCIMB11861)

R. acidophila (M34128)

A. clevelandensis (M69186)

$S$. morelense (LMG21331) T

M. amorphae (LMG18977) T

$M$. ciceri (LMG14989) T

$M$. huakuii (LMG14107) T

M. loti (LMG6125) T

$M$. plurifarium (LMG11892) T

B. elkanii (LMG6134)T

B. beta (LMG21987) T

B. yuanmingense (CCBAU10071) T

S. medicae (A321) T

M. mediterraneum (LMG17148) T

$M$. septentrionale (SDW014) T

$M$. temperatum (SDW018) T

$M$. thiogangeticum (SJT) T

M.tianshanense (A-1BS) T

WC12.1a L. sepiaria SA

WC19.1b L. cytisoides SA

WC19.1c $L$. cytisoides SA

WC23.1b L. multiflora SA

WC23.1c $L$. multiflora SA

WC23.1d L. multiflora SA

WC28.1F L. sericea SA

WC28.1g $L$. sericea SA

WC28.1h $L$. sericea SA

WC28.1i L. sericea SA

WC33b $L$. sericea SA

WC33c $L$. sericea SA

WC33e L. sericea SA

WC33h $L$. sericea SA

S. americanum (CFNEI156) T

$S$. arboris (LMG14919) T

S. fredii (LMG6217) T

s. kostiense (LMG19227)

S. kummerowiae (CCBAU71714) T

S. meliloti (LMG6133) T

BH1LSC $L$. spinescens SA

BH2LSa $L$. spinescens SA

BH3LSd $L$. spinescens SA

BW1LSab $L$. spinescens SA

BW2LSa $L$. spinescens SA

BW2LSb $L$. spinescens SA

BW2LSC $L$. spinescens SA

BW3LSd $L$. spinescens SA

$B$. canariense (MSFC2-M3-R-3) T

B. japonicum (LMG6138) T

B. liaoningense (LMG18230) T

Betallo6d L. pungens SA

Betal106a $L$. punges SA

NK22 $L$. sericea SA

WC21.1L $L$. pauciflora SA

XHR1a L. simsiana SA

Betal106f L. pungens SA

$R$. palustris (ATCC17001)

$M$. nodulans (ORS2060)

Az. caulinodans (LMG6465)

$R$. rubrum (LMGATCC11170)

A. magnetotacticum (M58171)

$A z$. brasilense (NCIMB11860)

$A z$. lipoferum (NCIMB11861)

R. acidophila (M34128)

A. clevelandensis (M69186)

$S$. morelense (LMG21331) T

$M$. amorphae (LMG18977) T

M. ciceri (LMG14989) T

M. huakuii (LMG14107)T

M. loti (LMG6125)T

M. plurifarium (LMG11892) T

B. elkanii (LMG6134)T

B. beta (LMG21987) T tga---cact tccagagatg gaaggt-tcc --cttcgggg acacggtg-a cgtmt-gttt gccagagatg gtgact-tgt --cttcggga cgcgtcac-a actac-cggc tcgagagatc gggct--ttc agttcggctg ggtggaac-a actatggtcg ctcagagatg gcgtccttca ggttcggctg ggtggaag-a tggac-ggat agcagagatg ttatct-tct --cttcggag ccgagaac-a aggac-cggt cgcagagatg tgacct-tct --cttcggag cctggagc-a atcgc-ggat tacagagatg tattcc-ttc agttcggctg gatcggag-a gtcgc-ggtt tccagagatg gattcc-ttc agttcggctg gaccggtg-a gtcgc-ggtt tccagagatg gatacc-ttc agttcggctg gaccggtg-a gtcgc-ggtt tccagagatg gattcctttc agttcggctg gaccggtg-a gtcgc-ggtt tccagagatg gatacc-ttc agttcggctg gaccggtg-a gtcgc-ggtt accagaaatg gtttcc-ttc agttcggctg gaccggtg-a gtcgcgggac tccagagacg gagttc-ttc agttcggctg gaccggag-a aggac-cggt cgcagagatg tgacct-tct --cttcggag cctggaac-a aggac-cggt cgcagagatg tgaccc-tct --cttcggag cctggagc-a atcgc-ggat aggagagatc ctatcc-ttc agttcggctg gatcggag-a

$$
1060 \quad 1070 \quad 1080 \quad 1090
$$

1100 caggtgctgc atggctgtcg tcagctcgtg tcgtgagatg ttgggttaag caggtgctgc atggctgtcg tcagctcgtg tcgtgagatg ttgggttaag caggtgctgc atggctgtcg tcagctcgtg tcgtgagatg ttgggttaag caggtgctgc atggctgtcg tcagctcgtg tcgtgagatg ttgggttaag caggtgctgc atggctgtcg tcagctcgtg tcgtgagatg ttgggttaag caggtgctgc atggctgtcg tcagctcgtg tcgtgagatg ttgggttaag caggtgctgc atggctgtcg tcagctcgtg tcgtgagatg ttgggttaag caggtgctgc atggctgtcg tcagctcgtg tcgtgagatg ttgggttaag caggtgctgc atggctgtcg tcagctcgtg tcgtgagatg ttgggttaag caggtgctgc atggctgtcg tcagctcgtg tcgtgagatg ttgggttaag caggtgctgc atggctgtco tcagctcgtg tcgtgagatg ttgggttaag caggtgctgc atggctgtcg tcagctcgtg tcgtgagatg ttgggttaag caggtgctgc atggetgtcg tcagctcgtg tcgtgagatg ttgggttaag caggtgctgc atggctgtcg tcagctcgtg tcgtgagatg ttgggttaag caggtgctgc atggctgtcg tcagctcgtg tcgtgagatg ttgggttaag caggtgctgc atggctgtcg tcagctcgtg tcgtgagatg ttgggttaag caggtgctgc atggctgtcg tcagctcgtg tcgtgagatg ttgggttaag caggtgctgc atggctgtcg tcagctcgtg tcgtgagatg ttgggttaag caggtgctgc atggctgtcg tcagctcgtg tcgtgagatg ttgggttaag caggtgctgc atggctgtcg tcagctcgtg tcgtgagatg ttgggttaag caggtgctgc atggctgtcg tcagctcgtg tcgtgagatg ttgggttaag caggtgctgc atggctgtcg tcagctcgtg tcgtgagatg ttgggttaag caggtgctgc atggctgtcg tcagctcgtg tcgtgagatg ttgggttaag caggtgctgc atggetgtcg tcagctcgtg tcgtgagatg ttgggttgag caggtgctgc atggctgtcg tcagctcgtg tcgtgagatg ttgggttaag caggtgctgc atggctgtcg tcagctcgtg tcgtgagatg ttgggttaag caggtgctgc atggetgtcg tcagctcgtg tcgtgagatg ttgggttaag caggtgctgc atggctgtcg tcagctcgtg tcgtgagatg ttgggttaag caggtgctgc atggctgtcg tcagctcgtg tcgtgagatg ttgggttaag caggtgctgc atggetgtcg tcagctcgtg tcgtgagatg ttgggttaad caggtgctgc atggctgtcg tcagctcgtg tcgtgagatg ttgggttaag caggtgctgc atggctgtcg tcagctcgtg tcgtgagatg ttgggttaag caggtgctgc atggctgtcg tcagctcgtg tcgtgagatg ttgggttaag caggtgctgc atggctgtcg tcagctcgtg tcgtgagatg ttgggttaag caggtgctgc atggctgtcg tcagctcgtg tcgtgagatg ttgggttaag caggtgctgc atggctgtcg tcagctcgtg tcgtgagatg ttgggttaag caggtgctgc atggctgtcg tcagctcgtg tcgtgagatg ttgggttaag caggtgctgc atggctgtcg tcagctcgtg tcgtgagatg ttgggttaag caggtgctgc atggctgtcg tcagctcgtg tcgtgagatg ttgggttaag caggtgctgc atggctgtcg tcagctcgtg tcgtgagatg ttgggttaag caggtgctgc atggctgtcg tcagctcgtg tcgtgagatg ttgggttaag caggtgctgc atggctgtcg tcagctcgtg tcgtgagatg ttgggttaag caggtgctgc atggctgtcg tcagctcgtg tcgtgagatg ttgggttaag caggtgctgc atggctgtcg tcagctcgtg tcgtgagatg ttgggttaag caggtgctgc atggctgtcg tcagctcgtg tcgtgagatg ttgggttaag caggtgctgc atggctgtcg tcagctcgtg tcgtgagatg ttgggttaag caggtgctgc atggctgtcg tcagctcgtg tcgtgagatg ttgggttaag caggtgctgc atggctgtcg tcagctcgtg tcgtgagatg ttgggttaag gaggtgctgc atggetgtcg tcagctcgtg tcgtgagatg ttgggttaag caggtgctgc atggctgtcg tc-gctcgtg tcgtgagatg ttgggttaag caggtgctgc atggctgtcg tcagctcgtg tcgtgagatg ttgggttaag caggtgctgc atggctgtcg tcagctcotg tcgtgagatg ttgggttaag caggtgctgc atggctgtcg tcagctcgtg tcgtgagatg ttgggttaag caggtgctgc atggctgtcg tcagctcgtg tcgtgagatg ttgggttaag caggtgctgc atggctgtcg tcagctcgtg tcgtgagatg ttgggttaag caggtgctgc atggctgtcg tcagctcgtg tcgtgagatg ttgggttaag caggtgctgc atggctgtcg tcagctcgtg tcgtgagatg ttgggttaag caggtgctgc atggctgtcg tcagctcgtg tcgtgagatg ttgggttaag caggtgctgc atggctgtcg tcagctcgtg tcgtgagatg ttgggttaag 
B. yuanmingense (CCBAU10071) T S. medicae (A321) T

M. mediterraneum (LMG17148) T

$M$. septentrionale (SDW014) T

$M$. temperatum (SDW018) T

$M$. thiogangeticum (SJT) T

M.tianshanense (A-1BS) T

WC12.1a $L$. sepiaria SA

WC19.1b $L$. cytisoides SA

WC19.1C $L$. cytisoides SA

WC23.1b L. multiflora SA

WC23.1c L. multiflora SA

WC23.1d $L$. multiflora SA

WC28.1F $L$. sericea SA

WC28.1g $L$. sericea SA

WC28.1h $L$. sericea SA

WC28.1i $L$. sericea SA

WC33b $L$. sericea SA

WC33c $L$. sericea SA

WC33e $L$. sericea SA

WC33h I sericea SA

S. americanum (CFNEI156) T

$S$. arboris (LMG14919) T

S. fredii (LMG6217) T

S. kostiense (LMG19227)

S. kummerowiae (CCBAU71714) T

S. meliloti (LMG6133) T

BH1LSC $L$. spinescens SA

BH2LSa $L$. spinescens SA

BH3LSd $L$. spinescens SA

BW1LSab $L$. spinescens SA

BW2LSa $L$. spinescens SA

BW2LSb $L$. spinescens SA

BW2LSC $L$. spinescens SA

BW3LSd $L$. spinescens SA

$B$. canariense (MSFC2-M3-R-3) T

B. japonicum (LMG6138) T

B. liaoningense (LMG18230) T

Betallo6d L. pungens SA

Betal106a $L$. pungens SA

NK22 $L$. sericea SA

WC21.1L L. pauciflora SA

XHRla $L$. simsiana SA

Betal106f $L$. pungens SA

R. palustris (ATCC17001)

$M$. nodulans (ORS2060)

Az. Caulinodans (LMG6465)

$R$. rubrum (LMGATCC11170)

A. magnetotacticum (M58171)

Az. brasilense (NCIMB11860)

$A z$. lipoferum (NCIMB11861)

$R$. acidophila (M34128)

A. clevelandensis (M69186)

S. morelense (LMG21331) T

$M$. amorphae (LMG18977) T

M. ciceri (LMG14989) T

M. huakuii (LMG14107) T

M. Loti (LMG6125) T

M. plurifarium (LMG11892) T

B. elkanii (LMG6134) T

B. beta (LMG21987) T

B. yuanmingense (CCBAU10071) T

S. medicae (A321) T

M. mediterraneum (LMG17148) T

$M$. septentrionale (SDW014) T

$M$. temperatum (SDW018) T

$M$. thiogangeticum (SJT) T

$M$. tianshanense (A-1BS) T

WC12.1a $L$. sepiaria SA

WC19.1b L. cytisoides SA

WC19.1c $L$. cytisoides SA

WC23.1b $L$. multiflora SA

WC23.1C L. multiflora SA caggtgctgc atggctgtcg tcagctcgtg tcgtgagatg ttgggttaag caggtgctgc atggctgtcg tcagctcgtg tcgtgagatg ttgggttaag
1110
1120
1130
1140
1150

tcccgcaacg agcgcaaccc tcgccc-tta gttgccagc- at--tca--g tcccgcaacg agcgcaaccc tcgccc-tta gttgccagc- at--taa--g tcccgcaacg agcgcaaccc tcgccc-tta gttgccagc- at--tca--g tcccgcaacg agcgcaaccc tcgccc-tta gttgccagc- ct--tca--g tcccgcaacg agcgcaaccc tcgccc-tta gttgccagc- at--taa--g tcccgcaacg agcgcaaccc tcgccc-tta gttgccagc- at--tca--g tcccgcaacg agcgcaaccc tcgccc-tta gttgccagc- at--tca--g tcccgcaacg agcgcaaccc tcgccc-tta gttgccagc- at--tca--g tcccgcaacg agcgcaaccc tcgccc-tta gttgccagc- at--tca--g tcccgcaacg agcgcaaccc tcgcccttta gttgccagc- at--tca--g tcccgcaacg agcgcaaccc tcgccc-tta gttgccagc- at--tca--g tcccgcaacg agcgcaaccc tcgccc-tta gttgccagc- at--tca--g tcccgcaacg agcgcaaccc tcgccc-tta gttgccagc- at--taa--g tcccgcaacg agcgcaaccc tcgccc-tta gttgccagc- at--tca--g tcccgcaacg agcgcaaccc tcgccc-tta gttgccagc- at--tca--g tcccgcaacg agcgcaaccc tcgccc-tta gttgccagc- at--taa--g tcccgcaacg agcgcaaccc tcgccc-tta gttgccagc- at--taa--g tcccgcaacg agcgcaaccc tcgccc-tta gttgccagc- at--tca--g tcccgcaacg agcgcaaccc tcgccc-tta gttgccagc- at--taa--g tcccgcaacg agcgcaaccc tcgccc-tta gttgccagc- at--tta--g tcccgcaacg agcgcaaccc tcgccc-tta gttgccagc- at--tta--g tcccgcaacg agcgcaaccc tcgccc-tta gttgccagc- at--tta--g tcccgcaacg agcgcaaccc tcgccc-tta gttgccagc- at--tca--g tcccgcaacg agcgcaa-cc tcgccc-tta gttgccagc- at--tca--g tcccgcaacg agcgcaaccc tcgccc-tta gttgccagc- at--tca--g tcccgcaacg agcgcaaccc tcgccc-tta gttgccagc- at--taa--g tcccgcaacg agcgcaaccc tcgccc-tta gttgccagc- at--taa--g tcccgcaacg agcgcaaccc tcgccc-tta gttgccagc- at--taa--g tcccgcaacg agcgcaaccc tcgccc-tta gttgccagc- at--taa--g tcccgcaacg agcgcaaccc tcgccc-tta gttgccagc- at--taa--g tcccgcaacg agcgcaaccc tcgccc-tta gttgccagc- at--taa--g tcccgcaacg agcgcaaccc tcgcccttta gttgccagc- at--taa--g tcccgcaacg agcgcaaccc tcgccc-tta gttgccagc- at--taa--g tcccgcaacg agcgcaaccc ccgtcc-tta gttgctacc- at--tta--g tcccgcaacg agcgcaaccc ccgtcc-tta gttgctacc- at--tta--g tcccgcaacg agcgcaaccc ccgtcc-tta gttgctacc- at--tta--g tcccgcaacg agcgcaaccc ccgtcc-tta gttgctacc- at--tta--g tcccgcaacg agcgcaaccc ccgtcc-tta gttgctacc- at--tta--g tcccgcaacg agcgcaaccc ccgtcc-tta gttgctacc- at--tta--g tcccgcaacg agcgcaaccc ccgtcc-tta gttgctacc- at--tta--g tcccgcaacg agcgcaaccc ccgtcc-tta gttgctacc- at--tta--g tcccgcaacg agcgcaaccc ccgtcc-tta gttgctacc- at--tta--g tcccgcaacg agcgcaaccc ccgtcc-tta gttgctacc- at--tta--g tcccgcaacg agcgcaaccc tcgccc-tta gttgccatc- at--tga--g tcccgcaacg agcgcaaccc tcgcct-tta gttgccatc- at--tca--g tcccgcaacg agcgcaaccc tcatct-tca gttgccagc- aa--gtaacg tcccgcaacg agcgcaaccc tcatct-tca gttgccatc- at--tta--g tcccgcaacg agcgcaaccc ctaccg-cca gttgccatc- at--tca--g tcccgcaacg agcgcaaccc ctaccg-tca gttgccatc- at--tca--g tcccgcaacg agcgcaaccc acgtcc-tta gttgccatc- at--taa--g tcccgcaacg agcgcaaccc t-gtcc-tta gttgctacc- at--tta--g tcccgcaacg agcgcaaccc tcgccc-tta gttgccagc- at--taa--g tcccgcaacg agcgcaaccc tcgccc-tta gttgccagc- at--taa--g tcccgcaacg agcgcaaccc tcgccc-tta gttgccagc- at--tca--g tcccgcaacg agcgcaaccc tcgccc-tta gttgccagc- at--tca--g tcccgcaacg agcgcaaccc tcgccc-tta gttgccagc- at--tca--g tcccgcaacg agcgcaaccc tcgccc-tta gttgccatc- at--tca--g tcccgcaacg agcgcaaccc ccgtcc-tta gttgctacc- at--tta--g tcccgcaacg agcgcaaccc ccgtcc-tta gttgctacc- at--tta--g tcccgcaacg agcgcaaccc ccgtcc-tta gttgctacc- at--tta--g tcccgcaacg agcgcaaccc tcgccc-tta gttgccagc- at--tca--g

1160

1170

1180

1190

1200 ttgggcactc taaggggact gccggtgata agccgagagg aaggtgggga ttgggcactc taaggggact gccggtgata agccgagagg aaggtgggga ttgggcactc taaggggact gccggtgata agccgagagg aaggtgggga ttgggcactc taaggggact gccggtgata agccgagagg aaggtgggga ttgggcactc taaggggact gccggtgata agccgagagg aaggtgggga ttgggcactc taaggggact gccggtgata agccgagagg aaggtgggga ttgggcactc taaggggact gccggtgata agccgagagg aaggtgggga ttgggcactc taaggggact gccggtgata agccgagagg aaggtgggga ttgggcactc taaggggact gccggtgata agccgagagg aaggtgggga ttgggcactc taaggggact gccggtgata agccgagagg aaggtgggga 
WC23.1d $L$. multiflora SA WC28.1F $L$. sericea SA WC28.1g $L$. sericea SA WC28.1h $L$. sericea SA WC28.1i L. sericea SA WC33b L. sericea SA WC33C $L$. sericea SA WC33e $L$. sericea SA WC33h L. sericea SA

$S$. americanum (CFNEI156) T

$S$. arboris (LMG14919) T

S. fredii (LMG6217) T

S. kostiense (LMG19227)

S. kummerowiae (CCBAU71714) T

S. meliloti (LMG6133) T

BH1LSC $L$. spinescens SA

BH2LSa $L$. spinescens SA

BH3LSd L. spinescens SA

BW1LSab $L$. spinescens SA

BW2LSa $L$. spinescens SA

BW2LSb $L$. spinescens SA

BW2LSC $L$. spinescens SA

BW3LSd $L$. spinescens SA

$B$. canariense (MSFC2-M3-R-3) T

$B$. japonicum (LMG6138) T

$B$. liaoningense (LMG18230) T

Betal106d $L$. pungens SA

Betal106a $L$. pungens SA

NK22 $L$. sericea SA

WC21.1L $L$. pauciflora SA

XHRla $L$. simsiana SA

Betal106f $L$. pungens SA

$R$. palustris (ATCC17001)

$M$. nodulans (ORS2060)

Az. caulinodans (LMG6465)

R. rubrum (LMGATCC11170)

A. magnetotacticum (M58171)

Az. brasilense (NCIMB11860)

Az. Iipoferum (NCIMB11861)

R. acidophila (M34128)

A. clevelandensis (M69186)

$S$. morelense (LMG21331) T

$M$. amorphae (LMG18977) T

M. ciceri (LMG14989) T

$M$. huakuii (LMG14107) T

M. loti (LMG6125) T

M. plurifarium (LMG11892) T

B. elkanii (LMG6134) T

B. beta (LMG21987) T

B. yuanmingense (CCBAU10071) T

S. medicae (A321) T

M. mediterraneum (LMG17148) T

$M$. septentrionale (SDW014) T

M. temperatum (SDW018) T

$M$. thiogangeticum (SJT) T

$M . t i a n s h a n e n s e$ (A-1BS) T

WC12.1a L. sepiaria SA

WC19.1b L. cytisoides SA

WC19.1C $L$. cytisoides SA

WC23.1b L. multiflora SA

WC23.1C $L$. multiflora SA

WC23.1d I multiflora SA

WC28.1F $L$. sericea SA

WC28.1g L. sericea SA

WC28.1h $L$. sericea SA

WC28.1i $L$. sericea SA

WC33b L. sericea SA

WC33c $L$. sericea SA

WC33e $L$. sericea SA

WC33h $L$. sericea SA

S. americanum (CFNEI156) T

S. arboris (LMG14919) T

S. fredii (LMG6217) T

S. kostiense (LMG19227)

S. kummerowiae (CCBAU71714) T ttgggcactc taaggggact gccggtgata agccgagagg aaggtgggga ttgggcactc taaggggact gccggtgata agccgagagg aaggtgggga ttgggcactc taaggggact gccggtgata agccgagagg aaggtgggga ttgggcactc taaggggact gccggtgata agccgagagg aaggtgggga ttgggcactc taaggggact gccggtgata agccgagagg aaggtgggga ttgggcactc taaggggact gccggtgata agccgagagg aaggtgggga ttgggcactc taaggggact gccggtgata agccgagagg aaggtgggga ttgggcactc taaggggact gccggtgata agccgagagg aaggtgggga ttgggcactc taaggggact gccggtgata agccgagagg aaggtgggga ttgggcactc taaggggact gccggtgata agccgagagg aaggtgggga ttgggcactc taaggggact gccggtgata agccgagagg aaggtgggga ttgggcactc taaggggact gccggtgata agccgagagg aaggtgggga ttgggcactc taaggggact gccggtgata agccgagagg aaggtgggga ttgggcactc taaggggact gccggtgata agccgagagg aaggtgggga ttgggcactc taaggggact gccggtgata agccgagagg aaggtgggga ttgggcactc taaggggact gccggtgata agccgagagg aaggtgggga ttgggcactc taaggggact gccggtgata agccgagagg aaggtgggga ttgggcactc taaggggact gccggtgata agccgagagg aaggtgggga ttgggcactc taaggggact gccggtgata agccgagagg aaggtgggga ttgggcactc taaggggact gccggtgata agccgagagg aaggtgggga ttgggcactc taaggggact gccggtgata agccgagagg aaggtgggga ttgggcactc taaggggact gccggtgata agccgagagg aaggtgggga ttgggcactc taaggggact gccggtgata agccgagagg aaggtgggga ttgagcactc taaggagact gccggtgata agccgcgagg aaggtgggga ttgagcactc taaggagact gccggtgata agccgcgagg aaggtgggga ttgagcactc taaggagact gccggtgata agccgcgagg aaggtgggga ttgagcactc taaggagact gccggtgata agccgcgagg aaggtgggga ttgagcactc taaggagact gccggtgata agccgcgagg aaggtgggga ttgagcactc taaggagact gccggtgata agccgcgagg aaggtgggga ttgagcactc taaggagact gccggtgata agccgcgagg aaggtgggga ttgagcactc taaggagact gccggtgata agccgcgagg aaggtgggga ttgagcactc taaggagact gccggtgata agccgcgagg aaggtgggga ttgagcactc taaggagact gccggtgata agccgcgagg aaggtgggga ttgggcactc tagggggact gccggtgata agccgcgagg aaggtgggga ttgggcactc taaagggact gccggtgata agccgcgagg aaggtgggga ttgggcactc tgaagagact gccggtgaca agccg-gagg aaggtgggga ttgggcacgc tgaagaaact gccggtgaca agcc-ggagg aaggtgggga ttgggcactc tggtggaact gccggtgaca agccg-gagg aaggcgggga ttgggcactc tggtggaacc gccggtgaca agccg-gagg aaggcgggga ttgggcactc tagggagact gccggtgata agccgagagg aaggtgtgga ttgagcactc taaggagact gccggtgata agccgcgagg aaggtgggga ttgggcactc taaggggact gccggtgata agccgagagg aaggtgggga ttgggcactc taaggggact gccggtgata agccgagagg aaggtgggga ttgggcactc taaggggact gccggtgata agccgagagg aaggtgggga ttgggcactc taaggggact gccggtgata agccgagagg aaggtgggga ttgggcactc taaggggact gccggtgata agccgagagg aaggtgggga ttgggcactc taaggggact gccggtgata agccgagagg aaggtgggga ttgagcactc taaggagact gccggtgata agccgcgagg aaggtgggga ttgagcactc taaggagact gccggtgata agccgcgagg aaggtgggga ttgagcactc taaggagact gccggtgata agccgcgagg aaggtgggga ttgggcactc taaggggact gccggtgata agccgagagg aaggtgggga

1210

1220

1230

1240

1250

tgacgtcaag tcctcatggc ccttacgggc tgggc-taca cacgtgctac tgacgtcaag tcctcatggc ccttacgggc tgggc-taca cacgtgctac tgacgtcaag tcctcatggc ccttacgggc tgggc-taca cacgtgctac tgacgtcaag tcctcatggc ccttacggga tgggcttaca cacgtgctac tgacgtcaag tcctcatggc ccttacgggc tgggc-taca cacgtgctac tgacgtcaag tcctcatggc ccttacgggc tgggc-taca cacgtgctac tgacgtcaag tcctcatggc ccttacgggc tgggc-taca cacgtgctac tgacgtcaag tcctcatggc ccttacgggc tgggc-taca cacgtgctac tgacgtcaag tcctcatggc ccttacgggc tgggc-taca cacgtgctac tgacgtcaag tcctcatggc ccttacgggc tgggc-taca cacgtgctac tgacgtcaag tcctcatggc ccttacgggc tgggc-taca cacgtgctac tgacgtcaag tcctcatggc ccttacgggc tgggc-taca cacgtgctac tgacgtcaag tcctcatggc ccttacgggc tgggc-taca cacgtgctac tgacgtcaag tcctcatggc ccttacgggc tgggc-taca cacgtgctac tgacgtcaag tcctcatggc ccttacgggc tgggc-taca cacgtgctac tgacgtcaag tcctcatggc ccttacgggc tgggc-taca cacgtgctac tgacgtcaag tcctcatggc ccttacgggc tgggc-taca cacgtgctac tgacgtcaag tcctcatggc ccttacgggc tgggc-taca cacgtgctac tgacgtcaag tcctcatggc ccttacgggc tgggc-taca cacgtgctac tgacgtcaag tcctcatggc ccttacgggc tgggc-taca cacgtgctac tgacgtcaag tcctcatggc ccttacgggc tgggc-taca cacgtgctac tgacgtcaag tcctcatggc ccttacgggc tgggc-taca cacgtgctac tgacgtcaag tcctcatggc ccttacgggc tgggc-taca cacgtgctac tgacgtcaag tcctcatggc ccttacgggc tgggc-taca cacgtgctac 
S. meliloti (LMG6133) T BH1LSC L. spinescens SA BH2LSa $L$. spinescens SA BH3LSd $L$. spinescens SA BW1LSab $L$. spinescens SA BW2LSa $L$. spinescens SA BW2LSb $L$. spinescens SA BW2LSC $L$. spinescens SA BW3LSd $L$. spinescens $S A$

B. canariense (MSFC2-M3-R-3) T

B. japonicum (LMG6138) T

$B$. liaoningense (LMG18230) T

Betallo6d L. pungens SA

Betall06a L. pungens SA

NK22 L. sericea SA

WC21.1L $L$. pauciflora SA

XHR1a L. simsiana SA

Betal106f L. pungens SA

R. palustris (ATCC17001)

$M$. nodulans (ORS2060)

Az. Caulinodans (LMG6465)

$R$. rubrum (LMGATCC11170)

A. magnetotacticum (M58171)

Az. brasilense (NCIMB11860)

$A z$. lipoferum (NCIMB11861)

$R$. acidophila (M34128)

A. clevelandensis (M69186)

S. morelense (LMG21331) T

M. amorphae (LMG18977) T

M. ciceri (LMG14989) T

M. huakuii (LMG14107) T

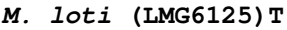

$M$. plurifarium (LMG11892) T

B. elkanii (LMG6134)T

B. beta (LMG21987) T

B. yuanmingense (CCBAU10071) T

S. medicae (A321) T

M. mediterraneum (LMG17148) T

$M$. septentrionale (SDW014) T

$M$. temperatum (SDW018) T

$M$. thiogangeticum (SJT) T

$M . t i a n s h a n e n s e$ (A-1BS) T

WC12.1a $L$. sepiaria SA

WC19.1b $L$. cytisoides SA

WC19.1C L. cytisoides SA

WC23.1b L. multiflora SA

WC23.1C L. multiflora SA

WC23.1d L. multiflora SA

WC28.1F $L$. sericea SA

WC28.1g $L$. sericea SA

WC28. $1 \mathrm{~h}$ L sericea SA

WC28.1i $L$. sericea SA

WC33b $L$. sericea SA

WC33C $L$. sericea SA

WC33e $L$. sericea SA

WC33h $L$. sericea SA

$S$. americanum (CFNEI156) T

S. arboris (LMG14919) T

S. fredii (LMG6217) T

S. kostiense (LMG19227)

S. kummerowiae (CCBAU71714) T

S. meliloti (LMG6133) T

BH1LSc $L$. spinescens SA BH2LSa $L$. spinescens SA BH3LSd L. spinescens SA BW1LSab $L$. spinescens SA BW2LSa $L$. spinescens SA BW2LSb $L$. spinescens SA BW2LSC $L$. spinescens SA BW3LSd $L$. spinescens SA $B$. canariense (MSFC2-M3-R-3) T

B. japonicum (LMG6138) T

B. liaoningense (LMG18230) T

Betall06d L. pungens SA

Betal106a $L$. pungens SA tgacgtcaag tcctcatggc ccttacgggc tgggc-taca cacgtgctac tgacgtcaag tcctcatggc ccttacgggc tgggc-taca cacgtgctac tgacgtcaag tcctcatggc ccttacgggc tgggc-taca cacgtgctac tgacgtcaag tcctcatggc ccttacgggc tgggc-taca cacgtgctac tgacgtcaag tcctcatggc ccttacgggc tgggc-taca cacgtgctac tgacgtcaag tcctcatggc ccttacgggc tgggc-taca cacgtgctac tgacgtcaag tcctcatggc ccttacgggc tgggc-taca cacgtgctac tgacgtcaag tcctcatggc ccttacgggc tgggc-taca cacgtgctac tgacgtcaag tcctcatggc ccttacgggc tgggc-taca cacgtgctac tgacgtcaag tcctcatggc ccttacgggc tgggc-taca cacgtgctac tgacgtcaag tcctcatggc ccttacgggc tgggc-taca cacgtgctac tgacgtcaag tcctcatggc ccttacgggc tgggc-taca cacgtgctac tgacgtcaag tcctcatggc ccttacgggc tgggc-taca cacgtgctac tgacgtcaag tcctcatggc ccttacgggc tgggc-taca cacgtgctac tgacgtcaag tcctcatggc ccttacgggc tgggc-taca cacgtgctac tgacgtcaag tcctcatggc ccttacgggc tgggc-taca cacgtgctac tgacgtcaag tcctcatggc ccttacgggc tgggc-taca cacgtgctac tgacgtcaag tcctcatggc ccttacgggc tgggc-taca cacgtgctac tgacgtcaag tcctcatggc ccttacgggc tgggc-taca cacgtgctac tgacgtcaag tcctcatggc ccttacggga tgggc-taca cacgtgctac tgacgtcaag tcctcatggc ccttacgggc tgggc-taca cacgtgctac tgacgtcaag tcctcatggc ccttacgggc tgggc-taca cacgtgctac tgacgtcaag tcctcatgcg --ttacgggc tgggc-taca cacgtgctac tgacgtcaag tcctcatggc ccttatgggt tgggc-taca cacgtgctac tgacgtcaag tcctcatggc ccttatgggt tgggc-taca cacgtgctac tgacgtcaag tcctcatggc ccttacaggc tgggc-taca cacgtgctac tgacgtcaag tcctcatggc ccttacgggc tgggc-taca cacgtgctac tgacgtcaag tcctcatggc ccttacgggc tgggc-taca cacgtgctac tgacgtcaag tcctcatggc ccttacgggc tgggc-taca cacgtgctac tgacgtcaag tcctcatggc ccttacgggc tgggc-taca cacgtgctac tgacgtcaag tcctcatggc ccttacgggc tgggc-taca cacgtgctac tgacgtcaag tcctcatggc ccttacgggc tgggc-taca cacgtgctac tgacgtcaag tcctcatggc ccttacgggc tgggc-taca cacgtgctac tgacgtcaag tcctcatggc ccttacgggc tgggc-taca cacgtgctac tgacgtcaag tcctcatggc ccttacgggc tgggc-taca cacgtgctac tgacgtcaag tcctcatggc ccttacgggc tgggc-taca cacgtgctac tgacgtcaag tcctcatggc ccttacgggc tgggc-taca cacgtgctac 1270 1280 aatggtggtg acagtgggca gcgagaccgc gaggtcgagc taatctccaa aatggtggtg acagtgggca gcgagaccgc gaggtcgagc taatctccaa aatggtggtg acagtgggca gcgagaccgc gaggtcgagc taatctccaa aatggtggtg acagtgggca gcgagaccgc gaggtcgagc taatctccaa aatggtggtg acagtgggca gcgagaccgc gaggtcgagc taatctccaa aatggtggtg acagtgggca gcgagaccgc gaggtcgagc taatctccaa aatggtggtg acagtgggca gcgagaccgc gaggtcgagc taatctccaa aatggtggtg acagtgggca gcgagaccgc gaggtcgagc taatctccaa aatggtggtg acagtgggca gcgagaccgc gaggtcgagc taatctccaa aatggtggtg acagtgggca gcgagaccgc gaggtcgagc taatctccaa aatggtggtg acagtgggca gcgagaccgc gaggtcgagc taatctccaa aatggtggtg acagtgggca gcgagaccgc gaggtcgagc taatctccaa aatggtggtg acagtgggca gcgagaccgc gaggtcgagc taatctccaa aatggtggtg acagtgggca gcgagaccgc gaggtcgagc taatctccaa aatggtggtg acagtgggca gcgagaccgc gaggtcgagc taatctccaa aatggtggtg acagtgggca gcgagaccgc gaggtcgagc taatctccaa aatggtggtg acagtgggca gcgagaccgc gaggtcgagc taatctccaa aatggtggtg acagtgggca gcgagaccgc gaggtcgagc taatctccaa aatggtggtg acagtgggca gcgagaccgc gaggtcgagc taatctccaa aatggtggtg acagtgggca gcgagaccgc gaggtcgagc taatctccaa aatggtggtg acagtgggca gcgagaccgc gaggtcgagc taatctccaa aatggtggtg acagtgggca gcgagaccgc gaggtcgagc taatctccaa aatggtggtg acagtgggca gcgagaccgc gaggtcgagc taatctccaa aatggtggtg acagtgggca gcgagaccgc gaggtcgagc taatctccaa aatggtggtg acagtgggca gcgagaccgc gaggtcgagc taatctccaa aatggtggtg acagtgggca gcgagaccgc gaggtcgagc taatctccaa aatggtggtg acagtgggca gcgagaccgc gaggtcgagc taatctccaa aatggtggtg acagtgggca gcgagaccgc gaggtcgagc taatctccaa aatggtggtg acagtgggca gcgagaccgc gaggtcgagc taatctccaa aatggtggtg acagtgggca gcgagaccgc gaggtcgagc taatctccaa aatggtggtg acagtgggca gcgagaccgc gaggtcgagc taatctccaa aatggtggtg acagtgggca gcgagaccgc gaggtcgagc taatctccaa aatggtggtg acagtgggca gcgagaccgc gaggtcgagc taatctccaa aatggcggtg acaatgggat gcgaagacgc aagtcctggc aaatctcaaa aatggcggtg acaatgggat gctaaggggc gacccttcgc aatctcaaa aatggcggtg acaatgggat gctaaggggc gacccttcgc aaatctcaaa aatggcggtg acaatgggat gctaaggggt gacccttcgc aaatctcaaa aatggcggtg acaatgggat gctaaggggc gacccttcgc aaatctcaaa 
NK22 $L$. sericea SA WC21.1L L. pauciflora SA XHRla $L$. simsiana SA Betal106f $L$. pungens SA $R$. palustris (ATCC17001) $M$. nodulans (ORS2060)

$A z$. Caulinodans (LMG6465)

$R$. rubrum (LMGATCC11170)

A. magnetotacticum (M58171)

Az. brasilense (NCIMB11860)

Az. Iipoferum (NCIMB11861)

R. acidophila (M34128)

A. clevelandensis (M69186)

S. morelense (LMG21331) T

$M$. amorphae (LMG18977) T

M. ciceri (LMG14989) T

M. huakuii (LMG14107) T

M. loti (LMG6125) T

M. plurifarium (LMG11892) T

B. elkanii (LMG6134) T

B. beta (LMG21987) T

B. yuanmingense (CCBAU10071) T

S. medicae (A321) T

M. mediterraneum (LMG17148) T

$M$. septentrionale (SDW014)T

$M$. temperatum (SDW018) T

$M$. thiogangeticum (SJT) T $M . t i a n s h a n e n s e$ (A-1BS) T WC12.1a $L$. sepiaria SA

WC19.1b L. cytisoides SA WC19.1C L. cytisoides SA WC23.1b $L$. multiflora SA WC23.1C $L$. multiflora SA WC23.1d L. multiflora SA WC28.1F $L$. sericea SA WC28.1g $L$. sericea SA WC28.1h $L$. sericea SA WC28.1i $L$. sericea SA WC33b L. sericea SA WC33C I, sericea SA WC33e $L$, sericea SA WC33h $L$. sericea SA $S$. americanum (CFNEI156) T

S. arboris (LMG14919) T

S. fredii (LMG6217) T

S. kostiense (LMG19227)

S. kummerowiae (CCBAU71714) T

S. meliloti (LMG6133) T

BH1LSC $L$. spinescens SA BH2LSa $L$. spinescens SA BH3LSd $L$ spinescens SA BW1LSab $L$. spinescens SA BW2LSa L. spinescens SA BW2LSb $L$. spinescens SA BW2LSC $L$. spinescens SA BW3LSd $L$. spinescens SA $B$. canariense (MSFC2-M3-R-3) T B. japonicum (LMG6138) T B. liaoningense (LMG18230) T Betal106d L. pungens SA Betal106a L. punges SA NK22 $L$. sericea SA WC21.1L $L$. pauciflora SA XHR1a L. simsiana SA Betal106f $L$. pungens SA $R$. palustris (ATCC17001) $M$. nodulans (ORS2060)

Az. caulinodans (LMG6465)

R. rubrum (LMGATCC11170)

A. magnetotacticum (M58171)

Az. brasilense (NCIMB11860)

Az. Iipoferum (NCIMB11861)

R. acidophila (M34128)

A. clevelandensis (M69186)

S. morelense (LMG21331) T aatggcggtg acaatgggat gctaaggggc gacccttcgc aaatctcaaa aatggcggtg acaatgggat gctaaggggc gacccttcgc aaatctcaaa aatggcggtg acaatgggac gctaaggggc aacccttcgc aaatctcaaa aatggcggtg acaatgggat gctaaggggt gacccttcgc aaatctcaaa aatggcggtg acaatgggaa gctaaggggc gacccttcgc aaatctcaaa aatggcggtg acaatgggaa gcgaaggggc gacctggagc aaatccccaa aatggcggtg acaatgggat gcgagcctgc gagggtgagc aatctccaa aatggcgcct acaatgggca gcgacctcgc gaggggaagc taatctccaa aatggtggtg acagtgggts sctaactcgc gaggtatg-c taatccctaa aatggcggtg acagtgggat gcgaagtcgc aagatggagc caatccccaa aatggcggtg acagtgggaa gcgaagtcgc gagatggagc caatccccaa aatggcggtg acaatgggaa gcgaaagggc gacctctagc aatctcaaa aatggcggtg acaatggga- gcaaaggggc gacccctagc aatctcaaa aatggtggtg acagtgggca gcgagaccgc gaggtcgagc taatctccaa aatggtggtg acagtgggca gcgagaccgc gaggtcgagc taatctccaa aatggtggtg acagtgggca gcgagaccgc gaggtcgagc taatctccaa aatggtggtg acagtgggca gcgagaccgc gaggtcgagc taatctccaa aatggtggtg acagtgggca gcgagaccgc gaggtcgagc taatctccaa aatggtggtg acagtgggca gcgagaccgc gaggtcgagc taatctccaa aatggcggtg acaatgggat gctaaggggc gacccttcgc aaatctcaaa aatggcggtg acaatgggat gctaaggggc gacccttcgc aaatctcaaa aatggcggtg acaatgggat gctaaggggc gacccttcgc aaatctcaaa aatggtggtg acagtgggca gcgagaccgc gaggtcgagc taatctccaa

1310 1320 1330 1340 1350 aagcc-atct cagttcggat tgcactctgc aactcgagtg catgaagttg aagcc-atct cagttcggat tgcactctgc aactcgagtg catgaagttg aagcc-atct cagttcggat tgcactctgc aactcgagtg catgaagttg gagcc-atct cagttcggat tgcactctgc aactcgagtg catgaagttg aagcc-atct cagttcggat tgcactctgc aactcgagtg catgaagttg aagcc-atct cagttcggat tgcactctgc aactcgagtg catgaagttg aagcc-atct cagttcggat tgcactctgc aactcgagtg catgaagttg aagcc-atct cagttcggat tgcactctgc aactcgagtg catgaagttg aagcc-atct cagttcggat tgcactctgc aactcgagtg catgaagttg aagcc-atct cagttcggat tgcactctgc aactcgagtg catgaagttg aagcc-atct cagttcggat tgcactctgc aactcgagtg catgaagttg aagcc-atct cagttcggat tgcactctgc aactcgagtg catgaagttg aagcc-atct cagttcggat tgcactctgc aactcgagtg catgaagttg aagcc-atct cagttcggat tgcactctgc aactcgagtg catgaagttg aagcc-atct cagttcggat tgcactctgc aactcgagtg catgaagttg aagcc-atct cagttcggat tgcactctgc aactcgagtg catgaagttg aagcc-atct cagttcggat tgcactctgc aactcgagtg catgaagttg aagcc-atct cagttcggat tgcactctgc aactcgagtg catgaagttg aagcc-atct cagttgggat tgcactctgc aactcgagtg catgaagttg aagcc-atct cagttcggat tgcactctgc aactcgagtg catgaagtto aagcc-atct cagttcggat tgcactctgc aactcgagtg catgaagttg aagcc-atct cagttcggat tgcactctgc aactcgagtg catgaagttg aagcc-atct cagttcggat tgcactctgc aactcgggtg catgaagttg aagcc-atct cagttcggat tgcactctgc aactcgagtg catgaagttg aagcc-atct cagttcggat tgcactctgc aactcgagtg catgaagttg aagcc-atct cagttcggat tgcactctgc aactcgagtg catgaagttg aagcc-atct cagttcggat tgcactctgc aactcgagtg catgaagttg aagcc-atct cagttcggat tgcactctgc aactcgagtg catgaagttg aagcc-atct cagttcggat tgcactctgc aactcgagtg catgaagttg aagcc-atct cagttcggat tgcactctgc aactcgagtg catgaagttg aagcc-atct cagttcggat tgcactctgc aactcgagtg catgaagttg aagcc-atct cagttcggat tgcactctgc aactcgagtg catgaagttg aagcc-atct cagttcggat tgcactctgc aactcgagtg catgaagttg aagcc-gtct cagttcggat tgggctctgc aactcgagcc catgaa---aagcc-gtct cagttcggat tgggctctgc aactcgagcc catgaagttg aagcc-gtct cagttcggat tgggctctgc aactcgagcc catgaagttg aagcc-gtct cagttcggat tgggctctgc aactcgagcc catgaagttg aagcc-gtct cagttcggat tgggctctgc aactcgagcc catgaagttg aagcc-gtct cagttcggat tgggctctgc aactcgagcc catgaagttg aagcc-gtct cagttcggat tgggctctgc aactcgagcc catgaagttg aagcc-gtct cagttcggat tgggctctgc aactcgagcc catgaagttg aagcc-gtct cagttcggat tgggctctgc aactcgagcc catgaagttg aagcc-gtct cagttcggat tgggctctgc aactcgagcc catgaagttg aagcc-gtct cagttcagat tgcactctgc aactcgagtg catgaaggcg aagcc-gtct cagttcggat tgcactctgc aactcgagtg catgaagttg aaggc-gtct cagttcggat tgcactctgc aactcgggtg catgaagtcg aagcc-atct cagttcggat tgcactctgc aactcgagtg catgaagtcg aagcc-gtct cagttcggat tgcactctgc aactcgggtg catgaagttg aagcc-gtct cagttcggat tgcactctgc aactcgagtg catgaagttg aaacc-gtct cagttcggat tgtactctgc aactcgagtg catgaaggtg aagcc-gtct cagttcggat tgggctctgc aactcgagcc catgaagttg aagcc-atct cagttcggat tgcactctgc aactcgagtg catgaagttg 
M. amorphae (LMG18977)T

M. ciceri (LMG14989)T

M. huakuii (LMG14107) T

M. loti (LMG6125) T

M. plurifarium (LMG11892) T

B. elkanii (LMG6134) T

B. beta (LMG21987) T

B. yuanmingense (CCBAU10071) T

S. medicae (A321) T

M. mediterraneum (LMG17148) T

M. septentrionale (SDW014) T

$M$. temperatum (SDW018) T

$M$. thiogangeticum (SJT) T M.tianshanense (A-1BS) T WC12.1a L. sepiaria SA

WC19.1b L. cytisoides SA WC19.1c $L$. cytisoides SA WC23.1b $L$. multiflora SA WC23.1c $L$. multiflora SA WC23.1d $L$. multiflora SA WC28.1F $L$, sericea SA WC28.1g $L$. sericea SA WC28.1h L. sericea SA WC28.1i L. sericea SA WC33b $L$. sericea SA WC33c $L$. sericea SA WC33e $L$. sericea SA WC33h L. sericea SA

$S$. americanum (CFNEI156) T

$S$. arboris (LMG14919) T

$S$. fredii (LMG6217) T

S. kostiense (LMG19227)

S. kummerowiae (CCBAU71714) T

S. meliloti (LMG6133) T

BH1LSC L. spinescens SA BH2LSa L. spinescens SA BH3LSd $L$. spinescens SA BW1LSab $L$. spinescens SA BW2LSa L. spinescens SA BW2LSb $L$. spinescens SA BW2LSC $L$. spinescens SA BW3LSd $L$. spinescens SA B. canariense (MSFC2-M3-R-3) T B. japonicum (LMG6138) T $B$. liaoningense (LMG18230) T Betal106d L. pungens SA Betal106a L. pungens SA NK22 $L$. sericea SA WC21.1L $L$. pauciflora SA XHR1a L. simsiana SA Betal106f L. pungens SA $R$. palustris (ATCC17001) $M$. nodulans (ORS2060)

Az. Caulinodans (LMG6465)

$R$. rubrum (LMGATCC11170)

A. magnetotacticum (M58171)

Az. brasilense (NCIMB11860)

Az. lipoferum (NCIMB11861)

R. acidophila (M34128)

A. Clevelandensis (M69186)

S. morelense (LMG21331) T

M. amorphae (LMG18977)T

M. Ciceri (LMG14989) T

$M$. huakuii (LMG14107)T

M. loti (LMG6125) T

M. plurifarium (LMG11892) T

B. elkanii (LMG6134) T

B. beta (LMG21987) T

B. yuanmingense (CCBAU10071) T

S. medicae (A321) T

M. mediterraneum (LMG17148) T

M. septentrionale (SDW014) T

$M$. temperatum (SDW018) T aagcc-atct cagttcggat tgcactctgc aactcgagtg catgaagttg aagcc-atct cagttcggat tgcactctgc aactccagtg catgaagttg aagcc-atct cagttcggat tgcactctgc aactcgagtg catgaagttg aagcc-atct cagttcggat tgcactctgc aactcgagtg catgaagttg aagcc-atct cagttcggat tgcactctgc aactcgagtg catgaagttg aagcc-gtct cagttcggat tgggctctgc aactcgagcc catgaagttg aagcc-gtct cagttcggat tgggctctgc aactcgagcc catgaagttg aagcc-gtct cagttcggat tgggctctgc aactcgagcc catgaagttg aagcc-atct cagttcggat tgcactctgc aactcgagtg catgaagttg

1360 1370

1380

1390

1400

gaatcgctag taatcgcgga tcagcatgcc gcggtgaata cgttcccggg gaatcgctag taatcgcgga tcagcatgcc gcggtgaata cgttcccggg gaatcgctag taatcgcgga tcagcatgcc gcggtgaata cgttcccggg gaatcgctag taatcgcgga tcagcatgcc gcggtgaata cgttcccggg gaatcgctag taatcgcgga tcagcatgcc gcggtgaata cgttcccggg gaatcgctag taatcgcgga tcagcatgcc gcggtgaata cgttcccggg gaatcgctag taatcgcgga tcagcatgcc gccaaggata cgttcccggg gaatcgctag taatcgcgga tcagcatccc gcagtgaata cgttcccggg gaatcgctag taatcgcgga tcagcatgcc gcggtgaata cgttcccggg gaatcgctag taatcgcgga tcagcatgcc gcagtgaata cgttcccggg gaatcgctag taatcgcgga tcagcatgcc gcggtgaata cgttcccggg gaatcgctag taatcgcgga tcagcatgcc gcggtgaata cgttcccggg gaatcgctag taatcgcgga tcagcatgcc gcggtgaata cgttcccggg gaatcgctag taatcgcgga tcagcatgcc gcgatgaata cgttcccggg gaatcgctag taatcgcgga tcagcatgcc gcggtgaata cgttcccggg gaatcgctag taatcgcgga tcagcatgcc gcggtgaata cgttcccggg gaatcgctag taatcgcgga tcagcatgcc gcagtgaata cgttcccggg gaatcgctag taatcgcgga tcagcatgcc gcggtgaata cgttcccggg gaatcgctag taatcgcgga tg-

gaatcgctag taatcgcaga tcagcatgct gcggtgaata cgttcccggg gaatcgctag taatcgcaga tcagcatgct gcggtgaata cgttcccggg gaatcgctag taatcgcaga tcagcatgct gcggtgaata cgttcccggg gaatcgctag taatcgcaga tcagcatgct gcggtgaata cgttcccggg gaatcgctag taatcgcaga tcagcatgcc gcggtgaata cgttcccggg gaatcgctag taatcgcaga tcagcatgct gcggtgaata cgttcccggg gaatcgctag taatcgcaga tcagcatgct gcggtgaata cgttcccggg gaatcgctag taatcgcaga tcagcatgct gcggtgaata cgttcccggg gaatcgctag taatcgcaga tcagcatgct gcggtgaata cgttcccggg gaatcgctag taatcgcaga tcagcatgct gcggtgaata cgttcccggg gaatcgctag taatcgcaga tcagcatgct gcggtgaata cgttcccggg gaatcgctag taatcgcaga tcagcatgct gcggtgaata cgttcccggg gaatcgctag taatcgcaga tcagcatg-t gaggtgaata cgttcccggg gaatcgctag taatcgcaga tcagcatgct gcggtgaata cgttcccggg

gaatcgctag taatcgtgga tcagcacgcc acggtgaata cgttcccggg gaatcgctag taatcgtgga tcagcacgcc acggtgaata cgttcccggg gaatcgctag taatcgtgga tcagcacgcc acaatgaata cgttcccggg gaatcgctag taatcgtgga tcagcacgcc acggtgaata cgttcccggg gaatcgctag taatcgtgga tcagcacgcc acggtgaata cgttcccggg gaatcgctag taatcgtgga tcagcacgcc acggtgaata cgttcccggg gaatcgctag taatcgtgga tcagcacgcc acggtgaata cgttcccggg gaatcgctag taatcgtgga tcagcacgcc actgtgaata cgttcccggg gaatcgctag taatcgtgga tcagcatgcc acggtgaata cgt------gaatcgctag taatcgtgga tcagcacgcc acggtgaata cgttcccggg gaatcgctag taatcgtgga tcagcatgcc acggtgaata cgttcccggg gaatcgctag taatcgtgga tcagcatgcc acggtgaata cgttcccggg gaatcgctag taatcgtgga tcagcatgcc acggtgaata cgttcccggg gaatcgctag taatcgcgga tcagcacgcc gcggtgaata cgttcccggg gaatcgctag taatcgcgga tcagcacgcc gcggtgaata cgttcccggg gaatcgctag taatcgcaga tcagaacgct gcggtgaata cgttcccggg gaatcgctag taatcgtgga tcagcatgcc acggtgaata cgttcccggg gaatcgctag taatcgcaga tcagcatgct gcggtgaata cgttcccggg gaatcgctag taatcgcgga tcagcatgcc gcggtgaata cgttcccggg gaatcgeta-

gaatcgctag taatcgcgga tcagcatgcc gcggtgaata cgttcccggg gaatcgctag taatcgcgga tcagcatgcc gcggtgaata cgttcccggg gaatcgctag taatcgcgga tcagcatgcc gcggtgaata cgttcccggg gaatcgctag taatcgtgga tcagcacgcc acggtgaata cgttcccggg gaatcgctag taatcgtgga tcagcacgcc acggtgaata cgttcccggg gaatcgctag taatcgtgga tcagcacgcc acggtgaata cgttcccggg gaatcgctag taatcgcaga tcagcatgct gcggtgaata cgttcccggg

1410

1420

1430

1440

1450

ccttgtacac accgcccgtc acaccatggg agttggtttt acccoaaggc ccttgtacac accgcccgtc acaccatggg agttggtttt acccgaaggc ccttgtacac accgcccgtc acaccatggg agttggtttt acccgaaggc 
M. thiogangeticum (SJT) T $M . t i a n s h a n e n s e$ (A-1BS) T WC12.1a $L$. sepiaria SA WC19.1b $L$. cytisoides SA WC19.1C L. cytisoides SA WC23.1b $L$. multiflora SA WC23.1c $L$. multiflora SA WC23.1d L. multiflora SA WC28.1F $L$. sericea SA WC28.1g $L$. sericea SA WC28.1h $L$. sericea SA WC28.1i $L$. sericea SA WC33b L. sericea SA WC33c L. sericea SA WC33e $L$. sericea SA WC33h $L$. sericea SA $S$. americanum (CFNEI156) T

$S$. arboris (LMG14919) T

$S$. fredii (LMG6217) T

S. kostiense (LMG19227)

S. kummerowiae (CCBAU71714) T S. meliloti (LMG6133) T BH1LSC $L$. spinescens SA BH2LSa $L$. spinescens SA BH3LSd $L$. spinescens SA BW1LSab $L$. spinescens SA BW2LSa $L$. spinescens SA BW2LSb $L$. spinescens SA BW2LSC $L$. spinescens SA BW3LSd $L$. spinescens SA $B$. canariense (MSFC2-M3-R-3) T B. japonicum (LMG6138) T B. liaoningense (LMG18230) T Betal106d L. pungens SA Betal106a L. pungens SA NK22 L. sericea SA WC21.1L $L$. pauciflora SA XHRla $L$. simsiana SA Betal106f $L$. pungens SA $R$. palustris (ATCC17001) $M$. nodulans (ORS2060) Az. caulinodans (LMG6465)

$R$. rubrum (LMGATCC11170)

A. magnetotacticum (M58171)

Az. brasilense (NCIMB11860)

$A z$. Iipoferum (NCIMB11861)

$R$. acidophila (M34128)

A. Clevelandensis (M69186)

S. morelense (LMG21331) T

$M$. amorphae (LMG18977) T

M. ciceri (LMG14989) T

M. huakuii (LMG14107) T

M. loti (LMG6125) T

M. plurifarium (LMG11892) T

B. elkanii (LMG6134) T

B. beta (LMG21987) T

B. yuanmingense (CCBAU10071) T

S. medicae (A321) T

M. mediterraneum (LMG17148) T

$M$. septentrionale (SDW014) T

$M$. temperatum (SDW018) T

$M$. thiogangeticum (SJT) T $M . t i a n s h a n e n s e(A-1 B S) T$ WC12.1a $L$. sepiaria SA

WC19.1b L. cytisoides SA WC19.1C $L$. cytisoides SA WC23.1b L. multiflora SA

WC23.1c L. multiflora SA

WC23.1d L. multiflora SA

WC28.1F $L$. sericea SA

WC28.1g $L$. sericea SA WC28.1h $L$. sericea SA WC28.1i $L$, sericea SA WC33b L. sericea SA WC33C L. sericea SA ccttgtacac accgcccgtc acaccatggg agttggcttt acccgaagge ccttgtacac accgcccgtc acaccatggg agttggtttt acccgaaggc ccttgtacac accgcccgtc acaccatggg agttggtttt acccgaaggc ccttgtacac accgcccctt ---taagagg agttggtctt -cccgaaggc ccttgtacac accgcccgtc a-agcatggg agttggttt acccgaaggc ccttgtacac accgcccgtc tacccatggg agttggtttt acccgaaggc cettgtacac accgcccgtc aaggcatggg agttggtttt acccgaaggc ccttgtacac accgcccgtc acaccatggg agttggtttt acccgaaggc ccttgtacac accgcccgtc acaccgtggg agttggtttt acccgaaggc ccttgtacac accgcccgtc --cgcatggg agttggtttt acccgaaggc ccttgtacac accgcccgtc a-cccatggg agttggttt acccgaaggc ccttgtacac accgcccgtc acaccatggg agttggtttt acccgaaggc ccttgtacac accgcccgtc acaccatggg agttggttt acccgaaggc ccttgtacac accgcccgtc a-agcatggg agttggtttt acccgaaggc ccttgtacac accgcccgcc --agcatggg agttggtttt acccgaaggc

ccttgtacac accgcccgtc acaccatggg agttggttct acccgaaggt ccttgtacac accgcccgtc acaccatggg agttggttct acccgaaggt ccttgtacac accgcccgtc acaccatggg agttggttct acccgaaggt ccttgtacac accgcccgtc acaccatggg agttggttct acccgaaggt ccttgtacac accgcccgtc acaccatggg agttggttct acccgaaggt ccttgtacac accgcccgtc acaccatggg agttggttct acccgaaggt ccttgtacac accgcccgcc --cccatggg agttggttct acccgaaggt ccttgtacac accgcccgtc acagcatggg agttggttct acccgaaggt ccttgtacac accgcccgtc acaccatggg agttggttct acccgaaggt ccttgtacac accgcccgtc acaccatggg agttggttct acccgaaggt ccttgtacac accgcccgtc --cccatggg agttggttct acccgaaggt ccttgtacac accgcccgtc a-accatggg agttggttct acccgaaggt ccttgtacac accgccc--t gacgcatggg agttggttct acccgaaggt ccttgtacac accgcccgtc -taccatggg agttggttct acccgaaggt

ccttgtacac accgcccgtc acaccatggg agttggttt acctgaagac ccttgtacac accgcccgtc acaccatggg agttggtttt acctgaagac ccttgtacac accgcccgtt a-agcatggg agttggtttt acctgaagac cettgtacac accgcceccc

ccttgtacac accgcccgtc acaacgt-gg agttggtttt acctgaagac ccttgtacac accgcccg-- aacccatggg agttggtttt acctgaagac ccttgtacac accgcccgtc acaccatggg agttggttt acctgaagac ccttgtacac accggccgcc ---ccatggg agttggtttt acctgaagac

cettgtacac accgccegtc acaccatggg agttggtctt acccgacggc ccttctacac accgcccgtc acaccatggg agttggcttt acccgaaggc ccttgtacac accgcccgtc acaccatggg agttggttct acccgaagac ccttgtacac accgccegtc acaccatggg agttggcttt acccgaagcc ccttgtacac accgcccgtc acaccatggg agttggcttt acccgaaggt ccttgtacac accgcccgtc acaccatggg agttggtttt acccgaaggt ccttgtacac accgcccgtc acaccatggg agttggcttt acccgaaggc ccttgtacac accgcccgtc acaccatggg agttggtttt acctgaagac ccttgtacac accgcccgtc acaccatggg agttggttct acccgaaggt ccttgtacac accgcccgtc acaccatggg agttggtttt acccgaaggc

ccttgtacac accgccegtc acaccatggg agttggttt acccgaaggc cettgtacac accgcccgtc acaccatggg agttggtttt acccgaaggc ccttgtacac accgcccgtc acaccatggg agttggttt acccgaaggc ccttgtacac accgcccgtc acaccatggg agttggttt acctgaagac ccttgtacac accgcccgtc acaccatggg agttggttt acctgaaggt ccttgtacac accgcccgtc acaccatggg agttggtttt acctgaagac ccttgtacac accgcccgtc acaccatggg agttggttct acccgaaggt

1460 1470

gctgtgctaa ccg--caagg ag--gca gctgtgctaa ccg--caagg ag--gca gctgtgctaa ccg--caaga gg--gca gctgtgctaa ccg--ca-gt ag--gca gctgtgctaa ccg--caagg ag--gca getgtgctaa $\mathrm{Ccg}-\mathrm{C}-$

gctgtgctaa ccg--caagg ag--gca gctgtgctaa ccg--caagg ag--gca gctgtgctaa ccg--caagg ag--gca gctgtgctaa ccg--caagg ag-gc-gctgtgctaa ccg--caagg ag--gca gctgtgctaa ccg--caagg ag--gca gctgtgctaa ccg--caagg ag--gca gctgtgctaa ccg--caagg ag--gca gctgtgctaa ccg--caagg ag--gca gctgtgctaa ccg--caagg ag--gca gctgtgctaa ccg--caagg ag--gca 
WC33e $L$. sericea SA

WC33h $L$. sericea SA

S. americanum (CFNEI156) T

$S$. arboris (LMG14919) T

S. fredii (LMG6217) T

S. kostiense (LMG19227)

S. kummerowiae (CCBAU71714) T

S. meliloti (LMG6133) T

BH1LSC $L$. spinescens SA

BH2LSa $L$. spinescens SA

BH3LSd $L$. spinescens SA

BW1LSab $L$. spinescens SA

BW2LSa $L$. spinescens SA

BW2LSb $L$. spinescens SA

BW2LSC $L$. spinescens SA

BW3LSd $L$. spinescens SA

$B$. canariense (MSFC2-M3-R-3) T

B. japonicum (LMG6138) T

$B$. liaoningense (LMG18230) T

Betallo6d $L$. pungens SA

Betal106a L. pungens SA

NK22 $L$. sericea SA

WC21.1L $L$. pauciflora SA

XHRla $L$. simsiana SA

Betal106f L. pungens SA

$R$. palustris (ATCC17001)

$M$. nodulans (ORS2060)

Az. caulinodans (LMG6465)

$R$. rubrum (LMGATCC11170)

A. magnetotacticum (M58171)

Az. brasilense (NCIMB11860)

Az. lipoferum (NCIMB11861)

$R$. acidophila (M34128)

A. Clevelandensis (M69186)

S. morelense (LMG21331) T

$M$. amorphae (LMG18977) T

$M$. ciceri (LMG14989) T

M. huakuii (LMG14107) T

M. Loti (LMG6125) T

M. plurifarium (LMG11892) T

B. elkanii (LMG6134) T

B. beta (LMG21987) T

B. yuanmingense (CCBAU10071) T

S. medicae (A321) T getgtgctaa ccg--caagg ag-gc--

agtgcgctaa ccg--caagg ag--gca agtgcgctaa ccg--caagg ag--gca agtgcgctaa ccg--caagg ag--gca agtgcgctaa $\mathrm{ccg}--$ caagg ag--gca agtgcgctaa ccg--caagg ag--gca agtgcgctaa ccg--caagg ag--gca agtgtgctaa ccg--caagg ag--gca agtgtgctaa ccg--caagg ag--gca agtgtgctaa ccg--caagg ag--gca agtgtgctaa ccg--caagg ag--gca agtgtgctaa ccg--caagg ag--gca agtgtgctaa ccg--caagg ag--gca agtgtgctaa ccg--caagg ag--gca agtgtgctaa ccg--caagg ag--gca

ggtgcgctaa ccc--gcaag ggaggca ggtgcgctaa ccc--gcaag ggaggca ggtgcgctaa ccg--aaagg ggcag--

ggtgcgctaa CcC--gcaag ggaggca ggtgcgctaa ccg--aaagg gg--gca ggtgcgctaa cca--gcaat ggaggca ggtgcgctaa ccg--aaagg gg--gca

gctgcgccaa ccg--cgagg gg--gca gttgcgctaa ccg--caagg ag--gca ggtacgctaa ccg--caagg ag--gca ggtgcgctaa ccg--caagg ag--gca ggtgcgctaa ccggcaacgg ag--gca ggtgcgctaa ccg--caagg ag--gca gtttcgctaa ccg--caagg ag--gca ggtgcgctaa ccc--gcaag ga-ggca agtgcgctaa ccg--caagg ag--gca gctgtgctaa ccg--caagg ag--gca

gctgtgctaa ccg--caagg ag--gca gctgtgctaa ccg--caagg ag--gca gctgtgctaa ccg--caagg ag--gca ggtgcgctaa ccg--aaagg gg--gca agtgcgctaa Ccc--gcaag ggaggca ggtgcgctaa ccc--gcaag ggaggca agtgcgctaa ccg--caagg ag--gca 